\title{
Nucleus Pulposus and Mesenchymal Stem Cell Preconditioning: A Focus on Redifferentiation, Wnt Signaling, and Immunity
}

\author{
Tyler Thomas Pizzute
}

Follow this and additional works at: https://researchrepository.wvu.edu/etd

\author{
Recommended Citation \\ Pizzute, Tyler Thomas, "Nucleus Pulposus and Mesenchymal Stem Cell Preconditioning: A Focus on \\ Redifferentiation, Wnt Signaling, and Immunity" (2017). Graduate Theses, Dissertations, and Problem \\ Reports. 6426. \\ https://researchrepository.wvu.edu/etd/6426
}

This Dissertation is protected by copyright and/or related rights. It has been brought to you by the The Research Repository @ WVU with permission from the rights-holder(s). You are free to use this Dissertation in any way that is permitted by the copyright and related rights legislation that applies to your use. For other uses you must obtain permission from the rights-holder(s) directly, unless additional rights are indicated by a Creative Commons license in the record and/ or on the work itself. This Dissertation has been accepted for inclusion in WVU Graduate Theses, Dissertations, and Problem Reports collection by an authorized administrator of The Research Repository @ WVU. For more information, please contact researchrepository@mail.wvu.edu. 


\title{
Nucleus pulposus and mesenchymal stem cell preconditioning: a focus on redifferentiation, wnt signaling, and immunity
}

\author{
Tyler Thomas Pizzute
}

Dissertation submitted to the School of Medicine at West Virginia University in partial fulfillment of the requirements for the degree of

Doctor of Philosophy

In

Exercise Physiology

\author{
Ming Pei, M.D., Ph.D., Chair \\ Stephen E. Alway, Ph.D. \\ Emidio E. Pistilli, Ph.D. \\ Ivan Martinez, Ph.D. \\ Christopher F. Cuff, Ph.D. \\ Division of Exercise Physiology \\ Morgantown, West Virginia \\ 2017
}

Key Words: Nucleus pulposus, intervertebral disc, wnt signaling, ECM, matrix, preconditioning, fibroblast growth factor, stem cell, cartilage

Copyright (C) 2017 Tyler Thomas Pizzute 


\section{ABSTRACT \\ Nucleus pulposus and mesenchymal stem cell preconditioning: a focus on redifferentiation, wnt signaling, and immunity}

Low back pain is one of the most common reasons for doctor visits each year in the United States and consistently represents one of the leading areas of US health care spending in recent decades. Located at the inner space of the intervertebral discs (IVDs) of the spinal column, a soft, shocking absorbing tissue known as nucleus pulposus (NP) is a frequently implicated target of patient symptoms and comorbidities related to back pain. IVDs are quite susceptible to both acute injury, such as disc herniation, and chronic age-related degeneration known as intervertebral disc degeneration (IVDD). These conditions ultimately lead to degeneration and deterioration of the IVD tissue and resident NP cells (NPCs) at the biochemical and cellular level. In recent years, orthopaedic therapies have aspired to utilize cell-based therapies in combination with surgical intervention strategies for the replenishment of NP tissue, however, this approach presents several challenges. Studies which seek to use autologous NPCs (i.e. the patients' own cells) must consider the potential implications and poor efficacy associated with harvesting NPCs from a damaged, typically inflamed environment. Furthermore, initial yield from such procedures is typically limited to the herniated portion of the NP tissue. This avoids compromising the remaining healthy tissue in the disc space. Ultimately, this increases the demand for preconditioning strategies which can effectively: (1) increase cell proliferation and yield, (2) rejuvenate harvested NPCs to a healthy NP phenotype to increase cell redifferentiation/regenerative potential for therapeutic utilization, and (3) accomplishes these goals without causing the preconditioned cells to have any adverse immune responses. In our laboratory, we have previously demonstrated that some of these goals can be accomplished by expanding harvested NPCs on decellularized extracellular matrix (dECM) 
substrates from various sources, however, several key factors remained unexplored. The signaling roles of Wnt (Wingless-Type integration) proteins of the Wnt-signaling pathways in NP redifferentiation (Wnt3A, Wnt5A, and Wnt11) have been previously associated with dECM, however, they have not been fully elucidated or compared; the modulation of these signals could provide more effective and exploitable means for NPC preconditioning. Additionally, it is unknown whether the use of dECM from allogeneic and xenogeneic sources have immunological consequences in the expansion of human NPCs. To demonstrate these concepts, we evaluated several preconditioning strategies for proliferative and redifferentiation effects in multiple cell lines of NPCs and SDSCs in our studies. We also transduced NPCs with lentiviral vectors to overexpress or knockout key WNT genes and evaluated them for redifferentiation gene and protein expression. For dECM studies, we expanded NPCs on allogeneic and xenogeneic dECMs, evaluated NPC redifferentiation, as well as their immunophenotypes by flow cytometry, and assayed T-cell proliferation in a mixed lymphocyte reaction with expanded cells. In these studies, we have shown that it is possible to increase redifferentiation potential of human NPCs and synovial-derived stem cells (SDSCs), a chondrocytic stem cell population, by preconditioning methods including FGF-2 supplementation, dECM expansion, and hypoxic preconditioning. We have demonstrated key roles for Wnt signals in the redifferentiation of NPCs and its relationship with cell preconditioning. Furthermore, we have provided new data and insights surrounding the immunological implications of NPC expansion on allogeneic and xenogeneic-derived dECM substrates. Overall, these findings will allow researchers and clinicians to effectively target Wnt signals, increase redifferentiation capacity, and to understand potential underlying implications of dECM preconditioning for future cell-based orthopaedic therapies. 


\section{ACKNOWLEDGEMENTS}

First, I would like to thank everyone involved in my education and training throughout my academic and professional career. Without the wisdom, patience, and guidance of my teachers, professors, and mentors, none of my achievements would even be possible. I would like to specifically thank my research mentor, Dr. Ming Pei, and my committee members: Dr. Stephen Alway, Dr. Emidio Pistilli, Dr. Ivan Martinez, and Dr. Christopher Cuff for investing their time and efforts to ensure our research studies, and ultimately, my pursuit of a doctorate degree was a success. Dr. Pei works incredibly hard and through his dedication and passion for research, I was lucky to be afforded the opportunity to enter the stem cell field. Dr. Pei is always available to meet with his students and trainees, and ensured that our group's lab efforts were always productive and meaningful. Furthermore, I would like to thank Dr. Kathy Brundage for her time, efforts, and scientific expertise lent to me throughout my graduate career. Also, thank you to Dr. John Hollander for persistent mentorship and giving me a second laboratory home here at WVU. Dr. Hollander and Dr. Alway have helped to ensure that the Department of Exercise Physiology is an exemplary and productive research department, while still looking out for the best interests of students, for which I am greatly appreciative.

Next, I would to thank my colleagues, past and present, at West Virginia University and beyond. I want to thank the doctors and staff in the Department of Orthopaedics, particularly Dr. Sanford Emery and Dr. Brock Lindsey for helping our orthopaedic research department flourish. Thank you to Suzanne Danley, Sheila Rye, and Sherri Leyden for their roles in the department and as my friends and allies, allowing us to conduct our studies. Special thank you to Josh Parenti for keeping the Orthopaedic laboratories running smoothly and for your personal mentorship as a friend and co-worker, and for your assistance in my professional development. 
Thank you to Dr. Xiao-Bing Zhang for your collaborative efforts and expertise. I would like to thank Dr. Jingting Li for her expertise and patience when teaching/supervising me during my early years in the Pei Laboratory. Thank you to Dr. Ying Zhang for your laboratory assistance and collaborations, and to Hollander Lab members: Quincy Hathaway, Danielle Shepherd, Cody Nichols, and Seth Stine for your laboratory assistance and friendships. Thank you to Shani Waris for all of your help inside and outside of the laboratory. Thank you to Karlee and the entire Lobban family for your hospitality and great times in Morgantown. Also, thank you to all summer research students who have assisted us in our efforts over the years. Thank you to Tim, Peggy, and Stuart Squires for your hospitality and the memorable Braxton County adventures. Special thanks go out to Patrick Mitchell, Trevor Groot, Zachary Matchett, Jace Lucas, and Justin Brown for your lifelong friendships, support, and for all the memories we've made together over the years. Last, but certainly not least, thank you to my family, especially my grandmothers, Ruth Congrove and Rebecca Wilson, my parents, Tom and Beverly Pizzute, and my sister, Ashley Pizzute, who have offered me nothing but their unwavering support throughout my life and academic pursuits.

"The harder the conflict, the more glorious the triumph. What we obtain too cheap, we esteem too lightly... I love the man who can smile in trouble, that can gather strength from distress, and grow brave by reflection."

- Thomas Paine, American Crisis (1776) 


\section{DEDICATION}

For My Parents: Tom and Beverly Pizzute

A simple statement cannot completely or accurately express my gratitude for everything that you have done for me. Thank you for being my hard-working, loving, and self-sacrificing parents, who offer me their unconditional love and support in all that I do. Without your encouragement, reassurance, and guidance along this journey, earning my $\mathrm{Ph} . \mathrm{D}$. degree wouldn't have been possible or nearly as meaningful. I'm equally as proud as I am grateful to share in our achievement. You are the most important and influential people in my life, and are truly the foundation for all my successes. I am thankful to have you both as my parents and I love you. 


\section{LIST OF ABBREVIATIONS}

2D

3D

AA

ACAN

ADSC

APO-1

ASC

$\mathrm{AF}$

ALP

APC

ARS

BMP

BMSC

Cas9

CD

CNTL

COL1A1

COL2A1

COLXA1 two-dimensional

three-dimensional

L-ascorbic acid phosphate

Aggrecan

Adipose-derived Stem Cell

apoptosis antigen 1

Adult Stem Cell

Annulus Fibrosis

Alkaline Phosphatase

Allophycocyanin

Alizarin Red S

Bone morphogenetic protein

Bone marrow derived stem cell

CRISPR associated protein 9

Cluster of Differentiation

Control

Collagen Type I

Collagen Type II

Collagen Type X 


\begin{tabular}{|c|c|}
\hline CR & Claret Red \\
\hline CRISPR & Clustered regularly interspaced short palindromic repeats \\
\hline DECM or dECM & Decellularized Extracellular Matrix \\
\hline DNA & Deoxyribonucleic acid \\
\hline ECM & Extracellular Matrix \\
\hline EDHB & Ethyl-3,4-dihydroxybenzoate \\
\hline Fas or FasR & Fas cell surface death receptor \\
\hline FasL & Fas cell surface death receptor ligand \\
\hline FGF & Fibroblast Growth Factor \\
\hline FGF2 or FGF-2 & Fibroblast Growth Factor 2 \\
\hline FGF10 or FGF-10 & Fibroblast Growth Factor 10 \\
\hline FITC & Fluorescein isothiocyanate \\
\hline FOXF1 & Forkhead Box F1 \\
\hline GAG & Glycosaminoglycan \\
\hline GAPDH & Glyceraldehyde-3-Phosphate Dehydrogenase \\
\hline GFP & Green Fluorescent Protein \\
\hline gRNA & Guide Ribonucleic Acid \\
\hline HLA-DR & Human Leukocyte Antigen - antigen D Related (MHC-II) \\
\hline HYPO & Hypoxia \\
\hline $\mathrm{IHC}$ & Immunohistochemistry \\
\hline
\end{tabular}




\begin{tabular}{|c|c|}
\hline IPFP & Infrapatellar fat pad \\
\hline IVD & Intervertebral Disc \\
\hline IVDD & Intervertebral Disc Disease \\
\hline KO & Knockout \\
\hline $\mathrm{kPA}$ & Kilopascal \\
\hline LPL & Lipoprotein lipase \\
\hline MHC & Major Histocompatibility Complex \\
\hline MFI & Median fluorescent intensity \\
\hline $\mathrm{mL}$ & Milliliter \\
\hline $\mathrm{mM}$ & Millimolar \\
\hline mRNA & Messenger Ribonucleic Acid \\
\hline ng & Nanogram \\
\hline $\mathrm{nM}$ & Nanomolar \\
\hline MD & Moderately Degenerated \\
\hline MSC & Mesenchymal Stem Cell \\
\hline NP & Nucleus Pulposus \\
\hline NPC & Nucleus Pulposus Cell \\
\hline $\mathrm{OE}$ & Overexpression/Overexpressing \\
\hline PAX1 & Paired Box 1 \\
\hline PBMC & Peripheral Blood Mononuclear Cell \\
\hline
\end{tabular}




\begin{tabular}{|c|c|}
\hline PBS & Phosphate Buffered Saline \\
\hline PCR & Polymerase Chain Reaction \\
\hline PE & phycoerythrin \\
\hline PI & Proliferation Index \\
\hline PL & Plastic \\
\hline PPAR $\gamma$ & Peroxisome proliferator-activated receptor gamma \\
\hline RUNX2 & Runt-related transcription factor 2 \\
\hline SD & Severely Degenerated \\
\hline SDSC & Synovium-derived Stem Cell \\
\hline siRNA & small interfering ribonucleic acid \\
\hline sgRNA & single guide ribonucleic acid \\
\hline SOX9 & SRY-box 9 \\
\hline SSEA-4 & Stem Cell Embryonic Antigen 4 \\
\hline TGF- $\beta 3$ & transforming growth factor beta3 \\
\hline Wnt or WNT & Wingless-Type integration protein \\
\hline WNT3A & Wingless-Type Integration Site Family, Member 3A \\
\hline WNT5A & Wingless-Type Integration Site Family, Member 5A \\
\hline WNT11 & Wingless-Type Integration Site Family, Member 11 \\
\hline$\mu \mathrm{L}$ & Microliter \\
\hline$\mu \mathrm{M}$ & Micromolar \\
\hline
\end{tabular}




\section{TABLE OF CONTENTS}

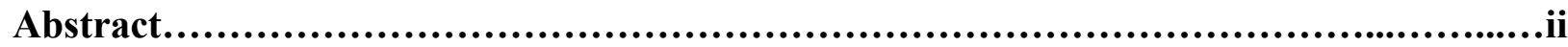

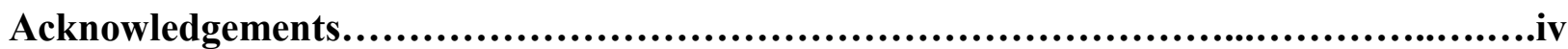

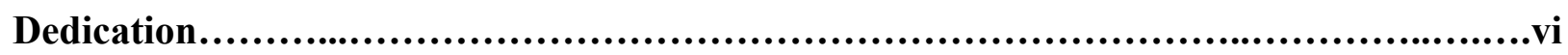

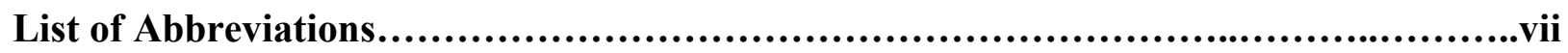

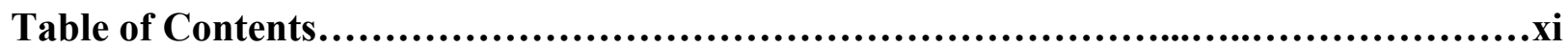

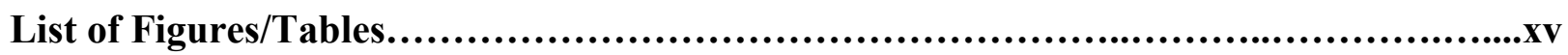

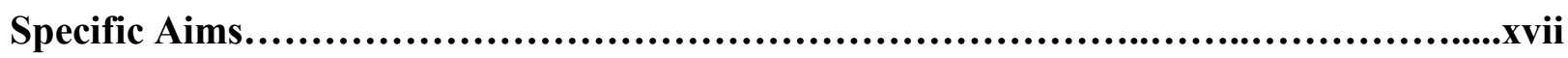

Chapter 1. Literature Review..............................................................1

1.1 Tissue-specific Stem Cells and Lineage-specific Differentiation ..................2

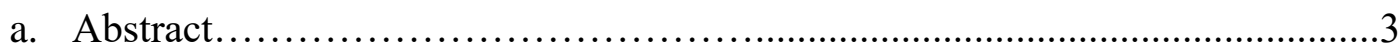

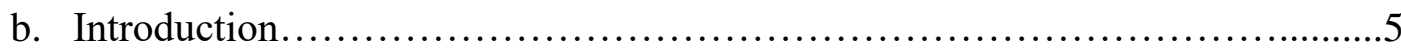
c. Adipogenesis.......................................................
d. Chondrogenesis.................................................9
e. Myogenesis...................................................... 13
f. Osteogenesis........................................................... 16
g. Mechanisms of Lineage Preference......................................20

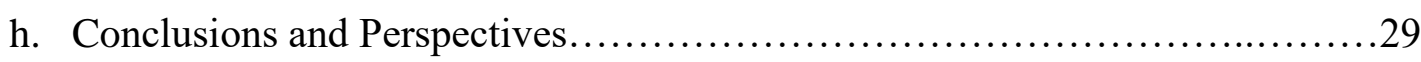

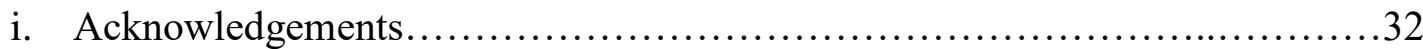
j. References..................................................... 33

1.2 The Intervertebral Disc \& Nucleus Pulposus.....................................50

a. Tissue Morphology................................................. 51

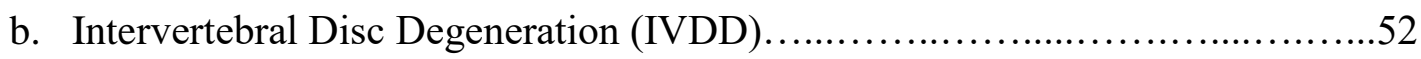

c. IVD and Cell-based Therapeutics........................................55 
d. References.

1.3 Wnt Signaling Pathways.....................................................61

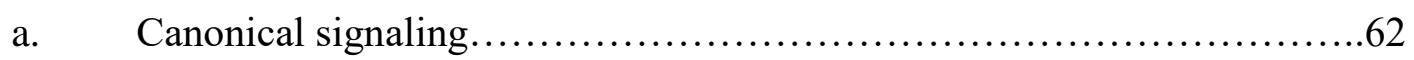

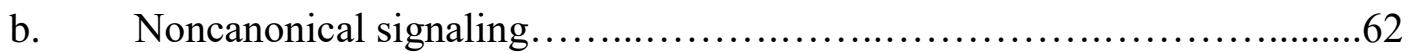

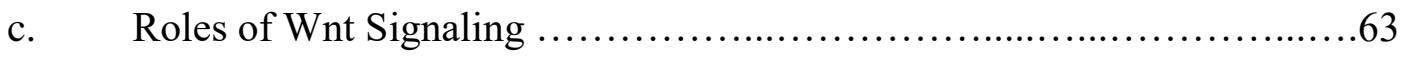

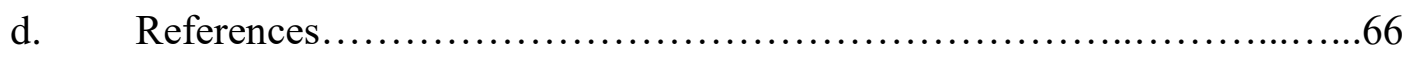

1.4 Preconditioning strategies for nucleus pulposus rejuvenation and impacts on cell

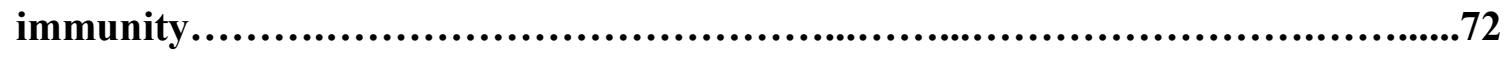
a. $\quad$ Abstract................................................... 73
b. Introduction..................................................... 74
c. FGF-2 Preconditioning ....................................... 76
d. Three-dimensional substrate preconditioning ....................80
e. Detection of immune issues and future directions.................82
f. $\quad$ References................................................... 84

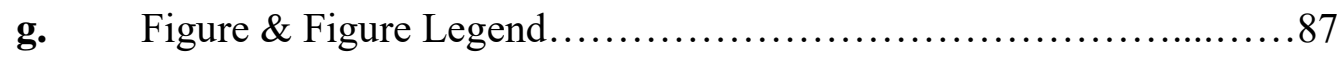

Chapter 2: Fibroblast Growth Factor Ligand Dependent Proliferation and Chondrogenic Differentiation of Synovium-Derived Stem Cells and Concomitant Adaptation of Wnt/Mitogen-Activated Protein Kinase Signals...............................89
a. Abstract. .90
b. Introduction. .91
c. Materials \& Methods. .93
d. Results.
e. Discussion. 102
f. Acknowledgments............................................... 108

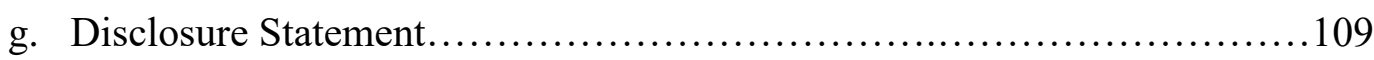
h. References..................................................... 110
i. Figures \& Figures Legends..................................... 117 
Chapter 3: Wnt5A and Wnt11 Signals Impact Redifferentiation in Nucleus Pulposus Cells Harvested from Human Herniated Dis...........................................129
a. Abstract...........................................................

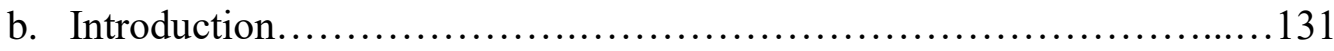
c. Materials \& Methods................................................ 134

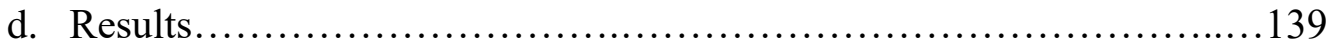

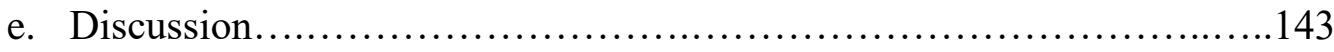
f. Acknowledgements............................................. 148
g. Disclosure Statement................................................. 149

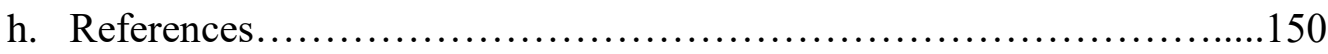
i. Figures \& Figures Legends........................................... 157

Chapter 4: Preconditioning strategies unequally impact regeneration of nucleus pulposus cells from human herniated dises: a proof of principle study..........171

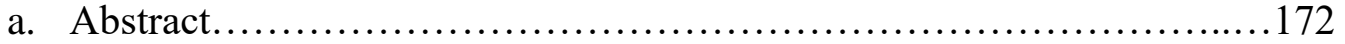

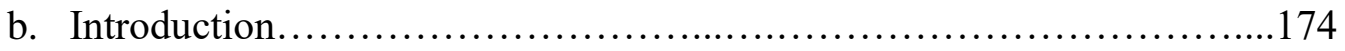

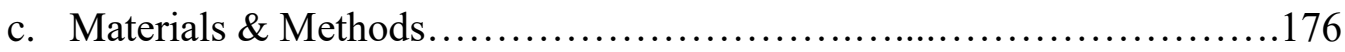

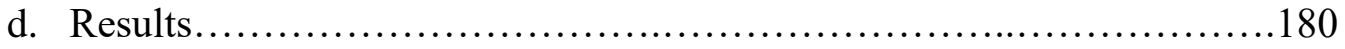

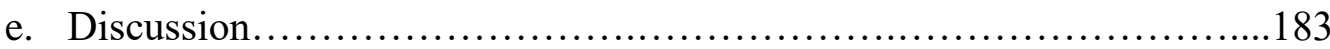
f. Disclosure Statement................................................. 188

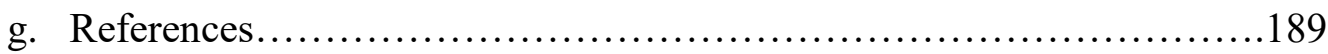
h. Figures \& Figures Legend....................................... 192

Chapter 5: Extracellular matrix preconditioning of nucleus pulposus cells and impacts on immunity ...............................................................204

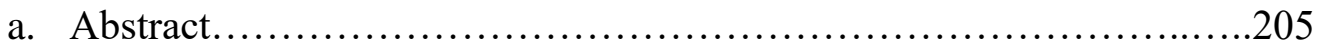

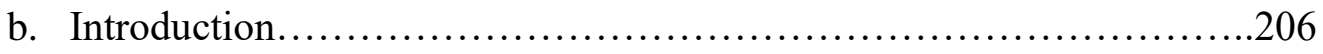

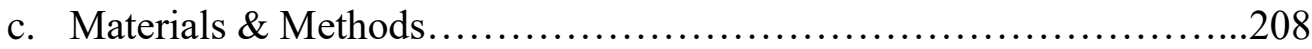

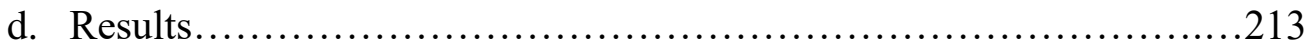




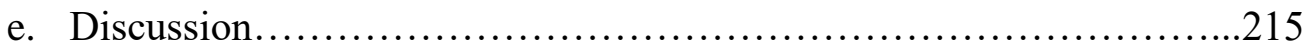

f. Acknowledgements............................................217

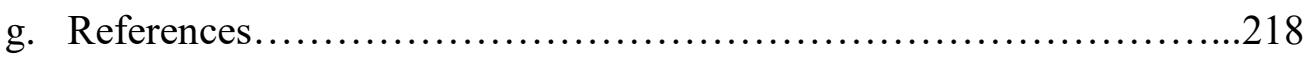

h. Figures \& Figures Legends....................................221

Chapter 6: General Discussion.........................................................231

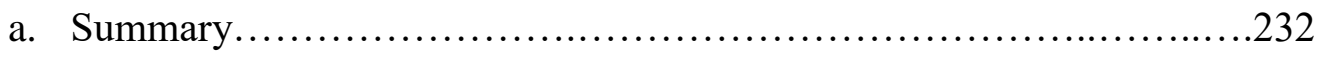
b. Future Directions..............................................233
c. Looking Ahead and Clinical Integration...........................235
d. Figures \& Figure Legends....................................238

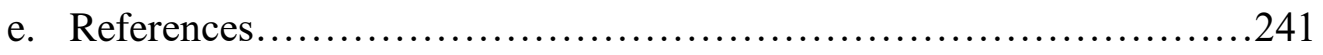

Permissions to Reproduce Copyrighted Materials....................................244

Curriculum Vitae.........................................................................250 


\section{List of Figures/Tables}

\section{Chapter 1.1:}

1.1.1 Adult stem cells can be derived from various tissues in the body.....................46

1.1.2 Epigenetic determination of niche-specific lineage preference.......................48

\section{Chapter 1.2:}

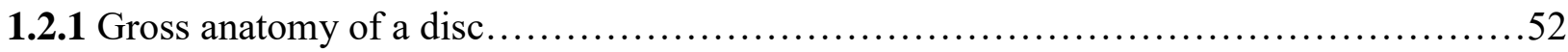

1.2.2 Schematic of the intervertebral disc and anatomic sub-structures......................54

\section{Chapter 1.3:}

1.3.1 Three Wnt-dependent pathways have been categorized.............................70

Chapter 1.4.:

1.4.1 Cell preconditioning and potential immune effects.............................. 87

\section{Chapter 2:}

2.1 Gene expression of FGF ligands............................................ 117

2.2 FGF ligand mediated human SDSC proliferation.................................119

2.3 FGF ligand mediated senescence and differentiation-related gene expression..............121

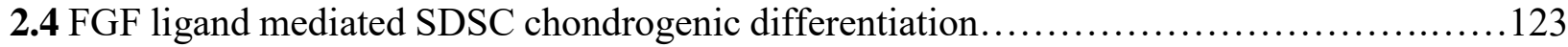

2.5 FGF ligand mediated Wnt and MAPK signal changes in SDSC chondrogenesis............125

2.6 FGF ligand mediated SDSC osteogenic differentiation............................. 127

\section{Chapter 3:}

3.1 Overexpression of WNT genes during redifferentiation............................ 157

3.2 Redifferentiation gene expression in WNT-OE................................ 159 


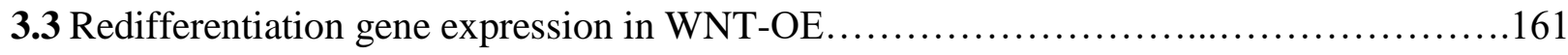

3.4 Redifferentiation of WNT-OE pellets............................................... 163

3.5 Knockout of WNT genes............................................................

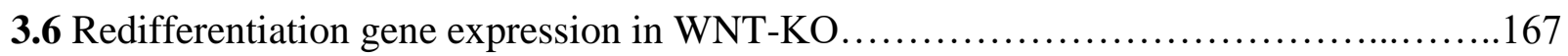

3.7 Redifferentiation of WNT-KO pellets............................................. 169

\section{Chapter 4:}

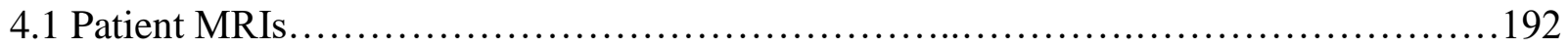

4.2 CD95 and HLA-DR expression of preconditioned NP cells............................194

4.3 Proliferation analysis of expanded NP cells.......................................... 196

4.4 NP cell pellet morphology and redifferentiation induction............................. 198

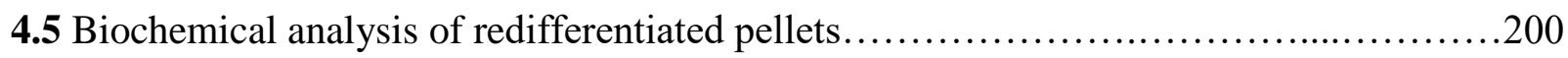

4.6 TaqMan ${ }^{\circledR}$ real-time PCR of redifferentiation pellets..................................202

\section{Chapter 5:}

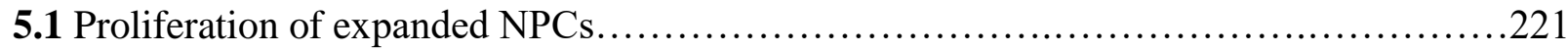

5.2 Immune marker expression following expansion....................................223

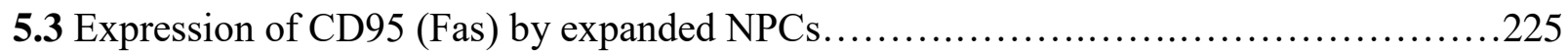

5.4 PBMC proliferation following co-culture with expanded NPCs.......................227

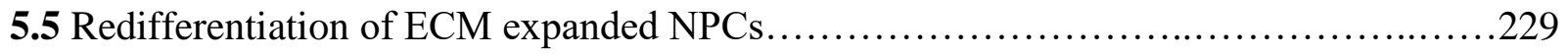

\section{Chapter 6:}

6.1 Immunofluorescence of wildtype (WT) and laminin knockout (KO) NPCs.............238

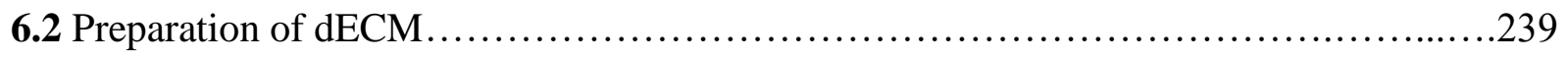

6.3 Biomechanical evaluation of AA treatment study ..................................240 


\section{Specific Aims}

Low back pain (LBP) causes significant morbidity in the United States, is responsible for up to $5 \%$ of healthcare visits $[1,2]$, and affects up to $80 \%$ of the population [3-4]. Additionally, LBP is estimated to cost over $\$ 100$ billion dollars per year in lost wages and costs associated with treatment [1-2]. LBP has been shown to affect a wide population range, including both youth and adolescent individuals [5]. Intervertebral disc disease (IVDD) and degenerative disc disease (DDD) are also significant contributors to this widespread morbidity and financial burden. Both IVDD and DDD are also reliable predictors of future disc complications and injuries directly attributed to these conditions, most notably disc herniation, which is known to cause a significant loss in central NP tissue [2]. Unfortunately, when this loss of NP tissue occurs, it is difficult for NP to naturally heal itself due to its low regenerative capacity, caused by the disc's inherent avascular structure [6] which relies on diffusion to receive nutrient supply. Once injury or degeneration occurs, it can lead to greater disc dysfunction; as the number of healthy NP cells decreases, it leads to further disc dehydration and loss of proteoglycan content, triggering more severe and progressive disc degeneration [2].

The nature of disc degeneration makes autologous cell transplantation an attractive strategy, especially given that autologous cell-based therapy can help to avoid immune rejection and disease transmission; however, a small piece of NP tissue harvested through arthroscopy can only provide a limited number of NPCs. One current challenge in cell transplantation therapy for herniated disc and intervertebral disc disease patients is the lack of healthy implantable cells, which usually cannot be easily harvested in sufficient numbers. Since yield obtained through patient biopsies is 
lower than required, an in vitro expansion method that can produce high quantities of transplantable cells is a reasonable approach. Another challenge surrounding such cell-based therapies for NP repair is the harsh microenvironment of degenerated discs that is generally inhospitable to implanted cells. Overall, a cell expansion method that can yield high quantities of rejuvenated NP cells with preserved redifferentiation capacity would be highly therapeutically relevant for NP cell-based therapies.

\section{Decellularized extracellular matrix (dECM) cell expansion/preconditioning is one proposed}

solution to these challenges, and has been demonstrated in our lab to dramatically enhance cell proliferation, differentiation capacity, and decrease cell senescence in both NP cells and in synovial-derived stem cells [7-8]. By utilizing dECM expansion, cells can be expanded to acquire a large number of cells for future therapeutic purposes. Our recent studies found that dECM deposited by autologous cells can enhance both cell proliferation and differentiation potential of seeded cells and they engage in active Wnt-signaling during expansion and differentiation [7-8]. These findings are consistent with other literature, which have noted roles for canonical and noncanonical Wnt signaling in chondrocytes and cells undergoing chondrogenic differentiation [9$11]$.

Another more common method used for in vitro cell expansion is the supplementation of growth media with fibroblast growth factor 2 (FGF-2). This growth factor has been shown to cause drastic increases in proliferation in a variety of cell types [12-14]. Although it is well known for increasing cell proliferation, its effect on the differentiation capacity following expansion and supplementation in cartilage-like cells [such as synovial-derived stem cells (SDSCs)] is less clear. In a preliminary study performed in our laboratory, we evaluated the ability of FGF-2 and FGF10, another growth factor, to influence SDSC proliferation and differentiation. Interestingly, we 
found that several WNT-family genes were stage-specifically expressed, with WNT3A and WNT5A upregulated and WNT11 downregulated, following several days of FGF-2 treatment during expansion. Later, we discovered this trend in WNT-gene expression also corresponded to successful chondrogenic differentiation, with significant increases in COL2A1 and ACAN mRNA and decreases in COL1A1 expression versus other groups. FGF-10 treatment demonstrated insignificant changes in WNT-gene signaling and consequentially, contrasting trends in mRNA expression as those observed with FGF-2 supplementation during the proliferation stage. Because of the similarities between hyaline cartilage and nucleus pulposus tissue, including their high ratios of collagen type 2, aggrecan, glycosaminoglycan content, and matrix microenvironment [15-17], we hypothesize that Wnt-signaling is also influential on NP redifferentiation. By determining which Wnt signals are most crucial to NP redifferentiation, cell expansion techniques can incorporate various preconditioning strategies, such as drug supplementation or gene therapy, to target the most critical Wnt-signaling pathways to yield the highest quality NP cells for clinical use.

Despite successes with increasing redifferentiation capacity and rejuvenating seeded cells by $\mathrm{dECM}$ expansion, it is unclear whether dECM expansion leads to increased immunogenicity in expanded NP cells. In a recent study from our group, SDSCs expanded on xenogeneic porcine dECM exhibited increases in MHC class II human leukocyte antigen (HLA-DR) expression versus allogeneic dECM and plastic groups [18] which could pose significant challenges for clinicians wishing to use dECM expansion techniques, as increased HLA-DR expression has been shown to be involved in adverse graft rejection events [19-21]. Past studies have demonstrated that MHC class II-positive antigen-presenting cells, and more recently synovial fibroblasts [22] can lead to T-cell activation and immune rejection, although it should be noted that HLA-DR presentation 
alone may not always lead to cell death or T-cell proliferation, but rather T-cell anergy [23-24]. The presence of complementary ligands, CD80 and CD86, B7-1 and B7-2, respectively, must be present in order to activate/proliferate T-cells [25-26] and, since fibroblasts have been shown to engage in HLA-restricted stimulation of T-cells [27], it is important to assure that dECM expansion does not increase the expression of these markers in NP cells. Since chondrocytes, fibroblasts, and synovial stem cells have a similar microenvironment to NP cells, they may experience similar changes in surface marker expression following dECM expansion. By assaying these markers, a more complete surface marker profile can be established for expanded NP cells, as well as new clues and insight into the effects of dECM-expansion on NP cells' immunogenic profiles.

In addition to HLA-DR, CD80, and CD86 expression, other immune markers may be potential concerns for dECM expanded NP cells and their interactions with T-cells. Fas (receptor) and its ligand, FasL, have been studied for many years; their interaction is known to contribute to cell apoptosis, as well as graft rejection and T-cell mediated toxicity [28-29]. By expressing FasL (CD178), cells in immune privileged areas such as the eye [30], testis [30], and intervertebral disc [31] are thought to be able to maintain their survival in the presence of CD95-expressing immune cells. For example, the expression of CD95L has been shown to promote survival of testis cells after implantation in the kidney [30]. It is known that normal, healthy NP cells express FasL [31] and some studies have concluded that NP cells from herniated discs may be susceptible to increased Fas-mediated apoptosis [32] and possibly autoimmunity [33]. By assaying these markers, the protective effects of $\mathrm{dECM}$ expansion can be investigated, as well as additional information regarding NP cell survival in the presence of immune cells.

Injuries and degenerative conditions affecting the intervertebral disc (IVD) and, more specifically, the inner nucleus pulposus (NP) tissue, produces significant morbidity in both young and aging 
patient populations. The nucleus pulposus greatly contributes to the shock absorbing capacity of the IVD under dynamic loading stresses, is relatively avascular, and has a low natural capacity for self-repair following trauma or disease. Chronic degenerative conditions, the natural aging process, and acute injuries such as IVD herniation can produce an inflammatory microenvironment, and consequentially, further contributes to this decreased natural capacity for the native nucleus pulposus to undergo self-repair. Current clinical treatments typically focus on pain management and symptom relief, rather than incorporating cellular strategies to stimulate repair and regeneration of the lost or damaged NP tissue. The primary challenges surrounding such treatments/therapies are the lack of available cells for harvest, as well as the poor cell morphology and low proliferative rates of NP cells obtained through biopsy. To overcome such challenges, the use of decellularized extracellular matrix (dECM) substrates to expand harvested autologous nucleus pulposus cells (NPCs) is an attractive strategy for ex vivo cell expansion; this allows clinicians to harvest a small number of patient cells, which frequently exhibit poor regenerative capacity and, after $\mathrm{dECM}$ expansion, yield a robust number of rejuvenated NP cells for future therapeutic use.

Recent data from our laboratory suggests that the expression of several Wnt-signaling genes coincides with successful redifferentiation of NP cells following dECM expansion and other treatments in vitro. Although $\mathrm{dECM}$ and cell-based tissue engineering strategies have recently begun to provide solutions for biological repair and regeneration of NP tissue, the immunological feasibility surrounding the use of dECM for NP expansion and the specific role of these WNT signals in NP cell redifferentiation have yet to be fully elucidated. We hypothesize that dECM deposited by allogeneic and xenogeneic NP cells can provide superior matrix microenvironments for the rejuvenation of NP cells without significant immune consequences. Additionally, we 
hypothesize that specific WNT-genes (WNT5A, WNT3A, and WNT11) play crucial roles in the redifferentiation of NP cells.

To test our hypotheses, we propose the following specific aims:

[Aim 1.] Determine if the overexpression and/or knockout of WNT3A, WNT5A, and WNT11 in human NPCs can influence their ability to undergo successful NP redifferentiation. The objective of Aim 1 is to test the working hypothesis that Wnt signaling plays a crucial role in NP redifferentiation, with increased canonical Wnt signal (WNT3A) expression leading to less successful NP redifferentiation, and more successful redifferentiation occurring with increased noncanonical Wnt signals (WNT5A and WNT11).

[Aim 2.] Determine if the expansion of human NPCs on plastic, allogeneic dECM, or porcine xenogeneic dECM substrates produces variations in the expression of surface markers related to immune tolerance and increases T-cell proliferation. The objective of Aim 2 is to test the working hypotheses that allogeneic and xenogeneic $\mathrm{dECM}$ expansion are equally appropriate for robust NPC expansion without increasing cell immunogenicity.

The novelty of this proposal includes (1) our investigations of NP regeneration and other closely related cartilage research using dECM rejuvenation; (2) further elucidation of signaling mechanisms related to successful NP redifferentiation and successes using Lentiviral gene knockout and overexpression methods for WNT-genes; (3) preliminary data evaluating the immunological feasibility of using allogeneic and xenogeneic dECM for NPC expansion. Our objective and long-term goal is to define a novel and readily (commercially) available cell expansion system that can provide high quantity, as well as high quality, NP cells for the treatments related to NP regeneration/repair, including patients suffering from chronic degenerative 
conditions or acute disc injury. The primary impact of our expected findings would not only help define crucial roles of WNT-family genes in NP redifferentiation and rejuvenation, but also advance cell-based approaches for NP regeneration and contribute new information regarding the immunological consequences of expanding NP cells on decellularized matrix microenvironments. 


\section{References}

[1] Schwarzer A.C., Aprill C.N., Derby R., et al. The relative contributions of the disc and zygapophysealjoint in chronic low back pain. Spine 1994; 19:801-806.

[2] Kepler CK, Ponnappan RK, Tannoury CA, Risbud MV, Anderson DG. The molecular basis of intervertebral disc degeneration. Spine 2013; 13; 3:318-30.

[3] Luo X, Pietrobon R, Sun SX, Liu GG, Hey L Estimates and patterns of direct health care expenditures among individuals with back pain in the United States. Spine (Phila Pa 1976) 2004; 29(1):79-86.

[4] Borenstein D. Epidemiology, etiology, diagnostic evaluation, and treatment of low back pain. Curr Opin Rheumatol. 1992; 4(2):226-32.

[5] Phélip X. Why the back of the child? Eur Spine J 1999; 8:426-8.

[6] Sakai D, Andersson GBJ. Stem cell therapy for intervertebral disc regeneration: obstacles and solutions. Nature Reviews Rheumatology 2015; 11, 243-256

[7] Zhang Y, Li J, Davis ME, Pei M. Delineation of in vitro chondrogenesis of human synovial stem cells following preconditioning using decellularized matrix. Acta Biomater. 2015; 20, 39-50.

[8] Li J, Hansen KC, Zhang Y, Dong C, Dinu CZ, Dzieciatkowska M, Pei M. Rejuvenation of chondrogenic potential in a young stem cell microenvironment. Biomaterials. 2014; 35(2):642-53. 
[9] Sassi N, Laadhar L, Allouche M, Zandieh-Doulabi B, Hamdoun M, Klein-Nulend J, Makni S, Sellami S. The roles of canonical and non-canonical Wnt signaling in human de-differentiated articular chondrocytes. Biotech Histochem. 2014; (1):53-65.

[10] Lu C, Wan Y, Cao J, Zhu X, Yu J, Zhou R, Yao Y, Zhang L, Zhao H, Li H, Zhao J, He L, Ma G, Yang X, Yao Z, Guo X. Wnt-mediated reciprocal regulation between cartilage and bone development during endochondral ossification. Bone. 2013 (2):566-74.

[11] Bradley EW, Drissi MH. WNT5A regulates chondrocyte differentiation through differential use of the CaN/NFAT and IKK/NF-kappaB pathways. Mol Endocrinol. 2010; 24:1581-93.

[12] Naruse M, Shibasaki K, Ishizaki Y. FGF-2 signal promotes proliferation of cerebellar progenitor cells and their oligodendrocytic differentiation at early postnatal stage. Biochem Biophys Res Commun. 2015; 463(4):1091-6.

[13] An S, Huang X, Gao Y, Ling J, Huang Y, Xiao Y. Int J Mol Med. FGF-2 induces the proliferation of human periodontal ligament cells and modulates their osteoblastic phenotype by affecting Runx2 expression in the presence and absence of osteogenic inducers. 2015; 10, 705711.

[14] Nagayasu-Tanaka T, Anzai J, Takaki S, Shiraishi N, Terashima A, Asano T, Nozaki T, Kitamura M,Murakami S. Action Mechanism of Fibroblast Growth Factor-2 (FGF-2) in the Promotion of Periodontal Regeneration in Beagle Dogs. PLoS One. 2015; 10(6) 1091-6

[15] Sivan, S.S. Hayes, A.J. Wachtel, E. Caterson, B. Merkher, Y. Maroudas, A. Brown, S. Roberts, S. Biochemical composition and turnover of the extracellular matrix of the normal and degenerate intervertebral disc. Eur. Spine J. 2014; 23, 344-353. 
[16] Lotz, M.; Loeser, R.F. Effects of aging on articular cartilage homeostasis. Bone 2012, 51, $241-248$.

[17] Rosenzweig DH, Carelli E, Steffen T, Jarzem P, Haglund L.. 3D-Printed ABS and PLA Scaffolds for Cartilage and Nucleus Pulposus Tissue Regeneration. Int J Mol Sci. 2015; 16(7):15118-35.

[18] Zhang Y, Pizzute T, Li J, He F, Pei M. Biomaterials. 2015 64:88-97. sb203580 preconditioning recharges matrix-expanded human adult stem cells for chondrogenesis in an inflammatory environment - A feasible approach for autologous stem cell based osteoarthritic cartilage repair.

[19] Claesson K. Scand. Mechanisms in organ allograft rejection. An experimental and clinical study. J Urol Nephrol Suppl. 1987; 103:1-42.

[20] Auböck J, Irschick E, Romani N, Kompatscher P, Höpfl R, Herold M, Schuler G, Bauer M, Huber C, Fritsch P. Rejection, after a slightly prolonged survival time, of Langerhans cell-free allogeneic cultured epidermis used for wound coverage in humans. Transplantation. 1988 45(4):730-7.

[21] Döring M, Rohrer KM, Erbacher A, Gieseke F, Schwarze CP, Bader P, Handgretinger R, Hofbeck M, Kerst G. Human leukocyte antigen DR surface expression on CD14+ monocytes during adverse events after hematopoietic stem cell transplantation. Hematol. 2015; 94(2):265-73.

[22] Kraft M, Filsinger F, L. Kramer KL,* Kabelitz D, Hansch G. Schoels MM. Synovial fibroblasts as accessory cells for staphylococcal enterotoxin-mediated T-cell activation. Immunology 1995; (85)461-466. 
[23] Kudo H, Matsuoka T, Mitsuya H, Nishimura Y, Matsushita S. Cross-linking HLA-DR molecules on Th1 cells induces anergy in association with increased level of cyclin-dependent kinase inhibitor p27 (Kip1). Immunol Lett. 2002; 81(2):149-55.

[24] Corrigall VM, Solau-Gervais E, Panayi GS. Lack of CD80 expression by fibroblast-like synoviocytes leading to anergy in T lymphocytes. Arthritis Rheum. 2000; 43(7):1606-15.

[25] Lafferty KJ, Cunningham AJA. New analysis of allogeneic interactions: Lafferty and Cunningham extended the Bretscher and Cohn two-signal model to suggest that $\mathrm{T}$ cells require two signals for activation. J. Immunol. 1974; 112:436-437.

[26] Chen L, Flies DB. Molecular mechanisms of T cell co-stimulation and co-inhibition. Nat Rev Immunol.2013; 13(4):227-242.

[27] Smythe JA, Fink PD, Logan GJ, Lees J, Rowe PB, Alexander IE. Human fibroblasts transduced with CD80 or CD86 efficiently trans-costimulate CD4+ and CD8+ T lymphocytes in HLA-restricted reactions: implications for immune augmentation cancer therapy and autoimmunity. J Immunol. 1999; 163(6):3239-49.

[28] Bellgrau D, Gold D, Selawry H, Moore J, Franzusoff A, Duke RC. A role for CD95 ligand in preventing graft rejection. Nature. 1995; 377(6550):630-2.

[29] Suda T, Takahashi T, Golstein P, Nagata S. Molecular cloning and expression of the Fas ligand, a novel member of the tumor necrosis factor family. Cell. 1993; 75(6):1169-78.

[30] Griffith TS, Brunner T, Fletcher SM, Green DR, Ferguson TA. Fas ligand-induced apoptosis as a mechanism of immune privilege. Science. 1995; 270(5239):1189-92. 
[31] Takada T, Nishida K, Doita M, Kurosaka M. Fas ligand exists on intervertebral disc cells: a potential molecular mechanism for immune privilege of the disc. Spine (Phila Pa 1976) 2002; 27:1526-1530.

[32] Park JB, Kim KW, Han CW, Chang H. Expression of Fas receptor on disc cells in herniated lumbar disc tissue. Spine (Phila Pa 1976). 2001; 26(2):142-6.

[33] Wang J, Tang T, Yang H, Yao X, Chen L, Liu W, Li T. The expression of Fas ligand on normal and stabbed-disc cells in a rabbit model of intervertebral disc degeneration: a possible pathogenesis J Neurosurg Spine. 2007; (5):425-30. 
Chapter 1:

\section{Literature Review}




\section{Chapter 1.1}

\section{Impact of tissue-specific stem cells on lineage-specific}

\section{differentiation: a focus on the musculoskeletal system.}

As published in Stem Cell Rev.; Feb. 2015

Tyler T. Pizzute, Kevin Lynch, Ming Pei.

Stem Cell and Tissue Engineering Laboratory, Department of Orthopaedics, West Virginia

University, One Medical Center Drive, PO Box 9196, Morgantown, WV 26506-9196, USA.

Running Title: tissue-specific stem cells

Corresponding author: Ming Pei, $\mathrm{MD}, \mathrm{PhD}$

Stem Cell and Tissue Engineering Laboratory, Department of Orthopaedics, West Virginia University, PO Box 9196, One Medical Center Drive, Morgantown, WV 26506-9196, USA, Telephone: 304-293-1072; Fax: 304-293-7070; Email: mpei@ hsc.wvu.edu 


\begin{abstract}
Tissue-specific stem cells are found throughout the body and, with proper intervention and environmental cues, these stem cells exercise their capabilities for differentiation into several lineages to form cartilage, bone, muscle, and adipose tissue in vitro and in vivo. Interestingly, it has been widely demonstrated that they do not differentiate with the same efficacy during lineage-specific differentiation studies, as the tissue-specific stem cells are generally more effective when differentiating toward the tissues from which they were derived. This review focuses on four mesodermal lineages for tissue-specific stem cell differentiation: adipogenesis, chondrogenesis, myogenesis, and osteogenesis. It is intended to give insight into current multilineage differentiation and comparative research, highlight and contrast known trends regarding differentiation, and introduce supporting evidence which demonstrates particular tissue-specific stem cells' superiority in lineage-specific differentiation, along with their resident tissue origins and natural roles. In addition, some epigenetic and transcriptomic differences between stem cells then may explain the observed trends are discussed.
\end{abstract}


Keywords: Tissue-specific stem cell, Osteogenesis, Adipogenesis, Chondrogenesis, Myogenesis, Epigenetics, Transcriptomics 


\section{1a Introduction}

Humans and other higher eukaryotes utilize various populations of stem cells throughout the developmental process and into adulthood. The vast repertoire of functional stem cell populations is imperative to normal cellular and tissue renewal. Despite possessing a high degree of pluripotency and proliferative potential (1), embryonic stem cell research has been met with various ethical concerns and strict regulations, especially in the United States, restricting the use of such stem cells in research and clinical settings. These obstructions have forced scientists to search for alternative approaches in stem cell therapy, shifting research focus to the utilization of somatic stem cells for regenerative medicine and tissue engineering.

Somatic stem cells, commonly referred to as adult stem cells (ASCs) or tissue-specific stem cells, are present throughout various tissues in the body (2). Tissue-specific stem cells are multipotent and self-renewing cells which possess endogenous functions for tissue renewal and repair at their respective resident tissues (3). Although ASCs seem to exist ubiquitously throughout a variety of tissues, current literature suggests that not all are necessarily created equal in their differential and proliferative capacities, or their ability to respond to outside influences such as microenvironments. In reality, ASCs have inherent properties which greatly contribute to their ability to undergo successful single lineage-specific differentiation. Great variability in the differential capacity certainly exists between tissue-specific stem cells, which may vary within the same cell type. Populations and subpopulations of cells derived from the same tissue may exhibit slight variations in surface marker expression or in their expression of a single gene, which may alter their tendency to engage in uniform lineage-specific differentiation. For researchers investigating stem cell-based tissue engineering, it is necessary to choose the most appropriate type of ASCs naturally suited to the research goals and objectives. Many times, 
the inherent properties of tissue-specific stem cells are overlooked. This review focuses on four mesodermal lineages for ASC differentiation: adipogenesis, chondrogenesis, myogenesis, and osteogenesis. It is intended to review the current multilineage differentiation and comparative research, highlight and contrast known trends regarding differentiation, and introduce supporting evidence which demonstrates particular ASCs' superiority in lineage-specific differentiation, concomitant with their resident tissue origins and natural roles. In addition, some epigenetic and transcriptomic differences between stem cells which may explain the observed trends are discussed. 
Tissue-Specific Stem Cells Benefiting Lineage-Specific Differentiation

\section{1b Adipogenesis}

Although commonly removed via liposuction surgery, newly differentiated ASCs from adipose tissue have therapeutic potential in cosmetic surgery (2), as well as tissue grafts for burn victims and autologous transplantation (4). The use of adipose-derived stem cells (ADSCs) in lineage-directed studies has been established, with their greatest success demonstrated along the adipogenic lineage (Figure 1). In a multilineage comparison study by Yoshimura and colleagues using murine ASCs, the greatest adipogenic potential was observed using Oil-Red-O staining in the groups from both synovial-derived stem cells (SDSCs) and ADSCs compared to those from muscle-derived stem cells (MDSCs), periosteum-derived stem cells, and bone marrow-derived stem cells (BMSCs). These findings were supported by reverse transcription polymerase chain reaction (RT-PCR) results for adipogenic markers [peroxisome proliferator-activated receptor gamma (PPARG) and CCAAT/enhancer binding protein alpha (CEBPA)] after four days of adipogenic lineage induction (5). These conclusions were consistent with the findings by Sakaguchi and colleagues. They found that the SDSC and ADSC groups represented the only groups with each of their three colonies stained positive for lipid accumulation; the BMSC group had one colony with a staining rate greater than $80 \%$. In contrast, the periosteum and MDSC groups had zero colonies possessing a rate of Oil-Red-O staining greater than $80 \%$, which is indicative of being highly inferior for adipogenesis (6). These results are further supported by the work of Mochizuki and colleagues, where differences between SDSCs harvested from fibrous synovium, SDSCs from adipose synovium, and subcutaneous ADSCs were indistinguishable in Oil-Red-O staining (7). In a multilineage study by Peng and colleagues, rat ADSCs exhibited the greatest normalized PPARG and lipoprotein lipase (LPL) levels at day 7 in an adipogenic 
induction regimen, demonstrating superior adipogenic potential of ADSCs to BMSC and cartilage-derived stem cell groups, which was further confirmed by densitometric analysis of Oil-Red-O stained cultures (8). Based on these studies, it appears that SDSCs and ADSCs can each undergo successful adipogenic differentiation. More studies need to be conducted in order to determine if definitive adipogenic superiority exists between the two cell types.

In another comparative study using several types of tissue-specific stem cells, ADSCs were directly compared with BMSCs after seeding on collagen scaffolds. Despite similar trilineage differentiation overall (chondrogenic, adipogenic, and osteogenic) between both groups, there was a significantly greater and more rapid upregulation of adipogenic genes in ADSCs and osteogenic genes in BMSCs after in vitro induction (9). A transcriptomics study by Monaco and colleagues aimed to compare the differentially expressed genes of ADSCs derived from adult porcine subcutaneous adipose tissue and BMSCs derived from the femur before and after osteogenic and adipogenic differentiation (10). Just as Vishnubalaji and colleagues observed (11), Monaco and colleagues found that ADSCs had greater lipid metabolism than BMSCs while BMSCs had an increased osteogenic and proliferative capacity; ADSCs exhibited significantly lower expression for osteopontin (OPN) than BMSCs, which was also confirmed by quantitative RT-PCR. Based upon their functional analyses, it is reasonable to suggest that ADSCs naturally progress toward the adipogenic lineage with greater propensity than BMSCs and vice versa (10). 


\section{1c Chondrogenesis}

Producing healthy, viable human cartilage for surgical repair through autologous transplantation has widespread therapeutic potential, especially for patients in the aging populations. The synovium has proved to be a valuable source of ASCs for effective induction of chondrogenesis and the production of high-quality cartilage in vitro $(12,13)$ and in vivo $(14)$, but it has also been investigated in osteogenic, adipogenic, and myogenic experiments (Figure 1).

SDSCs have a tendency to progress toward the chondrogenic lineage more effectively than other stem cells. Mochizuki and colleagues found that human SDSCs from both fibrous and adipose synovium exhibited similar superiority over subcutaneous ADSCs in chondrogenic potential (7). Another study comparing various human ASCs from separate sources was performed by Sakaguchi and colleagues, where SDSCs were once again the most superior source for stem cell chondrogenesis over ADSCs and MDSCs; the SDSC group yielded pellets with the largest size and the highest intensity for toluidine blue cartilage matrix staining (6). Similar conclusions were supported by Yoshimura and colleagues, who reported that rat SDSCs exhibited the greatest efficiency and growth kinetics, producing the heaviest chondrogenic pellets due to matrix formation (5). Compared to BMSCs, ADSCs exhibited a reduced chondrogenic potential under standard culture conditions driven by transforming growth factor beta (TGF $\beta$ ). Hennig and colleagues found that human ADSCs had reduced expression of bone morphogenetic protein-2 (BMP2), -4 (BMP4), and -6 (BMP6) mRNA and did not express TGF $\beta$ receptor-1 protein. BMP6 treatment induced TGF $\beta$-receptor-1 expression and combined application of TGF $\beta$ and BMP6 eliminated the reduced chondrogenic potential of ADSCs inducing a gene expression profile similar to differentiated BMSCs. Similar to BMSCs, chondrogenesis of ADSCs was associated with hypertrophy according to premature collagen X 
(COL10A1) expression, upregulation of ALP activity, and in vivo calcification of spheroids after ectopic transplantation in SCID mice (15). Although this study did not use SDSCs (in addition to BMSCs and ADSCs) to similarly compare their hypertrophy or calcification fates, SDSCs have been evaluated in other studies. In a report using an osteogenic induction medium, SDSCs exhibit a 5- to 10-fold decrease compared to BMSCs in the levels of osteocalcin (OCN) and ALP (16), which are known to contribute to calcification and pro-osteoblast activity; however, the generation of articular cartilage without hypertrophic terminal differentiation still remains a current challenge in the field (17).

Several studies have compared the in vivo efficacy and capabilities of SDSCs for cartilage regeneration and repair of osteochondral defects in rabbit models. After initially demonstrating that SDSCs were superior stem cells for chondrogenesis, Koga and colleagues transplanted donor-matched ASCs to repair cartilage defects created in a rabbit model and found that SDSCs and BMSCs produced significantly greater amounts of cartilage matrix than other cells of adipose and muscle tissue origins; when SDSCs were transplanted at a higher cell density and with a periosteal patch, more abundant cartilage matrix was observed. They also noted that SDSCs had a clear advantage in terms of proliferative potential, giving SDSCs an additional edge over BMSC counterparts for therapeutic applications (18). In another similar in vivo experiment, Pei and colleagues set out to repair full-thickness rabbit cartilage defects via allogeneic in vitro engineered SDSC cartilage constructs. Six months after implantation of SDSC-based constructs, the femoral condyle defects were filled with smooth hyaline-like cartilage, did not exhibit collagen I, and possessed high levels of collagen II and glycosaminoglycan (GAG), with well integrated new tissue formation. These results are contrasted by control groups which possessed fibrous tissue (14). A third study using a rabbit 
model for defective articular cartilage repair, Lee and colleagues also tested SDSCs' in vivo effectiveness. For this study, SDSCs were seeded in a platelet-rich plasma (PRP) gel, which could be injected into the femoral defect. After 24 weeks, results very similar to the Pei et al. study were obtained, with fibrous tissue in the control group and hyaline cartilage in both the PRP group and the PRP-SDSC group. The PRP with seeded SDSCs possessed greater GAG content than the non-SDSC groups, as well as the greatest collagen II expression (19). In another relevant study, hydrogel encapsulated porcine SDSCs, BMSCs, and ADSCs were compared for in vitro and in vivo chondrogenesis, SDSCs were once again found to be the most chondrogenic. SDSCs yielded mechanically stiffer constructs and as others have found, SDSC hydrogels exhibited the greatest GAG and collagen expression of any group (20).

SDSCs' success in chondrogenesis seems to lie in their inherent cellular properties and growth characteristics $(17,21)$. One study found that chondrocytes and intraarticular tissue stem cells (including SDSCs) from human donors exhibited a higher expression of proline argininerich end leucine-rich repeat protein (PRELP), a connective tissue glycoprotein of the leucine-rich repeat family abundant in cartilage rather than in cultured fibroblasts, which was absent in extraarticular tissue stem cells, such as ADSCs and MDSCs; BMSCs increased PRELP expression during in vitro chondrogenesis (22). After many passages, ASCs tend to undergo a process marked by telomere shortening and replicative senescence, leading to impaired ability to differentiate into specific tissues (23). SDSCs retain multipotency for up to ten passages with limited cell senescence and retained chondrogenic capacity (24). This characteristic presents a reasonable explanation for SDSCs' remarkable ability to successfully differentiate into cartilaginous tissue and, to a lesser degree, yet notably, the ability to produce muscle, bone, and adipose tissue. It is also notable that the in vitro microenvironment can influence SDSC 
differentiation toward chondrogenesis, particularly the extracellular matrix (ECM). ECM deposited by SDSCs has been shown to improve SDSC expansion in vitro and shift the SDSCs at a greater propensity toward the chondrogenic lineage, while decreasing osteogenesis and adipogenesis $(25,26)$. 


\section{1d Myogenesis}

Several potential therapeutic applications for myogenically differentiated stem cells exist, including dystrophic diseases and orthopaedic surgery (27). Therapies which produce viable muscle tissue have the potential to aid against the pathogenicity of muscle diseases and elucidate natural mechanisms for muscle repair via ASCs. The contribution of MDSCs to myogenic differentiation in vitro have been investigated, as well as their ability to contribute to muscle tissue in vivo. Following a similar trend as other tissue-specific stem cells, MDSCs seem to most effectively undergo myogenesis than other types of lineage specification (Figure 1).

Several muscle progenitor populations have been identified in muscle which do not express satellite cell markers such as Pax7, and some of these populations have been shown to be myogenic in vivo and in vitro. One such population located in the interstitium of postnatal muscle, expressing PW1, a cell stress mediator, is referred to as PW1+/Pax7- interstitial cells (PICs). Mitchell and colleagues found that PICs exhibited comparable levels of myogenesis to that of satellite cells in vivo and engaged in the stem cell process of self-renewal. Interestingly, PICs require Pax7 for myogenic specification, as none of the Pax7-deficient PICs was deemed myogenic (28). Differing from the commonly researched and highly myogenic populations of Pax7+ muscle satellite cells $(27,29)$, other MDSCs can be multipotent and have the capacity to differentiate into many cell types such as myocytes, chondrocytes, adipocytes, and osteocytes under the necessary conditions (30). Aside from PICs, other muscle-derived cell populations have been discovered and evaluated for their contributions to muscle repair (31-34). Some of these muscle-derived side populations may be able to form new myotubes and contribute to muscle repair and regeneration (35). It should be noted that, despite the fact that several populations expressing mesenchymal stem cell markers have been identified and can engage in 
multilineage differentiation, their stem cell status is currently debated in the field; however, it is accepted that non-satellite muscle cells accumulate in the interstitium following muscle injury and can contribute to muscle repair in the presence of necessary environmental or outside factors (31), leading some investigators to speculate about their roles in the repair of damaged muscle.

In a study by Meligy and colleagues, ADSCs, BMSCs, and skeletal MDSCs were harvested from six-week-old rats for in vitro myogenic comparative studies. Flow cytometry data showed that all stem cells exhibited positive expression of CD90 and CD44 and lacked expression of CD35, CD41, and CD34. Under myogenic induction, the greatest myogenic marker expression was exhibited by the skeletal MDSC population with peak myogenin expression of $93 \%$ in the myogenically differentiated MDSCs, $83.3 \%$ in the BMSCs, and $77 \%$ in the ADSCs (36). The similarity of myogenic potential between BMSCs and ADSCs was also demonstrated in another report using rats. After four passages, investigators observed high expression of CD90 in both ADSCs and BMSCs and a reduction of CD44 expression in ADSCs. They also observed significantly higher expression of myogenic differentiation 1 (MyoD1) in BMSCs compared to ADSCs (37). In a related comparative study conducted by Lei and colleagues, after a 28-day myogenic induction, higher expression levels of skeletal musclespecific genes were observed in adult mouse MDSCs than fetal counterparts $(\mathrm{p}<0.01)$ and the lowest expression levels were demonstrated in ADSCs $(\mathrm{p}<0.01)$. All stem cells were detected for both CD29 and CD90 positive and CD45 negative phenotype, and exhibited fibroblast-like spindle morphology in cell cultures. In addition, muscle-specific cadherin (M-Cad) and myosin heavy chain (MyHC) expressions in ADSCs were not detected by immunofluorescence or quantitative real-time PCR (38). These results suggest that some inherent properties may exist in non-satellite MDSC populations, which allowing the MDSC populations to more readily 
upregulate myogenic genes and progress along the myogenic lineage than stem cells from alternative sources.

Satellite cells and other MDSC side populations seem to be the most natural choice for producing quality myotubes; however, other stem cells have been utilized, despite the fact that ADSCs appear to be a poor choice. In two separate experiments by De Bari and colleagues, SDSCs were evaluated for myofiber incorporation and myogenic capacity $(24,39)$. In the earlier study from 2001, five SDSC clones were evaluated for adipogenic, myogenic, chondrogenic, osteogenic, and myogenic differentiation capacity. All clones were determined to be fully capable of chondrogenesis, adipogenesis, and osteogenesis; however, this myogenic differentiation was described as a "few scattered, rudimentary myotubes" (24). In their later 2003 study, using the in vivo mdx mouse model and tibialis anterior muscle injection of human SDSCs, they found that SDSCs possessed the capacity to contribute to myofiber formation, independent of fusion with muscle cells. Successful myogenesis occurred and the implanted SDSCs were able to contribute to the local satellite cell population (39). More research is needed to truly elucidate the complete differential capabilities of non-satellite muscle-derived cell populations, as well as an accurate method for classifying the status of these populations as stem cells. 


\section{1e Osteogenesis}

In conjunction with cartilage engineering studies, experiments which aim to produce bone tissue are crucial and invaluable to the medical community. Bone constructs produced from stem cells can be used in fracture repair, as well as treating bone tissue defects (40). Although BMSCs have been evaluated for multilineage potential, especially chondrogenesis, they most effectively undergo osteogenic differentiation (Figure 1).

Several in vitro studies have been performed which demonstrate the superior capabilities of BMSCs to differentiate into bone tissue. A comparison study by Im and colleagues set out to determine the difference between the chondrogenic and osteogenic capacity of ADSCs and BMSCs by differentiating these cells on a monolayer culture. Based on the results of Von Kossa matrix mineralization assay and alkaline phosphatase (ALP) staining for osteoblastic differentiation, the BMSCs proved to be superior to the ADSCs (41). This conclusion was also in agreement with another comparative study by Vishnubalaji and colleagues (11). In a study which took a less common approach to evaluate osteogenesis, Park and colleagues used Chip-Based assays to measure osteogenic markers and gene expression to compare the potential of human BMSCs and ADSCs for bone formation. Using hydraulic pressure to add cell stress, they saw increases in bone matrix formation in both cell types; however, stimulated BMSCs showed greater staining in Alizarin Red S and ALP assays which is indicative of osteogenesis. They concluded that BMSCs were more susceptible to changes in osteogenic differentiation under mechanical stimulation than ADSCs (42). This conclusion seems reasonable when one considers the weight bearing responsibility and mechanical stability demand of the human skeletal system, which is likely a manifestation of the susceptibility and responsiveness of osteoblastic precursor and BMSC populations to such mechanical forces. 
These growth and differentiation characteristics may also contribute to their natural and specific inclination toward the osteogenic lineage, as well as their role as effective ASCs for bone growth and formation. This idea was tested by Muraglia and colleagues with BMSCs for osteogenesis, chondrogenesis, and adipogenesis after producing non-immortalized clones. In two clone groups, $60 \%$ and $80 \%$ of clones in each respective group were bipotent toward the osteochondrogenic lineage. They found that some groups of BMSC clones do in fact possess trilineage potential at the clonal level; however, the BMSCs studied seem to favor the osteogenic lineage, as they shed their multipotency and all clones progressed toward osteogenic differentiation. All clones exhibited this osteogenic bias. Notably, certain clonal phenotypes were not observed in the study, such as clones which expressed the chondrogenic or adipogenic phenotypes exclusively (43).

Other support for BMSCs' superiority in osteogenesis is highlighted in a report that, compared with ADSCs and SDSCs, equine BMSCs exhibited significant five-fold increases in runt-related transcription factor 2 (RUNX2) levels on day 7 of osteogenic differentiation and a six-fold increase in expression by day 14; levels of osteoblast-specific marker Osterix (OSX), were much higher (greater than 10 times) at basal levels in BMSCs versus ADSC and SDSC cell groups and Osteomodulin (OSM), a protein found in mature osteoblasts which links cells to the ECM, showed levels that were twice as high in BMSC cultures as well (44). Another earlier study by Jansen and colleagues found that there were large differences between the genetic profiles of ASCs derived from differing sources; human BMSCs appear to be more genetically prepared to undergo skeletal development than human ADSCs (45). With the consideration of apparent differences in gene expression in predifferentiated states of various ASCs, as well as 
unique features based solely upon harvest location and cell type, questions regarding genetic predisposal and natural capability are valid.

Although ADSCs and MDSCs can differentiate into osteoblasts in vitro, they have not been demonstrated as contributing to bone repair in vivo (46), although some controversy exists as to whether muscle may also contribute stem cells to repair. Cells derived from adipose and muscle tissues that are more accessible can potentially serve as autologous transplants. ADSCs have been expanded in vitro and tested in vivo for cartilage and bone formation (47). When transplanted in muscle, ADSCs induce ectopic bone (48). In a canine defect model, ADSCs did not have a significant effect on repair when transplanted locally even after osteogenic differentiation; however, ADSCs could augment bone regeneration after genetic modification to overexpress BMP2 (49). Shen et al. demonstrated that MDSCs expressing BMP4 could heal a critical-sized skull bone defect in immunocompetent mice; MDSCs could still be found in the repair site at 3 weeks post implantation, but were mostly gone by 4 weeks, although some of the cells appeared to differentiate into osteoblasts in the new bone (50). Thus, MDSCs and ADSCs can act mainly as carriers, producing osteogenic factors to recruit endogenous cells.

In addition to the in vitro experiments which lend support to the osteogenic success of BMSCs, in vivo studies have also proven similar conclusions. In experiments testing the ability of BMSCs to repair bone defects in the mid-diaphysis of rabbits, the BMSC treatment groups, either from an autologous or allogeneic source, were determined to be more effective in osteogenesis and bone formation in vivo (51). Sato and colleagues obtained similar results, with successful administration of BMSCs to rabbit periosteal distraction. BMSCs significantly contributed to increases in bone height, volume, mineral density, and bone mineral content (52). Success of BMSCs was not only demonstrated in animal models, but also in an earlier clinical 
study by Quarto and colleagues. They used bone marrow progenitors harvested from bone marrow and expanded the cells ex vivo to repair large bone defects in three patients. Implants were aided by macroporous hydroxyapatite scaffolds. In all patients, radiography and computed tomography confirmed successful bone-implant integration and callus formation at the repair sites (53). Just as with SDSCs and chondrogenesis, the ECM microenvironment can help dictate differentiation. A BMSC-based ECM enhanced osteogenesis of BMSCs expanded on this ECM, which seems to reflect the ASCs' capacities for differentiation toward their "intended" lineages based on their individual matrix properties as tissue-specific stem cells (54). With successful integration into the bone tissue for in vivo repair, undeniable successful differentiation, and studies suggesting their favoritism toward the osteogenic lineage in vitro, BMSCs appear to be an ideal choice for ASC osteogenesis. 


\section{1f Mechanisms of Lineage Preference}

Several studies have found proteonomic, transcriptonomic $(16,55-60)$, and epigenomic $(61,62)$ heterogeneity in stem cells from different tissues which may account for the source-dependent lineage preferences. In an effort to provide plausible mechanistic explanations for the lineage preferences of ASCs discussed above, we reviewed studies that compared the molecular properties of stem cells taken from different tissues and, when possible, discussed the significance of these differences in the context of stem cell differentiation. Given the number of recent reviews on this topic (63-65), the question has not been addressed: "Why do adult stems cells from different tissues preferentially differentiate into different lineages?", which is central to the premise of this paper. Consequently, we focused on reviewing the molecular differences between stem cells taken from different tissues, drawing parallels, where possible, to studies that have investigated the mechanistic impact of the genes, proteins, and mRNA that vary in the stem cells based on tissue of origin.

\section{Differences in Gene Expression}

The stem cells from different sources have unique genetic profiles that inherently affect their ability to differentiate along various lineages (45). Investigation of genetic differences in ASCs has revealed differences in the expression of several genes, some of which have been directly implicated in differentiation mechanisms. For instance, the expression of the osteogenic genes OSX and OPN was higher in human BMSCs than in human ADSCs, while the expression of the adipogenic genes LEPTIN and ADIPSIN was highest in ADSCs, which has led some to conclude that ASC lineage preference is affected by their tissue of origin (66). Furthermore, a comparison of human BMSCs with cord blood-derived stem cells and ADSCs demonstrated that 
expression of ALP and RUNX2 was the greatest in BMSCs at all stages of osteogenic differentiation (65). Although BMSCs expressed the highest levels of collagen I (COL1A1), osteonectin (ON), and BMP2 during osteogenic induction, it was observed that ADSCs expressed higher levels of COL1A1, ON, and BMP2 prior to differentiation, which suggests that the expression profile of "resting" stem cells is not necessarily predictive of lineage preference (67). The findings are in accord with earlier studies that, under osteogenic induction, elevated osteocalcin (OCN, an osteogenic, non-collagenous protein) levels and ALP (a ubiquitously used marker of osteogenesis) activities per DNA in rat BMSCs were observed in comparison with ADSCs; further in vivo study by subcutaneously implanting the composites of these cells and hydroxyapatite ceramics into syngeneic rats for 6 weeks demonstrated that the bone volume of BMSC composites was more than that of ADSC composites ( $\mathrm{p}<0.001$ ), quantified by microcomputed tomographic analysis (68). Moreover, Djouad et al. observed a statistically insignificant increase in the upregulation of collagen II (COL2A1) and aggrecan (ACAN) during chondrogenesis by human SDSCs relative to BMSCs, and a statistically significant increase in the upregulation of OCN and ALP during osteogenesis of human BMSCs relative to human SDSCs (16). It has also been reported that human SDSCs exhibited greater expression of platelet-derived growth factor receptor alpha (PDGFR $\alpha$ ) than human BMSCs; due to human serum containing high levels of PDGF, neutralizing PDGF decreased the proliferation of SDSCs with autologous human serum (69), while human ADSCs expressed higher levels of integral membrane protein 2A (ITM2A) than human BMSCs, and forced expression of ITM2A inhibited chondrogenesis in a murine mesenchymal stem cell line (C3H10T1/2) (70).

Noel and colleagues observed differential expression between human BMSCs and ADSCs of genes (WNT11, WNT7B, and SOX6) involved in Wnt signaling and differentiation, 
an interesting finding in light of the osteogenic function of Wnt signaling (55). Canonical Wnt signaling elevates intracellular levels of $\beta$-catenin, which transposes to the nucleus and heterodimerizes with lymphoid enhancer-binding factor/T cell factor (LEF/TCF), eventually triggering translation of genes that affect lineage choice (71), while non-canonical Wnt signaling is independent of $\beta$-catenin (65). Both, however, are widely regarded to be mostly osteogenic (72), suggesting that the differential expression of Wnt signals may help predispose BMSCs toward osteogenesis. This supposition is in line with a recent report that the signaling pathways enriched in human BMSC-TERT [transduced with human telomerase reverse transcriptase gene (hTERT)] included pathways involved in bone formation (e.g. Wnt, TGF $\beta$ ) and mitogenactivated protein kinase (MAPK) signaling while signaling pathways enriched in human ADSCs belonged to adipocyte-relevant metabolic functions (e.g. steroid hormone biosynthesis and linoleic acid metabolism) (73). This finding is in agreement with other studies of human ASCs, which have found greater expression of genes relevant to bone formation or osteoblast differentiation in BMSCs relative to ADSCs, and a higher expression of genes relevant to lipid metabolism in ADSCs relative to BMSCs $(74,75)$. The studies referenced above demonstrate that heterogeneity in gene expression exists in stem cells from different tissues, and the tissue specific profile of gene expression correlates with differentiation preference.

\section{Differences at the Epigenetic Level}

The heterogeneity of ASC epigenetics may explain the differences in gene expression among ASCs of differing origins (Figure 2). Collas and colleagues noted hypomethylation in the promoters of four adipogenic ADSC genes (76) and asked whether ASCs were pre-programmed toward a certain lineage by DNA methylation (77). At least in part, the answer to the above question appears to be in the affirmative; as Boquest et al. noted, human ADSCs are 
hypermethylated in the promoters for the myogenic differentiation gene myogenin (MYOG) and the endothelial genes CD31 and CD144 (also called vascular endothelium cadherin or CDH5), and, relative to adipogenic genes, are also hypermethylated at the promoter of the osteogenic gene osteoglycin (OGN) (76-78). Mouse BMSCs were shown to undergo demethylation and gene upregulation at the OPN promoter following mechanical stimulation (a well-recognized osteogenic stimulus) and it was hypothesized that the absence of epigenetic changes to OCN and COL1A1 promoters resulted from these regions having already been primed for osteogenesis by methylations occurring prior to mechanical stimulation (79). In human SDSCs, it was found that 10 of 11 chondrogenic genes tested were promoter hypomethylated (80), which may partially account for the preference of human SDSCs to differentiate into chondrocytes (6).

Furthermore, it was shown that promoters of osteogenic transcription factors are hypermethylated in the murine myoblast $\mathrm{C} 2 \mathrm{C} 12$ cell line relative to promoters of myogenic transcription factors, and that chemically induced demethylation enhances osteogenesis and adipogenesis of $\mathrm{C} 2 \mathrm{C} 12$ cells. Hupkes et al. postulated that DNA methylation preprogramming could underlie the default differentiation of $\mathrm{C} 2 \mathrm{C} 12$ cells toward the myogenic lineage (81). Collas described 400-700 hypermethylated genes specific to ADSCs, BMSCs, and muscle progenitor cells (MPCs) and commented that these methylation patterns might be determined by the tissue-specific stem cell niche (82).

While CpG methylation is a well-studied epigenetic modification to DNA, research indicates poor correlation between gene expression and promoter methylation, suggesting that other epigenetic mechanisms may also be important determinants for lineage preference $(77,81$, 83). Additionally, many studies have discovered general hypomethylation of lineage-specific promoter regions in mesenchymal and non-mesenchymal ASCs, regardless of origin $(61,84,85)$. 
These observations helped clarify the role of $\mathrm{CpG}$ island methylation in lineage-specific promoters; it appears that hypomethylation of these promoters is permissive, but not necessarily predictive, of lineage preference $(62,82)$.

The functional significance of methylation patterns outside promoter regions is incomplete and poorly understood. Irizarry et al. showed that most tissue-specific methylation changes do not occur in $\mathrm{CpG}$ islands, but rather in nearby "CpG island shores", and that gene expression is tightly linked with these methylation patterns (86). However, others have found that tissue-specific methylation often occurs within coding sequences or entirely downstream of known genes (87) and have postulated that such sites might contain standard methyl-sensitive repressor elements that are able to operate at a distance to silence adjacent promoters (87). Intragenic methylation may also enhance transcription of noncoding RNA (87), a theory with interesting implications in light of our growing appreciation for the roles of micro RNA (miRNA or miR) in stem cell differentiation (88-90).

Histone modifications may also play a large role in influencing the lineage preference of ASCs (91). In human BMSCs, the promoter regions of the master adipogenic transcription factor, PPARG, is histone 3 lysine 9 (H3-K9) methylated, an epigenetic modification that repressed transcription, leading Tan et al. to hypothesize, "adipogenic lineage-specific genes regulated by PPARG may be silenced by the H3-K9 hypermethylation at their promoter regions" (92). Later research indicates the promoters of 70\% of underexpressed genes in human BMSCs were indeed H3-K9 methylation enriched (93). In human ADSCs, both the permissive H3-K4M3 and repressive H3-K27M3 marks have been noted on promoters for lineage-specific genes (94), which led Collas et al. to theorize that adipogenic promoters are preprogrammed for activation upon adipogenic stimulation (83). Human BMSCs are also hypomethylated as well as H3-K4M3 
and H3-K27M3 enriched (61) and this pattern may also regulate myogenesis (95). It is believed that this "bivalent" histone modification pattern positions a cell to rapidly respond to differentiation inducing stimuli; the loss of this bivalent pattern may correspond to diminished stem cell potency and differentiation $(61,85,96,97)$.

A recent study by Ragni et al. compared the miRNA profiles of human ASCs taken from sources including bone marrow, adipose tissue, and umbilical cord blood. The authors noted that the miRNA expression patterns between ASCs from unmatched donors were mostly consistent (98). In contrast to earlier studies which found only a single miRNA, miR-424, differentially expressed between human ADSCs and BMSCs (96), Ragni and coworkers concluded that, although the miRNA expression patterns of the various ASC types are similar, there appear to be at least 20 differentially expressed miRNAs between human ADSCs and human BMSCs (66). Interestingly, they noted expression differences in several miRNAs that may be involved in lineage choice.

First, they noted that the expression of miR-135b is nearly 48 times higher in BMSCs than ADSCs (66). Studies have suggested that miR-135b was downregulated in unrestricted somatic stem cell osteogenic differentiation (99); mesenchymal stem cells from multiple myeloma patients exhibited an abnormal upregulation of miR-135b, showing meanwhile impaired osteogenic differentiation and a decrease of mothers against decapentaplegic homolog 5 (SMAD5) expression, which is the target of miR-135b involved in osteogenesis (100). As reviewed by Cook et al., SMADs 1, 5, and 8 usually transmit BMP signaling, which activates distal-less 5 (DLX5), resulting in the downstream activation of RUNX2 and OSX (63). miR-135 targeting SMAD5 could effectively inhibit osteogenesis $(88,101)$. 
Second, it was found that miR-138 was 11 times more highly expressed in BMSCs than in ADSCs (66). miR-138 has been implicated in inhibiting adipogenesis (102) as well as osteogenesis (103). Focal adhesion kinase (FAK), which regulated the osteogenesis of stem cells (104), has been identified as a target of miR-138 in human BMSCs (103). Another miRNA of interest, miR-31, was expressed at fivefold greater levels in BMSCs than in ADSCs. miR-31 downregulated the adipogenic CEBPA $(88,105)$ as well as osteogenic OSX (106). Deng and colleagues investigated the role of miR-31 in rat ADSCs and concluded that miR-31, which was suppressed by elevated Runx2 expression, inhibits osteogenesis, possibly by decreasing the translation of special AT-rich sequence-binding protein 2 (Satb2) (107), a conclusion similar to earlier work demonstrating that miR-31 was diminished in osteo-differentiated BMSCs relative to BMSCs and that transfection with antisense miR-31 increased expression of Runx 2 and BMP receptor 2 (BMPR2), promoting osteogenesis (108).

Finally, Gao et al. showed that miR-424, which was expressed 5.5 times more in ADSCs than BMSCs (66), was diminished in osteo-differentiated BMSCs, and predicted that miR-424 played a role in inhibiting osteogenesis (108). The combination of these findings suggests that, although much of the tissue-specific miRNA expression in stem cells functions to prevent the premature differentiation of these stem cells, the unique profile of different tissue-specific stem cells may also help to determine lineage preference. Ragni and colleagues concluded that differential expression of miRNA may provide a molecular explanation of stem cell niche memory (66). Although the above findings may not be sufficient to completely explain, mechanistically, the observation that stem cells from different tissues exhibit lineage preferences, it is clear that there is extensive epigenetic variability between stem cells based upon origin, and it seems likely that these differences, such as the restrictive promoter hypermethylation or the 
repression of a signaling molecule implicated in differentiation (FAK, for example), play a role in the mechanisms underlying lineage preference.

\section{Differences at the Protein Level}

There is evidence that stem cells from different tissues differ in their expression of ECM proteins and secreted factors. Researchers compared the surface proteins of stem cells from different origins and concluded that, while expression of many surface markers is similar, differences do exist. For example, CD146 was more highly expressed in human BMSCs than human ADSCs (73), and CD49d was less pronounced in adult human BMSCs than perinatal human stem cells from amniotic membrane, though this finding could be influenced by donor age (60). Further, ADSCs have been found to express CD34 after isolation, while BMSCs do not (109).

Mesenchymal stem cells' secretion and responses to soluble factors may vary depending on the tissue of origin. A comparison of human BMSCs and ADSCs revealed that, at early passages (P2-P4 or up to 14-15 in vitro population doublings), BMSCs secreted more vascular

endothelial growth factor (VEGF), stromal cell-derived factor 1 (SDF-1), monocyte chemotactic protein-1 (MCP-1), and TGF $\beta 1$ than ADSCs did (110). TGF $\beta 1$ is of particular interest given its important role in regulating stem cell differentiation. TGF $\beta 1$ signals through multiple pathways, including SMAD2/3, MAPK, and Wnt. Zhao and Hantash have provided a thorough review of TGF $\beta 1$ regulation of BMSC differentiation to which the reader is referred for a detailed discussion. In short, TGF $\beta 1$ inhibited adipogenesis in fibroblasts (and possibly BMSCs), but stimulated chondrogenesis and osteogenesis of BMSCs (111). TGF $\beta 1$ may also trigger chondrogenesis in human ADSCs (112), but the chondrogenic commitment of TGF $\beta 1$-treated 
ADSCs is delayed relative to their BMSC counterparts (113). Afizah and colleagues demonstrated that human BMSCs synthesized more GAG and collagen II following TGF 33 treatment than donor-matched ADSCs (114). It has further been shown that dexamethasone augmented the TGF $\beta 1$-induced chondrogenesis in 4-month-old bovine BMSCs, but not in 4month-old bovine SDSCs (115). Human BMSCs exhibited greater expression of HLA-DR (an MHC class II cell surface receptor encoded by the human leukocyte antigen complex on chromosome 6 region $6 \mathrm{p} 21.31$ ) than stem cells from amniotic membrane after stimulation by tumor necrosis factor alpha and interferon gamma (60). The combination of heterogeneous receptor profiles and secretomes exhibited by stem cells from different tissues may underlie the previously discussed differences in sensitivity to differentiation-inducing stimuli, while also contributing, mechanistically, to lineage preference. 


\section{1g Conclusions and Perspectives}

Stem cell therapies are undoubtedly treatment options in various areas of the biomedical field; however, each stem cell population's characteristics and source are commonly overlooked factors. Overall, we can conclude that ASCs are best suited for differentiation along their natural prospective lineages for the formation of quality bone, cartilage, adipose, and muscle tissues. The theory that various multipotent stem cell subpopulations exist within a given tissue, as well as subpopulations possessing various capacities for quality differentiation, also supports the idea that ASCs from their resident locations are the most effective contributors to a particular lineage. These populations are naturally functioning and thriving in vivo with respect to the surrounding tissues in which they exist. Although the current literature offers expansive support for this idea based on fundamental in vitro data, there is still an overall lack of in vivo studies which compare a wide variety of ASCs for multilineage differentiation capacity. In order to draw more conclusive results from in vitro experiments, it would be beneficial to utilize ASC clones; these clones can eliminate the possibility of progenitor cell heterogeneity which can skew the results of multilineage studies. Another great deficiency in current multilineage studies is the lack of in vivo and in vitro studies which investigate multilineage characteristics from the same donor. Studies of this nature could give insight into variability between organisms and, more importantly, highlight, strengthen, and uncover trends and tendencies of tissue-specific stem cells as they progress toward lineages outside their respective conventional differentiation fates.

Although studies have independently demonstrated the ability of ASCs for crossdifferentiation to other lineages, studies which compare several ASCs directly, rather than retrospectively, are generally more valuable. Direct comparison methods and using multiple cell types simultaneously can offer more direct assessment and circumvent experimental variability 
to produce more reliable conclusions regarding the differentiation potential of ASCs from separate tissues. Through our current knowledge about the specific properties of each type of ASC and future in vivo experimentation, the possibility of elucidating and revealing a cellular hierarchy for ASCs and lineage-specific differentiation is feasible.

Despite noteworthy advancement in the study of niche-specific regulation of ASC lineage preference, mechanistic research remains active. Further comparative analysis of tissue-specific miRNA expression, histone modifications to lineage-specific genes, and non-promoter methylation patterns is needed. For instance, to our knowledge, the miRNA expression profile of SDSCs has not been thoroughly compared to ADSCs or BMSCs. We believe that such studies could yield important findings, especially in light of the extensively demonstrated success in SDSC chondrogenesis studies. Additionally, it is interesting to note that tissue-specific histone modifications are far more abundant in enhancer regions than in promoter regions (116), and these modifications are made prior to cell fate commitment (117), raising the possibility that lineage preference, at least in part, is a consequence of enhancer modifications. It remains likely that tissue-specific epigenetic patterns play a role in the preference of ASCs for certain lineages (91) and such modifications may underlie the differential lineage preference of ASCs derived from various anatomical tissues. Finally, although the studies considered earlier have demonstrated that stem cells from different tissues are not identical in their responses to chemical differentiation stimuli, the molecular explanation for this observation is incomplete. It would be interesting to compare, at a molecular level, the responses of stem cells from various tissues to important differentiation factors, such as BMP, Wnt, and insulin-like growth factor (IGF), as evidence exists that stem cells from different tissues might respond in subtly different ways to the same molecular stimulus. Studies further addressing differences in signaling cascades, 
secretion of soluble factors, and matrix receptors might help elucidate the underlying molecular heterogeneity among stem cells of different tissues. The implications and impacts of such discoveries would certainly span a wide array of biomedical disciplines and help shape the future of stem cell therapy and regenerative medicine. 


\section{Acknowledgements}

The authors thank Suzanne Danley and Kayla Branyan for help in editing the manuscript. This project was partially supported by Research Grants from the AO Foundation (S-12-19P) and NIH R03 (no. 1 R03 AR062763-01A1). 


\section{References}

1. Hammerick KE, Huang Z, Sun N, Lam MT, Prinz FB, Wu JC, et al. Elastic properties of induced pluripotent stem cells. Tissue Engineering Part A. 2011;17(3-4):495-502.

2. Moroni L, Fornasari PM. Human mesenchymal stem cells: a bank perspective on the isolation, characterization and potential of alternative sources for the regeneration of musculoskeletal tissues. Journal of Cellular Physiology. 2013;228(4):680-687.

3. van der Kooy D, Weiss S. Why Stem Cells? Science. 2000;287(5457):1439-1441.

4. Kølle SF, Fischer-Nielsen A, Mathiasen AB, Elberg JJ, Oliveri RS, Glovinski PV, et al. Enrichment of autologous fat grafts with ex-vivo expanded adipose tissue-derived stem cells for graft survival: a randomised placebo-controlled trial. The Lancet. 2013;382(9898):1113-1120.

5. Yoshimura H, Muneta T, Nimura A, Yokoyama A, Koga H, Sekiya I. Comparison of rat mesenchymal stem cells derived from bone marrow, synovium, periosteum, adipose tissue, and muscle. Cell and Tissue Research. 2007;327(3):449-462.

6. Sakaguchi Y, Sekiya I, Yagishita K, Muneta T. Comparison of human stem cells derived from various mesenchymal tissues: superiority of synovium as a cell source. Arthritis \& Rheumatology. 2005;52(8):2521-2529.

7. Mochizuki T, Muneta T, Sakaguchi Y, Nimura A, Yokoyama A, Koga H, et al. Higher chondrogenic potential of fibrous synovium- and adipose synovium-derived cells compared with subcutaneous fat-derived cells: distinguishing properties of mesenchymal stem cells in humans. Arthritis \& Rheumatology. 2006;54(3):843-853.

8. Peng L, Jia Z, Yin X, Zhang X, Liu Y, Chen P, et al. Comparative analysis of mesenchymal stem cells from bone marrow, cartilage, and adipose tissue. Stem Cells and Development. 2008;17(4):761-773.

9. Xie L, Zhang N, Marsano A, Vunjak-Novakovic G, Zhang Y, Lopez MJ. In vitro mesenchymal trilineage differentiation and extracellular matrix production by adipose and bone marrow derived adult equine multipotent stromal cells on a collagen scaffold. Stem Cell Reviews. 2013;9(6):858-872. 
10. Monaco E, Bionaz M, Rodriguez-Zas S, Hurley WL, Wheeler MB. Transcriptomics comparison between porcine adipose and bone marrow mesenchymal stem cells during in vitro osteogenic and adipogenic differentiation. PloS One. 2012;7(3):3248-3258.

11. Vishnubalaji R, Al-Nbaheen M, Kadalmani B, Aldahmash A, Ramesh T. Comparative investigation of the differentiation capability of bone-marrow- and adipose-derived mesenchymal stem cells by qualitative and quantitative analysis. Cell and Tissue Research. 2012;347(2):419-427.

12. Pei M, He F, Kish VL, Vunjak-Novakovic G. Engineering of functional cartilage tissue using stem cells from synovial lining: a preliminary study. Clinical Orthopaedics Related Research. 2008;466(8):1880-1889.

13. Pei M, He F, Vunjak-Novakovic G. Synovium-derived stem cell-based chondrogenesis. Differentiation. 2008;76(10):1044-1056.

14. Pei M, He F, Boyce BM, Kish VL. Repair of full-thickness femoral condyle cartilage defects using allogeneic synovial cell-engineered tissue constructs. Osteoarthritis and Cartilage. 2009;17(6):714-722.

15. Hennig T, Lorenz H, Thiel A, Goetzke K, Dickhut A, Geiger F, et al. Reduced chondrogenic potential of adipose tissue derived stromal cells correlates with an altered TGFbeta receptor and BMP profile and is overcome by BMP-6. Journal of Cellular Physiology. 2007;211(3):682-691.

16. Djouad F, Bony C, Häupl T, Uzé G, Lahlou N, Louis-Plence P, et al. Transcriptional profiles discriminate bone marrow derived and synovium derived mesenchymal stem cells. Arthritis Research \& Therapy. 2005;7(6):1304-1315.

17. Pei M. Can synovium-derived stem cells deposit matrix with chondrogenic lineage-specific determinants? Journal of Tissue Science and Engineering. 2012;3(3):1-3.

18. Koga H, Muneta T, Nagase T, Nimura A, Ju YJ, Mochizuki T, et al. Comparison of mesenchymal tissues-derived stem cells for in vivo chondrogenesis: suitable conditions for cell therapy of cartilage defects in rabbit. Cell and Tissue Research. 2008;333(2):207-215. 
19. Lee JC, Min HJ, Park HJ, Lee S, Seong SC, Lee MC. Synovial membrane-derived mesenchymal stem cells supported by platelet-rich plasma can repair osteochondral defects in a rabbit model. Arthroscopy. 2013;29(6):1034-1046.

20. Vinardell T, Sheehy EJ, Buckley CT, Kelly DJ. A comparison of the functionality and in vivo phenotypic stability of cartilaginous tissues engineered from different stem cell sources. Tissue Engineering Part A. 2012;18(11-12):1161-1170.

21. Jones BA, Pei M. Synovium-derived stem cells: a tissue-specific stem cell for cartilage engineering and regeneration. Tissue Engineering Part B Reviews. 2012;18(4):301-311.

22. Segawa Y, Muneta T, Makino H, Nimura A, Mochizuki T, Ju YJ, et al. Mesenchymal stem cells derived from synovium, meniscus, anterior cruciate ligament, and articular chondrocytes share similar gene expression profiles. Journal of Orthopaedic Research. 2009;27(4):435-441.

23. Li J, Pei M. Cell senescence: a challenge in cartilage engineering and regeneration. Tissue Engineering Part B. 2012;18(4):270-287.

24. De Bari C, Dell'Accio F, Tylzanowski P, Luyten FP. Multipotent mesenchymal stem cells from adult human synovial membrane. Arthritis \& Rheumatology. 2001;44(8):1928-1942.

25. He F, Chen X, Pei M. Reconstruction of an in vitro tissue-specific microenvironment to rejuvenate synovium-derived stem cells for cartilage tissue engineering. Tissue Engineering Part A. 2009;15(12):3809-3821.

26. He F, Pei M. Extracellular matrix enhances differentiation of adipose stem cells from infrapatellar fat pad toward chondrogenesis. Journal of Tissue Engineering and Regenerative Medicine. 2013;7(1):73-84.

27. Rinaldi F, Perlingeiro RC. Stem cells for skeletal muscle regeneration: therapeutic potential and roadblocks. Translational Research. 2014;163(4):409-417.

28. Mitchell KJ, Pannérec A, Cadot B, Parlakian A, Besson V, Gomes ER, et al. Identification and characterization of a non-satellite cell muscle resident progenitor during postnatal development. Nature Cell Biology. 2010;12(3):257-266. 
29. Starkey JD, Yamamoto M, Yamamoto S, Goldhamer DJ. Skeletal muscle satellite cells are committed to myogenesis and do not spontaneously adopt nonmyogenic fates. Journal of Histochemistry \& Cytochemistry. 2011;59(1):33-46.

30. Seale P, Rudnicki MA. A new look at the origin, function, and "stem-cell" status of muscle satellite cells. Developmental Biology. 2000;218(2):115-124.

31. Boppart MD, De Lisio M, Zou K, Huntsman HD. Defining a role for non-satellite stem cells in the regulation of muscle repair following exercise. Frontiers in Physiology. 2013;4:310.

32. Doyle MJ, Zhou S, Tanaka KK, Pisconti A, Farina NH, Sorrentino BP, et al. Abcg2 labels multiple cell types in skeletal muscle and participates in muscle regeneration. Journal of Cell Biology. 2011;195(1):147-163.

33. Qu-Petersen Z, Deasy B, Jankowski R, Ikezawa M, Cummins J, Pruchnic R, et al. Identification of a novel population of muscle stem cells in mice: potential for muscle regeneration. Journal of Cell Biology. 2002;157(5):851-864.

34. Motohashi N, Uezumi A, Yada E, Fukada S, Fukushima K, Imaizumi K, et al. Muscle CD31(-)CD45(-) side population cells promote muscle regeneration by stimulating proliferation and migration of myoblasts. American Journal of Pathology. 2008;173(3):781-791.

35. Hawke TJ, Garry DJ. Myogenic satellite cells: physiology to molecular biology. Journal of Applied Physiology. 2001;91(2):534-551.

36. Meligy FY, Shigemura K, Behnsawy HM, Fujisawa M, Kawabata M, Shirakawa T. The efficiency of in vitro isolation and myogenic differentiation of MSCs derived from adipose connective tissue, bone marrow, and skeletal muscle tissue. In vitro Cellular \& Developmental Biology-Animal. 2012;48(4):203-215.

37. Bayati V, Hashemitabar M, Gazor R, Nejatbakhsh R, Bijannejad D. Expression of surface markers and myogenic potential of rat bone marrow- and adipose-derived stem cells: a comparative study. Anatomy \& Cell Biology. 2013;46(2):113-121.

38. Lei H, Yu B, Huang Z. Comparative analysis of mesenchymal stem cells from adult mouse adipose, muscle, and fetal muscle. Molecular Biology Reports. 2013;40(2):885-892. 
39. De Bari C, Dell'Accio F, Vandenabeele F, Vermeesch JR, Raymackers JM, Luyten FP. Skeletal muscle repair by adult human mesenchymal stem cells from synovial membrane. Journal of Cell Biology. 2003;160(6):909-918.

40. Rosset P, Deschaseaux F, Layrolle P. Cell therapy for bone repair. Orthopaedics \& Traumatology: Surgery \& Research. 2014;100(1 Suppl):S107-S112.

41. Im GI, Shin YW, Lee KB. Do adipose tissue-derived mesenchymal stem cells have the same osteogenic and chondrogenic potential as bone marrow-derived cells? Osteoarthritis Cartilage. 2005;13(10):845-853.

42. Park SH, Sim WY, Min BH, Yang SS, Khademhosseini A, Kaplan DL. Chip-based comparison of the osteogenesis of human bone marrow- and adipose tissue-derived mesenchymal stem cells under mechanical stimulation. PloS One. 2012;7(9):466-89.

43. Muraglia A, Cancedda R, Quarto R. Clonal mesenchymal progenitors from human bone marrow differentiate in vitro according to a hierarchical model. Journal of Cell Science. 2000;113(Pt 7):1161-1166.

44. Pantaleoni Andrietti A, Stewart MC. Comparative osteogenesis of equine mesenchymal stem cells isolated from bone marrow, adipose tissue and synovium. 2012.

45. Jansen BJ, Gilissen C, Roelofs H, Schaap-Oziemlak A, Veltman JA, Raymakers RA, et al. Functional differences between mesenchymal stem cell populations are reflected by their transcriptome. Stem Cells and Development. 2010;19(4):481-490.

46. Colnot C. Cell sources for bone tissue engineering: insights from basic science. Tissue Engineering Part B Reviews. 2011;17(6):449-457.

47. Levi B, Longaker MT. Concise review: adipose-derived stromal cells for skeletal regenerative medicine. Stem Cells. 2011;29(4):576-582.

48. Zheng B, Cao B, Li G, Huard J. Mouse adipose-derived stem cells undergo multilineage differentiation in vitro but primarily osteogenic and chondrogenic differentiation in vivo. Tissue Engineering. 2006;12(7):1891-1901. 
49. Li H, Dai K, Tang T, Zhang X, Yan M, Lou J. Bone regeneration by implantation of adiposederived stromal cells expressing BMP-2. Biochemical and Biophysical Research Communications. 2007;356(4):836-842.

50. Shen HC, Peng H, Usas A, Gearhart B, Cummins J, Fu FH, et al. Ex vivo gene therapyinduced endochondral bone formation: comparison of muscle-derived stem cells and different subpopulations of primary muscle-derived cells. Bone. 2004;34(6):982-992.

51. Udehiya RK, Amarpal, Aithal HP, Kinjavdekar P, Pawde AM, Singh R, et al. Comparison of autogenic and allogenic bone marrow derived mesenchymal stem cells for repair of segmental bone defects in rabbits. Research in Veterinary Science. 2013;94(3):743-752.

52. Sato K, Haruyama N, Shimizu Y, Hara J, Kawamura H. Osteogenesis by gradually expanding the interface between bone surface and periosteum enhanced by bone marrow stem cell administration in rabbits. Oral Surgery, Oral Medicine, Oral Pathology, Oral Radiology, Endodontics. 2010;110(1):32-40.

53. Quarto R, Mastrogiacomo M, Cancedda R, Kutepov SM, Mukhachev V, Lavroukov A, et al. Repair of large bone defects with the use of autologous bone marrow stromal cells. New England Journal of Medicine. 2001;344(5):385-386.

54. Pei M, He F, Kish VL. Expansion on extracellular matrix deposited by human bone marrow stromal cells facilitates stem cell proliferation and tissue-specific lineage potential. Tissue Engineering Part A. 2011;17(23-24):3067-3076.

55. Noel D, Caton D, Roche S, Bony C, Lehmann S, Casteilla L, et al. Cell specific differences between human adipose derived and mesenchymal stromal cells despite similar differentiation potentials. Experimental Cell Research. 2007;314(7):1575-1584.

56. Miranda HC, Herai RH, Thomé CH, Gomes GG, Panepucci RA, Orellana MD, et al. A quantitative proteomic and transcriptomic comparison of human mesenchymal stem cells from bone marrow and umbilical cord vein. Proteomics. 2012;12(17):2607-2617.

57. Panepucci RA, Siufi JL, Silva WA, Jr, Proto-Siquiera R, Neder L, Orellana M, et al. Comparison of Gene Expression of Umbilical Cord Vein and Bone Marrow Derived Mesenchymal Stem Cells. Stem Cells. 2004;22(7):1263-1278. 
58. Strioga M, Viswanathan S, Darinskas A, Slaby O, Michalek J. Same or not the same? Comparison of adipose tissue derived versus bone marrow derived mesenchymal stem and stromal cells. Stem Cells and Development. 2012;21(14):2724-2752.

59. Wagner W, Wein F, Seckinger A, Frankhauser M, Wirkner U, Krause U, et al. Experimental Hematology. 2005;33(11):1402-1416.

60. Wegmeyer H, Broske A, Leddin M, Kuentzer K, Nisslbeck A, Hupfeld J, et al. Mesenchymal stromal cell characteristics vary depending on their origin. Stem Cells and Development. 2013;22(19):2606-2618.

61. Sorensen A, Jacobsen B, Reiner A, Andersen IS, Collas P. Promoter DNA methylation patterns of differentiated cells are largely programmed at the progenitor stage. Molecular Biology of the Cell. 2010;21(12):2066-2077.

62. Sorensen AL, Timoskainen S, West FD, Vekterud K, Boquest AC, Ahriund-Richter L, et al. Lineage-specific promoter DNA methylation patterns segregate adult progenitor cells types. Stem Cells and Development. 2010;19(8):1257-1266.

63. Cook D, Genever P. Regulation of Mesenchymal Stem Cell Differentiation. Advances in Experimental Medicine and Biology. 2013;786:213-29.

64. Fakhry M, Hamade E, Badran B, Buchet R, Magne D. Molecular mechanisms of mesenchymal stem cell differentiation towards osteoblasts. World Journal of Stem Cells. 2013;5(4):136-148.

65. James A. Review of Signaling Pathways Governing MSC Osteogenic and Adipogenic Differentiation. Scientifica (Cairo) 2013;2013:1155-1170.

66. Ragni E, Montemurro T, Montelatici E, Lavazza C, Viganò M, Rebulla P, et al. Differential microRNA signature of human mesenchymal stem cells from different sources reveals an “environmental-niche memory" for bone marrow stem cells. Experimental Cell Research. 2013;319(10):1562-1574.

67. Shafiee A, Seyedjafari E, Soleimani M, Ahmadbeigi N, Dinarvand P, Ghaemi N. A comparison between osteogenic differentiation of human unrestricted somatic stem cells and 
mesenchymal stem cells from bone marrow and adipose tissue. Biotechnology Letters. 2011;33(6):1257-1264.

68. Hayashi O, Katsube Y, Hirose M, Ohgushi H, Ito H. Comparison of osteogenic ability of rat mesenchymal stem cells from bone marrow, periosteum, and adipose tissue. Calcified Tissue International. 2008;82(3):238-247.

69. Nimura A, Muneta T, Koga H, Mochizuki T, Suzuki K, Makino H, et al. Increased proliferation of human synovial mesenchymal stem cells with autologous human serum: comparisons with bone marrow mesenchymal stem cells and with fetal bovine serum. Arthritis \& Rheumatology. 2008;58(2):501-510.

70. Boeuf S, Börger M, Hennig T, Winter A, Kasten P, Richter W. Enhanced ITM2A expression inhibits chondrogenic differentiation of mesenchymal stem cells. Differentiation. 2009;78(23):108-115.

71. Kim W, Kim M, Jho E. Wnt/B-catenin signaling: from plasma membrane to nucleus. Biochemical Journal. 2013;450(1-2):9-21.

72. Takada I, Kouzmenko A, Kato S. Wnt and PPARg signaling in osteoblastogenesis and adipogenesis. Nature Reviews Rheumatology. 2009;5(8):442-447.

73. Al-Nbaheen M, Vishnubalaji R, Ali D, Bouslimi A, Al-Jassir F, Megges M, et al. Human stromal (mesenchymal) stem cells from bone marrow, adipose tissue and skin exhibit differences in molecular phenotype and differentiation potential. Stem Cell Review. 2013;9(1):32-43.

74. Boeuf S, Richter W. Chondrogenesis of mesenchymal stem cells: role of tissue source and inducing factors. Stem Cell Research \& Therapy. 2010;1(4):31.

75. Liu TM, Martina M, Hutmacher DW, Hui JH, Lee EH, Lim B. Identification of common pathways mediating differentiation of bone marrow and adipose tissue-derived human mesenchymal stem cells into three mesenchymal lineages. Stem Cells. 2007;25(3):750-760.

76. Noer A, Sorensen AL, Boquest AC, Collas P. Stable CpG hypomethylation of adipogenic promoters in freshly isolated cultured and differentiated mesenchymal stem cells from adipose tissue. Molecular Biology of the Cell. 2006;17(8):3543-3556. 
77. Boquest A, Noer A, Collas P. Epigenetic programming of mesenchymal stem cells from human adipose tissue. Stem Cell Review. 2006;2(4):319-329.

78. Boquest AC, Noer A, Sorensen AL, Vekterud K, Collas P. CpG methylation profiles of endothelial cell specific gene promoter regions in adipose tissue stem cells suggest limited differentiation protential towards the endothelial cell lineage. Stem Cells. 2007;25(4):852-861.

79. Arnsdorf EJ, Tummala P, Castillo AB, Zhang F, Jacobs CR. The epigenetic mechanism of mechanically induced osteogenic differentiation. Journal of Biomechanics. 2010;43(15):28812886.

80. Ezura Y, Sekiya I, Koga H, Muneta T, Noda M. Methylation status of CpG islands in the promoter regions of signature genes during chondrogenesis of human synovium derived mesenchymal stem cells. Arthritis \& Rheumatology. 2009;60(5):1416-1426.

81. Hupkes M, Someren EP, Middelkamp SH, Piek E, van Zoelen EJ, Dechering KJ. DNA methylation restricts spontaneous multi-lineage differentiation of mesenchymal progenitor cells, but is stable during growth factor-induced terminal differentiation. Biochimica Biophysica Acta. 2011;1813(5):839-849.

82. Collas P. Programming differentiation potential in mesenchymal stem cell. Epigenetics. 2010;5(6):476-482.

83. Collas P, Noer A, Sorensen A. Epigenetic basis for the differentiation potential of mesenchymal and embryonic stem cells. Transfusion Medicine and Hemotherapy. 2008;35(3):205-215.

84. Mohn F, Schubeler D. Genetics and epigenetics: stability and plasticity during cellular differentiation. Trends in Genetics. 2009;25(3):129-136.

85. Reik W. Stability and flexibility of epigenetic gene regulation in mammalian development. Nature. 2007;447(7143):425-432.

86. Irizarry R, Ladd-Acosta C, Wen B, Wu Z, Montano C, Onyango P, et al. The human colon cancer methylome shows similar hypo- and hypermethylation at conserved tissue-specific $\mathrm{CpG}$ island shores. Nature Genetics. 2009;41(2):178-186. 
87. Straussman R, Nejman D, Roberts D, Steinfeld I, Blum B, Benvenisty N, et al.

Developmental programming of $\mathrm{CpG}$ island methylation profiles in the human genome. Nature Structural \& Molecular Biology. 2009;16(5):564-571.

88. Guo L, Zhao R, Wu Y. The role of microRNAs in self renewal and differentiation of mesenchymal stem cells. Experimental Hematology. 2011;39(6):608-616.

89. Perdiguero E, Sousa-Victor P, Ballestar E, Muñoz-Cánoves P. Epigenetic regulation of myogenesis. Epigenetics. 2009;4(8):541-550.

90. Romao J, Jin W, Dodson M, Hausman GJ, Moore SS, Guan LL. MicroRNA regulation in mammalian adipogenesis. Experimental Biology and Medicine. 2011;236(9):997-1004.

91. Teven C, Liu X, Hu N, Tang N, Kim SH, Huang E, et al. Epigenetic regulation of mesenchymal stem cells: a focus on osteogenic and adipogenic differentiation. Stem Cells International. 2011;2011:201371.

92. Tan J, Huang H, Huang W, Li L, Guo J, Huang B, et al. The genomic landscapes of histone H3-Lys9 modifications of gene promoter regions and expression profiles in human bone marrow mesenchymal stem cells. Journal of Genetics \& Genomics. 2008;35(10):585-593.

93. Tan J, Lu J, Huang W, Dong Z, Kong C, Li L, et al. Genome-wide analysis of histone H3 lysine9 modifications in human mesenchymal stem cell osteogenic differentiation. PLoS One. 2009;4:e6792.

94. Noer A, Linderman L, Collas P. Histone H3 modifications associated with differentiation and long term culture of mesenchymal adipose stem cells. Stem Cells and Development. 2009;18(5):725-736.

95. Dilworth FJ, Blais A. Epigenetic regulation of satellite cell activation during muscle regeneration. Stem Cell Research Therapy. 2011;2(2):18.

96. Aranda P, Agirre X, Ballestar E, Andreu EJ, Román-Gómez J, Martin-Subero JI, et al. Epigenetic signatures associated with different levels of differentiation potential in human stem cells. PLoS One. 2009;4(11):7809. 
97. Voigt P, Tee W, Reinberg D. A double take on bivalent promoters. Genes \& Development. 2013;27(12):1318-1338.

98. Pandey AC, Semon JA, Kaushal D, O’Sullivan RP, Glowacki J, Gimble JM, et al. MicroRNA profiling reveals age dependent differential expression of nuclear factor $\mathrm{kB}$ and mitogen activated protein kinase in adipose and bone marrow-derived human mesenchymal stem cells. Stem Cell Research Therapy. 2011;2(6):49.

99. Schaap-Oziemlak AM, Raymakers RA, Bergevoet SM, Glissen C, Jansen BJ, Adema GJ, et al. MicroRNA has-miR-135b regulates mineralization in osteogenic differentiation of human unrestricted somatic stem cells. Stem Cells and Development. 2010;19(6):877-885.

100. Xu S, Santini GC, Veirman KD, Broek IV, Leleu X, Becker AD, et al. Upregulation of miR-135b is involved in the impaired osteogenenic differentiation of mesenchymal stem cells derived from multiple myeloma patients. PLoS One. 2013;8(11):e79752.

101. Li Z, Hassan MQ, Volinia S, van Wijnen AJ, Stein JL, Croce CM, et al. A microRNA signature for a BMP2 induced osteoblast lineage commitment program. Proceedings of the National Academy of Sciences USA. 2008;105(37):13906-13911.

102. Yang Z, Bian C, Zhou H, Huang S, Wang S, Liao L, et al. MicroRNA has-miR-138 inhibits adipogenic differentiation of human adipose tissue derived mesenchymal stem cells through adenovirus EID-1. Stem Cells and Development. 2011;20(2):259-267.

103. Eskildsen T, Taipaleenmaki H, Stenvang J, Abdallah BM, Ditzel N, Nossent AY, et al. MicroRNA-138 regulates osteogenic differentiation of human stromal (mesenchymal) stem cells in vivo. Proceedings of the National Academy of Sciences USA. 2011;108(15):6139-6144.

104. Mathieu P, Loboa E. Cytoskeletal and focal adhesion influences on mesenchymal stem cell shape, mechanical properties, and differentiation down osteogenic, adipogenic, and chondrogenic pathways. Tissue Engineering Part B. 2012;18(6):436-444.

105. Sun F, Wang J, Pan Q, Yu Y, Zhang Y, Wan Y, et al. Characterization of function and regulation of miR-24-1 and miR-31. Biochemical and Biophysical Research Communications. 2009;380(3):660-665. 
106. Baglìo SR, Devescovi V, Granchi D, Baldin N. MicroRNA expression profiling of human bone marrow mesenchymal stem cells during osteogenic differentiation reveals Osterix regulation by miR-31. Gene. 2013;527(1):321-331.

107. Deng Y, Zhou H, Zou D, Xie Q, Bi X, Gu P, et al. The role of miR-31-modified adipose tissue derived stem cells in repairing rat critical sized calvarial defects. Biomaterials. 2013;34(28):6717-6728.

108. Gao J, Yang T, Han J, Yan K, Qui X, Zhou Y, et al. MicroRNA expression during osteogenic differentiation of human multipotent mesenchymal stromal cells from bone marrow. Journal of Cellular Biochemistry. 2011;112(7):1844-1856.

109. Bourin P, Bunnell BA, Casteilla L, Dominici M, Katz AJ, March KL, et al. Stromal cells from the adipose tissue-derived stromal vascular fraction and culture expanded adipose tissuederived stromal/stem cells: a joint statement of the International Federation for Adipose Therapeutics and Science (IFATS) and the International Society for Cellular Therapy (ISCT) Cytotherapy. 2013;15(6):641-648.

110. Dmitrieva RI, Minullina IR, Bilibina AA, Tarasova OV, Anisimov SV, Zaritskey AY. Bone marrow and subcutaneous adipose tissue derived mesenchymal stem cells: Differences and similarities. Cell Cycle. 2012;11(2):377-383.

111. Zhao L, Hantash B. TGF-B1 regulates differentiation of bone marrow mesenchymal stem cells. Vitamins \& Hormones. 2011;87:127-141.

112. Erickson GR, Gimble JM, Franklin DM, Rice HE, Awad H, Guilak F. Chondrogenic potential of adipose tissue derived stromal cells in vitro and in vivo. Biochemical and Biophysical Research Communications. 2002;290(2):763-769.

113. Mehlhorn AT, Niemeyer P, Kaiser S, Finkenzeller G, Stark GB, Sudkamp NP, et al. Differential expression pattern of extracellular matrix molecules during chondrogenesis of mesenchymal stem cells from bone marrow and adipose tissue. Tissue Engineering. 2006;12(10):2853-2862. 
114. Afizah H, Yang Z, Hui JH, Ouyang HW, Lee EH. A comparison between the chondrogenic potential of human bone marrow stem cells (BMSCs) and adipose-derived stem cells (ADSCs) taken from the same donors. Tissue Engineering. 2007;13(4):659-666.

115. Shintani N, Hunziker EB. Differential effects of dexamethasone on the chondrogenesis of mesenchymal stromal cells: influence of microenvironment, tissue origin and growth factor. European Cells and Materials. 2011;22:302-319.

116. Heintzman N, Hon G, Hawkins R, Kheradpour P, Stark A, Harp LF, et al. Histone modifications at human enhancers reflect global cell type specific gene expression. Nature. 2009;459(7243):108-112.

117. Ong CT, Corces VG. Enhancers: emerging roles in cell fate specification. EMBO Reports. 2012;13(5):423-430. 
Figure 1.1.1:

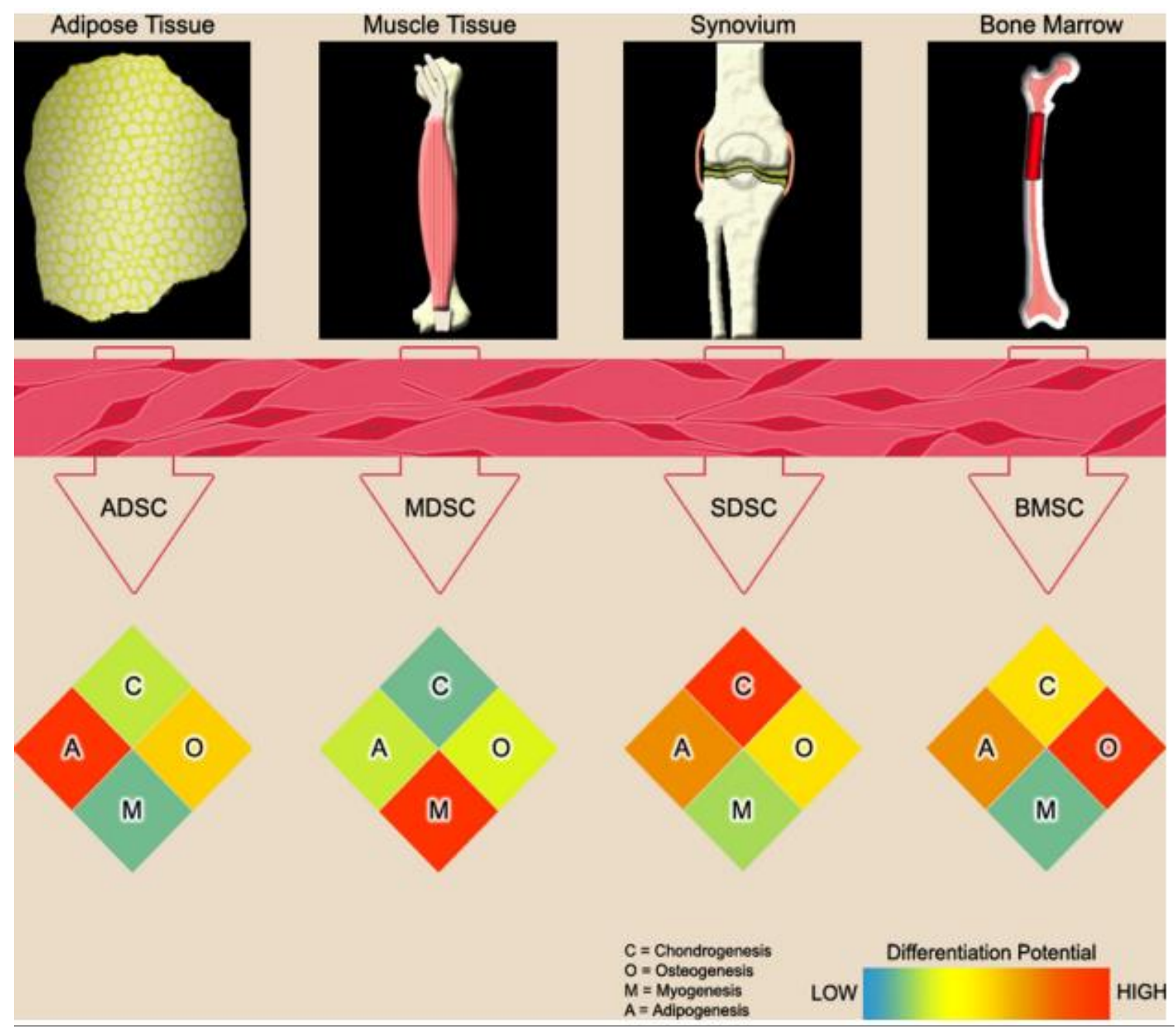


Fig. 1.1.1 Adult stem cells can be derived from various tissues in the body. These viable and undifferentiated stem cell populations can be expanded in vitro and induced to undergo lineagespecific differentiation for chondrogenesis $(\mathrm{C})$, osteogenesis $(\mathrm{O})$, myogenesis $(\mathrm{M})$, or adipogenesis (A). Although the cells may appear similar in morphology upon harvest, they are anything but identical. From the data presented in the section "Tissue Specific Stem Cells Benefiting LineageSpecific Differentiation", the efficacy of ASCs in lineage-specific differentiation is greatly affected by the type of resident tissue from which they are harvested. In the heatmap, the differentiation capacity is visualized by color ranging from low differentiation (blue) to high differentiation (red). 
Figure 1.1.2:

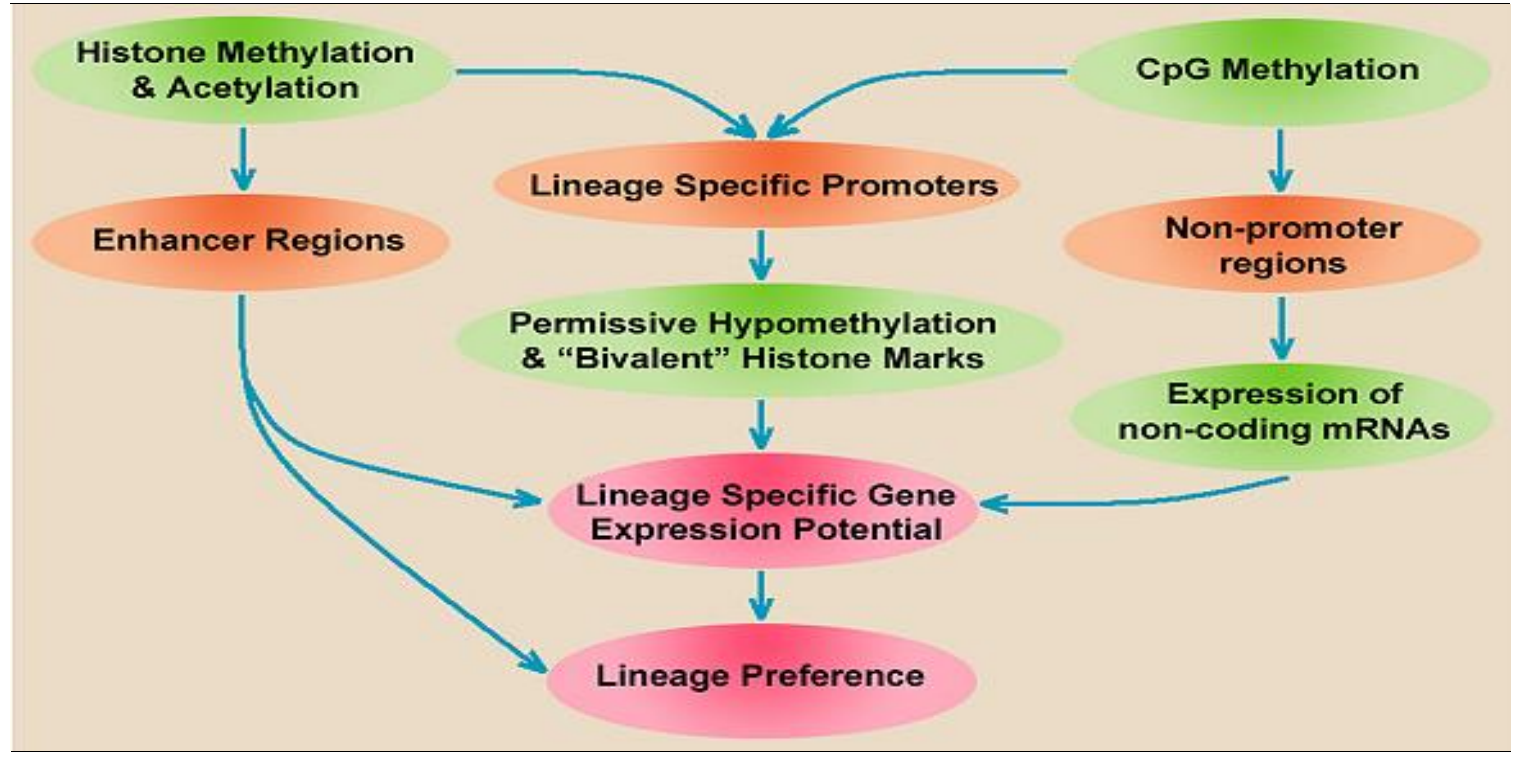


Fig. 1.1.2 Epigenetic determination of niche-specific lineage preference. $\mathrm{CpG}$ methylation of promoters creates a permissive, but non-predictive state, while non-promoter $\mathrm{CpG}$ methylation, histone modifications, and differentially expressed miRNAs may combine to determine lineage preference. 


\section{Chapter 1.2}

\section{The Intervertebral Disc \& Nucleus Pulposus}




\subsection{The intervertebral disc}

\section{Tissue Morphology}

The nucleus pulposus (NP) tissue is located at the innermost spaces of each intervertebral disc (IVD) of the spinal column. The mature NP tissue and NP cells (NPCs), which compose the tissue, are similar to chondrocytes, and have been previous described as "chondrocyte-like cells" [1] [2]. The IVDs themselves lie between each vertebrae, and should be viewed as dynamic, heterogeneous structures that are designed to respond to dynamic mechanical loading cycles integral to disc function [3], ultimately allowing for the complex movements of humans such as twisting and bending [4] [5]. The outer structure of the IVD that encompasses the NP tissue is called the annulus fibrosis (AF), which is by definition a ring of fibrocartilage [5]. This helps to add structural support to the disc and contain the inner NP tissue. Together, the NP and AF, as well as their anatomical relationship with the adjacent cartilage end plate (CEP) and bone end plate (BEP) form the basic anatomical structure known as the intervertebral disc (IVD) (Fig. 1) [4].

The development of the mature NP tissues and parallels between NP and other chondrocytic tissues have been extensively reviewed previously [6] [7]. Briefly, mature NP cells arise from cells of the developing notochord. These notochordal cells ultimately allow for proliferation of chondrocytelike cells and the stimulation of proteoglycan expression [7] [8]. Brachyury (T), transforming growth factor-beta TGF $\beta$, Noggin (Nog), and several factors others contribute to the development of the mature NP phenotype as well [9]. Early IVD degeneration may be partially initiated by the loss of these regulatory notochordal cells and the associated paracrine factors seem to have roles in the disc, proteoglycan synthesis, and overall disc integrity [10] [11]. 
Figure 1.2.1:

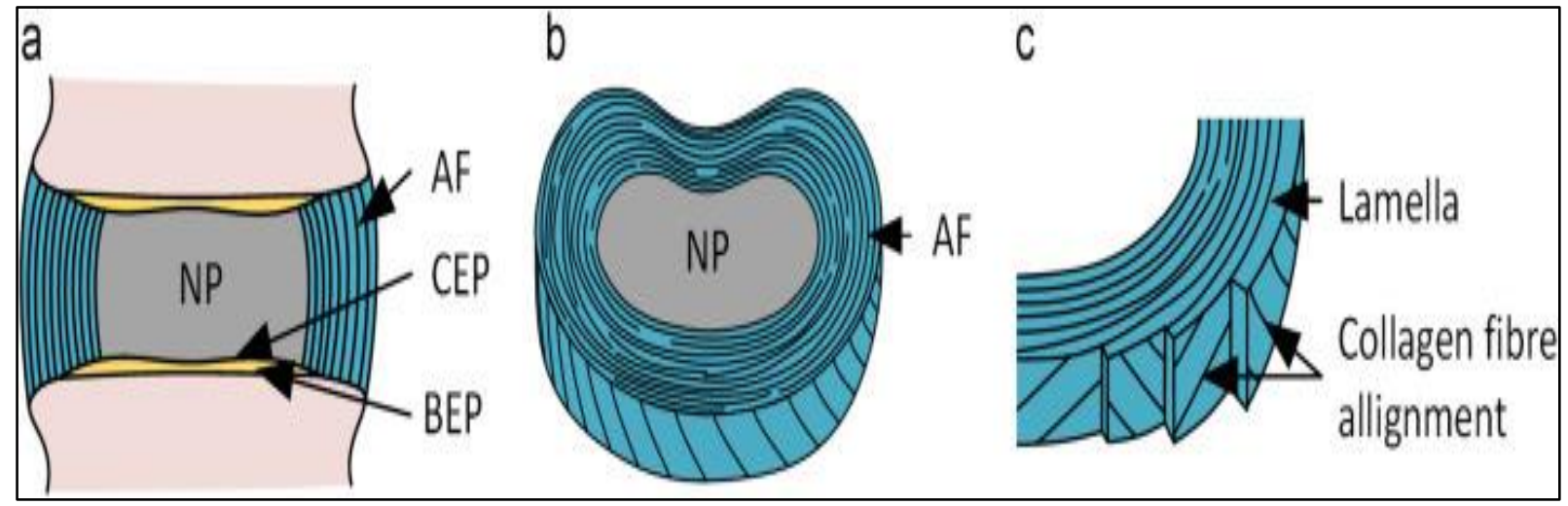

Figure 1.2.1 Gross anatomy of a disc. (A) Cross section of a disc in the coronal plane, (B) diagram of a transversely sliced IVD and (C) diagram showing the alternating fibre alignment in successive lamellae. AF: anulus fibrosus; CEP: cartilaginous endplate; BEP: bony endplate; NP: nucleus pulposus.

[4] N. Newell, JP Little, A. Christou, MA Adams, CJ Adam, SD Masouros.

Biomechanics of the human intervertebral disc: A review of testing techniques and results. Journal of the Mechanical Behavior of Biomedical Materials, Volume 69, 2017, 420-434.

Intervertebral Disc Degeneration (IVDD)

Nearly three decades ago, Humzah and Soames described the IVD as a tissue capable of selfmaintenance and "considerable regenerative properties", and that IVDs could be dramatically affected by changes to the disc structure, resulting in downstream effects on vertebral column kinematics [5]. This IVD degeneration has been previously linked to low back pain [1] [6] and sciatica [11] [12], representing a significant socioeconomic burden [13]. Furthermore, early literature by Urban and Roberts noted that IVDs are quite susceptible to degeneration at an accelerated pace compared to other cartilaginous tissues, leading to complete loss of function in 
the most severe cases [2]. Haefeli and colleagues have reported that degeneration can occur very early life, at the second decade or even before [14] and unlike any other musculoskeletal tissue, suffers from extensive age-related degeneration [15]. Unfortunately, once degeneration occurs, the regeneration capacity and ability to regenerate NP tissue without external intervention is a significant challenge [16]. Part of the IVD aging process and IVDD- associated degenerative progression occurs as a function of disc tissue degradation due to the inflammatory microenvironment [17], decreased protein solubility and insoluble type I collagen accumulation [11] and upregulation of catabolic matrix metalloproteases [17] [18] and multitude of factors destabilizing disc and matrix homeostasis. This process is typically associated with IVDD, but degeneration can also occur following acute injury such as an intervertebral disc herniation [19] [20] [21] [22], often informally referred to as a "slipped disc." 
Figure 1.2.2:

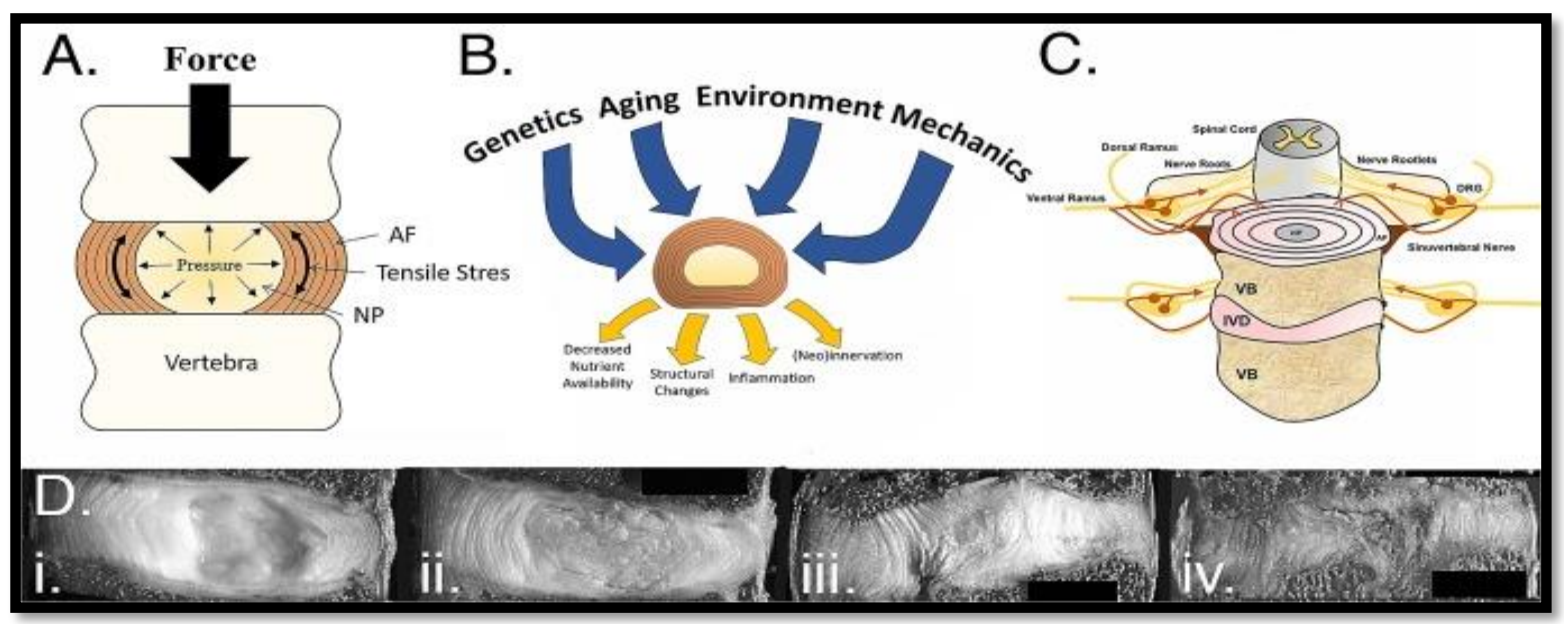

Figure 1.2.2 Schematic of the intervertebral disc and anatomic sub-structures. Abbreviations:

AF, anulus fibrosus; IVD, intervertebral disc; NP, nucleus pulposus. (A) Forces of load bearing and motion contribute to axial loading of the IVD that is balanced by a hydrostatic pressure generated in the gelatinous NP. Pressure in the NP gives rises to a tensile stress in the surrounding AF or a "hoop stress" as depicted. (B) Multiple factors contribute to the onset of degeneration and matrix changes in the IVD, including aging, wear and tear of daily loading, inheritance patterns, and environmental factors such as $\mathrm{pH}$, oxygen, and nutrient supply. (C) Anatomic changes in the substructures of the IVD can give rise to pain or disability due to proximity of the IVD with major sensory and motor nerves. Age- or pathology-related changes can contribute to an increased innervation of the IVD by sensory neurons branching from the dorsal root ganglion that are a purported cause of discogenic pain. (D) Disc degeneration is classified based on anatomic changes that may be distinguished by imaging appearance or gross morphology, as shown here. (i) Healthy

[22] Robert D. Bowles, Lori A. Setton. Biomaterials for intervertebral disc regeneration and repair. Biomaterials, Volume 129, 2017, 54-67. 
young adult disc with a defined NP. (ii) Middle age adult disc that is slightly aged but not yet degenerated. (iii) Moderately degenerated young adult disc. (iv) Severely degenerated young adult disc.

[22] Robert D. Bowles, Lori A. Setton. Biomaterials for intervertebral disc regeneration and

repair. Biomaterials, Volume 129, 2017, 54-67.

\section{IVD and cell-based therapies}

Because IVDD can lead to disc herniation and tears in the AF and the generation of herniated NP fragments, protrusions, and prolapses then can lead to nerve root pressure and pain [23] [24], making treatment is often a necessary step for patients. There is a wide-range of strategies for treatment of disc herniation and IVDD, ranging from conservative and non-surgical methods such as spinal manipulation [25] [26] [27] and minimally-invasive surgical microdiscectomy [28] [29] to more invasive surgeries such as spinal fusion [30] [31].

While many of these treatment options address structural concerns of the IVD, it is also increasingly important to consider the regenerative potential and compromised tissue environment of the disc. Cell-based therapies aim to address these concerns by replacing the lost cellularity of the NP space [32] [33]. Although repopulating the tissue with cells is an excellent strategy, it can be an initial challenge to harvest the necessary cell numbers due to limitations in replicative lifespan and cells with a healthy phenotype and regenerative potential to contribute to tissue regeneration for use in therapeutic interventions [34] [35] [36]. Preconditioning strategies, including hypoxia, extracellular matrix expansion, and growth factor supplementation aim to address these concerns to increase cell numbers and redifferentiation potential [37] [38] [39]. With 
these factors in mind, and as a part of this dissertation, we aimed to compare emerging methods of cellular preconditioning with potential implications in future therapies seeking to improve NP regeneration and IVD repair. 


\section{References}

[1] Risbud MV, Schoepflin ZR, Mwale F, Kandel RA, Grad S, Iatridis JC, Sakai D, Hoyland JA. Defining the phenotype of young healthy nucleus pulposus cells: recommendations of the Spine Research Interest Group at the 2014 annual ORS meeting. J Orthop Res. 2015 Mar;33(3):283-93.

[2] Urban JP, Roberts S. Development and degeneration of the intervertebral discs. Mol Med Today. 1995 Oct;1(7):329-35.

[3] Neidlinger-Wilke C, Galbusera F, Pratsinis H, Mavrogonatou E, Mietsch A, Kletsas D, Wilke HJ. Mechanical loading of the intervertebral disc: from the macroscopic to the cellular level. Eur Spine J. 2014 Jun;23 Suppl 3:S333-43.

[4] Newell N, Little JP, Christou A, Adams MA, Adam CJ, Masouros SD. Biomechanics of the human intervertebral disc: A review of testing techniques and results. J Mech Behav Biomed Mater. 2017 May;69:420-434.

[5] Humzah MD, Soames RW. Human intervertebral disc: structure and function. Anat Rec. 1988 Apr;220(4):337-56.

[6] Smith LJ, Nerurkar NL, Choi KS, Harfe BD, Elliott DM. Degeneration and regeneration of the intervertebral disc: lessons from development. Dis Model Mech. 2011 Jan;4(1):31-41.

[7] Chen S, Fu P, Wu H, Pei M. Meniscus, articular cartilage and nucleus pulposus: a comparative review of cartilage-like tissues in anatomy, development and function. Cell Tissue Res. 2017 Apr 17.

[8] Erwin WM, Ashman K, O'Donnel P, Inman RD. Nucleus pulposus notochord cells secrete connective tissue growth factor and up-regulate proteoglycan expression by intervertebral disc chondrocytes. Arthritis Rheum. 2006 Dec;54(12):3859-67.

[9] Chan WC, Au TY, Tam V, Cheah KS, Chan D. Coming together is a beginning: the making of an intervertebral disc. Birth Defects Res C Embryo Today. 2014 Mar;102(1):83-100.

[10] Hunter CJ, Matyas JR, Duncan NA. The functional significance of cell clusters in the notochordal nucleus pulposus: survival and signaling in the canine intervertebral disc. Spine (Phila Pa 1976). 2004 May 15;29(10):1099-104.

[11] Yee A, Lam MP, Tam V, Chan WC, Chu IK, Cheah KS, Cheung KM, Chan D. Fibrotic-like changes in degenerate human intervertebral discs revealed by quantitative proteomic analysis. Osteoarthritis Cartilage. 2016 Mar;24(3):503-13. 
[12] Harris RI, Macnab I. Structural changes in the lumbar intervertebral discs; their relationship to low back pain and sciatica. J Bone Joint Surg Br. 1954 May;36-B(2):30422.

[13] Katz JN. Lumbar disc disorders and low-back pain: socioeconomic factors and consequences. J Bone Joint Surg Am. 2006 Apr;88 Suppl 2:21-4.

[14] Haefeli M, Kalberer F, Saegesser D, Nerlich AG, Boos N, Paesold G. The course of macroscopic degeneration in the human lumbar intervertebral disc. Spine (Phila Pa 1976). 2006 Jun 15;31(14):1522-31.

[15] Buckwalter JA. Aging and degeneration of the human intervertebral disc. Spine (Phila Pa 1976). 1995 Jun 1;20(11):1307-14.

[16] Huang YC, Leung VY, Lu WW, Luk KD. The effects of microenvironment in mesenchymal stem cell-based regeneration of intervertebral disc. Spine J. 2013 Mar;13(3):352-62.

[17] Ouyang A, Cerchiari AE, Tang X, Liebenberg E, Alliston T, Gartner ZJ, Lotz JC. Effects of cell type and configuration on anabolic and catabolic activity in 3D co-culture of mesenchymal stem cells and nucleus pulposus cells. J Orthop Res. 2017 Jan;35(1):6173. doi: 10.1002/jor.23452.

[18] Millward-Sadler SJ, Costello PW, Freemont AJ, Hoyland JA. Regulation of catabolic gene expression in normal and degenerate human intervertebral disc cells: implications for the pathogenesis of intervertebral disc degeneration. Arthritis Res Ther. 2009;11(3):R65.

[19] Xu H, Mei Q, Xu B, Liu G, Zhao J. Expression of matrix metalloproteinases is positively related to the severity of disc degeneration and growing age in the East Asian lumbar disc herniation patients. Cell Biochem Biophys. 2014 Nov;70(2):1219-25.

[20] Schroeder JE, Dettori JR, Brodt ED, Kaplan L. Disc degeneration after disc herniation: are we accelerating the process? Evid Based Spine Care J. 2012 Nov;3(4):3340.

[21] Sun D, Liu P, Cheng J, Ma Z, Liu J, Qin T. Correlation between intervertebral disc degeneration, paraspinal muscle atrophy, and lumbar facet joints degeneration in patients with lumbar disc herniation. BMC Musculoskelet Disord. 2017 Apr20;18(1):167.

[22] Bowles RD, Setton LA. Biomaterials for intervertebral disc regeneration and repair. Biomaterials. 2017 Jun;129:54-67.

[23] Yasuma T, Koh S, Okamura T, Yamauchi Y. Histological changes in aging lumbar intervertebral discs. Their role in protrusions and prolapses. J Bone Joint Surg Am. 1990 Feb;72(2):220-9.

[24] Hurri H, Karppinen J. Discogenic pain. Pain. 2004 Dec;112(3):225-8. 
[25] Ehrler M, Peterson C, Leemann S, Schmid C, Anklin B, Humphreys BK. Symptomatic, MRI Confirmed, Lumbar Disc Herniations: A Comparison of Outcomes Depending on the Type and Anatomical Axial Location of the Hernia in Patients Treated With High-Velocity, Low-Amplitude Spinal Manipulation. J Manipulative Physiol Ther. 2016 Mar-Apr;39(3):192-9.

[26] Kuo PP, Loh ZC. Treatment of lumbar intervertebral disc protrusions by manipulation. Clin Orthop Relat Res. 1987 Feb;(215):47-55.

[27] Zhao P, Tian Q. [The history and principle of spinal manipulation in the treatment of lumbar intervertebral disc herniation]. Zhongguo Gu Shang. 2009 Apr;22(4):276-8.

[28] Phan K, Dunn AE, Rao PJ, Mobbs RJ. Far lateral microdiscectomy: a minimallyinvasive surgical technique for the treatment of far lateral lumbar disc herniation. J Spine Surg. 2016 Mar;2(1):59-63.

[29] Koban O, Öğrenci A, Yaman O, Dalbayrak S. Phantom Radicular Pain Treated with Lumbar Microdiscectomy: A Case Report. Turk Neurosurg. 2017 Feb 15.

[30] Willems P. Decision making in surgical treatment of chronic low back pain: the performance of prognostic tests to select patients for lumbar spinal fusion. Acta Orthop Suppl. 2013 Feb;84(349):1-35.

[31] Dower A, Chatterji R, Swart A, Winder MJ. Surgical management of recurrent lumbar disc herniation and the role of fusion. J Clin Neurosci. 2016 Jan;23:44-50.

[32] Leckie SK, Sowa GA, Bechara BP, Hartman RA, Coelho JP, Witt WT, Dong QD, Bowman BW, Bell KM, Vo NV, Kramer BC, Kang JD. Injection of human umbilical tissue-derived cells into the nucleus pulposus alters the course of intervertebral disc degeneration in vivo. Spine J. 2013 Mar;13(3):263-72.

[33] Kovac D, Rotim K, Kovac V, Perović D, Kvesić D, Cerina V, Bozić B, Ivanec Z, Radić I, Cimić M, Lakicevic G, Borić M, Petrović T. [New techniques and prospects in invasive treatment in vertebrology]. Lijec Vjesn. 2011 Mar-Apr;133(3-4):125-32.

[34] Pei M, Shoukry M, Li J, Daffner SD, France JC, Emery SE. Modulation of in vitro microenvironment facilitates synovium-derived stem cell-based nucleus pulposus tissue regeneration. Spine (Phila Pa 1976). 2012 Aug 15;37(18):1538-47.

[35] Wang J, Tao Y, Zhou X, Li H, Liang C, Li F, Chen QX. The potential of chondrogenic pre-differentiation of adipose-derived mesenchymal stem cells for regeneration in harsh nucleus pulposus microenvironment. Exp Biol Med (Maywood). 2016 Dec;241(18):2104-2111.

[36] Lee JS, Lee SM, Jeong SW, Sung YG, Lee JH, Kim KW. Effects of age, replicative lifespan and growth rate of human nucleus pulposus cells on selecting age 
range for cell-based biological therapies for degenerative disc diseases. Biotech Histochem. 2016 Jul;91(5):377-85.

[37] Pei M. Environmental preconditioning rejuvenates adult stem cells' proliferation and chondrogenic potential. Biomaterials. 2017 Feb;117:10-23.

[38] Pizzute T, Li J, Zhang Y, Davis ME, Pei M. Fibroblast Growth Factor Ligand Dependent Proliferation and Chondrogenic Differentiation of Synovium-Derived Stem Cells and Concomitant Adaptation of Wnt/Mitogen-Activated Protein Kinase Signals. Tissue Eng Part A. 2016 Aug;22(15-16):1036-46.

[39] Zhang Y, Pizzute T, Li J, He F, Pei M. sb203580 preconditioning recharges matrix-expanded human adult stem cells for chondrogenesis in an inflammatory environment - A feasible approach for autologous stem cell based osteoarthritic cartilage repair. Biomaterials. 2015 Sep;64:88-97. 


\section{Chapter 1.3}

\section{Wnt signaling pathways}




\section{Wnt Signaling Pathways}

\section{Canonical wht signaling}

Canonical signaling relies on the molecule $\beta$-catenin for downstream signaling and is a wellconserved cellular pathway in humans and other animals [1]. Several other key molecules, such as axin, APC, CKI $\alpha$, glycogen synthase kinase $3 \beta$ are also involved in canonical signaling, and are responsible for combining with one another to form a "destruction complex," which allows for the phosphorylation of $\beta$-catenin to occur in the absence of a canonical Wnt ligand, ultimately leading to its protease-dependent degradation in the cell cytoplasm [1] [2] [3]. When a stimulating Wnt ligand is present, it is bound to a transmembrane receptor called Frizzled (FZD) and its associated low density receptor protein, LRP5/6 to transmit the signal, recruiting intracellular disheveled (Dvl) [4]. This leads to the cytoplasmic sequestration of both Axin and glycogen synthase kinase, preventing the formation of the "destruction complex," allowing the persistence and accumulation of $\beta$-catenin in the cell nucleus to modulate gene expression [2] [4]. (Fig 1.3.1)

\section{Nonanonical wnt signaling}

In noncanonical signaling, signaling occurs through different groups or individual Wnt signals than canonical signals, acting in mechanisms independent of $\beta$-catenin [5] Another major difference in the two branches of Wnt signaling is that the noncanonical signaling requires FZD, but does not rely on LRP, or $\beta$-catenin [5]. One noncanonical pathway mechanism is known as the Planar Cell Polarity (PCP) pathway, controlling tissue polarity [6], and is regulated through small GTPases to promote actin cytoskeleton reorganization [7]. The other noncanonical mechanism is the $\mathrm{Wnt} / \mathrm{Ca}^{2+}$ pathway, in which calcium release activates protein kinase $\mathrm{C}$ (PKC) 
and calmodulin-dependent kinase II [8] , inhibiting canonical signaling. Because of the several mechanisms in place allowing for Wnt signal transduction to both occur and carry out its own regulation, it may be most accurately viewed as a set of non-distinct pathways, with consideration for the simultaneous and heavily integrated inputs at the receptor and intracellular levels of activation [8] (Fig 1.3.1)

\section{Roles for wnt signaling}

Interestingly, recent literature has suggested that crosstalk between canonical and noncanonical Wnt pathways can occur, where noncanonical signals can serve as "fine-tuning" signals to regulate canonical $\beta$-catenin/TCF activity [9]. Also, crosstalk between Wnt pathways, mitogen activated protein kinase (MAPK) signaling, and in processes regulating cell proliferation, homeostasis, and tissue development have been reviewed in cartilage regeneration and chondrogenesis [10]. In addition to the regulatory roles and crosstalk with other established pathways, Wnt signaling pathways have been implicated in the self-renewal of stem cell populations [11] [12] [13] as well as regulation of cancer, including the progression, dormancy, immunity, and maintenance of cancer stem cells [14]. With the multifaceted roles of canonical and noncanonical signals across many tissue types, and the crosstalk with other pathways, it seems that Wnt signaling plays a central and vital role in cell metabolism.

Although many biochemical signaling pathways are responsible for the regulation of cell metabolism and stem cell differentiation, both canonical and non-canonical Wnt/ $\beta$-catenin pathways are potential regulators of NP redifferentiation. These are well-conserved pathways that have been extensively studied and implicated in tissue homeostasis [15], NPC apoptosis [16] as well as cell migration and differentiation [17], with particularly notable roles in 
chondrogenesis [18] [19] [20]. Wnt/ $\beta$-catenin pathway signals have been previously implicated in IVDD of canine discs [21] as well as disc aging, tissue degeneration, and proper development of murine IVD tissues [22] [23] [24]. More recently, Wnt (Wingless protein family) signaling abnormalities have also been associated with intervertebral disc ossification and subsequent disc inflammation [25]. Winkler and colleagues found that canonical Wnt and Shh signaling were decreased in the aging adult human IVD, and suggested that the Wnt pathway could be a potential therapeutic target for disc degeneration therapy [26]. Although several studies have suggested that Wnt signals play key roles in human disc, there are much fewer studies using human-derived IVDs or NPCs to fully support such hypotheses, especially studies that directly compare multiple Wnt signals at once during NPC redifferentiation. Furthermore, recently published studies have suggested that the progression of degenerative disc disease may be related to the communication and crosstalk between Wnt and TGF-Beta signaling pathways [27] [28], a well-known differentiation stimulating signal, occurring naturally in cartilaginous tissues, and commonly used to perpetuate the chondrogenic and redifferentiated NP phenotypes in vitro [29] [30]. Additionally, Wnt3A, Wnt5A, and Wnt11 have each been shown to be involved in mesenchymal stem cell (MSC) differentiation. Wnt3A has been implicated in MSC proliferation, increasing differentiation potential, and suppression of differentiation [31] [32] [33] [34], while Wnt5A and Wnt11 have been more extensively implicated in differentiation and cartilage development [20] [35] [36] [37] [38] [39].

With well-identified roles of Wnt signaling in cartilage and stem cell differentiation [10] [20] [35] as well as the inherent similarities and shared properties between the chondrogenic differentiation of chondrocytic tissues [40] the relatively ubiquitous roles in stem cell differentiation, we hypothesized that both canonical and noncanonical signals could serve as 
potential regulators for nucleus pulposus cell redifferentiation in humans. With a greater understanding for the roles of both canonical WNT3A and noncanonical WNT5A and WNT11 ligands in NP tissue, it may be possible to modulate these signals in the future to increase redifferentiation capacity of harvested cells for regenerative therapies. 


\section{References}

[1] Koury J, Zhong L, Hao J. Targeting Signaling Pathways in Cancer Stem Cells for Cancer Treatment. Stem Cells Int. 2017;2017:2925869.

[2] Stamos JL, Weis WI. The $\beta$-catenin destruction complex. Cold Spring Harb Perspect Biol. 2013 Jan 1;5(1):a007898.

[3] Clevers H. Wnt/beta-catenin signaling in development and disease. Cell. 2006 Nov 3;127(3):469-80.

[4] MacDonald BT, Tamai K, He X. Wnt/beta-catenin signaling: components, mechanisms, and diseases. Dev Cell. 2009 Jul;17(1):9-26.

[5] Tamura M, Nemoto E. Role of the Wnt signaling molecules in the tooth. Jpn Dent Sci Rev. 2016 Nov;52(4):75-83.

[6] Gray RS, Roszko I, Solnica-Krezel L. Planar cell polarity: coordinating morphogenetic cell behaviors with embryonic polarity. Dev Cell. 2011 Jul 19;21(1):12033.

[7] Schlessinger K, Hall A, Tolwinski N. Wnt signaling pathways meet Rho GTPases. Genes Dev. 2009 Feb 1;23(3):265-77.

[8] van Amerongen R, Nusse R. Towards an integrated view of Wnt signaling in development. Development. 2009 Oct;136(19):3205-14.

[9] Fan J, Wei Q, Liao J, Zou Y, Song D, Xiong D, Ma C, Hu X, Qu X, Chen L, Li L, Yu Y, Yu X, Zhang Z, Zhao C, Zeng Z, Zhang R, Yan S, Wu T, Wu X, Shu Y, Lei J, Li Y, Zhang W, Haydon RC, Luu HH, Huang A, He TC, Tang H. Noncanonical Wnt signaling plays an important role in modulating canonical Wnt-regulated stemness, proliferation and terminal differentiation of hepatic progenitors. Oncotarget. 2017 April 18;8(16):27105-27119.

[10] Zhang Y, Pizzute T, Pei M. A review of crosstalk between MAPK and Wnt signals and its impact on cartilage regeneration. Cell Tissue Res. 2014 Dec;358(3):63349.

[11] Yan KS, Janda CY, Chang J, Zheng GXY, Larkin KA, Luca VC, Chia LA, Mah AT, Han A, Terry JM, Ootani A, Roelf K, Lee M, Yuan J, Li X, Bolen CR, Wilhelmy J, Davies PS, Ueno H, von Furstenberg RJ, Belgrader P, Ziraldo SB, Ordonez H, Henning SJ, Wong MH, Snyder MP, Weissman IL, Hsueh AJ, Mikkelsen TS, Garcia KC, Kuo CJ. 
Non-equivalence of Wnt and R-spondin ligands during Lgr5(+) intestinal stem-cell selfrenewal. Nature. 2017 May 3.

[12] Bao X, Lian X, Hacker TA, Schmuck EG, Qian T, Bhute VJ, Han T, Shi M, Drowley L, Plowright A, Wang QD, Goumans MJ, Palecek SP. Long-term self-renewing human epicardial cells generated from pluripotent stem cells under defined xeno-free conditions. Nat Biomed Eng. 2016;1.

[13] Shen J, Chen X, Jia H, Meyers C, Shrestha S, Asatrian G, Ding C, Tsuei R, Zhang X, Péault B, Ting K, Soo C, James AW. Effects of WNT3A and WNT16 on the osteogenic and adipogenic differentiation of perivascular stem cells. Tissue Eng Part A. 2017 May 2.

[14] Shang S, Hua F, Hu ZW. The regulation of $\beta$-catenin activity and function in cancer: therapeutic opportunities. Oncotarget. 2017 Feb 25.

[15] Arai F, Hiyama A, Sakai D, Yokoyama K, Mochida J. The expression and role of non-canonical (PKC) signaling in nucleus pulposus cell metabolism. J Orthop Res. 2012 Sep;30(9):1478-85.

[16] Wang J, Chen H, Cao P, Wu X, Zang F, Shi L, Liang L, Yuan W. Inflammatory cytokines induce caveolin- $1 / \beta$-catenin signalling in rat nucleus pulposus cell apoptosis through the p38 MAPK pathway. Cell Prolif. 2016 Jun;49(3):362-72.

[17] Garcin CL, Habib SJ. A Comparative Perspective on Wnt/ $\beta$-Catenin Signalling in Cell Fate Determination. Results Probl Cell Differ. 2017;61:323-350.

[18] Green JD, Tollemar V, Dougherty M, Yan Z, Yin L, Ye J, Collier Z, Mohammed MK, Haydon RC, Luu HH, Kang R, Lee MJ, Ho SH, He TC, Shi LL, Athiviraham A. Multifaceted signaling regulators of chondrogenesis: Implications in cartilage regeneration and tissue engineering. Genes Dis. 2015 Dec;2(4):307-327.

[19] Zhong L, Huang X, Karperien M, Post JN. The Regulatory Role of Signaling Crosstalk in Hypertrophy of MSCs and Human Articular Chondrocytes. Int J Mol Sci. 2015 Aug 14;16(8):19225-47.

[20] Pizzute T, Li JT, Zhang Y, Pei M. FGF ligand dependent proliferation and multidifferentiation of synovium-derived stem cells and concomitant adaptation of Wnt/MAPK signals during chondrogenesis. Tissue Eng Part A 2016;22(15-16):1036-46.

[21] Smolders LA, Meij BP, Onis D, Riemers FM, Bergknut N, Wubbolts R, Grinwis GC, Houweling M, Groot Koerkamp MJ, van Leenen D, Holstege FC, Hazewinkel HA, Creemers LB, Penning LC, Tryfonidou MA. Gene expression profiling of early 
intervertebral disc degeneration reveals a down-regulation of canonical Wnt signaling and caveolin-1 expression: implications for development of regenerative strategies. Arthritis Res Ther. 2013 Jan 29;15(1):R23.

[22] Dahia CL, Mahoney EJ, Durrani AA, Wylie C. Intercellular signaling pathways active during intervertebral disc growth, differentiation, and aging. Spine (Phila Pa 1976). 2009 Mar 1;34(5):456-62.

[23] Kondo N, Yuasa T, Shimono K, Tung W, Okabe T, Yasuhara R, Pacifici M, Zhang Y, Iwamoto M, Enomoto-Iwamoto M. Intervertebral disc development is regulated by Wnt/ $\beta$-catenin signaling. Spine (Phila Pa 1976). 2011 Apr 15;36(8):E513-8.

[24] Jia H, Ma J, Lv J, Ma X, Xu W, Yang Y, Tian A, Wang Y, Sun L, Xu L, Fu L, Zhao J. Oestrogen and parathyroid hormone alleviate lumbar intervertebral disc degeneration in ovariectomized rats and enhance Wnt/ $\beta$-catenin pathway activity. Sci Rep. 2016 Jun 9;6:27521.

[25] Shao J, Yu M, Jiang L, Wu F, Liu X. Sequencing and bioinformatics analysis of the differentially expressed genes in herniated discs with or without calcification. Int $\mathbf{J}$ Mol Med. 2017 Jan;39(1):81-90.

[26] Winkler T, Mahoney EJ, Sinner D, Wylie CC, Dahia CL. Wnt signaling activates Shh signaling in early postnatal intervertebral discs, and re-activates Shh signaling in old discs in the mouse. PLoS One. 2014;9(6):e98444.

[27] Cleary MA, van Osch GJ, Brama PA, Hellingman CA, Narcisi R. FGF, TGF $\beta$ and Wnt crosstalk: embryonic to in vitro cartilage development from mesenchymal stem cells. J Tissue Eng Regen Med. 2015 Apr;9(4):332-42.

[28] Matta A, Karim MZ, Isenman DE, Erwin WM. Molecular Therapy for Degenerative Disc Disease: Clues from Secretome Analysis of the Notochordal Cell-Rich Nucleus Pulposus. Sci Rep. 2017 Mar 30;7:45623.

[29] Chen WH, Lo WC, Lee JJ, Su CH, Lin CT, Liu HY, Lin TW, Lin WC, Huang TY, Deng WP. Tissue-engineered intervertebral disc and chondrogenesis using human nucleus pulposus regulated through TGF-beta1 in platelet-rich plasma. J Cell Physiol. 2006 Dec;209(3):744-54.

[30] Cai F, Zhu L, Wang F, Shi R, Xie XH, Hong X, Wang XH, Wu XT. The Paracrine Effect of Degenerated Disc Cells on Healthy Human Nucleus Pulposus Cells Is Mediated by MAPK and NF- $\kappa$ B Pathways and Can Be Reduced by TGF- $\beta 1$. DNA Cell Biol. 2017 Feb;36(2):143-158.

[31] Lee HC, Lim S, Han JY. Wnt/ $\beta$-catenin signaling pathway activation is required for proliferation of chicken primordial germ cells in vitro. Sci Rep. 2016 Sep 30;6:34510. 
[32] Narcisi R, Arikan OH, Lehmann J, Ten Berge D, van Osch GJ. Differential Effects of Small Molecule WNT Agonists on the Multilineage Differentiation Capacity of Human Mesenchymal Stem Cells. Tissue Eng Part A. 2016 Nov;22(21-22):1264-1273.

[33] Narcisi R, Cleary MA, Brama PA, Hoogduijn MJ, Tüysüz N, ten Berge D, van Osch GJ. Long-term expansion, enhanced chondrogenic potential, and suppression of endochondral ossification of adult human MSCs via WNT signaling modulation. Stem Cell Reports. 2015 Mar 10;4(3):459-72.

[34] Buchtova M, Oralova V, Aklian A, Masek J, Vesela I, Ouyang Z, Obadalova T, Konecna Z, Spoustova T, Pospisilova T, Matula P, Varecha M, Balek L, Gudernova I, Jelinkova I, Duran I, Cervenkova I, Murakami S, Kozubik A, Dvorak P, Bryja V, Krejci P. Fibroblast growth factor and canonical WNT/ $\beta$-catenin signaling cooperate in suppression of chondrocyte differentiation in experimental models of FGFR signaling in cartilage. Biochim Biophys Acta. 2015 May;1852(5):839-50.

[35] Hosseini-Farahabadi S, Geetha-Loganathan P, Fu K, Nimmagadda S, Yang HJ, Richman JM. Dual functions for WNT5A during cartilage development and in disease. Matrix Biol. 2013 Jun 24;32(5):252-64.

[36] Zhu X, Zhu H, Zhang L, Huang S, Cao J, Ma G, Feng G, He L, Yang Y, Guo X. Wls-mediated Wnts differentially regulate distal limb patterning and tissue morphogenesis. Dev Biol. 2012 May 15;365(2):328-38.

[37] Thorfve A, Dehne T, Lindahl A, Brittberg M, Pruss A, Ringe J, Sittinger M, Karlsson C. Characteristic Markers of the WNT Signaling Pathways Are Differentially Expressed in Osteoarthritic Cartilage. Cartilage. 2012 Jan;3(1):43-57.

[38] Liu S, Zhang E, Yang M, Lu L. Overexpression of Wnt11 promotes chondrogenic differentiation of bone marrow-derived mesenchymal stem cells in synergism with TGFß. Mol Cell Biochem. 2014 May;390(1-2):123-31.

[39] Usami Y, Gunawardena AT, Iwamoto M, Enomoto-Iwamoto M. Wnt signaling in cartilage development and diseases: lessons from animal studies. Lab Invest. 2016 Feb;96(2):186-96.

[40] Shoukry M, Li J, Pei M. Reconstruction of an in vitro niche for the transition from intervertebral disc development to nucleus pulposus regeneration. Stem Cells Dev. 2013 Apr 15;22(8):1162-76. 
Figure 1.3.1:

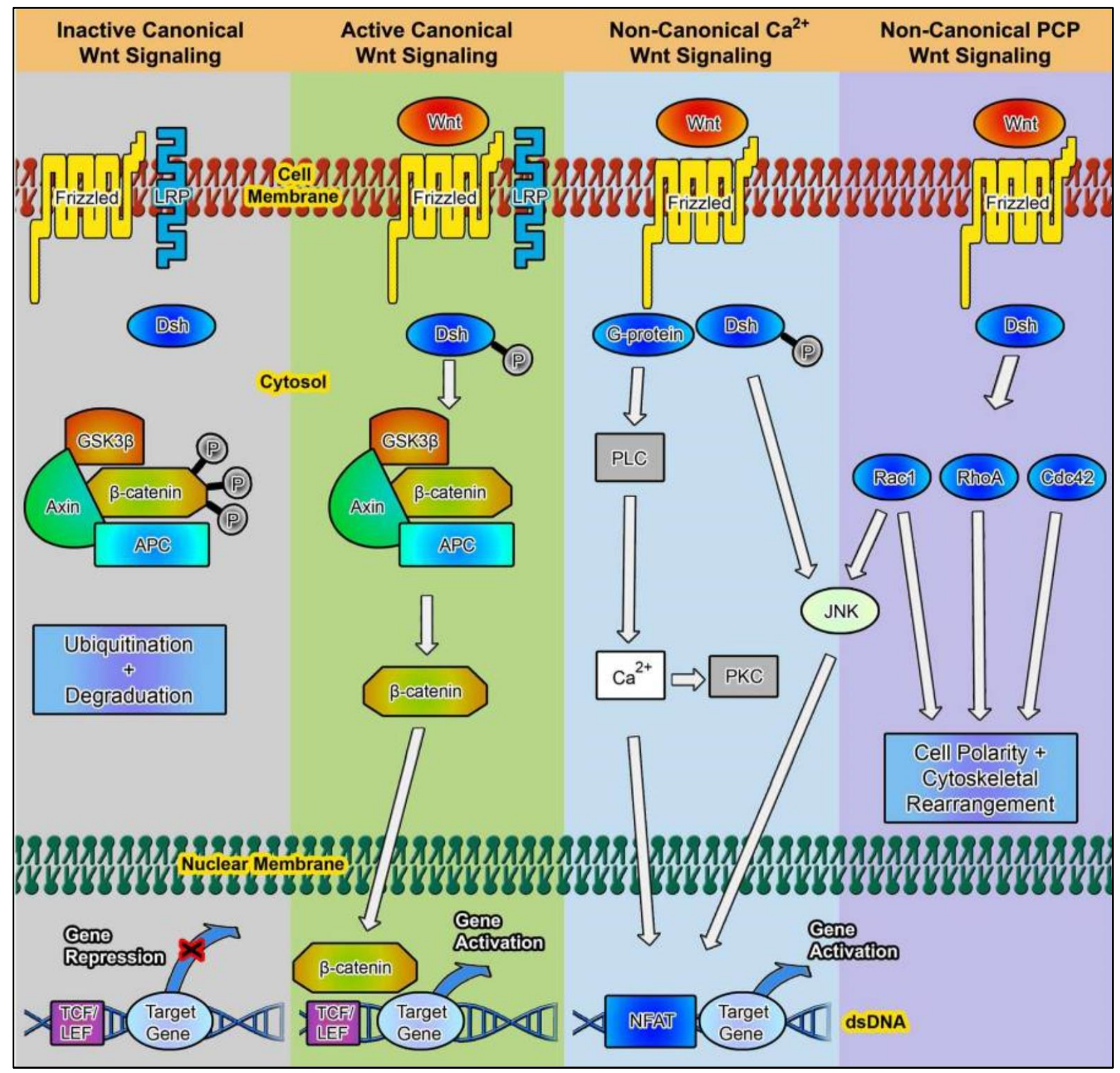

Fig 1.3.1 Three Wnt-dependent pathways have been categorized: canonical Wnt/ $\beta$-catenin and non-canonical Wnt/PCP as well as $\mathrm{Wnt} / \mathrm{Ca} 2+$ pathways. Canonical Wnt/ $\beta$-catenin pathway: In cells, with an inactive state of canonical Wnt signaling, cytosolic $\beta$-catenin is targeted to Zhang Y, Pizzute T, Pei M. A review of crosstalk between MAPK and Wnt signals and its impact on cartilage regeneration. Cell Tissue Res. 2014 Dec;358(3):633-49. 
proteolytic degradation through phosphorylation by the APC-Axin-GSK3 $\beta$ complex and further ubiquitination through action of $\beta$ TrCP-dependent E3 ubiquitin ligase complex. On stimulation by Wnt ligands through binding to Fzd receptors and its co-receptor Lrp, Fzd recruits and phosphorates Dsh, and inhibits APC-Axin-GSK3 $\beta$ complex formation by the recruitment and inhibition of GSK3 $\beta$. Consequently, $\beta$-catenin can accumulate in the cytoplasm and enter the nucleus, activating transcription of target genes through association with the Lef1/Tcf transcription factor family. Non-canonical Wnt/Ca2+ pathway: Interaction of Wnt ligands with Fzd receptors can lead to an increase in intracellular calcium level, through possibly the activation of phospholipase C (PLC). Intracellular calcium will subsequently activate $\mathrm{Ca} 2+/$ calmodulin-dependent protein kinase II (CAMKII) and protein kinase $\mathrm{C}(\mathrm{PKC})$ in cells, as well as the transcription factor NFAT. This pathway is particularly important for convergentextension movements during gastrulation. Additionally, Fzd receptors can also activate JNK, promoting expression of specific genes through activation of AP-1. Non-canonical Wnt/PCP pathway: This pathway is characterized by an asymmetric distribution of Fzd and related receptors, resulting in the polarization of the cell. Also, Wnt-signaling activates Cdc42, RhoA, and Rac1 leading to cytoskeleton rearrangement. Rac1 can also activate JNK, activating specific gene transcription through modulation of the AP-1 protein complex.

Zhang Y, Pizzute T, Pei M. A review of crosstalk between MAPK and Wnt signals and its impact on cartilage regeneration. Cell Tissue Res. 2014 Dec;358(3):633-49. 


\section{Chapter 1.4}

\section{Preconditioning strategies for nucleus pulposus rejuvenation and impacts on cell immunity}

In preparation for submission; TBD 2017

Tyler Pizzute ${ }^{1}$, Zachary Werner ${ }^{2}$, Ming Pei ${ }^{1}$

${ }^{1}$ Stem Cell and Tissue Engineering Laboratory, Department of Orthopaedics, West Virginia University, One Medical Center Drive, PO Box 9196, Morgantown, WV 26506-9196, USA.

${ }^{2}$ West Virginia University School of Medicine, One Medical Center Drive, PO Box 9196, Morgantown, WV 26506-9196, USA.

Running Title: Nucleus Pulposus Preconditioning and Immunity

Corresponding author: Ming Pei, MD, PhD

Stem Cell and Tissue Engineering Laboratory, Department of Orthopaedics, West Virginia University, PO Box 9196, One Medical Center Drive, Morgantown, WV 26506-9196, USA, Telephone: 304-293-1072; Fax: 304-293-7070; Email: mpei@ @sc.wvu.edu 


\begin{abstract}
Intervertebral discs are susceptible to acute injuries and degenerative diseases that frequently lead to persistent back pain and significant morbidity. At the cellular level, the nucleus pulposus (NP) cells located at the inner disc space undergo changes that ultimately result in deleterious consequences for self-repair of the affected NP tissue, particularly evident following injury or severe disruption of the disc microenvironment. Recently, cell preconditioning strategies have been shown to rejuvenate NP cells for successful redifferentiation; however, the impact of such strategies on immunity has not been extensively emphasized. This review highlights the emerging strategies that utilize fibroblast growth factor 2 (FGF-2) media supplementation and threedimensional (3D) growth substrates and presents potential immunological effects resulting from preconditioning applications. Ultimately, it is imperative that preconditioning methods aimed at increasing redifferentiation and regenerative capacity of cells need to preserve immune tolerance. Prospective areas of interest for the preliminary detection of immune issues in preconditioned cells are also briefly discussed.
\end{abstract}




\section{Introduction}

Disease of the intervertebral disc and acute injuries such as disc herniation lead to significant patient morbidity including disc degeneration and back pain, causing significant socioeconomic burden (Ito \& Creemers, 2013). One of the most common procedures to repair discs following herniation is surgical discectomy of the herniated nucleus pulposus (NP) tissue; however, a wide range of complications accompanied by generally unfavorable or dissatisfying outcomes are frequent occurrences with the procedure (Belykh et al., 2016). Additionally, another major concern surrounding disc disease and herniation is the loss of disc height (Gunzburg et al. 1992; Rohlmann et al., 2006; Sakai \& Grad, 2015), accompanied by matrix decomposition, decrease in cellularity, and disc dehydration (Sakai \& Grad, 2015). Unfortunately, degenerative discs become largely unbalanced in their anabolic and catabolic signals for matrix regulation, further perpetuated by the release of inflammatory factors in the disc (Wuertz et al., 2012). These conditions can make it challenging for NP tissue in the degenerative disc to heal or to rejuvenate the existing cell population, which typically degenerates further without proper intervention.

Promising repair strategies that utilize the patients' own cells (autologous cells) to stabilize the disc microenvironment and repopulate it with new NP cells is an attractive solution; however, it requires the patients' cells to be rejuvenated to an improved condition to beneficially contribute to the repair process. Rejuvenation can potentially be achieved through in vitro preconditioning of autologous NP cells to increase their proliferative and redifferentiation capacities for future therapeutic applications. "Preconditioning" is an encompassing term for the various growth strategies utilized to increase overall cell health, morphology, proliferation rates, and, for NP cells, their redifferentiation potential. 
Although a number of cell preconditioning studies focused on NP repair have been performed, the majority do not address potential immune concerns of preconditioning strategies. Interestingly, despite the intervertebral disc's status as an immune privileged site (Takada et al., 2002; Ma et al., 2015), compromised and degenerated discs have exhibited noticeable changes in the immunophenotypes of cells residing in the disc tissues; these include increases in Fas (APO1/CD95) and decreases in CD178 (Fas ligand) in degenerated or herniated discs (Ma et al., 2015). Furthermore, preconditioning strategies used to increase redifferentiation and proliferative potential could pose unidentified immune concerns similar to those observed in the degenerative disc.

Despite the multitude of potential options for preconditioning strategies to increase cell potency and overall rejuvenation (Pei 2017), this review focuses primarily on the emerging and more popular preconditioning strategies for NP regeneration and other chondrocytic tissues, including media supplementation with fibroblast growth factor 2 (FGF-2) and the use of threedimensional (3D) growth substrates and potential immune concerns arising from preconditioning methods. 


\section{FGF-2 Preconditioning}

Because expansion of NP and chondrocytic cells in monolayer culture alone can cause dedifferentiation, such as decreasing proliferation rate and loss of the NP phenotype with serial passaging (Martin et al., 1999), supplementation with cell culture media serves as one potential preconditioning approach to improve the quality of harvested cells. Fibroblast growth factor 2 (FGF-2), also known as basic fibroblast growth factor, has been shown to increase cell proliferation in many cell types (Ellman et al., 2008); for example, recent studies have demonstrated its potential use as a preconditioning supplement for NP cell expansion. Tsai and colleagues tested serially passaged porcine NP cells in the presence of FGF-2, reporting increased expression of sulfated proteoglycans, an important matrix component in NP tissue, as well as slowed turnover for aggrecan compared to untreated controls. Interestingly, FGF-2 treatment resulted in a dose-dependent reduction of actin stress fiber formation (Tsai et al., 2007) which, when increased, is typically associated with a decreased ability of chondrocytic cells to reexpress their differentiated phenotype (Martin et al., 1999; Tsai et al., 2007). Another study comparing various preconditioning strategies in NP cells harvested from degenerated human discs revealed that FGF-2 preconditioning increased cell proliferation in NP cells from moderately degenerated discs. After preconditioning, cells were redifferentiated in pellet culture and exposed to TGF-beta3 differentiated media. FGF-2 preconditioned NP cells from moderately degenerated discs exhibited increases in ACAN (aggrecan) expression but decreased COL1A1 (type I collagen) expression after 21 days of redifferentiation (Pizzute et al., 2017).

Although these studies support plausible applications for FGF-2 as a preconditioning stimulus, fewer studies have investigated the potential roles of FGF-2 to modulate immune responses (Liu et al., 2015) or alter immune responses to cells preconditioned with FGF-2. A recent 
study investigating the association of cytosolic FGF-2 with RIG-I, a protein associated with detection and signaling responses to viral infections and inflammatory cytokine production, reported that cytosolic FGF-2 mediated the reduction of type 1 interferon production and had an unexpected role in innate immunity (Liu et al., 2015). HLA-DR (major histocompatibility complex II) expression in preconditioned cells is another area of interest given its well-established role in T-cell mediated immune responses and antigen presentation. A study by Dighe and colleagues found that passaging of human bone marrow mesenchymal stem cell cultures in the presence of FGF-2 did not significantly correlate to HLA-DR expression; rather, duration of culture maintenance was an influential factor in HLA-DR expression (Dighe et al., 2013). A similar result was reported by Pizzute and colleagues in a human NP cell preconditioning study following application of $10 \mathrm{ng} / \mathrm{mL}$ of FGF-2, with no significant increases in HLA-DR expression versus controls (Pizzute et al., 2017).

Another noteworthy surface marker involved in cell-mediated apoptosis is the Fas (APO1/CD95) receptor (Westendorp et al., 1995), which is thought to contribute to immune privilege in the intervertebral disc (Sun et al., 2013; Wang et al., 2007) and in caspase-mediated apoptosis (Fu et al., 2016; Imai et al., 1999). This pathway could pose a serious threat to preconditioned cells and their utilization in regenerative applications in the disc since CD95 expression could make them susceptible targets of Fas-mediated apoptosis by infiltrating cytotoxic T-cells (Stich et al., 2015). In a recent preconditioning study, it was reported that human NP cells supplemented with $10 \mathrm{ng} / \mathrm{mL}$ of FGF-2 were able to significantly decrease CD95 surface expression versus untreated controls (Pizzute et al., 2017). To date, few studies have examined the effects of preconditioning on CD95 expression; however, it could serve as a potential predictor of NP survival and tolerance in the disc. 
An alternative to the overlying goals of many cancer cell studies, where success is typically derived from the death of targeted cancer cells, regenerative therapies benefit from the survival of administered cells for repair of damaged or depleted tissues. With this notion at hand, it may be reasonable to suggest that parallels can be drawn between the two areas of study, where a cell survival mechanism represents a hurdle for one, but can be beneficially exploited for the other. In a study investigating FGF-2 treated mouse fibroblasts and tumor immunotherapy, Li and colleagues reported that cells treated with $100 \mathrm{ng} / \mathrm{mL}$ of FGF-2 in immunocompetent mice exhibited immunoprotective effects, but not with untreated fibroblasts. Results included delayed onset of tumor growth, which largely contributed to an antibody-mediated autoimmune response to both FGF-2-activiated fibroblasts and tumor cells ( $\mathrm{Li}$ et al., 2014). Interestingly, another cancer study also implicated an FGF-2-dependent survival pathway, the FGFR1/PKC $\delta / E R K$ effector pathway, and API5 gene expression as a means for tumoral immunity. Ultimately, this pathway results in the downstream degradation of the proapoptotic molecule, BIM, which was also reported to be dependent upon FGF-2 secretion (Noh et al., 2014). This same pathway and receptor were reported in bovine NP cells, with FGF-2 acting through the receptor FGFR1, ultimately implicating FGF-2 in matrix homeostasis and intervertebral disc degeneration (Li et al., 2008). Other studies have reported similar results, with FGF-2 naturally released during injury of cartilage and in chondrocytic tissues (Vincent et al., 2002; Li et al., 2008). Since recent literature suggests that FGF-2 pathways play a role in tumoral immunity, similar biochemical mechanisms may be involved in FGF-2 preconditioning; however, more studies using NP and chondrocytic cells are necessary to validate this hypothesis.

Although few studies directly implicate FGF-2 as a major immune regulator, it seems reasonable to suggest that FGF-2 plays a background role in immune tolerance and apoptosis, and 
could even potentially provide protective effects for preconditioned cells. Future studies more completely elucidating the role of FGF-2 preconditioning on immune tolerance in cultured cells are necessary and could provide new clinically relevant data to complement the already welldemonstrated applications of FGF-2 supplementation as an expansion strategy for cell-based regenerative therapies. 


\section{Three-dimensional substrate preconditioning}

Culturing chondrocytic cells in an environment that can prevent cell flattening and help express differentiation genes is important to maintain the cells' phenotype (Martin et al., 1999) and also serves as an alternative approach to monolayer culture. Although many 3D culture procedures can provide a preconditioning environment to increase the differentiation and proliferative capacities of seeded cells (Pei 2017), the concern that culturing cells in a 3D substrate, especially those derived from biological origins, may alter immunogenic properties of expanded cells, which has recently been hypothesized (Stich et al., 2015; Zhang et al., 2015). A notable study by Stich and colleagues compared human mild and severely degenerated cervical NP, evaluated their regenerative properties, and assayed the resulting immunogenic responses following 3D-culture (Stich et al., 2015). Their results indicated that 3D fibrin/poly (lactic-coglycolic) acid culture resulted in increased proliferation of immune cells following $3 \mathrm{D}$ expansion, as indicated by the co-culture and analysis of labeled peripheral blood mononuclear cells (PBMCs) in a mixed lymphocyte reaction (MLR). Furthermore, the authors noted an increase in this trend with severely degenerated NP cells versus mildly degenerated NP cells (Stich et al., 2015). In a study using human synovial stem cells and decellularized extracellular matrix (dECM) substrates derived from human and porcine cells as a preconditioning/expansion strategy, Zhang and colleagues reported significant changes in HLA-DR surface marker expression for porcine dECM preconditioned cells. Interestingly, human-derived dECM did not increase HLA-DR expression in allogeneic stem cells expanded on its surface, but reported a greater than six-fold increase in HLA-DR expression following expansion on porcine dECM versus controls (Zhang et al., 2015). Similarly, Yuan and colleagues reported that microenvironment and scaffolding can modulate HLA-DR expression in chondrocytes (Yuan et al., 2011). A recent study also showed that mesenchymal stem cells seeded 
in various collagen-based scaffolds, hydrogel and sponge, exhibited increases in the production of immunoregulatory factors indoleamine 2,3-dioxygenase (IDO), nitric oxide synthase (NOS), prostaglandin-endoperoxide synthase (PTGS), and hepatocyte growth factor (HGF) compared to 2D culture (Yang et al., 2017), and are paracrine immunomodulators implicated in tissue regeneration (Bassi et al., 2012). Similar results were further confirmed and exemplified in a later study from the same group, where they reported that rabbit chondrocytes seeded on hydrogel-ECM scaffolds could control and lessen adverse immunogenicity of these preconditioned cells (Yuan et al., 2014). Although these studies have individually suggested that the 3D structure, mechanical properties, and composition of 3D scaffolds play roles in the immune-related changes of seeded cells, it remains to be determined in comparative studies which of these properties are predominant influences. 


\section{Detection of immune issues and future directions}

Despite the known potential benefits of various preconditioning strategies for cell culture (Pei 2017), it is imperative that these cells do not elicit immune responses when introduced or reintroduced to a patient. For this reason, more studies are needed to fully evaluate the immunophenotypic changes in preconditioned cells and to perform the necessary experiments to ultimately elucidate the immune-related benefits and caveats of each preconditioning strategy. One of the initial experiments for assaying immune changes should include flow cytometry for immune surface marker analysis following preconditioning, particularly HLA-DR, complemented with Tcell costimulatory markers CD80 and CD86, as well as apoptosis-related markers CD95 (Fas) and CD178 (FasL), which have been implicated in intervertebral disc immunity (Chen et al., 2015; Ma et al., 2015). Furthermore, studies investigating the relationships between preconditioning and Fasmediated apoptosis could uncover new, clinically relevant results for future therapeutic applications in intervertebral disc regeneration. To date, few studies have compared the immunomodulatory properties of various preconditioning methods in a single well-controlled study. One common approach to model potential immune response to preconditioned cells is an MLR assay using labeled immune cells, typically isolated from a blood sample. Several groups have used a version of this technique to test preconditioned cells (Stich et al., 2015; Yuan et al, 2011). Notably, there is a great demand for in vivo studies to investigate the role of preconditioned cells for safe intervertebral disc tissue repair, especially those that can further evaluate the effects of preconditioning-induced changes in immune characteristics. It is reasonably suspected that one preconditioning strategy is unlikely to be most superior or ideal for all tissues in terms of immunomodulation, especially since various tissues are dominated by distinct immunoregulatory factors and different immune cells (Yang et al., 2011). With clinical applications in mind, an ideal 
preconditioning method or combination strategy should consider the multifaceted and specific nature of the immune reactions present in a particular target tissue, as well as the potentially altered immune properties of the preconditioned cell population. 


\section{References}

Bassi ÊJ, de Almeida DC, Moraes-Vieira PM, Câmara NO. Exploring the role of soluble factors associated with immune regulatory properties of mesenchymal stem cells. Stem Cell Rev. 2012 Jun;8(2):329-42. doi: 10.1007/s12015-011-9311-1. Review.

Belykh E, Krutko AV, Baykov ES, Giers MB, Preul MC, Byvaltsev VA. Preoperative estimation of disc herniation recurrence after microdiscectomy: predictive value of a multivariate model based on radiographic parameters. Spine J. 2016 Oct 17. pii: S1529-9430(16)31017-8. doi: 10.1016/j.spinee.2016.10.011. [Epub ahead of print] PubMed PMID: 27765709.

Chen SQ, Lin JP, Zheng QK, Chen SJ, Li M, Lin XZ, Wang SZ. Protective effects of paeoniflorin against FasL-induced apoptosis of intervertebral disc annulus fibrosus cells via Fas-FasL signalling pathway. Exp Ther Med. 2015 Dec;10(6):2351-2355. PubMed PMID: 26668640; PubMed Central PMCID: PMC4665715.

Dighe PA, Viswanathan P, Mruthunjaya AK, Seetharam RN. Effect of bFGF on HLA-DR expression of human bone marrow-derived mesenchymal stem cells. J Stem Cells. 2013;8(1):4357. PubMed PMID: 24459812.

Ellman MB, An HS, Muddasani P, Im HJ. Biological impact of the fibroblast growth factor family on articular cartilage and intervertebral disc homeostasis. Gene. 2008 Aug 15;420(1):82-9. doi: 10.1016/j.gene.2008.04.019. Review. PubMed PMID: 18565695; PubMed Central PMCID: PMC2525607.

Fu Q, Fu TM, Cruz AC, Sengupta P, Thomas SK, Wang S, Siegel RM, Wu H, Chou JJ. Structural Basis and Functional Role of Intramembrane Trimerization of the Fas/CD95 Death Receptor. Mol Cell. 2016 Feb 18;61(4):602-13. doi: 10.1016/j.molcel.2016.01.009. PubMed PMID: 26853147; PubMed Central PMCID: PMC4761300.

Gunzburg R, Parkinson R, Moore R, Cantraine F, Hutton W, Vernon-Roberts B, Fraser R. A cadaveric study comparing discography, magnetic resonance imaging, histology, and mechanical behavior of the human lumbar disc. Spine (Phila Pa 1976). 1992 Apr;17(4):417-26. PubMed

Imai Y, Kimura T, Murakami A, Yajima N, Sakamaki K, Yonehara S. The CED-4-homologous protein FLASH is involved in Fas-mediated activation of caspase-8 during apoptosis. Nature. 1999 Apr 29;398(6730):777-85. Erratum in: Nature 1999 Jul 1;400(6739):89. PubMed PMID: 10235259.

Ito K, Creemers L. Mechanisms of intervertebral disk degeneration/injury and pain: a review. Global Spine J. 2013 Jun;3(3):145-52. doi: 10.1055/s-0033-1347300. Review. PubMed PMID: 24436865 ; PubMed Central PMCID: PMC3854582.

Li X, Wang Y, Zhao Y, Yang H, Tong A, Zhao C, Shi H, Li Y, Wang Z, Wei Y. Immunotherapy of tumor with vaccine based on basic fibroblast growth factor-activated fibroblasts. J Cancer Res Clin Oncol. 2014 Feb;140(2):271-80. doi: 10.1007/s00432-013-1547-5. PubMed PMID: 24322179. 
Li X, An HS, Ellman M, Phillips F, Thonar EJ, Park DK, Udayakumar RK, Im HJ. Action of fibroblast growth factor- 2 on the intervertebral disc. Arthritis Res Ther. 2008;10(2):R48. doi: 10.1186/ar2407. PubMed PMID: 18435858; PubMed Central PMCID: PMC2453768.

Liu X, Luo D, Yang N. Cytosolic Low Molecular Weight FGF2 Orchestrates RIG-I-Mediated Innate Immune Response. J Immunol. 2015 Nov 15;195(10):4943-52. doi: 10.4049/jimmunol.1501503. PubMed PMID: 26466960; PubMed Central PMCID: PMC4637180.

Ma CJ, Liu X, Che L, Liu ZH, Samartzis D, Wang HQ. Stem Cell Therapies for Intervertebral Disc Degeneration: Immune Privilege Reinforcement by Fas/FasL Regulating Machinery. Curr Stem Cell Res Ther. 2015;10(4):285-95. Review. PubMed PMID: 25381758.

Martin I, Vunjak-Novakovic G, Yang J, Langer R, Freed LE. Mammalian chondrocytes expanded in the presence of fibroblast growth factor 2 maintain the ability to differentiate and regenerate three-dimensional cartilaginous tissue. Exp Cell Res. 1999 Dec 15;253(2):681-8. PubMed PMID: 10585291.

Noh KH, Kim SH, Kim JH, Song KH, Lee YH, Kang TH, Han HD, Sood AK, Ng J, Kim K, Sonn $\mathrm{CH}$, Kumar V, Yee C, Lee KM, Kim TW. API5 confers tumoral immune escape through FGF2dependent cell survival pathway. Cancer Res. 2014 Jul 1;74(13):3556-66. doi: 10.1158/00085472.CAN-13-3225. PubMed PMID: 24769442; PubMed Central PMCID: PMC4394897.

Pei M. Environmental preconditioning rejuvenates adult stem cells' proliferation and chondrogenic potential. Biomaterials. 2016 Nov 25;117:10-23. doi: 10.1016/j.biomaterials.2016.11.049. [Epub ahead of print] Review. PubMed PMID: 27923196.

Pizzute T, Zhang Y, Emery SE, Pei M. Preconditioning strategies unequally impact regeneration of nucleus pulposus cells from human herniated discs. Spine 2017 in review

Rohlmann A, Zander T, Schmidt H, Wilke HJ, Bergmann G. Analysis of the influence of disc degeneration on the mechanical behaviour of a lumbar motion segment using the finite element method. J Biomech. 2006;39(13):2484-90. PubMed PMID: 16198356.

Sakai D, Grad S. Advancing the cellular and molecular therapy for intervertebral disc disease. Adv Drug Deliv Rev. 2015 Apr;84:159-71. doi: 10.1016/j.addr.2014.06.009. Review. PubMed PMID: 24993611.

Stich S, Stolk M, Girod PP, Thomé C, Sittinger M, Ringe J, Seifert M, Hegewald AA. Regenerative and immunogenic characteristics of cultured nucleus pulposus cells from human cervical intervertebral discs. PLoS One. 2015 May 19;10(5):e0126954. doi: 10.1371/journal.pone.0126954. PubMed PMID: 25993467; PubMed Central PMCID: PMC4438063.

Sun Z, Liu ZH, Chen YF, Zhang YZ, Wan ZY, Zhang WL, Che L, Liu X, Wang HQ, Luo ZJ. Molecular immunotherapy might shed a light on the treatment strategies for disc degeneration and herniation. Med Hypotheses. 2013 Sep;81(3):477-80. doi: 10.1016/j.mehy.2013.06.014. PubMed PMID: 23849654.

Takada T, Nishida K, Doita M, Kurosaka M. Fas ligand exists on intervertebral disc cells: a potential molecular mechanism for immune privilege of the disc. Spine (Phila Pa 1976). 2002 Jul 15;27(14):1526-30. PubMed PMID: 12131712. 
Vincent T, Hermansson M, Bolton M, Wait R, Saklatvala J. Basic FGF mediates an immediate response of articular cartilage to mechanical injury. Proc Natl Acad Sci U S A. 2002 Jun 11;99(12):8259-64. PubMed PMID: 12034879; PubMed Central PMCID: PMC123055.

Wang J, Tang T, Yang H, Yao X, Chen L, Liu W, Li T. The expression of Fas ligand on normal and stabbed-disc cells in a rabbit model of intervertebral disc degeneration: a possible pathogenesis. J Neurosurg Spine. 2007 May;6(5):425-30. PubMed PMID: 17542508.

Westendorp MO, Frank R, Ochsenbauer C, Stricker K, Dhein J, Walczak H, Debatin KM, Krammer PH. Sensitization of T cells to CD95-mediated apoptosis by HIV-1 Tat and gp120. Nature. 1995 Jun 8;375(6531):497-500. PubMed PMID: 7539892.

Wuertz K, Vo N, Kletsas D, Boos N. Inflammatory and catabolic signalling in intervertebral discs: the roles of NF-кB and MAP kinases. Eur Cell Mater. 2012 Feb 16;23:103-19; discussion 119-20. PubMed PMID: 22354461.

Yang J, Chen X, Yuan T, Yang X, Fan Y, Zhang X. Regulation of the secretion of immunoregulatory factors of mesenchymal stem cells (MSCs) by collagen-based scaffolds during chondrogenesis. Mater Sci Eng C Mater Biol Appl. 2017 Jan 1;70(Pt 2):983-991. doi: 10.1016/j.msec.2016.04.096. PubMed PMID: 27772730.

Yuan T, Luo H, Tan J, Fan H, Zhang X. The effect of stress and tissue fluid microenvironment on allogeneic chondrocytes in vivo and the immunological properties of engineered cartilage. Biomaterials. 2011 Sep;32(26):6017-24. doi: 10.1016/j.biomaterials.2011.04.041. PubMed PMID: 21676457.

Yuan T, Zhang L, Li K, Fan H, Fan Y, Liang J, Zhang X. Collagen hydrogel as an immunomodulatory scaffold in cartilage tissue engineering. J Biomed Mater Res B Appl Biomater. 2014 Feb;102(2):337-44. doi: 10.1002/jbm.b.33011. PubMed PMID: 24000202.

Zhang Y, Pizzute T, Li J, He F, Pei M. sb203580 preconditioning recharges matrix-expanded human adult stem cells for chondrogenesis in an inflammatory environment - A feasible approach for autologous stem cell based osteoarthritic cartilage repair. Biomaterials. 2015 Sep;64:88-97. doi: 10.1016/j.biomaterials.2015.06.038. PubMed PMID: 26122165; PubMed Central PMCID: PMC4503335. 
Figure 1.4.1:

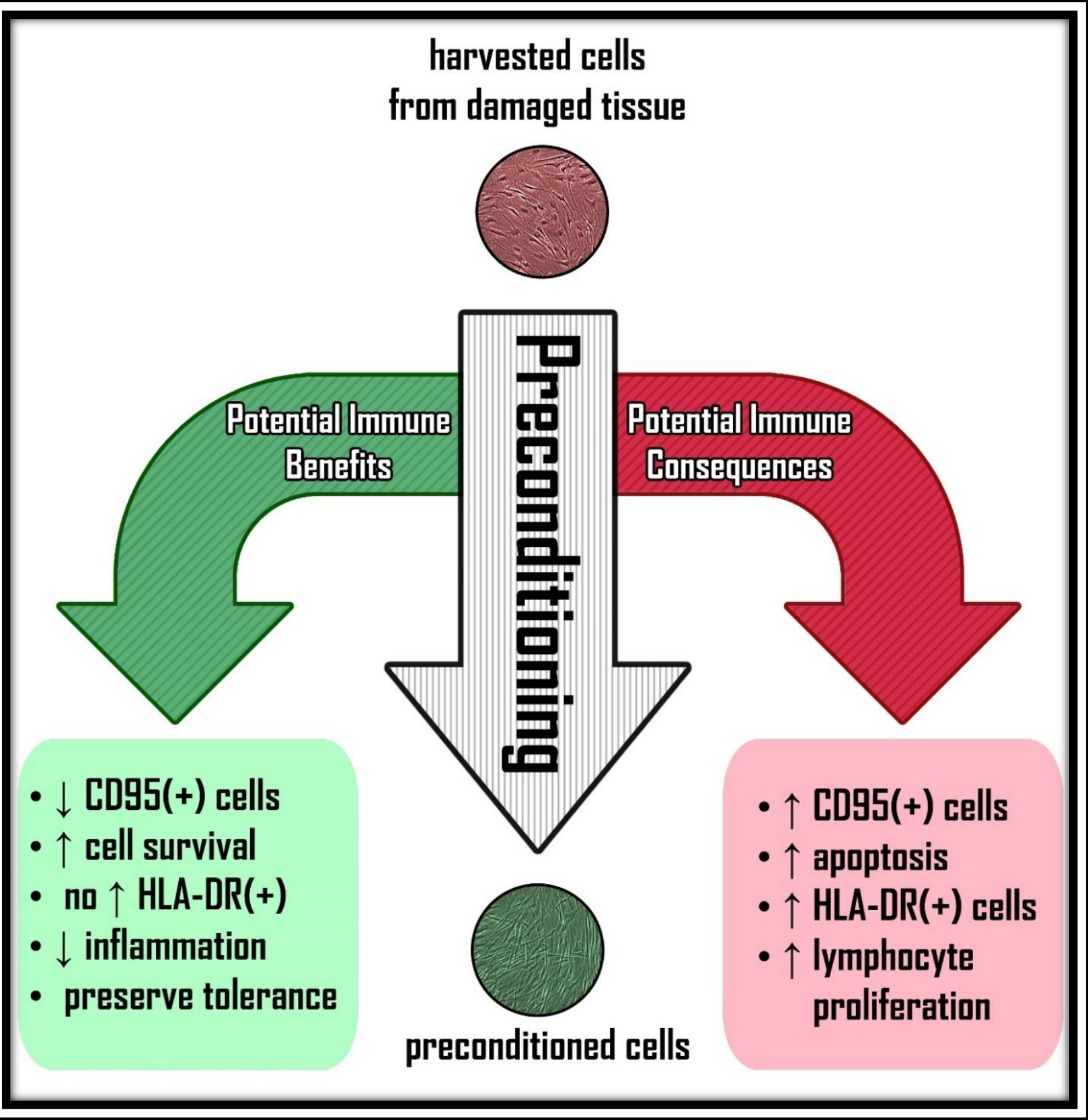


Figure 1.4.1: Cell preconditioning and potential immune effects. Although preconditioning strategies can be effective for cell rejuvenation, they may elicit changes in the preconditioned cells' immunophenotypes or immune-related characteristics. 


\section{Chapter 2:}

\section{Fibroblast Growth Factor Ligand Dependent}

\section{Proliferation and Chondrogenic Differentiation of}

\section{Synovium-Derived Stem Cells and Concomitant}

\section{Adaptation of Wnt/Mitogen-Activated Protein Kinase}

\section{Signals}

As published in Tissue Engineering: Part A; Aug 2016

Tyler T. Pizzute, Jingting Li, Ying Zhang, Mary E. Davis, Ming Pei.

Stem Cell and Tissue Engineering Laboratory, Department of Orthopaedics, West Virginia University, One Medical Center Drive, PO Box 9196, Morgantown, WV 26506-9196, USA.

Running Title: FGF Ligand and Stem Cell Differentiation

Corresponding author: Ming Pei, MD, $\mathrm{PhD}$

Stem Cell and Tissue Engineering Laboratory, Department of Orthopaedics, West Virginia University, PO Box 9196, One Medical Center Drive, Morgantown, WV 26506-9196, USA, Telephone: 304-293-1072; Fax: 304-293-7070; Email: mpei@hsc.wvu.edu 


\begin{abstract}
Cell expansion techniques commonly utilize exogenous factors to increase cell proliferation and create a larger cell population for use in cell-based therapies. One strategy for cartilage regenerative therapies is autologous stem cell expansion and fibroblast growth factor (FGF) supplementation during cell expansion, particularly FGF-2. However, it is unknown whether FGF-10, another FGF implicated in limb and skeletal development, can elicit the same rejuvenation responses in terms of proliferation and differentiation of human synoviumderived stem cells (SDSCs). In this study, we expanded SDSCs in either FGF-2 or FGF-10 for 7 days; a control group had no treatment. FGF-2 and FGF-10 supplementation was also exclusively tested during the differentiation phase. Expanded SDSCs were evaluated for their ability to successfully engage in chondrogenic and osteogenic differentiation. We found that FGF-2 supplementation during proliferation, but not differentiation, was able to increase glycosaminoglycan deposition, pellet size, and chondrogenic gene expression following chondrogenic induction, as well as increased calcium deposition, alkaline phosphatase activity, and expression of vital osteogenic differentiation genes following osteogenic induction. FGF-10 did not elicit a similar preconditioning effect. We also observed changes of both Wnt signals and mitogen-activated protein kinase expression during SDSC chondrogenesis, which occurred in a manner dependent upon the supplementation phase of FGF-2 administration. These results indicated that FGF-2, but not FGF-10, may be supplemented during stem cell expansion to prime cells for successful chondrogenesis and osteogenesis.
\end{abstract}




\section{Introduction}

Adult Mesenchymal Stem Cells (MSCs) are promising alternative cell sources for cartilage tissue engineering due to the shortage of autologous chondrocytes for cell-based regenerative therapies. ${ }^{1}$ However, adult stem cells obtained from tissues have either less chondrogenic potential despite large amounts such as adipose stem cells or higher endochondral ossification and limited sample size such as bone marrow stromal cells (BMSCs). $\stackrel{2}{ }$ One promising MSC population found in the joint, known as synovium-derived stem cells (SDSCs), has recently been characterized as tissue-specific stem cells for chondrogenesis. ${ }^{3}$ Strategies that allow for MSC proliferation may be necessary to achieve a substantial and usable cell number for therapies; however, the initial MSC numbers are low and their differentiation potential can be compromised following excessive ex vivo expansion, with notable increases in cell senescence marker expression and decreased proliferative capacity. ${ }^{4}$ This situation can be further complicated when combined with other preexisting and potentially detrimental factors such as donor age and disease pathology. $\underline{5}$

It is known that the fibroblast growth factor (FGF) family is involved in limb and joint development, as well as various stages of skeletal and cartilage formation and maturation. ${ }^{6}$ For instance, FGF-2 is involved in early cartilage development and can cause dramatic increases in cell proliferation in chondrocytes and osteoblasts. ${ }^{7}$ In addition, another member of the FGF family, FGF-10, is known to be vital to limb bud initiation and development,, 9 but less is known about the ability of FGF-10 to modulate chondrogenic activity, although a recent report indicated that FGF-

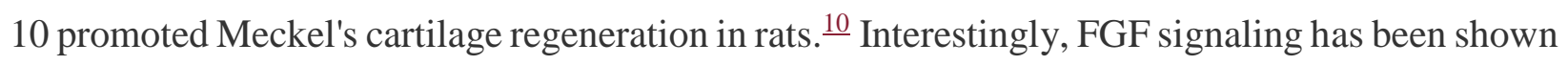
to maintain MSCs in an undifferentiated state during proliferation while preserving their multipotentiality,, 11 which further establishes the FGF family's potential benefits for preconditioning strategies. 
Our recent findings suggest that decellularized extracellular matrix (dECM) deposited by stem cells could also provide a preconditioning strategy that stem cells could be greatly expanded with enhanced chondrogenic potential ${ }^{12}$ or endochondral ossification. ${ }^{\frac{13}{3}}$ Interestingly, microarray data from these dECM studies have shown that, among all FGFs, FGF-2 and FGF-10 are the most significantly regulated factors in human SDSCs following their expansion on dECM (Fig. 1). It is unknown whether a similar rejuvenating effect can be achieved on human SDSC chondrogenesis and osteogenesis through FGF-2 and FGF-10 supplementation in the cell expansion phase, as well as supplementation in the differentiation phase. Due to the importance and potential impacts in stem cell-mediated chondrogenesis, $\stackrel{14,15}{\underline{1}}$ the content of both Wnt and mitogen-activated protein kinase (MAPK) activation, which have been demonstrated in our earlier dECM preconditioning study, $\frac{16}{}$ will also be assessed in this study following FGF ligand rejuvenation. 


\section{Materials and Methods}

Evaluation of cell proliferation, surface phenotypes, and differentiation gene

\section{Cell culture and proliferation}

Human adult SDSCs obtained from Asterand (North America Laboratories, Detroit, MI) were cultured in growth medium containing alpha-minimum essential medium, $10 \%$ fetal bovine serum, $100 \mathrm{U} / \mathrm{mL}$ penicillin, $100 \mu \mathrm{g} / \mathrm{mL}$ streptomycin, and $0.25 \mu \mathrm{g} / \mathrm{mL}$ fungizone as described previously.16-18 Passage 4 SDSCs were treated with 10 ng/mL of FGF-2 or FGF-10 (PeproTech, Inc., Rocky Hill, NJ) during cell expansion/proliferation (F2P and F10P, respectively) or differentiation (F2D and F10D, respectively) (Fig. 2A). Cells cultured with no FGF treatment acted as a control (CNTL). Cell number was counted in $175 \mathrm{~cm} 2$ flasks $(n=6)$ using a hemocytometer. To determine proliferation index, before cell expansion, SDSCs were labeled with CellVue® Claret at $2 \times 10-6 \mathrm{M}$ for $5 \mathrm{~min}$ according to the manufacturer's protocol (Sigma-Aldrich, St. Louis, MO). After 6 days of proliferation, expanded cells were collected and measured using a BD FACSCalibur ${ }^{\mathrm{TM}}$ flow cytometer (dual laser) (BD Biosciences, San Jose, CA). Twenty thousand events of each sample were collected using CellQuest Pro software (BD Biosciences), and cell proliferation index was analyzed by ModFit LT ${ }^{\mathrm{TM}}$ version 3.1 (Verity Software House, Topsham, ME).

\section{Surface phenotypes of expanded cells}

The following primary antibodies were used in flow cytometry analysis to detect expanded SDSC surface immunophenotype profiles: CD29 (Abcam, Cambridge, MA), CD90 (BD Pharmingen, 
San Jose, CA), CD105 (BioLegend, San Diego, CA), the stage-specific embryonic antigen 4 (SSEA4; BioLegend), and isotype-matched immunoglobulin Gs (IgGs; Beckman Coulter, Fullerton, CA). The secondary antibody was goat anti-mouse $\operatorname{IgG}(\mathrm{H}+\mathrm{L})$ R-phycoerythrin conjugated (Life Technologies, Carlsbad, CA). Samples $(n=3)$ of each $2 \times 105$ expanded cells were incubated on ice in cold phosphate-buffered saline containing $0.1 \%$ ChromPure Human IgG whole molecule (Jackson ImmunoResearch Laboratories, West Grove, PA) and 1\% NaN3 (SigmaAldrich) for $30 \mathrm{~min}$. The cells were then sequentially incubated in the dark in the primary and secondary antibodies for $30 \mathrm{~min}$. Fluorescence was analyzed by a FACSCalibur (BD Biosciences) using FCS Express 4 software package (De Novo Software, Los Angeles, CA).

\section{Senescence and differentiation gene expression}

Expanded cells were evaluated using real-time polymerase chain reaction (PCR) for senescence and differentiation-related gene changes. Total RNA was extracted from representative samples $(\mathrm{n}=4)$ using an RNase-free pestle in TRIzol ${ }^{\circledR}$ (Life Technologies). Two micrograms of mRNA were used for reverse transcriptase with a High-Capacity cDNA Archive Kit (Applied Biosystems, Foster City, CA) at $37^{\circ} \mathrm{C}$ for $120 \mathrm{~min}$. Senescence-related genes (cyclin-dependent kinase inhibitor 2A [P16; Assay ID Hs00923894_m1], cyclin-dependent kinase inhibitor 1A [P21; Assay ID Hs00355782_m1], and tumor protein p53 [TP53; Assay ID Hs01034249_m1]), chondrogenic marker genes (SRY [sex determining region Y]-box 9 [SOX9; Assay ID Hs00165814_m1], aggrecan [ACAN; Assay ID AIQJAP5], and type II collagen [COL2A1; Assay ID Hs00156568_m1]), and osteogenic genes (Runt-related transcription factor 2 [RUNX2; Assay ID Hs00231692_m1]; Osterix [SP7; Assay ID Hs01866874_s1]; Secreted Phosphoprotein 1 [SPP1; Assay ID hs00959010_m1]; Bone sialoprotein [IBSP; Assay ID hs00173720_m1]; and Bone Gamma-Carboxyglutamate [Gla] Protein [BGLAP; Assay ID Hs01587814_g1]) were customized 
by Applied Biosystems as part of their Custom TaqMan® Gene Expression Assays. Glyceraldehyde-3-Phosphate Dehydrogenase (GAPDH; Assay ID Hs02758991_g1) was carried out as the endogenous control gene. Real-time PCR was performed with the $\mathrm{iCycler} \mathrm{iQ}^{\mathrm{TM}}$ Multicolor RT-PCR Detection and calculated by computer software (PerkinElmer, Wellesley, MA). Relative transcript levels were calculated as $\chi=2-\Delta \Delta \mathrm{Ct}$, in which $\Delta \Delta \mathrm{Ct}=\Delta \mathrm{E}-\Delta \mathrm{C}$, $\Delta \mathrm{E}=\mathrm{Ctexp}-\mathrm{CtGAPDH}$, and $\Delta \mathrm{C}=\mathrm{Ctct} 1-\mathrm{CtGAPDH}$.

Chondrogenic induction and evaluation

\section{Chondrogenic induction}

Expanded cells $(3.0 \times 105)$ were centrifuged at $500 \mathrm{~g}$ for $5 \mathrm{~min}$ in a $15-\mathrm{mL}$ polypropylene tube to form a pellet. After overnight incubation (day 0), the pellets were cultured in a serum-free chondrogenic medium consisting of high-glucose Dulbecco's modified Eagle's medium, $40 \mu \mathrm{g} / \mathrm{mL}$ proline, $100 \mathrm{nM}$ dexamethasone, $100 \mathrm{U} / \mathrm{mL}$ penicillin, $100 \mu \mathrm{g} / \mathrm{mL}$ streptomycin, $0.1 \mathrm{mM}$ ascorbic acid-2-phosphate, and $1 \times$ ITS $^{\text {TM }}$ Premix (BD Biosciences) with the supplementation of $10 \mathrm{ng} / \mathrm{mL}$ transforming growth factor beta3 (TGF- $\beta 3$, PeproTech, Inc.) in a 5\% O2 incubator as long as 21 days. F2D and F10D groups were also supplemented with $10 \mathrm{ng} / \mathrm{mL}$ FGF-2 or FGF-10, respectively. The pellets were evaluated using real-time PCR for chondrogenic marker genes (ACAN and COL2A1), type I collagen (COL1A1; Assay ID Hs00164004_m1), hypertrophic genes (type X collagen [COLXA1; Assay ID Hs00166657_m1] and alkaline phosphatase [ALP; Assay ID Hs01029144_m1]), histology and immunohistochemistry (IHC) for staining, and biochemical analysis for both DNA and glycosaminoglycan (GAG) amounts. 


\section{Histology and immunostaining}

Representative pellets $(n=2)$ were fixed in $4 \%$ paraformaldehyde at $4^{\circ} \mathrm{C}$ overnight, followed by dehydrating in a gradient ethanol series, clearing with xylene, and embedding in paraffin blocks. Five-micrometer thick sections were stained with Alcian blue (counterstained with fast red) for sulfated GAGs. For IHC, the sections were immunolabeled with primary antibody against type II collagen (Col2; II-II6B3, Developmental Studies Hybridoma Bank, Iowa City, IA], followed by the secondary antibody of biotinylated horse anti-mouse IgG (Vector, Burlingame, CA). Immunoactivity was detected using VECTASTAIN ABC reagent (Vector) with 3, 3'diaminobenzidine as a substrate.

\section{Biochemical analysis for DNA and GAG contents}

Representative pellets $(n=4)$ were digested at $60^{\circ} \mathrm{C}$ for $4 \mathrm{~h}$ with $125 \mu \mathrm{g} / \mathrm{mL}$ papain in PBE buffer (100 mM phosphate, $10 \mathrm{mM}$ ethylenediaminetetraacetic acid, $\mathrm{pH} 6.5$ ) containing $10 \mathrm{mM}$ cysteine. To quantify cell density, the amount of DNA in the papain digestion was measured using the Quant-iT ${ }^{\mathrm{TM}}$ PicoGreen ${ }^{\circledR}$ dsDNA Assay Kit (Life Technologies) with a CytoFluor ${ }^{\circledR}$ Series 4000

(Applied Biosystems). GAG was measured using dimethylmethylene blue dye and a Spectronic ${ }^{\text {TM }}$ BioMate $^{\mathrm{TM}} 3$ Spectrophotometer (Thermo Fisher Scientific, Waltham, MA) with bovine chondroitin sulfate (Sigma-Aldrich) as a standard.

Expression of both Wnt and MAPK signals following FGF-mediated chondrogenesis

Expanded cells and subsequent pellets were dissolved in the lysis buffer (Cell Signaling, Danvers, MA) with protease inhibitors. Total proteins were quantified using BCA ${ }^{\mathrm{TM}}$ Protein Assay Kit (Thermo Fisher Scientific). Thirty micrograms of protein from each sample were denatured and 
separated using NuPAGE® Novex ${ }^{\circledR}$ Bis-Tris Mini Gels in the XCell SureLock ${ }^{\mathrm{TM}}$ Mini-Cell (Life Technologies) at $120 \mathrm{~V}$ at $4^{\circ} \mathrm{C}$ for $3 \mathrm{~h}$. Bands were transferred onto a nitrocellulose membrane using an XCell $\mathrm{II}^{\mathrm{TM}}$ Blot module (Life Technologies) at $15 \mathrm{~V}$ at $4^{\circ} \mathrm{C}$ overnight. The membrane was incubated with primary monoclonal antibodies in 5\% bovine serum albumin in TBST buffer (10 mM Tris-HCl, $\mathrm{pH} 7.5,150 \mathrm{mM} \mathrm{NaCl}, 0.05 \%$ TWEEN 20$)$ for $1 \mathrm{~h}$ ( $\beta$-actin served as an internal control), followed by the secondary antibody of horseradish peroxidase-conjugated goat antimouse (Thermo Fisher Scientific) for $1 \mathrm{~h}$. SuperSignal West Femto Maximum Sensitivity Substrate and CL-XPosure Film (Thermo Fisher Scientific) were used for exposure. The primary antibodies used in immunoblotting included the MAPK Family Antibody Sampler Kit (extracellular signal-regulated protein kinases 1 and 2 [Erk1/2], Jun N-terminal kinase [Jnk], and p38), phosphorylated (p-) MAPK Family Antibody Sampler Kit, and Wnt Signaling Antibody Sampler Kit (Cell Signaling). Wnt11 polyclonal antibody was obtained from Thermo Fisher

Scientific. Wnt signals were also evaluated using real-time PCR (WNT3A; Assay ID Hs00263977_m1, WNT5A; Assay ID Hs00998537_m1, and WNT11; Assay ID Hs00182986_m1) following cell expansion and chondrogenic differentiation.

\section{Osteogenic induction and evaluation}

Expanded cells $(\mathrm{n}=3)$ cultured for 21 days in osteogenic medium (growth medium supplemented with $0.1 \mu \mathrm{M}$ dexamethasone, $10 \mathrm{mM} \beta$-glycerol phosphate, $50 \mu \mathrm{M}$ ascorbate-2-phosphate, and $0.01 \mu \mathrm{M}$ 1,25-dihydroxyvitamin D3) were collected for ALP activity assay with a reagent kit (Sigma-Aldrich) by measuring the formation of p-Nitrophenol from p-nitrophenyl phosphate following the manufacturer's instructions. For evaluation of calcium deposition, induced cells $(\mathrm{n}=3)$ were fixed with $70 \%$ ice-cold ethanol for $1 \mathrm{~h}$ and then incubated in $40 \mathrm{mM}$ Alizarin Red $\mathrm{S}$ (ARS) at $\mathrm{pH} 4.2$ for $20 \mathrm{~min}$ with agitation. After rinsing, matrix mineral-bound staining was 
photographed. Quantification of staining was performed by staining density using ImageJ software.

\section{Statistical analyses}

Analysis of variance with pairwise comparison and t-test was used to compare measurements between different groups. All statistical analyses were performed with SPSS 13.0 statistical software (SPSS, Inc., Chicago, IL). p-Values $<0.05$ were considered statistically significant. 


\section{Results}

Addition of FGF-2 but not FGF-10 during the expansion phase promoted SDSC proliferation

After 7 days of monolayer expansion, SDSCs without FGF treatment exhibited enlarged cell morphology which, in the presence of FGF-2, became notably smaller and fibroblast-like shaped cells with a glistening outline. Both proliferation index (Fig. 2B) and cell number counting data (Fig. 2C) suggested that FGF-2 significantly enhanced SDSC proliferation, while FGF-10 supplementation did not induce greater cell proliferation compared to the control group. This result is also supported by SSEA4 expression levels evaluated by flow cytometry (Fig. 2D). Surprisingly, other stem cell markers (Fig. 2D), including CD29, CD90, and CD105, exhibited decreases in median fluorescence intensity when SDSCs were expanded in the presence of FGF2. Similar to the cell proliferation data, FGF-10 treatment did not elicit any meaningful differences in the expression of stem cell markers.

Addition of FGF-2 led to changes in expression of senescence and differentiation-related genes

TaqMan® real-time PCR data showed that FGF-2 treatment upregulated P16 expression level in expanded cells compared with the control group but downregulated senescent genes P21 and TP53 expression levels (Fig. 3A). We also found that FGF-2 treatment significantly increased transcriptional factor SOX9, and FGF-10 treatment decreased SOX9 expression. This result was also accompanied by early and dramatic decreases in chondrogenic marker gene expression (ACAN and COL2A1) (Fig. 3B). For osteogenic genes, FGF-10 preconditioning significantly decreased RUNX2 expression, while FGF-2 produced similar RUNX2 expression levels as the control. Interestingly, FGF-2 supplementation, but not FGF-10, led to significant increases in SP7, SPP1, IBSP, and BGLAP gene expression versus other groups (Fig. 3C). 
Addition of FGF-2 but not FGF-10 during the expansion phase promoted SDSC chondrogenic potential

After 21-day chondrogenic induction, FGF-2 pretreated SDSCs yielded pellets with the largest size and most intense staining for sulfated GAGs by Alcian blue and Col2 by IHC compared to the other four groups, which exhibited similar pellet sizes and staining intensities (Fig. 4A). These findings were confirmed by biochemical analysis data in which the FGF-2 pretreatment group yielded the highest DNA ratio by day 0, GAG amount per pellet, and ratio of GAG to DNA, known as the chondrogenic index (Fig. 4B). TaqMan real-time PCR data showed that, after 21-day chondrogenic induction, FGF-2 pretreated cells yielded the lowest expression of COL1A1, but the highest levels of COL2A1 and ACAN, while FGF-2 treated cell pellets yielded the second lowest level of COL1A1 and the lowest levels of COL2A1 and ACAN. Both FGF-10 treatment groups, regardless of supplementation phase, yielded similar expression of COL1A1 and ACAN, which was lower than the control group (Fig. 4C), but still significantly greater than either FGF-2 treatment group. We also found that FGF-2 pretreatment yielded cells with the highest level of COL10A1 and ALP, while FGF-2 treatment yielded cells with the lowest level of ALP mRNA (Fig. 4D).

Wnt and MAPK signals following FGF-2 preconditioning and chondrogenic differentiation

TaqMan real-time PCR data showed that FGF-2 pretreated SDSCs displayed a significant upregulation of WNT5A and WNT3A and downregulation of WNT11 (Fig. 5A), which was confirmed by Western blot data (Fig. 5B); compared to significant upregulation of both p-p38 and p-Jnk signals in FGF-2 pretreated SDSCs, total Erk1/2 was markedly reduced with a modest suppression in p-Erk (Fig. 5C). Following chondrogenic differentiation, FGF-2 pretreated 
SDSCs showed decreased levels of Wnt5a, but increased levels of Wnt11; however, FGF-2 treatment in chondrogenic induction exhibited an opposite trend (Fig. 5D, E). Interestingly, FGF2 pretreated SDSCs also showed decreased levels of p38, Jnk, and Erk1/2 compared to other groups (Fig. 5F).

Effect of FGF-2 and FGF-10 on SDSC osteogenic differentiation

After 21-day osteogenic induction, FGF-2 pretreated SDSCs exhibited the highest density of both ARS (Fig. 6A) and ALP staining (Fig. 6B), indicating stronger calcium deposition and ALP expression, respectively. Interestingly, FGF-2 treatment in osteogenic induction significantly decreased ARS staining compared to other groups, while FGF-10 treatment either during cell expansion or osteogenic induction had no evident effect on osteogenic differentiation of expanded SDSCs (Fig. 6C, D). 


\section{Discussion}

In this study, we sought to determine whether FGF-2 or FGF-10 supplementation during the proliferation phase or the differentiation phase could improve human SDSC chondrogenesis and osteogenesis. In addition, we wanted to determine if both Wnt and MAPK signals were involved in FGF ligand-mediated SDSC proliferation and lineage differentiation. The present study suggested that FGF-2 supplementation during the proliferation phase can precondition SDSCs to undergo more successful chondrogenesis and osteogenesis following differentiation induction, which was superior to FGF-10 supplementation, no treatment, and the addition of either FGF during differentiation. The early upregulation of Wnt3a and Wnt5a and early downregulation of Wnt11 are likely to be influential mechanisms involved in FGF preconditioning and successful differentiation along the chondrogenic and osteogenic lineages. Chondrogenic differentiation was complimented by an upregulation of $S O X 9$, a known transcription factor for chondrogenic activity, with FGF-2 supplementation during proliferation. Interestingly, FGF-2 preconditioning during the proliferation phase led to a $R U N X 2$-independent mRNA upregulation of several vital osteogenic genes and more successful osteogenesis in vitro than all other groups. These findings suggested that FGF-2 supplementation during the early proliferation phase can prime human SDSCs for successful chondrogenic and osteogenic differentiation, which may be, in part, modulated through Wnt and MAPK signaling, but not when supplemented with FGF-2 during differentiation or with FGF-10 at any stage.

Unlike human germ cells, most human somatic cells do not express telomerase and, therefore, lose telomeric DNA during each round of DNA replication. $\frac{19}{}$ Stress conditions could cause cell senescence ${ }^{20}$ resulting from DNA damage triggered by telomere shortening through the p53mediated signaling pathway; on the contrary, p53 inactivation prolongs the lifespan of human 
fibroblasts. ${ }^{21}$ The expression of p21, a p53 target gene, increases in senescent cells. ${ }^{22}$ Since targeted deletion of the $\mathrm{p} 21$ gene is sufficient to escape senescence in human fibroblasts, $\frac{23}{}$ p21 may have a major role in the induction of cellular senescence in human fibroblasts by inhibiting the activity of cyclin-dependent kinases. In this study, preconditioning using FGF-2 dramatically decreased $P 21$ and TP53, indicating a rescue of telomere-dependent intrinsic senescence. Interestingly, both FGF-2 and FGF-10 pretreatment dramatically increased P16 expression in expanded cells, which is usually raised through extrinsic senescence such as reactive oxygen,,$\underline{\underline{ }}$ suggesting that the rescue dominated by FGF-2 pretreatment was independent of the $\mathrm{p} 16 / \mathrm{pRb}$ pathway. Future studies will be necessary to fully validate these trends, determine whether these changes are consistent over several cell passages, and discover a possible mechanism in which FGF-2 may be regulating cell senescence.

In this study, we found that FGF-2 rather than FGF-10 promoted SDSC proliferation. The potential underlying mechanisms could be selection of a particular subset of cells by telomere length in FGF-2-expanded MSCs. $.24,25$ Despite the role in regulating the proliferation and maintenance of

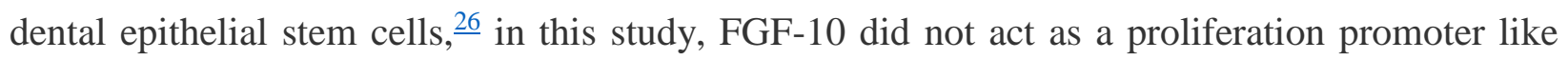
FGF-2. Interestingly, FGF-2 pretreatment decreased CD29, CD90, and CD105 expression, but increased SSEA4. This finding is consistent with a report by Hagmann et al. in which they found that CD90, CD105, and CD146 were significantly decreased in human BMSCs after treatment with FGF-2. ${ }^{27}$ One potential explanation is that FGF-2 could decrease the expression of TGF- $\beta$, which is a promoter of cell senescence. ${ }^{28}$ Sacchetti et al. found that FGF-2 pretreatment decreased the expression of CD105 (endoglin), a TGF- $\beta$ coreceptor. ${ }^{29}$ Furthermore, Ito et al. found that downregulation of TGF- $\beta$ signaling was responsible for the rejuvenating effect of FGF-2 pretreatment in terms of osteogenic and chondrogenic potential in human MSCs. $\frac{30}{} \mathrm{~A}$ similar trend 
in surface phenotypes was observed in human SDSCs after expansion on dECM deposited by stem cells. $\frac{17,18}{}$ Like pretreatment with FGF-2, this dECM expansion approach also plays a role in the preconditioning of expanded stem cells in both proliferation and differentiation capacity. $\frac{31}{}$ Since CD29, CD90, and CD105 are considered traditional surface markers for stem cells,,$\underline{32}$ the decrease in these markers during stem cell rejuvenation raises questions regarding their defined roles as they pertain to stem cell "stemness."

Despite the fact that both chondrogenic and osteogenic potentials were significantly increased after FGF-2 pretreatment, we did find a discrepancy in the expression of transcriptional and differentiation genes for a specific lineage in expanded cells. In terms of osteogenesis, FGF-2 pretreated SDSCs exhibited an upregulation of both transcriptional gene (SP7) and matrix genes (SPP1, IBSP, and BGLAP), despite no significant change in another transcriptional gene (RUNX2). This finding might be explained by negative feedback modulation through addition of exogenous FGF-2, which can lower endogenous FGF-2 expression, $\underline{33}$ resulting in a decrease of the osteogenesis inhibitory effect exerted by FGF-2 during subsequent osteogenic induction.,$\frac{34, \underline{35}}{\mathrm{In}}$ terms of chondrogenesis, however, FGF-2 pretreated cells exhibited an upregulation of a transcriptional gene (SOX9), but downregulation of matrix genes (ACAN and COL2A1). This finding could be explained by a recent report in which pretreatment with FGF-2 could significantly enhance reprogramming efficiency by downregulating matrix genes such as collagen. $\frac{36}{6}$

Due to the importance of both Wnt and MAPK signaling in cartilage regeneration, $\frac{15}{}$ the signals associated with these two pathways were characterized during FGF-2 pretreatment and subsequent chondrogenic induction. Following FGF-2 supplementation during the proliferative phase, we found that WNT3A and WNT5A mRNAs were markedly increased, while WNT11 mRNA was decreased. Interestingly, a contrasting trend, with WNT5A mRNA expression significantly 
decreased and WNT11 mRNA significantly increased versus other groups, occurred following 21day pellet differentiation culture. This result appears to support the notion that Wnt signals are stage-specifically expressed during chondrogenesis. $\frac{37}{}$ For instance, Wnt5a has been previously implicated as an early promoter $\frac{38}{-}$ and Wnt11, which has been shown to stimulate Col2 deposition,,$^{39}$ is a late promoter of chondrogenesis. ${ }^{40}$ Another study reported that Wnt3a-canonical and noncanonical Wnt pathways counteracted one another in MSC chondrogenesis. ${ }^{41}$ This notion seems well supported by other studies, which suggest that Wnt3a can prevent or inhibit chondrogenesis, while Wnt5a supports cartilage formation, but can impede Col2 expression and induce dedifferentiation. ${ }^{39,42,43}$ These findings support the Wnt expression trends observed in our data, where both Wnt3a and Wnt5a were expressed in earlier stages following proliferation and FGF-2 supplementation but, following chondrogenic differentiation, Wnt3a and Wnt5a mRNAs and proteins were either significantly decreased or undetected. This expression is likely important in maintaining the "stemness" of the SDSCs, which may promote successful responses to chondrogenic media, as well as later expression of Col2, aggrecan, and greater GAG deposition. In addition, it is worth noting that preconditioning methods, which directly or indirectly regulate Wnt signaling, could hold great potential; however, a study by Hoang ${ }^{44}$ raises a valid point that therapies targeting Wnt may have significant side effects due to their defined roles in tissue regeneration and stem cell self-renewal, as well as osteosarcoma. Although aberrant Wnt signaling does not seem to be a concern in this study, specifically with FGF-2 treatment of SDSCs, as evidenced by the reversed trend in the Wnt real-time PCR results from the expansion phase to the differentiation phase, it is a caveat which should be noted when considering therapies which modify Wnt signaling. 
Interestingly, RUNX2 was not significantly increased in either of the FGF supplemented groups versus the control in expanded cells before osteogenic induction. Furthermore, Western blotting revealed that expanded cells treated with FGF-2 during proliferation had robust levels of p-p38 and p-Jnk, but little p-Erk1/2 or Erk1/2. It is known that RUNX2, a transcriptional regulator of osteogenesis, is heavily regulated through the Erk signaling pathway ${ }^{45,46}$; however, it may not be required for osteogenic differentiation to occur. A study using rat BMSCs reported results demonstrating that inhibition of p38 activity alone did not interfere with osteogenesis and suggested that mechanical strain-induced osteogenic differentiation was a result of Erk1/2 and RUNX2 activation. ${ }^{47}$ Additional studies have reported that the inhibition of Erk1/2 and Jnk, but not $\mathrm{p} 38$, has resulted in inhibited osteogenic differentiation ${ }^{48}$; also, decreased RUNX2 expression was observed in BMSCs with the inhibition of Erk1/2 phosphorylation. $\frac{46}{\text { Furthermore, it has been }}$ confirmed that RUNX2 activation occurs through the Erk pathway. ${ }^{45}$ Although p38 is undeniably implicated in osteogenesis, studies targeting the Erk and Jnk pathways through inhibition seem to suggest that the Erk pathway may be more closely related to RUNX2 activation and regulation. Given the growing number of studies reporting RUNX2 activation without p38 signaling, the lack of RUNX2 expression could very likely be directly related to Erk1/2 signaling, which was significantly decreased in our current study compared to robust levels of p-p38 and p-Jnk in the FGF-2 pretreatment group following cell expansion, as evidenced by Western blotting. It is very possible that increases in ALP and ARS staining following successful osteogenic induction in the FGF-2 pretreatment group are the result of increased gene expression of other key osteogenic genes such as $S P 7, S P P 1, I B S P$, and $B G L A P$, which were all significantly increased in the FGF-2 pretreatment group versus control and FGF-10 pretreatment groups, but not through mechanisms related to RUNX2 or Erk1/2 activation. Overall, it seems that the strong ALP activity, calcium 
deposition, and upregulation of several key osteogenic genes in the FGF-2 pretreatment group were primarily driven through $R U N X 2$-independent and Erk1/2-independent signaling and likely occurred through p38, Jnk, and potentially other signaling mechanisms. More studies need to be performed to fully assess the necessity of Erk1/2 and RUNX2 roles in SDSC osteogenesis.

In conclusion, FGF-2 preconditioning led to superior cell proliferation, chondrogenesis, osteogenesis, and overall rejuvenation versus no treatment or FGF-10 preconditioning. Cells preconditioned with FGF-2 during the proliferative phase led to robust chondrogenic pellet formation, accompanied by significant GAG deposition and upregulation of vital chondrogenic gene expression. Likewise, FGF-2 preconditioning produced unmatched osteogenesis, leading to cells with increased gene expression following proliferation, and led to significant increases in ARS and ALP staining for the differentiated cell cultures versus other groups. FGF-2, but not FGF10, preconditioning was able to elicit significant changes in Wnt signal expression, as well as pronounced p38 signaling. FGF-2 seems to be an optimal choice as a preconditioning stimulus for human SDSC chondrogenesis and osteogenesis. 


\section{Acknowledgments}

The authors thank Suzanne Danley for editing the article. This project was partially supported by

Research Grants from the Musculoskeletal Transplant Foundation (MTF) and the National

Institutes of Health (AR062763-01A1 and P20GM103434). 


\section{Disclosure Statement}

No competing financial interests exist. 


\section{References}

1. J. Karnes, Y. Zhang, and M. Pei Cell therapy for the creation of cartilage and related clinical trials. In: N.S. Templeton, ed. Gene and Cell Therapy: Therapeutic Mechanisms and Strategies. 4th edition. Boca Raton, FL: Taylor \& Francis/CRC Press, 2014, pp. 1123-1135.

2. T. Pizzute, K. Lynch, and M. Pei Impact of tissue-specific stem cells on lineage-specific differentiation: a focus on the musculoskeletal system. Stem Cell Rev 11, 119, 2015.

3. B.A. Jones, and M. Pei Synovium-derived stem cells: a tissue-specific stem cell for cartilage engineering and regeneration. Tissue Eng Part B 18, 301, 2012.

4. J.T. Li, and M. Pei Cell senescence: a challenge in cartilage engineering and regeneration. Tissue Eng Part B 18, 270, 2012.

5. K. Lynch, and M. Pei Age associated communication between cells and matrix: a potential impact on stem cell-based tissue regeneration strategies. Organogenesis 10, 289, 2014.

6. D.M. Ornitz, and P.J. Marie Fibroblast growth factor signaling in skeletal development and disease. Genes Dev 29, 1463, 2015.

7. D.M. Ornitz FGF signaling in the developing endochondral skeleton. Cytokine Growth Factor Rev 16, 205, 2005.

8. H. Ohuchi, T. Nakagawa, A. Yamamoto, A. Araga, T. Ohata, Y. Ishimaru, et al. The mesenchymal factor, FGF10, initiates and maintains the outgrowth of the chick limb bud through interaction with FGF8, an apical ectodermal factor. Development 124, 2235, 1997. 
9. K. Sekine, H. Ohuchi, M. Fujiwara, M. Yamasaki, T. Yoshizawa, T. Sato, et al. FGF-10 is essential for limb and lung formation. Nat Genet 21, 138, 1999.

10. F. Terao, I. Takahashi, H. Mitani, N. Haruyama, Y. Sasano, O. Suzuki, et al. Fibroblast growth factor 10 regulates Meckel's cartilage formation during early mandibular morphogenesis in rats. Dev Biol 350, 337, 2011.

11. D.L. Coutu, and J. Galipeau Roles of FGF signaling in stem cell self-renewal, senescence and aging. Aging (Albany NY) 3, 920, 2011.

12. F. He, X. Chen, and M. Pei Reconstruction of an in vitro tissue-specific microenvironment to rejuvenate synovium-derived stem cells for cartilage tissue engineering. Tissue Eng Part A 15, 3809, 2009.

13. M. Pei, F. He, and V.L. Kish Expansion on extracellular matrix deposited by human bone marrow stromal cells facilitates stem cell proliferation and tissue-specific lineage potential. Tissue Eng Part A 17, 3067, 2011.

14. C.D. Oh, S.H. Chang, Y.M. Yoon, S.J. Lee, Y.S. Lee, S.S. Kang, et al. Opposing role of mitogen-activated protein kinase subtypes, erk-1/2 and p38, in the regulation of chondrogenesis of mesenchymes. J Biol Chem 275, 5613, 2000.

15. Y. Zhang, T. Pizzute, and M. Pei A review of crosstalk between MAPK and Wnt signals and its impact on cartilage regeneration. Cell Tissue Res 358, 633, 2014.

16. J. Li, K.C. Hansen, Y. Zhang, C. Dong, C.Z. Dinu, M. Dzieciatkowska, et al. Rejuvenation of chondrogenic potential in a young stem cell microenvironment. Biomaterials 35, 642, 2014. 
17. Y. Zhang, T. Pizzute, J.T. Li, F. He, and M. Pei sb203580 preconditioning recharges matrixexpanded human adult stem cells for chondrogenesis in an inflammatory environment-a feasible approach for autologous stem cell based osteoarthritic cartilage repair. Biomaterials 64, $88,2015$.

18. Y. Zhang, J.T. Li, M.E. Davis, and M. Pei Delineation of in vitro chondrogenesis of human synovial stem cells following preconditioning using decellularized matrix. Acta Biomater 20, 39, 2015.

19. K. Itahana, J. Campisi, and G.P. Dimri Mechanisms of cellular senescence in human and mouse cells. Biogerontology 5, 1, 2004.

20. P. Atadja, H. Wong, I. Garkavtsev, C. Veillette, and K. Riabowol Increased activity of p53 in senescing fibroblasts. Proc Natl Acad Sci U S A 92, 8348, 1995.

21. K. Itahana, G. Dimri, and J. Campisi Regulation of cellular senescence by p53. Eur J Biochem 268, 2784, 2001.

22. A. Noda, Y. Ning, S.F. Venable, O.M. Pereira-Smith, and J.R. Smith Cloning of senescent cell-derived inhibitors of DNA synthesis using an expression screen. Exp Cell Res 211, 90, 1994.

23. J.P. Brown, W. Wei, and J.M. Sedivy Bypass of senescence after disruption of p21CIP1/WAF1 gene in normal diploid human fibroblasts. Science 277, 831, 1997.

24. G. Bianchi, A. Banfi, M. Mastrogiacomo, R. Notaro, L. Luzzatto, R. Cancedda, et al. Ex vivo enrichment of mesenchymal cell progenitors by fibroblast growth factor 2. Exp Cell Res 287, 98, 2003. 
25. S. Yanada, M. Ochi, K. Kojima, P. Sharman, Y. Yasunaga, and E. Hiyama Possibility of selection of chondrogenic progenitor cells by telomere length in FGF-2-expanded mesenchymal stromal cells. Cell Prolif 39, 575, 2006.

26. H. Zhao, S. Li, D. Han, V. Kaartinen, and Y. Chai Alk5-mediated transforming growth factor $\beta$ signaling acts upstream of fibroblast growth factor 10 to regulate the proliferation and maintenance of dental epithelial stem cells. Mol Cell Biol 31, 2079, 2011.

27. S. Hagmann, B. Moradi, S. Frank, T. Dreher, P.W. Kämmerer, W. Richter, et al. FGF-2 addition during expansion of human bone marrow-derived stromal cells alters MSC surface marker distribution and chondrogenic differentiation potential. Cell Prolif 46, 396, 2013.

28. T. Ito, R. Sawada, Y. Fujiwara, Y. Seyama, and T. Tsuchiya FGF-2 suppresses cellular senescence of human mesenchymal stem cells by down-regulation of TGF-beta2. Biochem Biophys Res Commun 359, 108, 2007.

29. B. Sacchetti, A. Funari, S. Michienzi, S. Di Cesare, S. Piersanti, I. Saggio, et al. Selfrenewing osteoprogenitors in bone marrow sinusoids can organize a hematopoietic microenvironment. Cell 131, 324, 2007. Erratum in: Cell 133, 928, 2008.

30. T. Ito, R. Sawada, Y. Fujiwara, and T. Tsuchiya FGF-2 increases osteogenic and chondrogenic differentiation potentials of human mesenchymal stem cells by inactivation of TGF-beta signaling. Cytotechnology 56, 1, 2008.

31. M. Pei, J.T. Li, M. Shoukry, and Y. Zhang A review of decellularized stem cell matrix: a novel cell expansion system for cartilage tissue engineering. Eur Cell Mater 22, 333, 2011. 
32. D.D. Campbell, and M. Pei Surface markers for chondrogenic determination: a highlight of synovium-derived stem cells. Cells 1, 1107, 2012.

33. S. Lim, H. Cho, E. Lee, Y. Won, C. Kim, W. Ahn, et al. Osteogenic stimulation of human adipose-derived stem cells by pre-treatment with fibroblast growth factor 2. Cell Tissue Res 364, 137, 2016.

34. N. Quarto, and M.T. Longaker FGF-2 inhibits osteogenesis in mouse adipose tissue-derived stromal cells and sustains their proliferative and osteogenic potential state. Tissue Eng 12, 1405, 2006.

35. N. Quarto, D.C. Wan, and M.T. Longaker Molecular mechanisms of FGF-2 inhibitory activity in the osteogenic context of mouse adipose-derived stem cells (mASCs). Bone 42, 1040, 2008.

36. J. Jiao, Y. Dang, Y. Yang, R. Gao, Y. Zhang, Z. Kou, et al. Promoting reprogramming by FGF2 reveals that the extracellular matrix is a barrier for reprogramming fibroblasts to pluripotency. Stem Cells 31, 729, 2013.

37. S. Hosseini-Farahabadi, P. Geetha-Loganathan, K. Fu, S. Nimmagadda, H.J. Yang, and J.M. Richman Dual functions for WNT5A during cartilage development and in disease. Matrix Biol 32, 252, 2013.

38. V. Church, T. Nohno, C. Linker, C. Marcelle, and P. Francis-West Wnt regulation of chondrocyte differentiation. J Cell Sci 115, 4809, 2002. 
39. J.H. Ryu, and J.S. Chun Opposing roles of WNT-5A and WNT-11 in interleukin-1beta regulation of type II collagen expression in articular chondrocytes. J Biol Chem 281, 22039, 2006.

40. I. Sekiya, J.T. Vuoristo, B.L. Larson, and D.J. Prockop In vitro cartilage formation by human adult stem cells from bone marrow stroma defines the sequence of cellular and molecular events during chondrogenesis. Proc Natl Acad Sci U S A 99, 4397, 2002.

41. F. Qu, J. Wang, N. Xu, C. Liu, S. Li, N. Wang, et al. WNT3A modulates chondrogenesis via canonical and non-canonical Wnt pathways in MSCs. Front Biosci (Landmark Ed) 18, 493, 2013.

42. Y. Kawakami, N. Wada, S.I. Nishimatsu, T. Ishikawa, S. Noji, and T. Nohno Involvement of Wnt-5a in chondrogenic pattern formation in the chick limb bud. Dev Growth Differ 41, 29, 1999.

43. C. Surmann-Schmitt, N. Widmann, U. Dietz, B. Saeger, N. Eitzinger, Y. Nakamura, et al. Wif-1 is expressed at cartilage-mesenchyme interfaces and impedes Wnt3a-mediated inhibition of chondrogenesis. J Cell Sci 122, 3627, 2009.

44. B.H. Hoang Wnt, osteosarcoma, and future therapy. J Am Acad Orthop Surg 20, 58, 2012.

45. X. Qian, C. Zhang, G. Chen, Z. Tang, Q. Liu, J. Chen, et al. Effects of BMP-2 and FGF-2 on the osteogenesis of bone marrow-derived mesenchymal stem cells in hindlimb-unloaded rats. Cell Biochem Biophys 70, 1127, 2014. 
46. N. Ye, and D. Jiang Ghrelin accelerates the growth and osteogenic differentiation of rabbit mesenchymal stem cells through the ERK1/2 pathway. BMC Biotechnol 15, 51, 2015.

47. P. Zhang, Q. Dai, N. Ouyang, X. Yang, J. Wang, S. Zhou, et al. Mechanical strain promotes osteogenesis of BMSCs from ovariectomized rats via the ERK1/2 but not p38 or JNK-MAPK signaling pathways. Curr Mol Med 15, 780, 2015.

48. B.S. Kim, H.J. Kang, J.Y. Park, and J. Lee Fucoidan promotes osteoblast differentiation via JNK- and ERK-dependent BMP2-Smad 1/5/8 signaling in human mesenchymal stem cells. Exp Mol Med 47, e128, 2015. 
Figure 1:

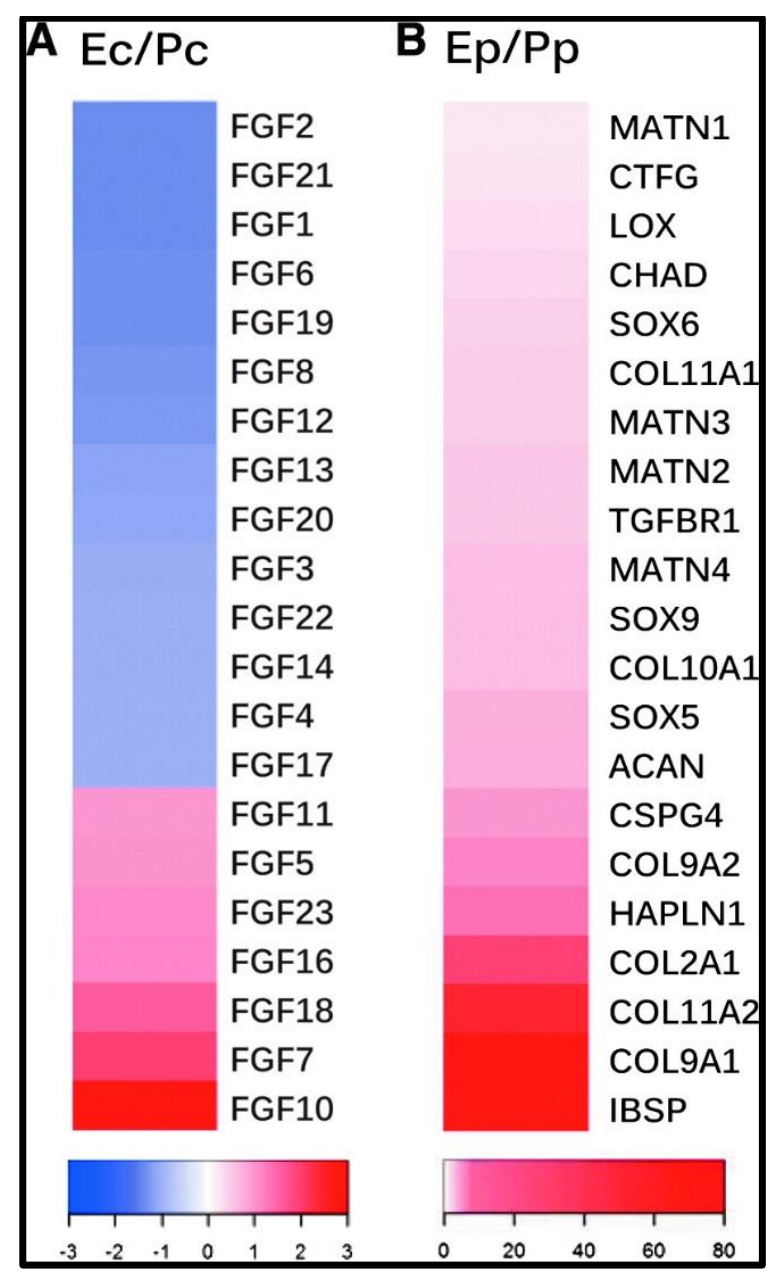


Figure 1: Gene expression of FGF ligands in cell expansion phase and chondrogenic markers in chondrogenic induction phase following the dECM pretreatment. The study design was detailed previously.18 Briefly, human SDSCs were expanded on either dECM or Plastic for one passage followed by a 2-week chondrogenic induction. Microarray analysis was used to evaluate FGF ligand genes in expanded cells (A) and chondrogenic marker genes in differentiated cells (B). The raw data were uploaded into Partek (St. Louis, MO) software for initial analysis. After raw intensity was background-subtracted, robust multiarray analysis was normalized, log transformed, and fold changes were determined. Heatmap. 2 in R was used to show the effects on FGF ligand and chondrogenic genes, annotated in Ingenuity Pathway Analysis (IPA, Redwood City, CA) as affecting cell proliferation and chondrogenic differentiation, respectively. dECM expanded cells were referred to as Ec, while Plastic expanded cells were referred to as Pc (A). The pellets from dECM expanded cells were referred to as Ep, while those from Plastic expanded cells were referred to as Pp (B). FGF, fibroblast growth factor; dECM, decellularized extracellular matrix; SDSC, synovium-derived stem cell. 
Figure 2:

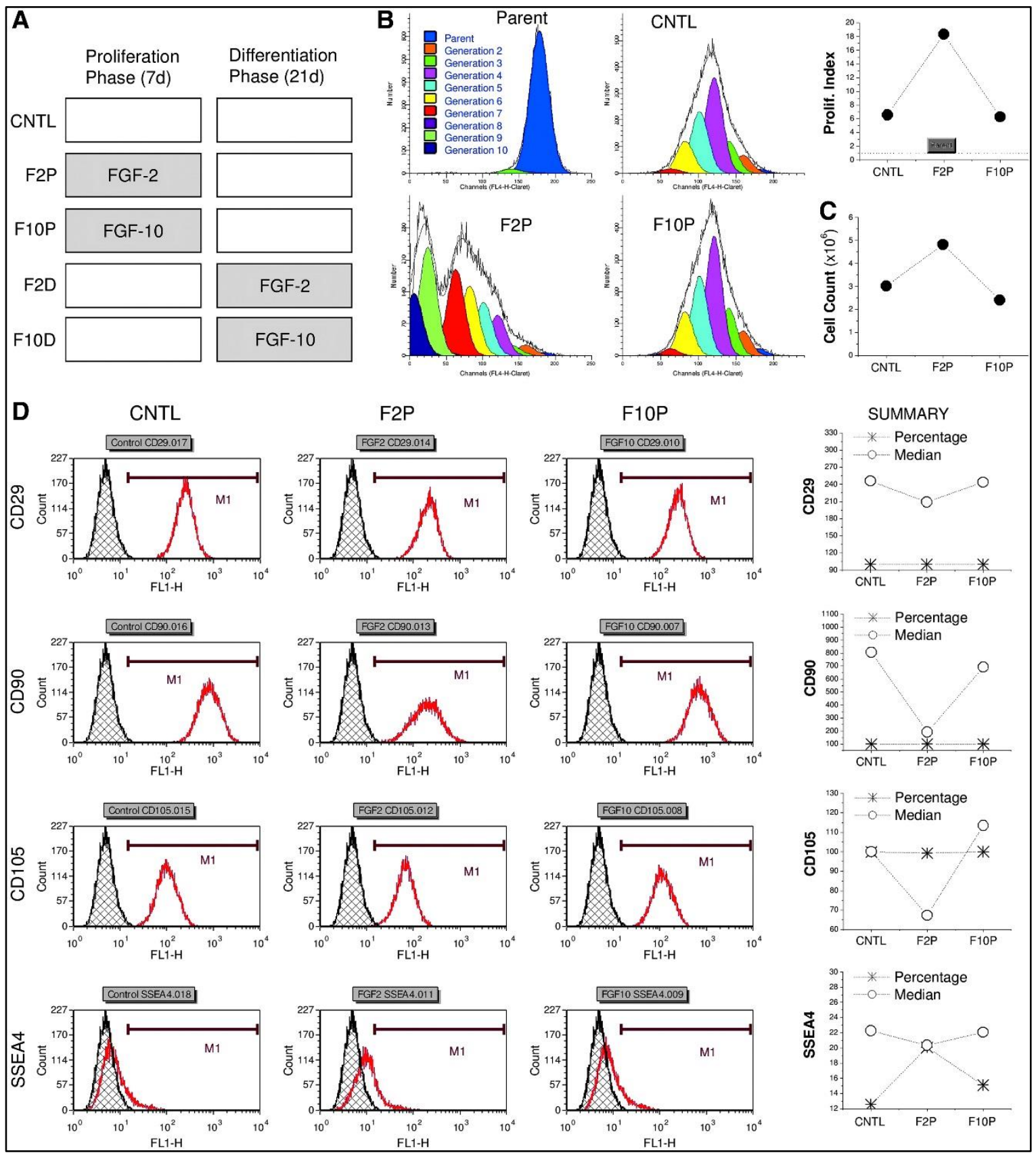


Figure 2: FGF ligand mediated human SDSC proliferation. (A) Experimental design. Five groups in total: FGF-2 and FGF-10 were only included in a 7-day cell expansion phase (F2P and F10P, respectively) or in a 21-day differentiation phase (F2D and F10D, respectively) with nonFGF treatment as a control (CNTL). (B) Flow cytometry was used to measure proliferation (prolif.) index of expanded SDSCs. (C) Cell number was counted $(\times 106)$ after a 7-day cell expansion on $175 \mathrm{~cm} 2$ flasks $(\mathrm{n}=6)$. (D). Flow cytometry was used to measure both percentage and median fluorescence intensity of mesenchymal stem cell surface markers (CD29, CD90, CD105, and SSEA4) of expanded SDSCs. SSEA4, stage-specific embryonic antigen 4. 
Figure 3:
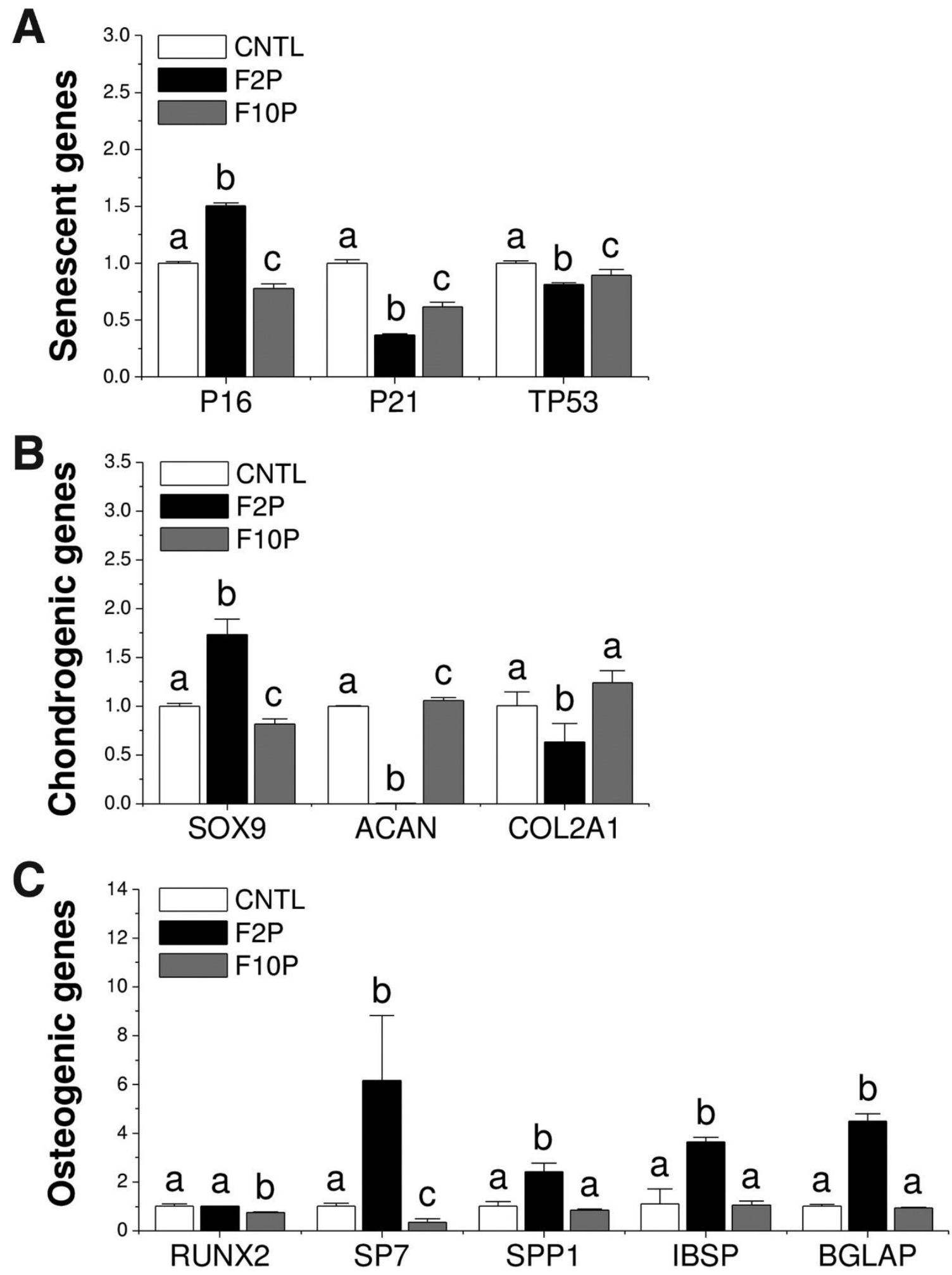
Figure 3: FGF ligand mediated senescence and differentiation-related gene expression in expanded SDSCs. TaqMan® real-time PCR was used to quantify mRNA levels of senescent genes (A): P16, P21, and TP53, chondrogenic genes (B): SOX9, ACAN, and COL2A1, and osteogenic genes (C): RUNX2, SP7, SPP1, IBSP, and BGLAP. Data are shown as average \pm SD for $n=4$. Groups not connected by the same letter are significantly different $(p<0.05)$. PCR, polymerase chain reaction; SD, standard deviation; SOX9, SRY (sex determining region Y)-box 9; ACAN, aggrecan; COL2A1, type II collagen; RUNX2, Runt-related transcription factor 2; SP7, Osterix; SPP1, secreted phosphoprotein 1; IBSP, bone sialoprotein; BGLAP, bone gammacarboxyglutamate (Gla) protein. 
Figure 4:

A

Day 10

\begin{tabular}{lll}
\hline Pellet & GAG
\end{tabular}

CNTL

F2P

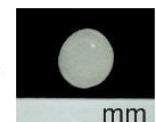

F2D

179
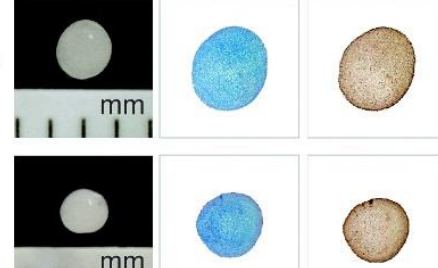

F10P

I I I I
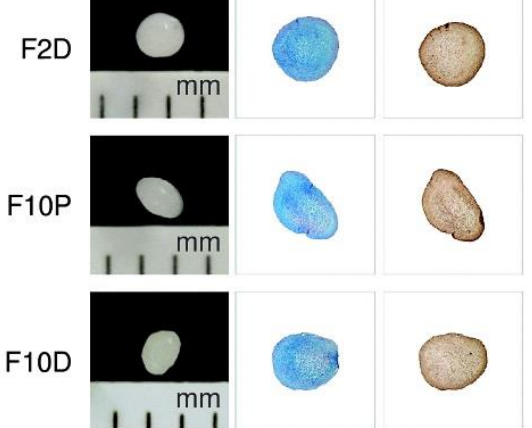

Day 21

Pellet GAG Col2

(mm)

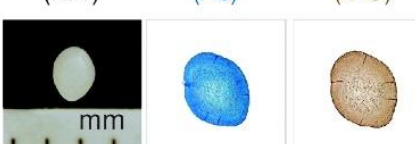

l 1 1 1
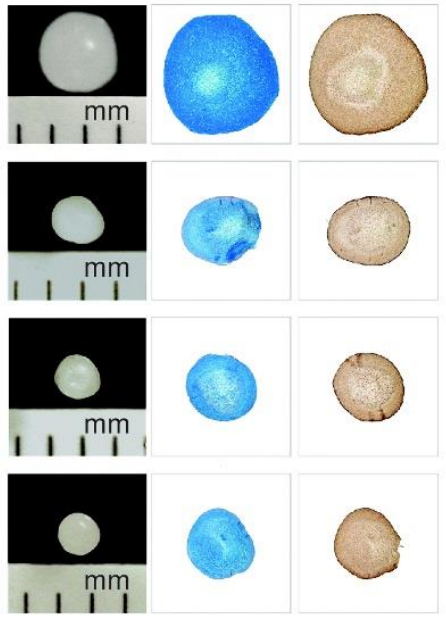
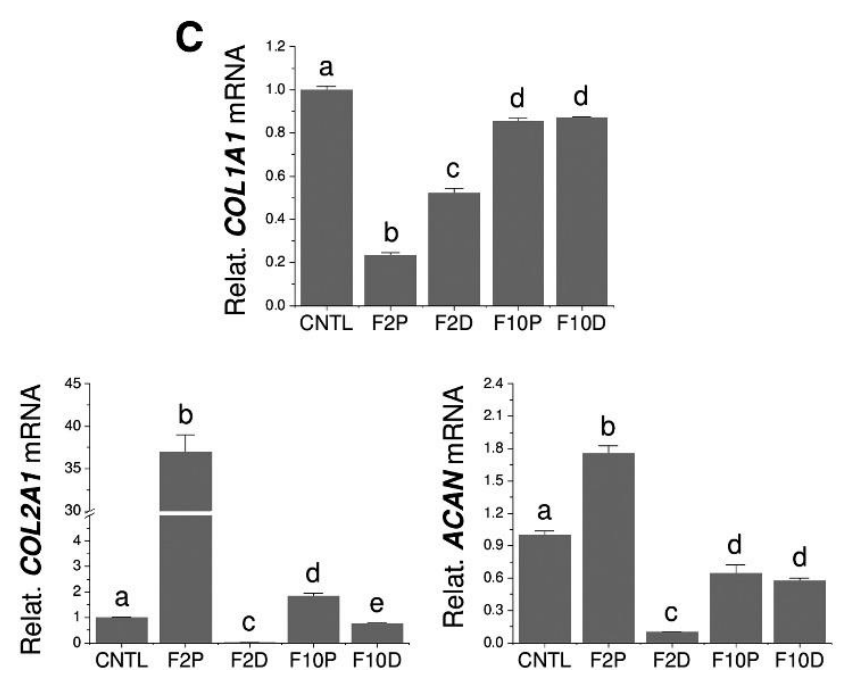

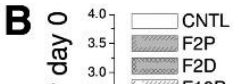

ठे $2.5-\mathrm{F} 10 \mathrm{P}$

ลे $2.5-$ F10
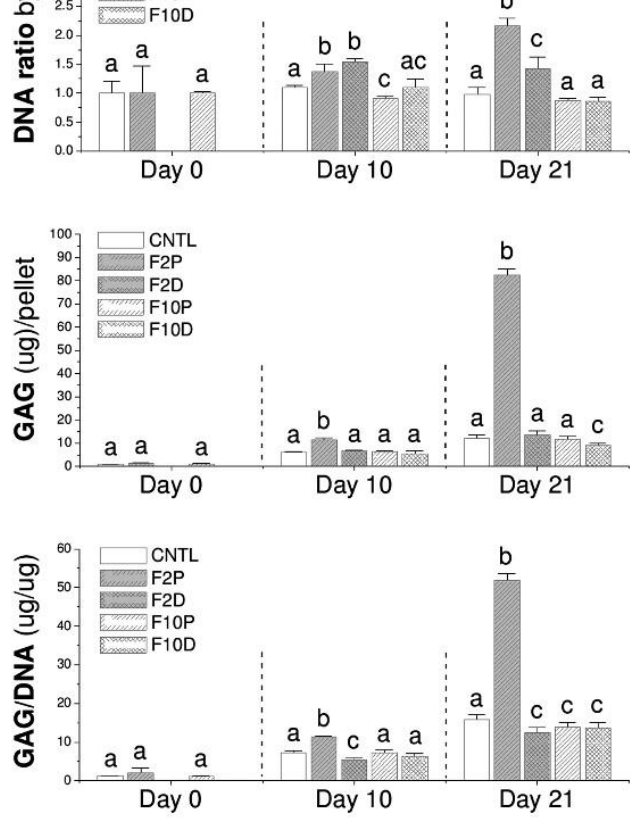

D
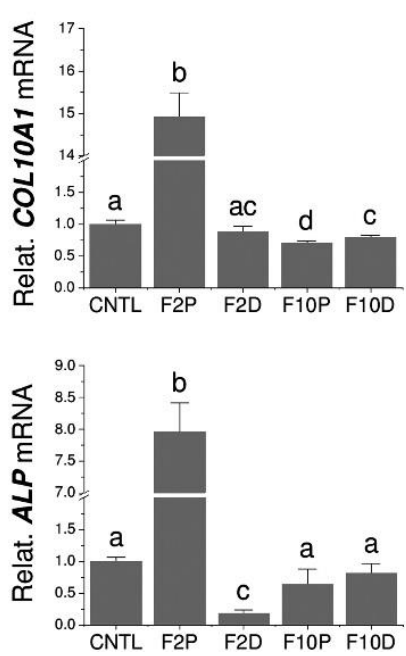
Figure 4: FGF ligand mediated SDSC chondrogenic differentiation. After a 21-day chondrogenic induction, SDSC pellets were evaluated using Alcian blue staining for sulfated GAGs and immunohistochemical staining for Col2 (A), biochemical analysis for both DNA and GAG amounts in a pellet (B), and TaqMan real-time PCR for mRNA levels of chondrogenic genes (C): COL1A1, COL2A1, and ACAN and hypertrophic genes (D): COL10A1 and ALP. Data are shown as average $\pm \mathrm{SD}$ for $\mathrm{n}=4$. Groups not connected by the same letter are significantly different $(\mathrm{p}<0.05)$. GAG, glycosaminoglycan; Col2, type II collagen; ALP, alkaline phosphatase. 
Figure 5:
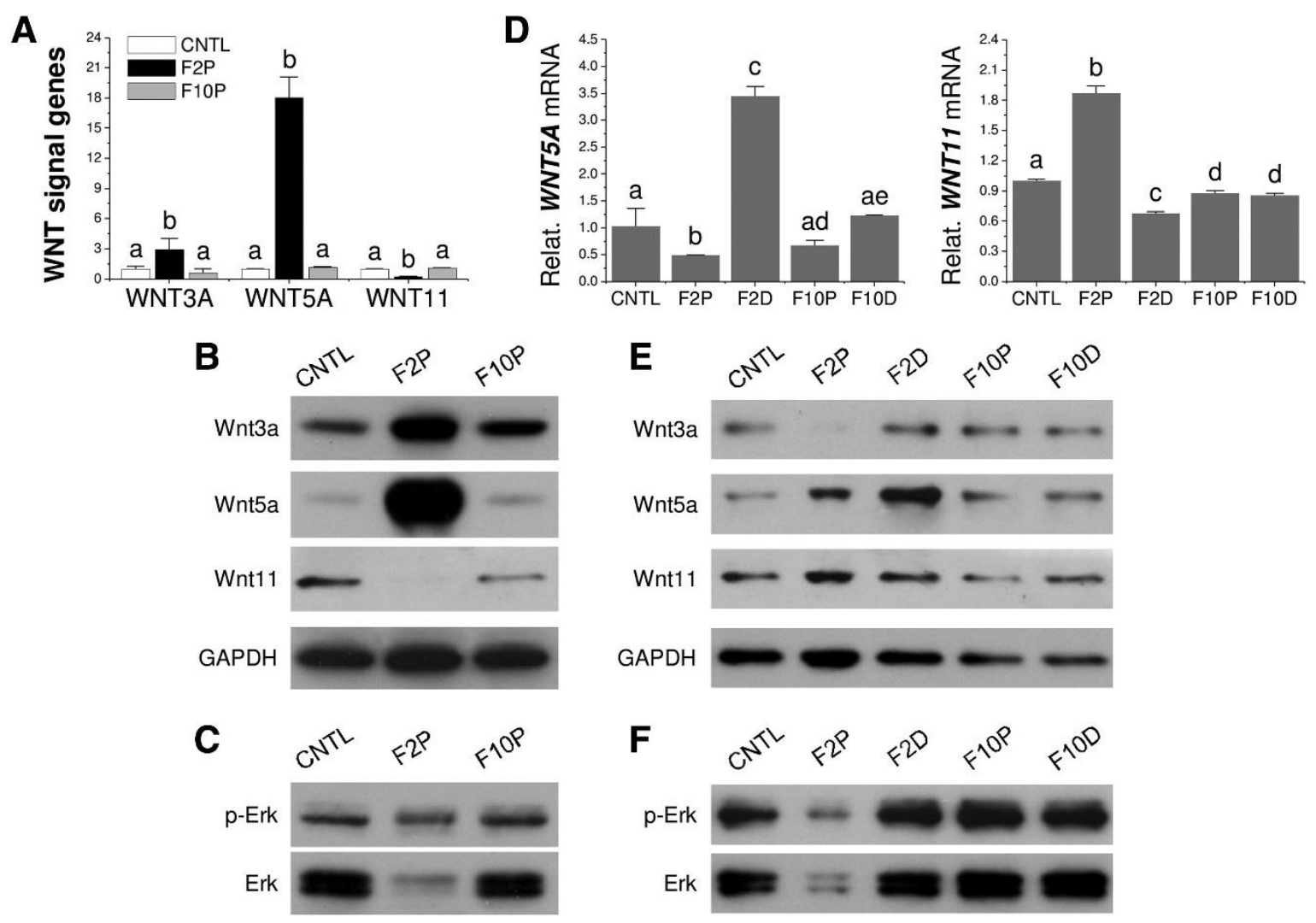

F $\quad a^{24}<2^{2}<20<10^{\circ}<10^{00}$
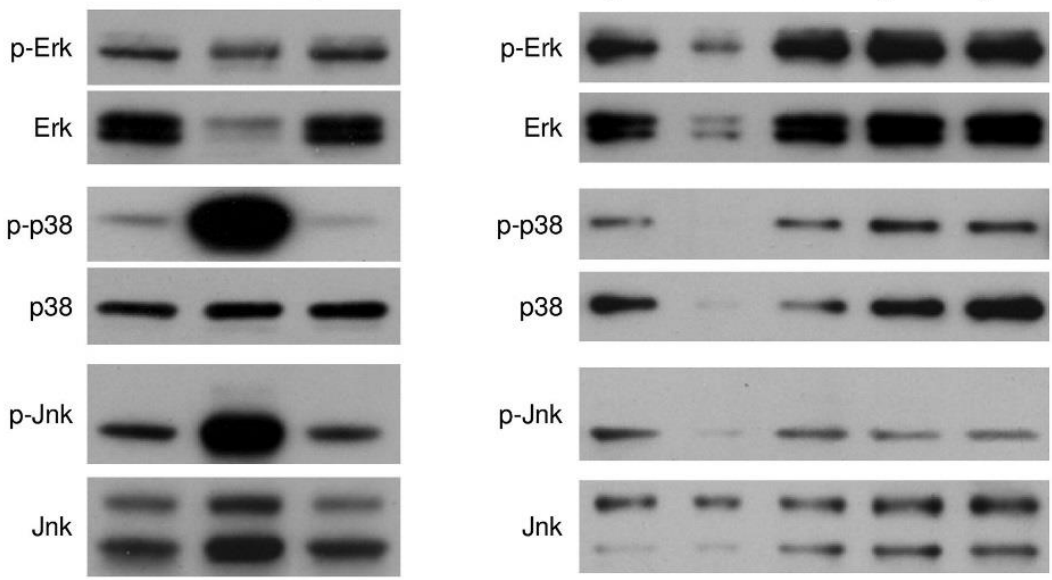

GAPDH

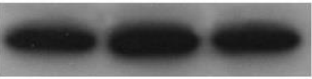

GAPDH 
Figure 5: FGF ligand mediated Wnt and MAPK signal changes in SDSC chondrogenesis. During cell expansion (A-C) and chondrogenic induction (D-F), Wnt signals were evaluated using both TaqMan real-time PCR (A, D) and Western blot (B, E), while MAPK signals were evaluated using Western blot (C, F). GAPDH was used as an internal control. Data are shown as average $\pm \mathrm{SD}$ for $\mathrm{n}=4$. Groups not connected by the same letter are significantly different $(\mathrm{p}<0.05)$. MAPK, mitogen-activated protein kinase; GAPDH, glyceraldehyde-3-phosphate dehydrogenase. 
Figure 6:

A

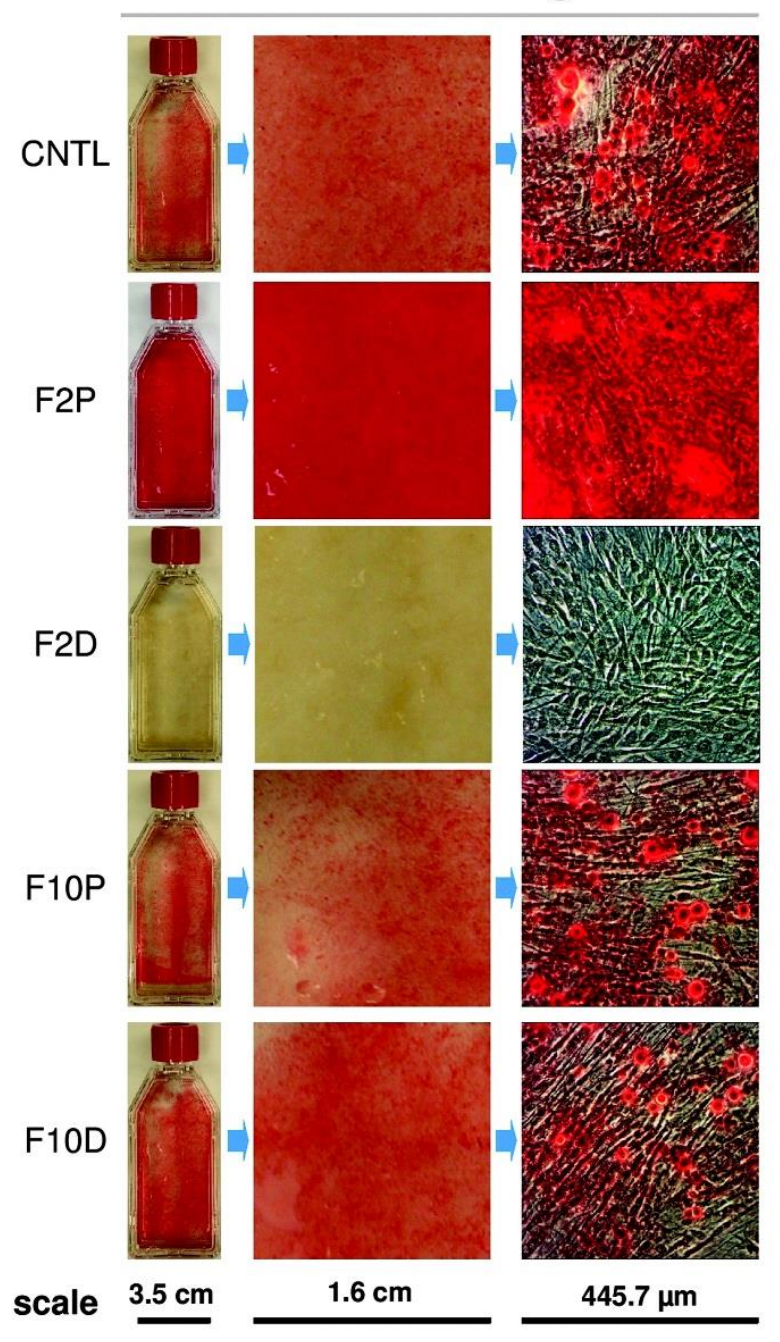

B

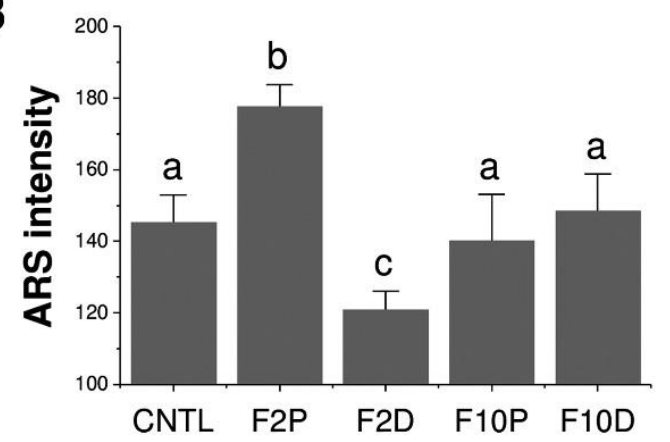

C ALP staining

CNTL
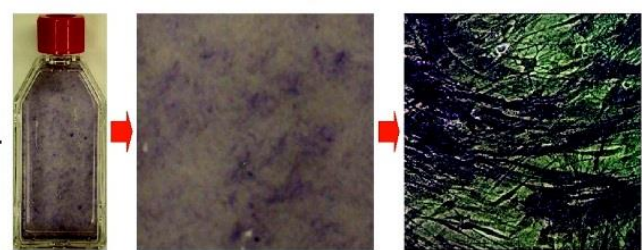

F2P
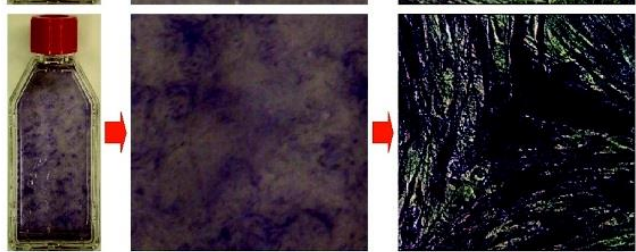

F2D
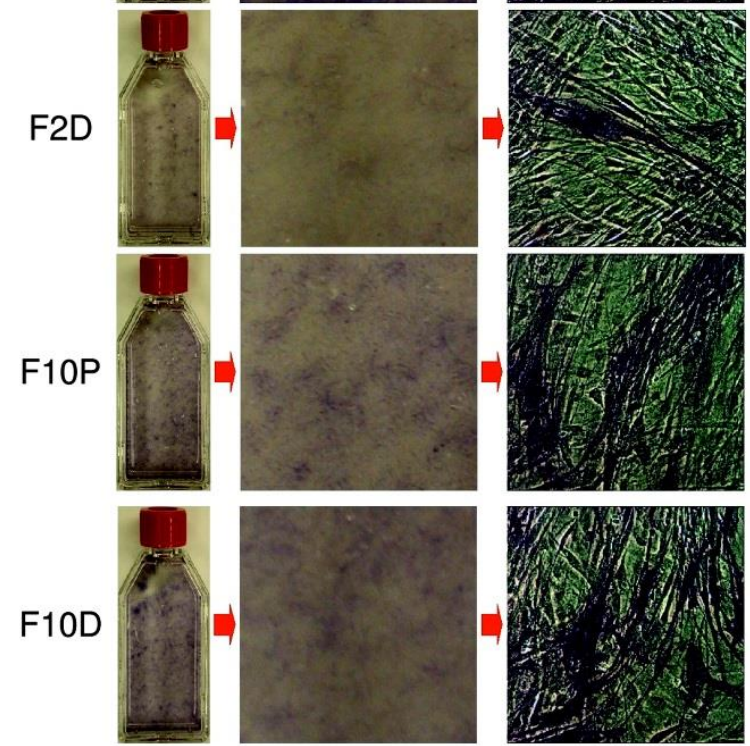

scale $3.5 \mathrm{~cm}$

$1.6 \mathrm{~cm}$

$445.7 \mu \mathrm{m}$

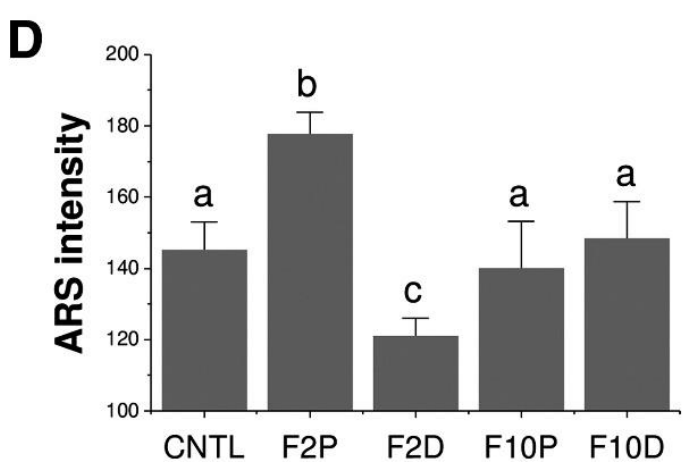


Figure 6: FGF ligand mediated SDSC osteogenic differentiation. After a 21-day osteogenic induction, both ARS (A) and ALP staining (C) were used to evaluate osteogenic differentiation. ImageJ software was used to semiquantify the density of staining (B, D, respectively). Data are shown as average \pm SD for $n=4$. Groups not connected by the same letter are significantly different $(\mathrm{p}<0.05)$. ARS, Alizarin Red S. 


\section{Chapter 3:}

\section{Noncanonical WNT signals promote the early}

\section{redifferentiation of human nucleus pulposus cells}

In preparation for submission; TBD 2017

Tyler Pizzute ${ }^{1,2}$, Xiao-Bing Zhang ${ }^{3 *}$, Ming Pei ${ }^{1,2,4^{* *}}$

${ }^{1}$ Stem Cell and Tissue Engineering Laboratory, Department of Orthopaedics, West Virginia University, Morgantown, WV, USA; ${ }^{2}$ Exercise Physiology, West Virginia University, Morgantown, WV, USA; ${ }^{3}$ Department of Medicine, Loma Linda University, Loma Linda, CA, USA; ${ }^{4}$ Mary Babb Randolph Cancer Center, Robert C. Byrd Health Sciences Center, West Virginia University, Morgantown, WV, USA

Running Head: WNT Signals and Disc Regeneration

*Co-Corresponding Author: Xiao-Bing Zhang, PhD, Division of Regenerative Medicine MC 1528B, Department of Medicine, Loma Linda University, 11234 Anderson Street, Loma Linda, CA 92350, USA, Email: xzhang@1lu.edu

**Corresponding author: Ming Pei MD, PhD, Stem Cell and Tissue Engineering Laboratory, Department of Orthopaedics, West Virginia University, PO Box 9196, One Medical Center Drive, Morgantown, WV 26506-9196, USA, Telephone: 304-293-1072; Fax: 304-293-7070; Email: mpei@hsc.wvu.edu 


\begin{abstract}
Cell expansion and preconditioning strategies are a growing area of interest for therapies targeting intervertebral discs (IVDs), primarily aimed at increasing rejuvenation and proliferative capacities of nucleus pulposus cells (NPCs). Although therapies designed for such applications seem promising, it is unknown whether the signals and potential therapeutic targets of the Wnt pathways previously implicated in chondrogenesis, Wnt3A, Wnt5A, and Wnt11, play further key roles in the promotion or regulation of human NPC redifferentiation. In this study, NPCs were harvested and isolated from herniated disc patients, cultured in vitro, and transduced with lentiviral vectors that overexpress the WNT3A, WNT5A, and WNT11 genes, or Cas9/sgRNA vectors that knock out these genes. Following expansion, transduced NPCs, for both gene knockout and overexpression groups, were redifferentiated toward the NP phenotype and assessed via flow cytometry, quantitative polymerase chain reaction (qPCR) for redifferentiation genes, and biochemically for glycosaminoglycan (GAG) content. The overexpression of specific WNT factors led to increases in both GAG deposition and expression of redifferentiation genes. These effects were attenuated by CRISPR-Cas9 vectors targeting these same WNT genes. These results indicate that specific WNT signals can regulate the expression of redifferentiation genes, unequally impact GAG deposition, and contribute to the redifferentiation of human NPCs.
\end{abstract}

Keywords: nucleus pulposus, wnt signaling, intervertebral disc, lentivirus, CRISPR-Cas9 


\section{Introduction}

Located inside the intervertebral disc (IVD), nucleus pulposus (NP) tissue provides structural support and shock absorbing capacity along the spinal column (Urban and Roberts, 1995). NP tissue functions through a hydrated collagen and proteoglycan-rich extracellular matrix. Unfortunately, IVD herniation and degeneration are among the most prevalent causes for back pain; interestingly, low back and neck pain represented the third-highest amount of US health care spending from 1996 to 2013 (Dieleman et al., 2016). These conditions can negatively impact the properties and function of the IVD due to the loss of disc height, NP cellularity, matrix remodeling, cell dedifferentiation, and other deleterious biochemical conditions (Bowles and Setton, 2017; Hegewald et al., 2011; Hiyama et al., 2010; McCann and Seguin, 2016; Tworney and Taylor, 1987).

Recently, the preconditioning of NP cells (NPCs) and autologous NPC-mediated therapies have aimed to rejuvenate and replenish the depleted NP tissue following injury or degeneration (Hegewald et al., 2011; Pei, 2017; Shoukry et al., 2013). However, many of the biochemical pathways and signals that allow for successful redifferentiation and therapeutic targeting have yet to be fully elucidated (Srivastava et al., 2017). These are well-conserved pathways which have been extensively studied and implicated in tissue homeostasis (Arai et al., 2012), NP apoptosis (Wang et al., 2016), and, more specifically, in cell migration and differentiation (Garcin \& Habib, 2017), with particularly notable roles in chondrogenesis (Green et al., 2015; Pizzute et al., 2016; Zhong et al., 2015). The roles of both canonical and noncanonical Wnt (Wingless protein family) signals in chondrocytic tissues have been extensively reviewed (Mohammad et al., 2016; Zhang et al., 2014) and are well-demonstrated regulators in the development and differentiation of these cells into mature tissues. 
From a degenerative standpoint, Wnt/ $\beta$-catenin pathway signals have been implicated in canine IVD degeneration (Smolders et al., 2013), as well as murine IVD aging, degeneration, and development (Dahia et al., 2009; Jia et al., 2016; Kondo et al., 2011). More recently, the abnormalities caused by Wnt signaling have also been associated with local inflammation and IVD ossification (Shao et al., 2017). In a study by Winkler and colleagues, canonical Wnt signals were shown to be decreased, along with Sonic hedgehog (Shh) signaling in the aging human adult disc, and it was suggested that the Wnt pathway could be a therapeutic target for disc degeneration (Winkler et al., 2014). Although several studies have suggested that Wnt signals play key roles in human disc, there are fewer studies using human-derived IVDs or NPCs to fully support such hypotheses, especially studies that directly compare multiple Wnt signals at once during NPC redifferentiation.

Furthermore, recently published studies have suggested that the progression of degenerative disc disease may be related to the crosstalk between Wnt and TGF- $\beta$ signaling (Cleary et al., 2015; Matta et al., 2017), This signaling, a well-known differentiation stimulating signal, occurs naturally in cartilaginous tissues and is commonly used to perpetuate chondrogenic and redifferentiated NPC phenotypes in vitro (Cai et al., 2017; Chen et al., 2006). Additionally, Wnt3A, Wnt5A, and Wnt11 have each been shown to be involved in mesenchymal stem cell (MSC) differentiation. Wnt3A has been implicated in MSC proliferation, increasing differentiation potential, and suppression of differentiation (Buchtova et al., 2015; Lee et al., 2016; Narcisi et al., 2015; Narcisi et al., 2016), while Wnt5A and Wnt11 have been more extensively implicated in differentiation and cartilage development (Hosseini-Farahabadi et al., 2012; Liu et al., 2014; Pizzute et al., 2016; Thorfve et al., 2012; Usami et al., 2016; Zhu et al., 2012). 
Due to the identified roles that Wnt signaling pathways play in cartilage and stem cell differentiation (Cleary et al., 2015; Hosseini-Farahabadi et al., 2012; Pizzute et al., 2016; Zhang et al., 2014), as well as the inherent similarities and shared properties between the chondrogenic differentiation of chondrocytic tissues (Chen et al., 2017), such as those derived from the synovium (synovium-derived stem cells or SDSCs) with NPC redifferentiation (Shoukry et al., 2013), we hypothesize that specific Wnt signals are also significant regulators of NP redifferentiation in humans. We expect that noncanonical signals, Wnt5A and Wnt11, will each exhibit a more dramatic effect on NPC redifferentiation than canonical Wnt3A. 


\section{Materials and Methods}

\section{Cloning of overexpression and knockout vectors}

We used VSV-G pseudotyped lentiviral vectors in this study. To construct overexpression (OE) vectors, the open reading frames (ORF) sequences of human WNT3A, WNT5A, and WNT11 genes or control green fluorescence protein (GFP) were cloned into a lentiviral vector backbone Lenti SFFV-X-Wpre by Gibson Assembly® from New England BioLabs Inc. (NEB, Ipswich, MA). WNT3A, WNT5A, and WNT11 cDNAs were purchased from DNASU Plasmid Repository (Tempe, AZ). To knock out human WNT3A, WNT5A, and WNT11, we cloned the spacer sequence of single-guide RNA (sgRNA) into backbone Lenti-U6-sgRNA-SFFV-Cas9-E2A-Puro, in which the U6 promoter drives the expression of sgRNA and the spleen focus-forming virus (SFFV) promoter drives the expression of both CRISPR associated protein 9 (Cas9) and puromycin-resistant gene. The spacer sequences are as follows: sgWNT3A-1, GGCCATCGCCGCGCGAG; $\quad$ sgWNT3A-2， GCAGCTACCCGATCTGG； sgWNT3A-2， GGCACGGCCGCCATCTG; sgWNT5A-1， GACGGCCTTCACATACG; $\quad$ sgWNT5A-2, GGATGCGCTCCCGCTCG; $\quad$ sgWNT5A-3， GCTCACCGCGTATGTGA; $\quad$ sgWNT11A-1， GGGGGCGAGCTCAATGG; sgWNT11A-2， GGCGTGGCTGATGGCGG; sgWNT11A-3, GTGGCTCACCTGGGACG. The sgRNA design and vector cloning have been detailed elsewhere (Zhang et al., 2016; Zhang et al., 2017a). All the inserts were confirmed by Sanger sequencing. The DNA-calcium phosphate co-precipitation method was used for packaging lentiviral vectors. After 100-fold concentration by ultracentrifugation, the biological titers of vectors were determined by transducing HT1080 cells.

\section{Cell culture and lentiviral transduction}


Human adult NP cells were obtained from the NP tissue of herniated disc patients following patient consent and Institutional Review Board (IRB) approval. Cells were cultured in T25 cell culture flasks and growth medium containing Alpha Minimum Essential Medium ( $\alpha$ MEM), 10\% fetal bovine serum (FBS), $100 \mathrm{U} / \mathrm{mL}$ penicillin, $100 \mu \mathrm{g} / \mathrm{mL}$ streptomycin, and $0.25 \mu \mathrm{g} / \mathrm{mL}$ fungizone.

Lentiviral vectors were produced by previously described methods (Meng et al., 2012; Zhang et al., 2016). After reaching 40-60\% confluence in cell culture flasks, cell numbers were visually estimated based upon apparent density and lentiviral vectors targeting each respective WNT-gene were added at a multiplicity of infection (MOI) of 2 in $\alpha$ MEM containing $10 \%$ FBS, and $1 \times$ Protamine Sulfate (PS) and incubated at $37^{\circ} \mathrm{C}$ under $21 \%$ oxygen in a cell culture incubator for 24 h. After $24 \mathrm{~h}$, viral vector-containing medium was removed and normal growth medium was added for cell expansion. For CRISPR-Cas9 vectors, an additional selection for 4 days was performed with puromycin-enriched $(1 \mathrm{ug} / \mathrm{mL})$ selection culture media to kill non-transduced cells and increase efficiency. GFP expression, visualized by an Invitrogen ${ }^{\mathrm{TM}}$ EVOS $^{\mathrm{TM}}$ FL Auto Imaging System (Thermo Fisher Scientific, Waltham, MA) at 24, 48, and $72 \mathrm{~h}$, was used as a positive control for viral transduction.

\section{Cell proliferation}

To determine the proliferation index of each group, before cell expansion, NPCs were labeled with CellVue ${ }^{\circledR}$ Claret at $2 \times 10-6 \mathrm{M}$ for $5 \mathrm{~min}$ according to the manufacturer's protocol (Sigma-Aldrich, St. Louis, MO). After six days of proliferation, expanded cells were collected and measured using a BD FACS LSRFORTESSA $\square$ flow cytometer (BD Biosciences, San Jose, CA). Twenty thousand events of each sample were collected using CellQuest Pro software (BD Biosciences); cell 
proliferation index was analyzed using ModFit LT $\square$ version 3.1 (Verity Software House, Topsham, ME).

\section{Redifferentiation induction and evaluation}

Expanded cells $(3 \times 105)$ were centrifuged at $500 \mathrm{~g}$ for $5 \mathrm{~min}$ in a $15-\mathrm{mL}$ polypropylene tube to form a pellet. After overnight incubation (day 0), the pellets were cultured in a serum-free chondrogenic medium consisting of high-glucose Dulbecco's Modified Eagle's Medium (DMEM), $40 \mu \mathrm{g} / \mathrm{mL}$ proline, $100 \mathrm{nM}$ dexamethasone, $100 \mathrm{U} / \mathrm{mL}$ penicillin, $100 \mu \mathrm{g} / \mathrm{mL}$ streptomycin, $0.1 \mathrm{mM}$ L-ascorbic acid-2-phosphate, and 1×ITS ${ }^{\mathrm{TM}}$ Premix (BD Biosciences) with the supplementation of $10 \mathrm{ng} / \mathrm{mL}$ transforming growth factor beta3 (TGFß3) (PeproTech, Inc., Rocky Hill, NJ) in a 5\% O2 incubator as long as 28 days. The pellets were evaluated for NPC redifferentiation using biochemical analysis and quantitative real-time polymerase chain reaction (qPCR).

Representative pellets ( $\mathrm{n}=4$ ) were digested at $60^{\circ} \mathrm{C}$ for $4 \mathrm{~h}$ with $125 \mu \mathrm{g} / \mathrm{mL}$ papain in PBE buffer (100 mM phosphate, $10 \mathrm{mM}$ ethylenediaminetetraacetic acid, $\mathrm{pH}$ 6.5) containing $10 \mathrm{mM}$ cysteine. To quantify cell density, the amount of DNA in the papain digestion was measured using the QuantiT $\square$ PicoGreen ${ }^{\circledR}$ dsDNA assay kit (Life Technologies, Carlsbad, CA) with a CytoFluor ${ }^{\circledR}$ Series 4000 (Applied Biosystems, Foster City, CA). GAG was measured using dimethylmethylene blue (DMMB) dye and a Spectronic $\square$ BioMate $\square 3$ Spectrophotometer (Thermo Fisher Scientific) with bovine chondroitin sulfate (Sigma-Aldrich) as a standard.

Total RNA was extracted from representative samples $(n=4)$ using an RNase-free pestle in TRIzol® (Life Technologies). Two micrograms of mRNA were used for reverse transcriptase with High-Capacity cDNA Archive Kit (Applied Biosystems) at $37^{\circ} \mathrm{C}$ for $120 \mathrm{~min}$. NPC 
redifferentiation related genes, type I collagen (COL1A1; Assay ID Hs00164004_m1), aggrecan (ACAN; Assay ID Hs00153936_m1), type II collagen (COL2A; Assay ID Hs00156568_m1), paired box 1 (PAX1; Assay ID Hs01071293_g1), and forkhead box F1 (FOXF1; Assay ID Hs00230962_m1) were customized by Applied Biosystems as part of their Custom TaqMan ${ }^{\circledR}$ Gene Expression Assays. Wnt signals were also evaluated using qPCR (WNT3A; Assay ID Hs00263977_m1, WNT5A; Assay ID Hs00998537_m1, and WNT11; Assay ID Hs00182986_m1) following cell expansion of virally transduced cells and after NP redifferentiation. Glyceraldehyde-3-Phosphate Dehydrogenase (GAPDH; Assay ID Hs02758991_g1) was carried out as the endogenous control gene. TaqMan ${ }^{\circledR}$ real-time PCR was performed with iCycler iQ $\square$ Multi Color Real-Time PCR Detection and calculated by computer software (Perkin-Elmer, Wellesley, MA). Relative transcript levels were calculated as $\chi=2-\Delta \Delta \mathrm{Ct}$, in which $\Delta \Delta \mathrm{Ct}=\Delta \mathrm{E}-\Delta \mathrm{C}$, $\Delta \mathrm{E}=\mathrm{Ctexp}-\mathrm{CtGAPDH}$, and $\Delta \mathrm{C}=\mathrm{Ctct} 1-\mathrm{CtGAPDH}$. 


\section{Statistical analysis}

Analysis of variance (ANOVA) analysis with pairwise comparison and t-test was used to compare measurements between different groups. All statistical analyses were performed with SPSS 13.0 statistical software (SPSS Inc., Chicago, IL). p values less than 0.05 were considered statistically significant. 


\section{Results}

Lentiviral vectors effectively overexpressed WNT genes

The lentiviral vectors were successfully transduced with protamine sulfate (PS) supplemented in culture media to increase transduction efficiency, which did not cause any observable differences in cell morphology or proliferation. The successful transduction of a GFP-containing vector in NPCs was demonstrated using immunofluorescence with a greater intensity of green color in the transduced cells at the MOI of five versus one (Fig. 1A). MOI of two was chosen for this study given the notable success in WNT gene expression in the MOI of one group and a potential reduction of cell death and proliferation capacity. Our qPCR result suggested that both PS supplementation and GFP transduction did not result in WNT gene expression and either WNT gene transduction did not cause a significant change of another WNT signal (Fig. 1B). A successful transduction of three WNT signals (WNT3A, WNT5A, and WNT11) was verified in both expanded cells (Fig. 1B) and the following redifferentiation induced pellets for up to 28 days (Fig. 1C). WNT3A-OE was the only group that expressed any detectable WNT3A mRNA at each timepoint (Fig. 1C). Similarly, WNT5A-OE expressed 500-2800-fold greater WNT5A mRNA compared to controls (CNTL) during redifferentiation (Fig. 1C), and WNT11-OE expressed upwards of 25,000-fold greater WNT11 mRNA (Fig. 1C) at each timepoint during redifferentiation induction in pellet culture versus CNTL.

Overexpression of WNT3A enhanced NPC proliferation

Proliferation Index (PI) and generational analysis of stained NPCs by flow cytometry (Fig. 2) revealed that WNT3A-OE $(\mathrm{PI}=10.65)$ caused the greatest increase in proliferation versus $\mathrm{CNTL}$ $(\mathrm{PI}=6.70) . \mathrm{WNT} 5 \mathrm{~A}-\mathrm{OE}(\mathrm{PI}=3.74)$ proliferated significantly less than $\mathrm{CNTL}$ and WNT11-OE 
$(\mathrm{PI}=7.48)$ was slightly elevated compared to CNTL. Also, CNTL, WNT5A-OE, and WNT11-OE predominantly proliferated to the third generation, wheareas WNT3A-OE produced a peak in the fourth generation.

Overexpression of noncanonical WNT signals promoted NPC redifferentiation

After viral transduction and cell expansion, all WNT-OE groups significantly decreased COL1A1 expression versus CNTL; however, in the condensation at day 0 of redifferentiation, all WNT transduced groups exhibited a significant increase of COL1A1 expression, which later attenuated for up to 28 days (Fig. 3A). For NPC markers, despite a lower expression in cell expansion phase, PAX1 expression significantly increased in day 0 pellets of all WNT-OE cells but not in the following chondrogenic induced pellets except the WNT11-OE group at day 11 (Fig. 3B). Other marker genes, such as FOXF1 (Fig. 3C) and COL2A1 (Fig. 3D), were detected a significant increase in both WNT5A-OE and WNT11-OE groups at day 11 but not at day 28. Interestingly, the WNT11-OE group also exhibited a significant increase of FOXF1 and COL2A1 in day 0 pellets (Fig. 3C/D), indicating that noncanonical WNT signals, particularly WNT11, play a critical role in the early stage of NPC redifferentiation. This finding was further corroborated by ACAN expression in NPCs, which significantly increased in all WNT-OE groups at all pellet culture time points except no significant change at day 28 in the WNT11-OE group (Fig. 3E).

Despite no visible difference in the size of all pellet groups (Fig. 4A), the overexpression of noncanonical signals, WNT5A-OE and WNT11-OE, exhibited significantly increased GAG/DNA ratios at day 28 versus CNTL, but not in canonical WNT3A-OE (Fig. 4B). Similarly, WNT5A-OE exhibited the greatest raw GAG content per pellet at day 28 (Fig. 4C) versus CNTL. Interestingly, 
WNT3A-OE NPCs increased their DNA ratio versus CNTL at days 11 and 28 and conversely, WNT11-OE significantly decreased DNA content at these same time points (Fig. 4C).

\section{CRISPR-Cas9 lentiviral transduction knocked out WNT genes}

After viral transduction of NPCs with CRISPR-Cas9 vectors, guided by sgRNA sequences targeting WNT3A, WNT5A, and WNT11 exons, selection was conducted via puromycin supplementation for the knockout (KO) groups. Three different lentiviral vectors designed in this study for each WNT gene, targeting different exon sequences, were compared for the most effective KO effect in NPCs. For the selected WNT-KO groups (WNT3A-1, WNT5A-3, and WNT11-2), each respective KO target was found most significantly diminished in both expanded cells and redifferentiation induced pellets compared to CNTL throughout the study. After determining WNT3A expression was significantly decreased (nondetectable, ND) in WNT-KO versus CNTL in NPCs following transduction in cell samples and redifferentiation pellets (Fig. 5A). WNT5A-KO expressed a nearly 10- to 40-fold decrease of WNT5A mRNA versus CNTL at each time point during redifferentiation (Fig. 5B). Similarly, WNT11-KO exhibited 5-fold or greater decrease of WNT11 expression compared to CNTL at day 0, day 11, and day 28 of redifferentiation induction (Fig. 5C).

Knockout of noncanonical WNT signals decreased redifferentiation capacity

All WNT-KO groups exhibited increased COL1A1 expression in cell samples following expansion (Fig. 6A). WNT5A-KO and WNT11-KO most significantly increased COL1A1 mRNA expression at day 0 versus CNTL; however, this expression was significantly attenuated at day 11 of redifferentiation but not in WNT3A-KO (Fig. 6A). Although the knockout of each respective WNT gene led to significant decreases in PAX1 (Fig. 6B) and FOXF1 (Fig. 6C) expression in all groups 
except an increase of PAX1 in WNT5A-KO at day 11 (Fig. 6B), COL2A1 expression was significantly decreased versus CNTL in both WNT5A-KO and WNT11-KO groups at cell samples and day 28 pellets, but not with the knockout of WNT3A gene (Fig. 6D). A similar trend was observed in ACAN expression, with both WNT5A-KO and WNT11-KO most significantly (approximately 5-fold) decreased versus CTNL (Fig. 6E).

Both WNT5A-KO and WNT11-KO groups exhibited a small size of pellets compared to CNTL (Fig. 7A). This finding was corroborated with biochemical analysis data, in which both WNT5AKO and WNT11-KO groups displayed a dramatically decreased GAG/DNA ratios (Fig. 7B) and GAG amounts per pellet (Fig. 7C) as well as lower DNA ratios except no significant difference of WNT11-KO versus CNTL at day 28 (Fig. 7D). Intriguingly, the WNT3A-KO group exhibited a significantly decrease of GAG/DNA ratio at day 28 rather than day 11 (Fig. 7B); however, both GAG amount per pellet (Fig. 7C) and DNA ratio (Fig. 7D) of the WNT3A-KO group had no significant difference compared to CNTL. 


\section{Discussion}

Although current literature has suggested roles of Wnt signaling in the intervertebral disc, few studies have determined roles for specific Wnt signals, particularly the potential roles of Wnt3A in NPC redifferentiation. In this study, we sought to directly compare the expression of specific Wnt signals to the redifferentiation of NPCs through lentiviral-mediated transduction. Following transduction, the results of this study conclude that the virally-mediated overexpression or knockout of noncanonical Wnt signals, Wnt5A and Wnt11, were much more influential in their collective impact on the redifferentiation gene expression than the effect of CRISPR-Cas9mediated knockout of canonical (WNT3A) expression in human NPCs. To our knowledge, this study is the first in directly comparing the influences of WNT3A, WNT5A, and WNT11 gene expression in the redifferentiation of human NPCs.

We found that WNT3A-OE was the only group during WNT-gene overexpression that expressed any detectable WNT3A mRNA at each timepoint. In previous preliminary experiments, WNT3A gene expression was consistently lower than other wnt signals, or usually undetectable by qPCR (not shown). This result was similarly reported by Smolders et al., who reported in a degenerative disc study that "gene expression of the Wnt3a was undetectable in all groups" (Smolders et al., 2013). Despite generally less robust expression when compared to noncanonical signals, in this study, WNT3A overexpression increased both proliferation index and peak generation number compared to other groups and was the only group to significantly increase DNA content during redifferentiation. These results seem to be consistent with other reports. Narcisi and colleagues, reported that Wnt3A has increased proliferation in MSCs (Narcisi et al., 2015), and similar results have been reported in which Wnt3A has been shown to promote the proliferation of pancreatic 
stem cells (He et al., 2015), neural stem cells (Yang et al., 2014), bone sarcoma cells (Chen et al., 2015), and HEK293T cells (Reischmann et al., 2015).

As expected, the knockout of all WNT genes led to significantly decreased PAX1 and FOXF1 expression, markers suggested to be representative of the healthy, mature nucleus pulposus phenotype (Minogue et al., 2010; Thorpe et al., 2016). Interestingly, the overexpression of WNT5A and WNT11 genes increased PAX1 expression by five-fold and nine-fold, respectively, versus control at day 0 of redifferentiation; however, this effect was attenuated by day 28 . Similarly, WNT11-OE was the only group to significantly increase FOXF1 expression (6.5-fold) at the day 0 time point, while the other groups were significantly decreased versus CNTL, further suggesting WNT11's role as an early regulator of NPC redifferentiation. At day 11, noncanonical WNT overexpression (WNT5A-OE and WNT11-OE) were both significantly increased versus CNTL for FOXF1 expression, but not WNT3A-OE. By day 28, these effects were abolished, and in fact, significantly decreased for noncanonical signal overexpression groups. Overall, as expected, WNT5A-OE and WNT11-OE produced more dramatic changes in ACAN mRNA versus CNTL than WNT3A-OE in early to middle-stage of redifferentiation.

Cumulative trends in these redifferentiation data seem to suggest that, early in redifferentiation, noncanonical signals can increase markers representative of mature NPCs. This trend does not remain consistent through day 28 , however, and is likely due to the persistent overexpression of these Wnt signals, as they are more tightly regulated to increase and decrease appropriately in nontransduced cells. This same early to middle-stage expression trend is apparent in COL2A1 expression of WNT-overexpressing NPCs, particularly notable for WNT5A and WNT11 signals. WNT11-OE seems most influential in early NPC redifferentiation. This notion of stage-specific Wnt regulation of differentiation is supported by evidence from a study by Sinha and colleagues, 
where they implicated WNT11 as an early expression gene in tissue development and differentiation (Sinha et al., 2015). In another study investigating mouse limb development, both Wnt5A and Wnt11 were implicated in early limb development and chondrogenesis, whereas Wnt3A was undetectable throughout (Witte et al., 2009). Another recent study investigating the role of miRNA-410 in MSCs found that miRNA-410, a promoter of chondrogenesis, slightly increased at day 15 of differentiation and significantly elevated at day 25 . They report that this miRNA also pairs with Wnt3A mRNA and that increases in miRNA-410 negatively correlates with Wnt3A protein expression (Zhang et al., 2017b). Further studies are needed to investigate the stage-specific expression of Wnt signals and Wnt agonists/regulators, and could provide more detailed evidence for Wnt regulation in differentiation.

Despite the positive results in redifferentiation gene expression, we recognize the limitations of this study. It remains to be determined whether WNT5A or WNT11 are individually superior to one another or to combination gene therapy, which was not clearly distinguishable in our results. Future studies which more thoroughly evaluate other downstream targets of both canonical and noncanonical Wnt signals, such as axin, cyclin D1, APC, and $\beta$-catenin could provide further evidence to support the findings of this study and in the redifferentiation of human nucleus pulposus cells. Furthermore, a study which uses both overexpression and targeted knockout of WNT genes at various stages of differentiation (early, middle, and late) in the same NPCs could more accurately and completely elucidate the stage-specificity of Wnt signals in NPC redifferentiation, rather than the relatively static methods of genetic manipulation used in our study. This design is especially important when consideration is given to the natural expression patterns of both canonical and noncanonical Wnt signals during differentiation. 
Although many studies aim to highlight the apparent differences between canonical and noncanonical Wnt signals for cell differentiation, it is important to recognize the interplay, crosstalk, and potential advantageous utilization of each of the Wnt signaling pathways. Interestingly, Hsu and Huang reported that Wnt5A-mediated and Wnt3A-mediated MSC differentiation could each be directed based on distinct, three-dimensional substrate and culture environments (Hsu \& Huang, 2013), and raises new concerns for NPC expansion and intervertebral disc tissue engineering. A recent study by Alok and colleagues has suggested that Wnt signals, particularly those of the noncanonical variety, can synergistically activate $\beta$-catenin in several cell types as a combinatory mechanism regulating wnt signaling (Alok et al., 2017). It is possible that noncanonical signals in this study, WNT5A and WNT11, can work together in a similar, potentially stage-specific manner during redifferentiation of nucleus pulposus cells. This concept of stage-specific expression has been recently suggested in human SDSCs during their expansion in the presence of fibroblast growth factor 2 or chondrogenic differentiation (Pizzute et al., 2016). Another recent study has provided evidence that several noncanonical signals in the liver, including WNT5A, can act as antagonists to Wnt3A-induced $\beta$-catenin/TCF signaling to promote tissue differentiation, suggesting noncanonical signals can "fine-tune" the effects of canonical Wnt signaling (Fan et al., 2017). Additionally, studies that can uncover further regulatory intricacies and the crosstalk between canonical and noncanonical wnt pathways would be beneficial; for example, recent studies have demonstrated canonical suppression can occur through PTK7 (Berger et al., 2017), as well as crosstalk with the Smad/TGF-Beta pathway (Webber et al., 2016), known to promote differentiation. Overall, it seems likely that both canonical and noncanonical Wnt signals are significantly involved in the redifferentiation of human NPCs; however, based on our results, it seems that WNT3A can potentially contribute to 
NPC proliferation, whereas noncanonical signals promote early redifferentiation. In conclusion, changes in Wnt signal expression can modulate the redifferentiation of human NPCs. It may be most effective to promote noncanonical Wnt pathway activation through WNT5A and WNT11 than canonical WNT3A. Each signal represents potential targets for NPC redifferentiation and for future therapeutic strategies aiming to improve NP tissue regeneration. 


\section{Acknowledgements}

We thank Suzanne Danley for editing the manuscript. This project was partially supported by

Research Grants from the Musculoskeletal Transplant Foundation and the National Institutes of Health (R03AR062763-01A1, R01AR067747-01A1). 


\section{Author Disclosure Statement}

No competing financial interests exist. 


\section{References}

1. Alok A, Lei Z, Jagannathan NS, Kaur S, Rozen SG, Tucker-Kellogg L, Virshup DM. Wnts synergize to activate $\beta$-catenin signaling. J Cell Sci. 2017 Mar 13.

2. Arai F, Hiyama A, Sakai D, Yokoyama K, Mochida J. The expression and role of noncanonical (PKC) signaling in nucleus pulposus cell metabolism. J Orthop Res. 2012 Sep;30(9):1478-85.

3. Bowles RD, Setton LA. Biomaterials for intervertebral disc regeneration and repair. Biomaterials. 2017 Jun;129:54-67.

4. Buchtova M, Oralova V, Aklian A, Masek J, Vesela I, Ouyang Z, Obadalova T, Konecna Z, Spoustova T, Pospisilova T, Matula P, Varecha M, Balek L, Gudernova I, Jelinkova I, Duran I, Cervenkova I, Murakami S, Kozubik A, Dvorak P, Bryja V, Krejci P. Fibroblast growth factor and canonical WNT/ $\beta$-catenin signaling cooperate in suppression of chondrocyte differentiation in experimental models of FGFR signaling in cartilage. Biochim Biophys Acta. 2015 May;1852(5):839-50.

5. Cai F, Zhu L, Wang F, Shi R, Xie XH, Hong X, Wang XH, Wu XT. The Paracrine Effect of Degenerated Disc Cells on Healthy Human Nucleus Pulposus Cells Is Mediated by MAPK and

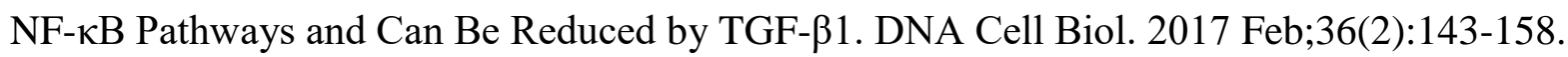

6. Chen C, Zhao M, Tian A, Zhang X, Yao Z, Ma X. Aberrant activation of Wnt $/ \beta$-catenin signaling drives proliferation of bone sarcoma cells. Oncotarget. 2015 Jul 10;6(19):17570-83.

7. Chen S, Fu P, Wu H, Pei M. Meniscus, articular cartilage and nucleus pulposus: a comparative review of cartilage-like tissues in anatomy, development and function. Cell Tissue Res. 2017 Apr 17.

8. Chen WH, Lo WC, Lee JJ, Su CH, Lin CT, Liu HY, Lin TW, Lin WC, Huang TY, Deng WP. Tissue-engineered intervertebral disc and chondrogenesis using human nucleus pulposus regulated through TGF-beta1 in platelet-rich plasma. J Cell Physiol. 2006 Dec;209(3):744-54.

9. Cleary MA, van Osch GJ, Brama PA, Hellingman CA, Narcisi R. FGF, TGF $\beta$ and Wnt 
crosstalk: embryonic to in vitro cartilage development from mesenchymal stem cells. J Tissue Eng Regen Med. 2015 Apr;9(4):332-42.

10. Dahia CL, Mahoney EJ, Durrani AA, Wylie C. Intercellular signaling pathways active during intervertebral disc growth, differentiation, and aging. Spine (Phila Pa 1976). 2009 Mar 1;34(5):456-62.

11. Dieleman JL, Baral R, Birger M, Bui AL, Bulchis A, Chapin A, Hamavid H, Horst C, Johnson EK, Joseph J, Lavado R, Lomsadze L, Reynolds A, Squires E, Campbell M, DeCenso B, Dicker D, Flaxman AD, Gabert R, Highfill T, Naghavi M, Nightingale N, et al. US Spending on Personal Health Care and Public Health, 1996-2013. JAMA. 2016 Dec 27;316(24):26272646.

12. Fan J, Wei Q, Liao J, Zou Y, Song D, Xiong D, Ma C, Hu X, Qu X, Chen L, Li L, Yu Y, Yu X, Zhang Z, Zhao C, Zeng Z, Zhang R, Yan S, Wu T, Wu X, Shu Y, Lei J, et al. Noncanonical Wnt signaling plays an important role in modulating canonical Wnt-regulated stemness, proliferation and terminal differentiation of hepatic progenitors. Oncotarget. 2017 Feb 23.

13. Garcin CL, Habib SJ. A Comparative Perspective on Wnt/ $\beta$-Catenin Signalling in Cell Fate Determination. Results Probl Cell Differ. 2017;61:323-350.

14. Green JD, Tollemar V, Dougherty M, Yan Z, Yin L, Ye J, Collier Z, Mohammed MK, Haydon RC, Luu HH, Kang R, Lee MJ, Ho SH, He TC, Shi LL, Athiviraham A. Multifaceted signaling regulators of chondrogenesis: Implications in cartilage regeneration and tissue engineering. Genes Dis. 2015 Dec;2(4):307-327.

15. He X, Han W, Hu SX, Zhang MZ, Hua JL, Peng S. Canonical Wnt signaling pathway contributes to the proliferation and survival in porcine pancreatic stem cells (PSCs). Cell Tissue Res. 2015 Nov;362(2):379-88.

16. Hegewald AA, Endres M, Abbushi A, Cabraja M, Woiciechowsky C, Schmieder K, Kaps C, Thomé C. Adequacy of herniated disc tissue as a cell source for nucleus pulposus regeneration. $\mathbf{J}$ Neurosurg Spine. 2011 Feb;14(2):273-80. 
17. Hiyama A, Yokoyama K, Nukaga T, Sakai D, Mochida J. A complex interaction between Wnt signaling and TNF- $\alpha$ in nucleus pulposus cells. Arthritis Res Ther. 2013 Nov 14;15(6):R189.

18. Hiyama A, Sakai D, Tanaka M, Arai F, Nakajima D, Abe K, Mochida J. The relationship between the Wnt/ $\beta$-catenin and TGF- $\beta /$ BMP signals in the intervertebral disc cell. J Cell Physiol. 2011 May;226(5):1139-48.

19. Hiyama A, Sakai D, Risbud MV, Tanaka M, Arai F, Abe K, Mochida J. Enhancement of intervertebral disc cell senescence by WNT/ $\beta$-catenin signaling-induced matrix metalloproteinase expression. Arthritis Rheum. 2010 Oct;62(10):3036-47.

20. Hosseini-Farahabadi S, Geetha-Loganathan P, Fu K, Nimmagadda S, Yang HJ, Richman JM. Dual functions for WNT5A during cartilage development and in disease. Matrix Biol. 2013 Jun 24;32(5):252-64.

21. Hsu SH, Huang GS. Substrate-dependent Wnt signaling in MSC differentiation within biomaterial-derived 3D spheroids. Biomaterials. 2013 Jul;34(20):4725-38.

22. Jia H, Ma J, Lv J, Ma X, Xu W, Yang Y, Tian A, Wang Y, Sun L, Xu L, Fu L, Zhao J. Oestrogen and parathyroid hormone alleviate lumbar intervertebral disc degeneration in ovariectomized rats and enhance Wnt/ $\beta$-catenin pathway activity. Sci Rep. 2016 Jun 9;6:27521.

23. Kondo N, Yuasa T, Shimono K, Tung W, Okabe T, Yasuhara R, Pacifici M, Zhang Y, Iwamoto M, Enomoto-Iwamoto M. Intervertebral disc development is regulated by $\mathrm{Wnt} / \beta$ catenin signaling. Spine (Phila Pa 1976). 2011 Apr 15;36(8):E513-8.

24. Lee HC, Lim S, Han JY. Wnt/ $/$-catenin signaling pathway activation is required for proliferation of chicken primordial germ cells in vitro. Sci Rep. 2016 Sep 30;6:34510.

25. Liu S, Zhang E, Yang M, Lu L. Overexpression of Wnt11 promotes chondrogenic differentiation of bone marrow-derived mesenchymal stem cells in synergism with TGF- $\beta$. Mol Cell Biochem. 2014 May;390(1-2):123-31. 
26. Matta A, Karim MZ, Isenman DE, Erwin WM. Molecular Therapy for Degenerative Disc Disease: Clues from Secretome Analysis of the Notochordal Cell-Rich Nucleus Pulposus. Sci Rep. 2017 Mar 30;7:45623.

27. McCann MR, Séguin CA. Notochord Cells in Intervertebral Disc Development and Degeneration. J Dev Biol. 2016 Jan 21;4(1):1-18

28. Meng X, Baylink DJ, Sheng M, Wang H, Gridley DS, Lau KH, Zhang XB. Erythroid promoter confines FGF2 expression to the marrow after hematopoietic stem cell gene therapy and leads to enhanced endosteal bone formation. PLoS One. 2012;7(5):e37569.

29. Minogue BM, Richardson SM, Zeef LA, Freemont AJ, Hoyland JA. Characterization of the human nucleus pulposus cell phenotype and evaluation of novel marker gene expression to define adult stem cell differentiation. Arthritis Rheum. 2010 Dec;62(12):3695-705.

30. Mohammed MK, Shao C, Wang J, Wei Q, Wang X, Collier Z, Tang S, Liu H, Zhang F, Huang J, Guo D, Lu M, Liu F, Liu J, Ma C, Shi LL, Athiviraham A, He TC, Lee MJ. Wnt/Bcatenin signaling plays an ever-expanding role in stem cell self-renewal, tumorigenesis and cancer chemoresistance. Genes Dis. 2016 Mar;3(1):11-40.

32. Narcisi R, Arikan OH, Lehmann J, Ten Berge D, van Osch GJ. Differential Effects of Small Molecule WNT Agonists on the Multilineage Differentiation Capacity of Human Mesenchymal Stem Cells. Tissue Eng Part A. 2016 Nov;22(21-22):1264-1273. E

33. Narcisi R, Cleary MA, Brama PA, Hoogduijn MJ, Tüysüz N, ten Berge D, van Osch GJ. Long-term expansion, enhanced chondrogenic potential, and suppression of endochondral ossification of adult human MSCs via WNT signaling modulation. Stem Cell Reports. 2015 Mar 10;4(3):459-72. doi: 10.1016/j.stemcr.2015.01.017.

34. Pei M. Environmental preconditioning rejuvenates adult stem cells' proliferation and chondrogenic potential. Biomaterials. 2017 Feb;117:10-23. 
35. Shao J, Yu M, Jiang L, Wu F, Liu X. Sequencing and bioinformatics analysis of the differentially expressed genes in herniated discs with or without calcification. Int J Mol Med. 2017 Jan;39(1):81-90.

36. Shoukry M, Li J, Pei M. Reconstruction of an in vitro niche for the transition from intervertebral disc development to nucleus pulposus regeneration. Stem Cells Dev. 2013 Apr 15;22(8):1162-76.

37. Sinha T, Lin L, Li D, Davis J, Evans S, Wynshaw-Boris A, Wang J. Mapping the dynamic expression of Wnt11 and the lineage contribution of Wnt11-expressing cells during early mouse development. Dev Biol. 2015 Feb 15;398(2):177-92.

38. Smolders LA, Meij BP, Onis D, Riemers FM, Bergknut N, Wubbolts R, Grinwis GC, Houweling M, Groot Koerkamp MJ, van Leenen D, Holstege FC, Hazewinkel HA, Creemers LB, Penning LC, Tryfonidou MA. Gene expression profiling of early intervertebral disc degeneration reveals a down-regulation of canonical Wnt signaling and caveolin-1 expression: implications for development of regenerative strategies. Arthritis Res Ther. 2013 Jan 29;15(1):R23.

39. Srivastava A, Isa IL, Rooney P, Pandit A. Bioengineered three-dimensional diseased intervertebral disc model revealed inflammatory crosstalk. Biomaterials. 2017 Apr;123:127-141.

40. Thorfve A, Dehne T, Lindahl A, Brittberg M, Pruss A, Ringe J, Sittinger M, Karlsson C. Characteristic Markers of the WNT Signaling Pathways Are Differentially Expressed in Osteoarthritic Cartilage. Cartilage. 2012 Jan;3(1):43-57.

41. Thorpe AA, Binch AL, Creemers LB, Sammon C, Le Maitre CL. Nucleus pulposus phenotypic markers to determine stem cell differentiation: fact or fiction? Oncotarget. 2016 Jan 19;7(3):2189-200.

42. Twomey LT, Taylor JR. Age changes in lumbar vertebrae and intervertebral discs. Clin Orthop Relat Res. 1987 Nov;(224):97-104.

43. Urban JP, Roberts S. Development and degeneration of the intervertebral discs. 
Mol Med Today. 1995 Oct;1(7):329-35.

44. Usami Y, Gunawardena AT, Iwamoto M, Enomoto-Iwamoto M. Wnt signaling in cartilage development and diseases: lessons from animal studies. Lab Invest. 2016 Feb;96(2):186-96.

45. Wang J, Chen H, Cao P, Wu X, Zang F, Shi L, Liang L, Yuan W. Inflammatory cytokines induce caveolin-1/ $\beta$-catenin signalling in rat nucleus pulposus cell apoptosis through the $\mathrm{p} 38$ MAPK pathway. Cell Prolif. 2016 Jun;49(3):362-72.

46. Webber HC, Bermudez JY, Sethi A, Clark AF, Mao W. Crosstalk between TGF $\beta$ and Wnt signaling pathways in the human trabecular meshwork. Exp Eye Res. 2016 Jul;148:97-102.

47. Winkler T, Mahoney EJ, Sinner D, Wylie CC, Dahia CL. Wnt signaling activates Shh signaling in early postnatal intervertebral discs, and re-activates Shh signaling in old discs in the mouse. PLoS One. 2014;9(6):e98444.

48. Witte F, Dokas J, Neuendorf F, Mundlos S, Stricker S. Comprehensive expression analysis of all Wnt genes and their major secreted antagonists during mouse limb development and cartilage differentiation. Gene Expr Patterns. 2009 Apr;9(4):215-23.

49. Xie H, Jing Y, Xia J, Wang X, You C, Yan J. Aquaporin 3 protects against lumbar intervertebral disc degeneration via the Wnt/ $\beta$-catenin pathway. Int J Mol Med. 2016 Mar;37(3):859-64.

50. Yang XT, Bi YY, Chen ET, Feng DF. Overexpression of Wnt3a facilitates the proliferation and neural differentiation of neural stem cells in vitro and after transplantation into an injured rat retina. J Neurosci Res. 2014 Feb;92(2):148-61.

51. Zhang JP, Li XL, Li GH, Chen W, Arakaki C, Botimer GD, Baylink D, Zhang L, Wen W, Fu YW, Xu J, Chun N, Yuan W, Cheng T, Zhang XB. Efficient precise knockin with a double cut HDR donor after CRISPR/Cas9-mediated double-stranded DNA cleavage. Genome Biol. 2017a Feb 20;18(1):35. 
52. Zhang JP, Li XL, Neises A, Chen W, Hu LP, Ji GZ, Yu JY, Xu J, Yuan WP, Cheng T, Zhang XB. Different Effects of sgRNA Length on CRISPR-mediated Gene Knockout Efficiency. Sci Rep. 2016 Jun 24;6:28566.

53.. Zhang Y, Pizzute T, Pei M. A review of crosstalk between MAPK and Wnt signals and its impact on cartilage regeneration. Cell Tissue Res. 2014 Dec;358(3):633-49.

54. Zhang Y, Huang X, Yuan Y. MicroRNA-410 promotes chondrogenic differentiation of human bone marrow mesenchymal stem cells through down-regulating Wnt3a. Am J Transl Res. 2017b;9(1):136-145.

55. Zhong L, Huang X, Karperien M, Post JN. The Regulatory Role of Signaling Crosstalk in Hypertrophy of MSCs and Human Articular Chondrocytes. Int J Mol Sci. 2015 Aug 14;16(8):19225-47.

56. Zhu X, Zhu H, Zhang L, Huang S, Cao J, Ma G, Feng G, He L, Yang Y, Guo X. Wlsmediated Wnts differentially regulate distal limb patterning and tissue morphogenesis. Dev Biol. 2012 May 15;365(2):328-38. 
Figure 3.1
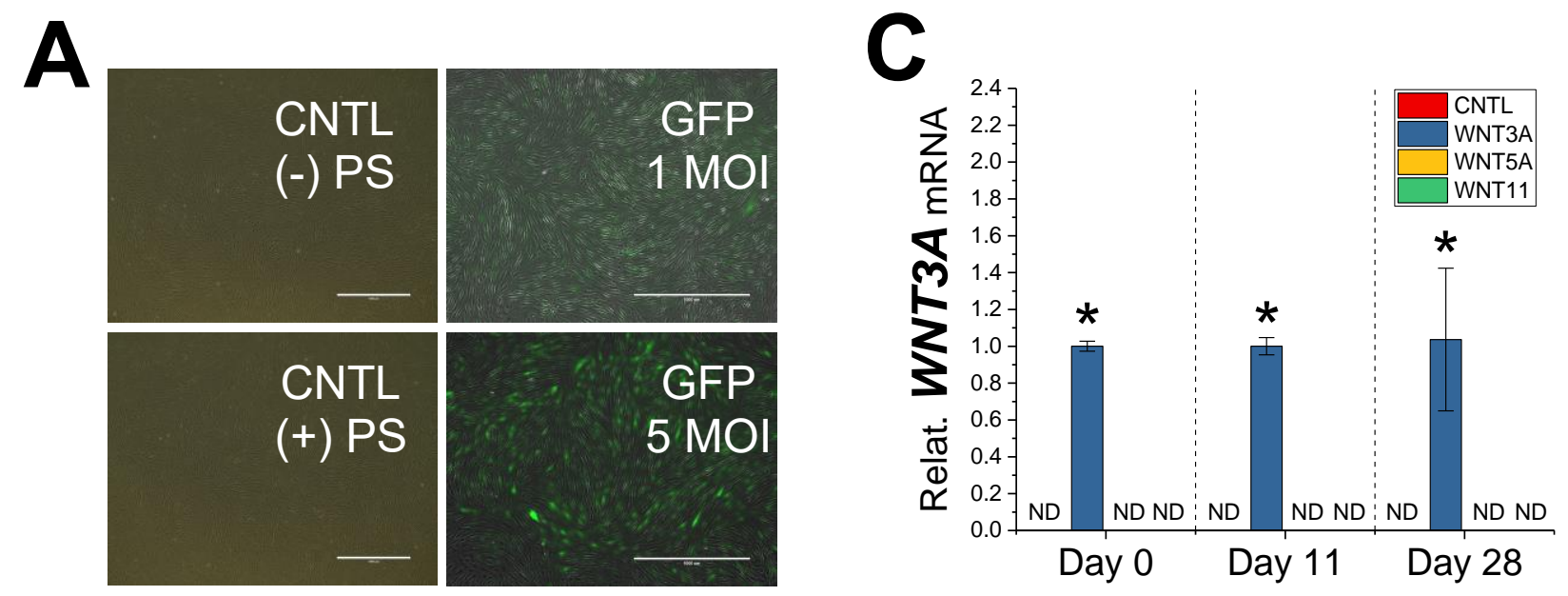

B
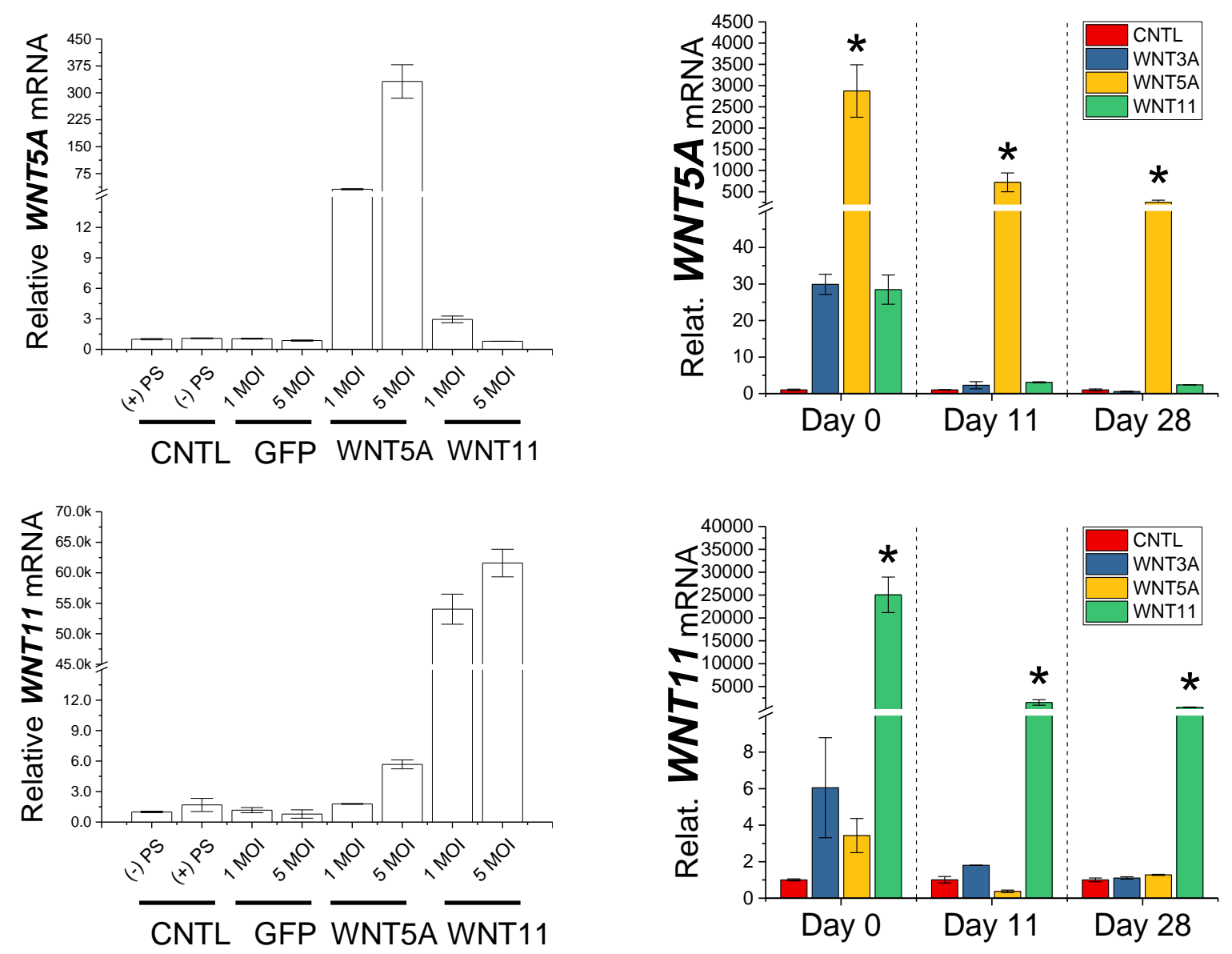
Figure 3.1: Overexpression of WNT genes during redifferentiation. Following transduction, GFP-expression was visualized by immunofluorescence (A) and by qPCR (B) in cell samples. After pellet formation, samples were analyzed at each timepoint (Day 0,11, \& 28) of redifferentiation via qPCR for WNT3A (A), WNT5A (B), and WNT11 (C) mRNA expression. 
Figure 3.2
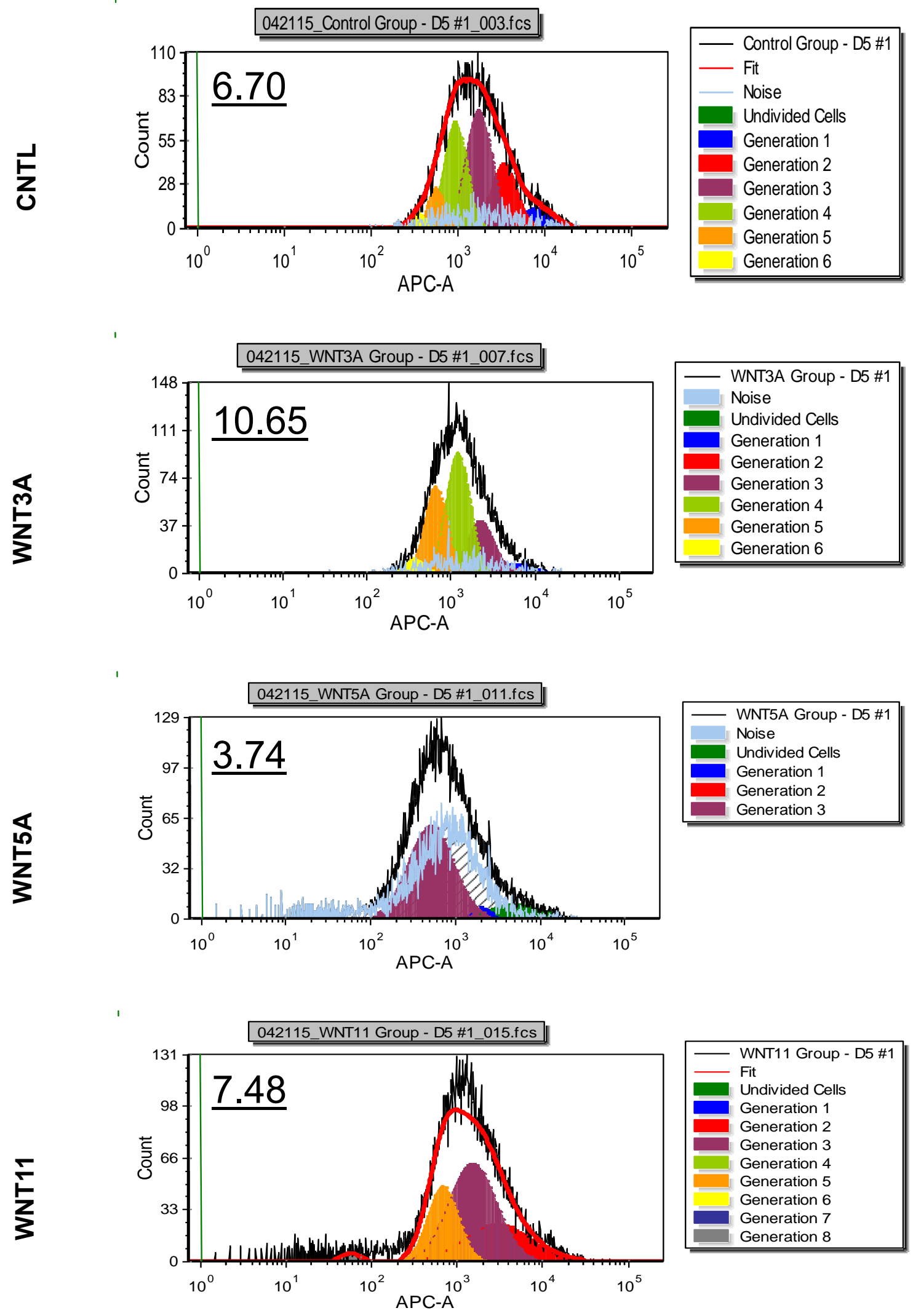
Figure 3.2: Proliferation of WNT-KO cells. Flow cytometry generational analysis of claret red proliferation for CNTL, WNT3A-KO, WNT5A-KO, and WNT11-KO after 5 days in culture. 
Figure 3.3

A
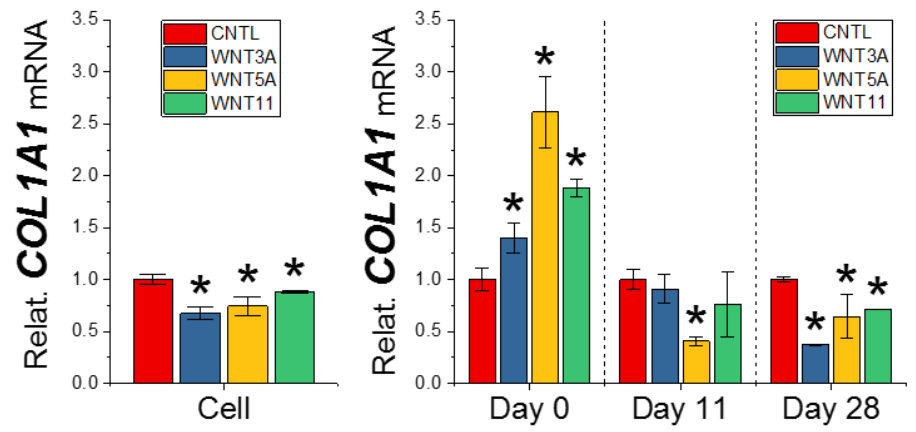

B
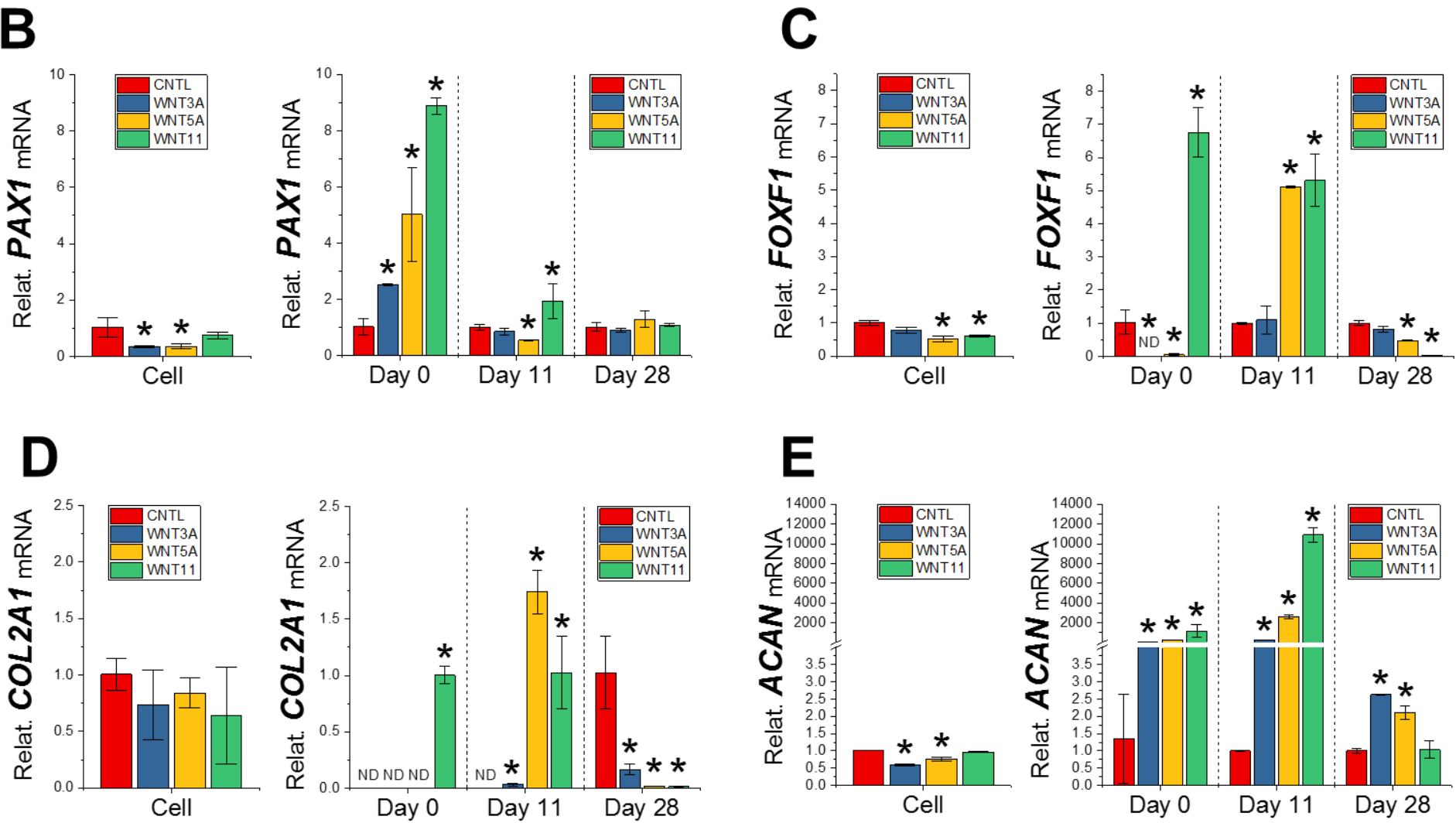
Figure 3.3 Redifferentiation gene expression in WNT-OE. Expression of COL1A1 (A), PAX1 (B), FOXF1 (C), COL2A1 (D), and ACAN (E) mRNA in pellets overexpressing WNT genes across each timepoint (Day $0,11, \& 28$ ) of redifferentiation. 
Figure 3.4

A CNTL WNT3A WNT5A WNT11

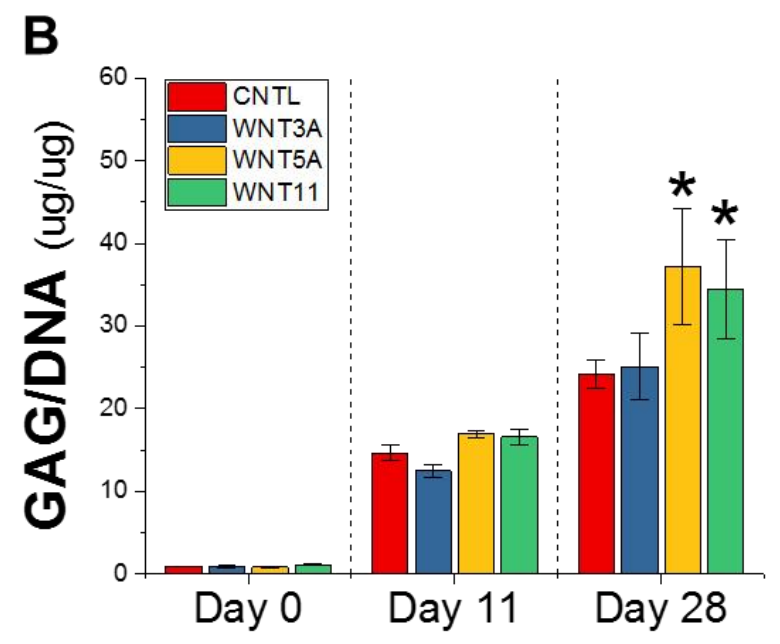

C
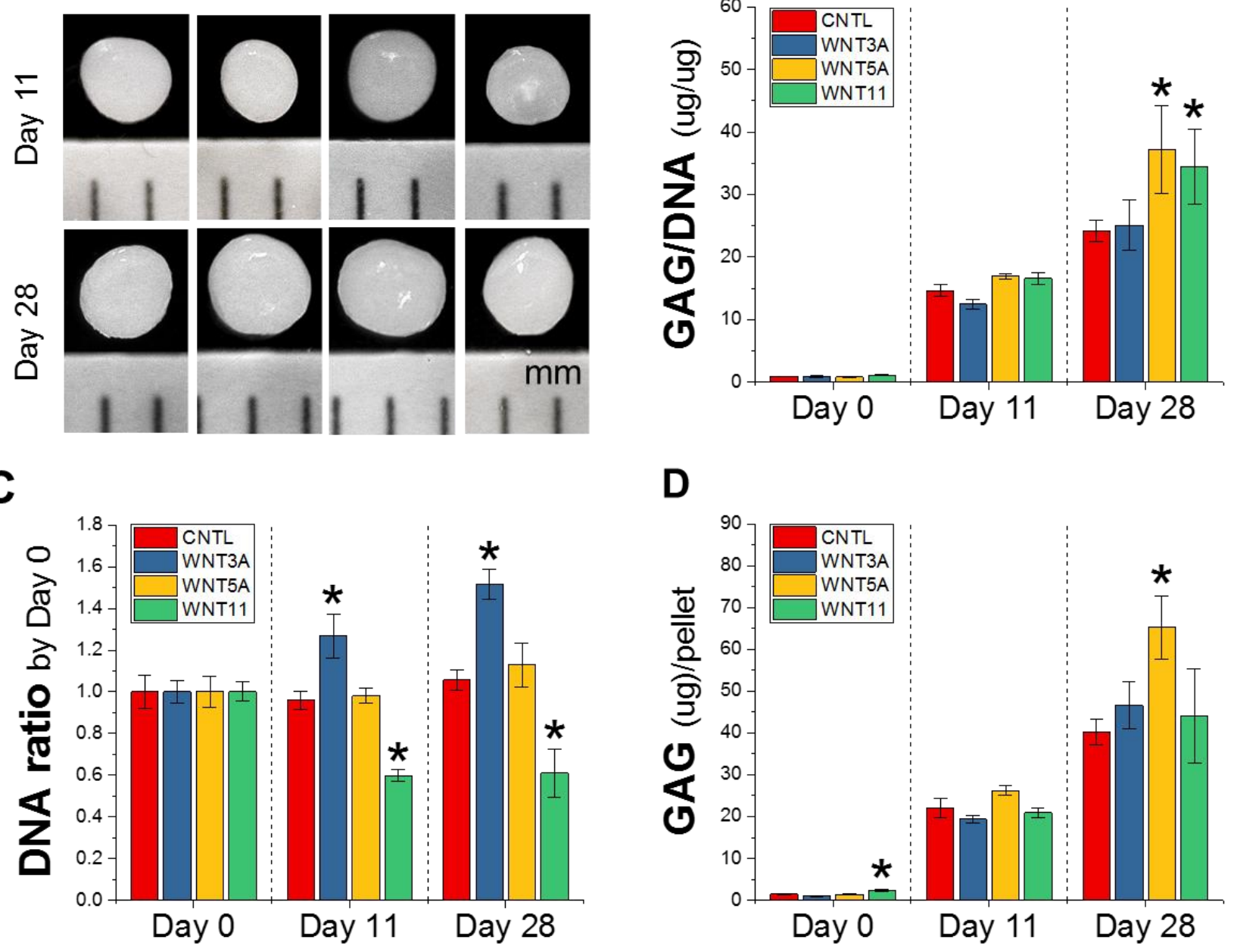
Figure 3.4: Redifferentiation of WNT-OE pellets. Representative pellets for each group at Day 11 and Day 28 of redifferentiation (A), GAG/DNA (B), DNA ratio (C), and GAG content (D) were analyzed. 
Figure 3.5

A
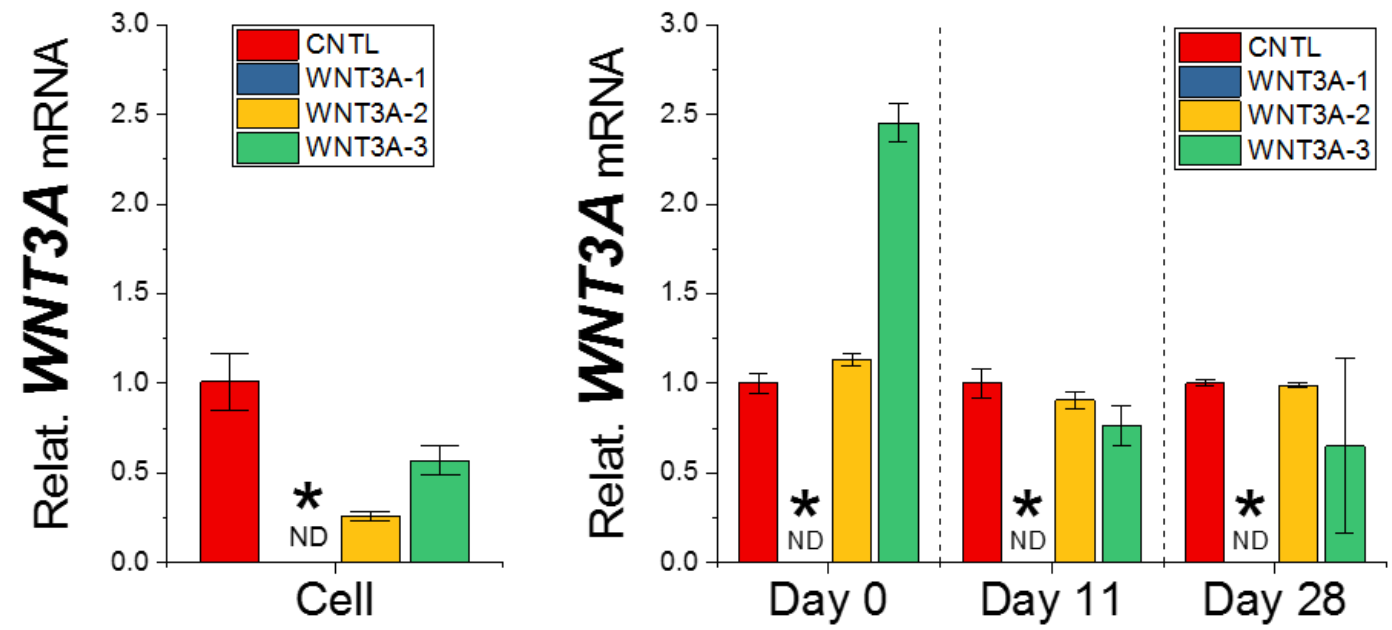

B
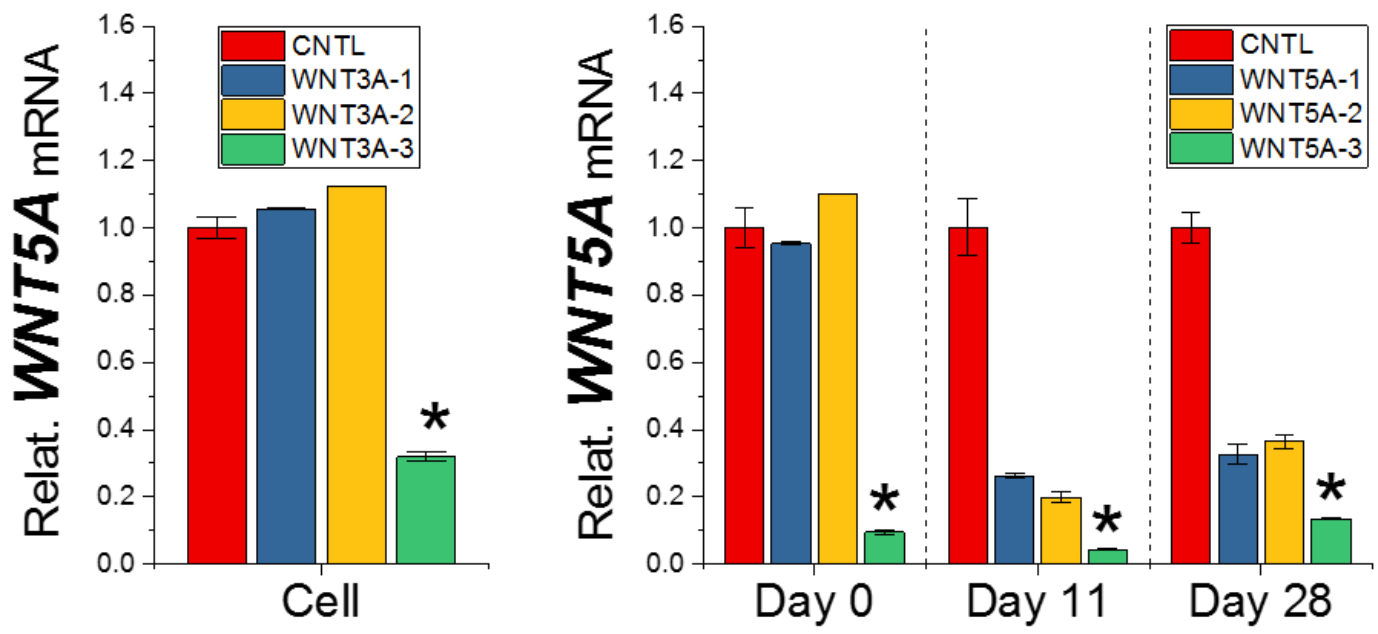

C
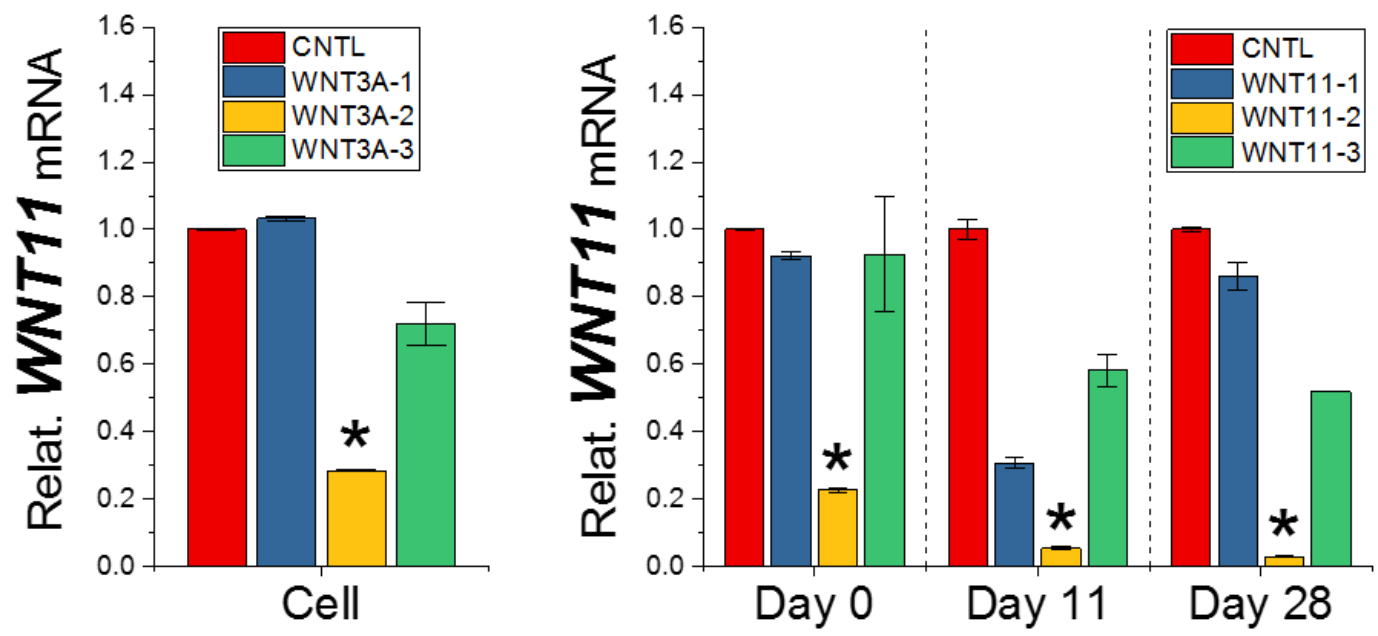
Figure 3.5 Knockout of WNT genes. Following transduction, cell expansion, and pellet formation, samples were analyzed in cell samples and at each timepoint (Day $0,11, \& 28$ ) of redifferentiation for WNT3A (A), WNT5A (B), and WNT11 (C) mRNA expression for each set of respective knockout vectors. 
Figure 3.6

A
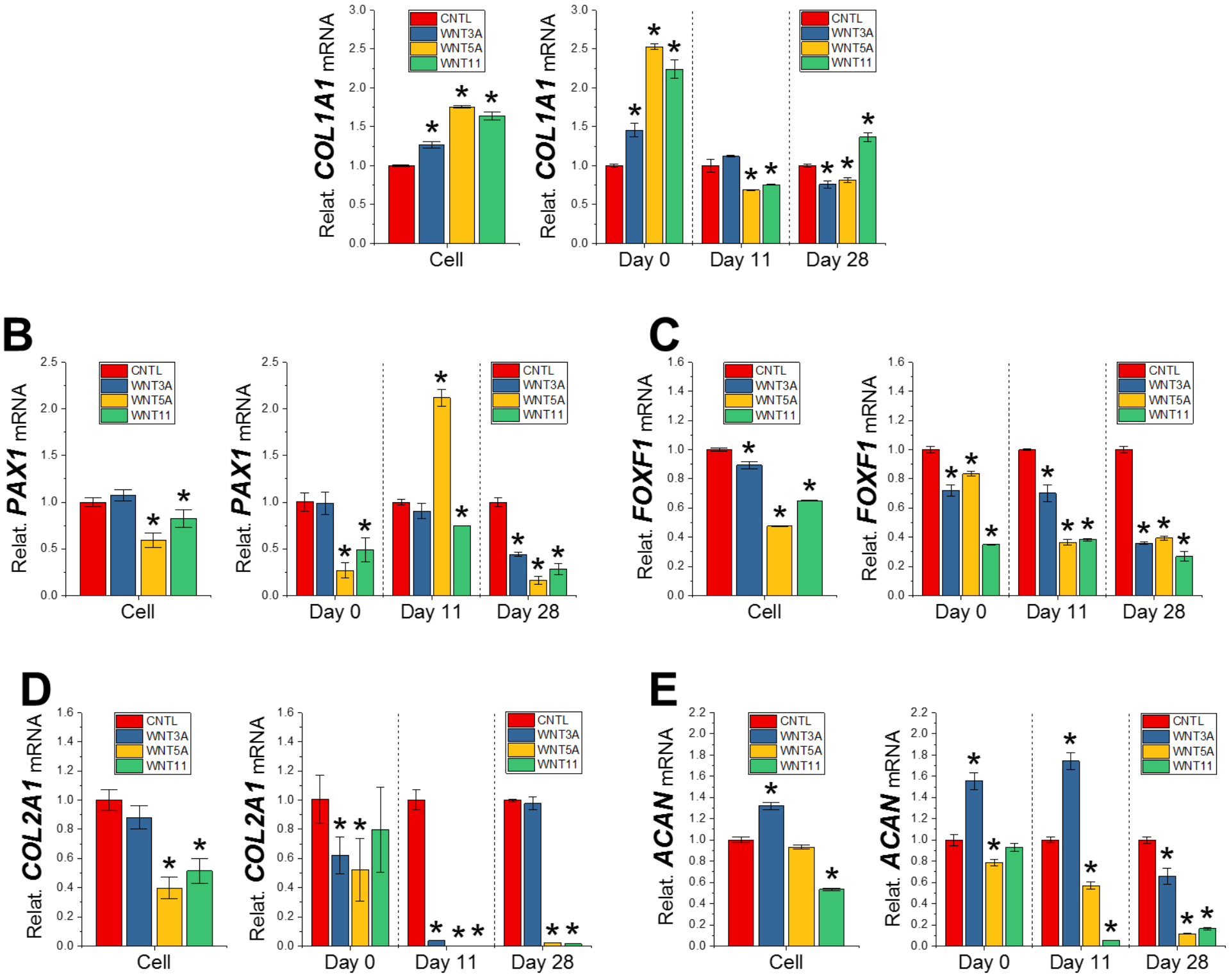
Figure 3.6: Redifferentiation gene expression in WNT-KO. Expression of COL1A1 (A), PAX1 (B), FOXF1 (C), COL2A1 (D), and ACAN (E) mRNA in pellets knocked out for WNT genes across each timepoint (Day $0,11, \& 28$ ) of redifferentiation. 
Figure 3.7

A CNTL WNT3A WNT5A WNT11

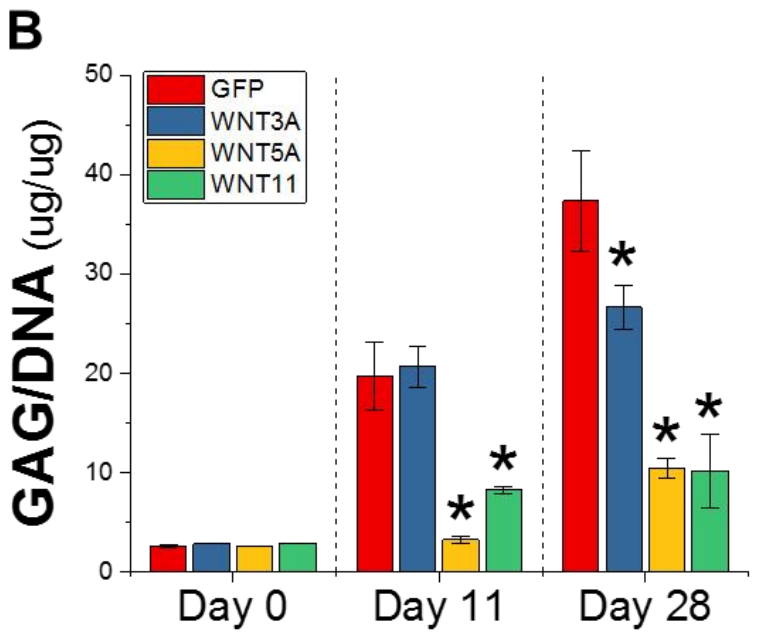

C

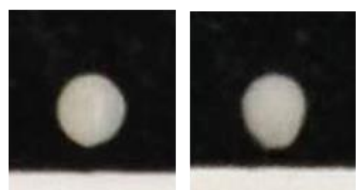

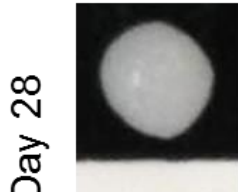
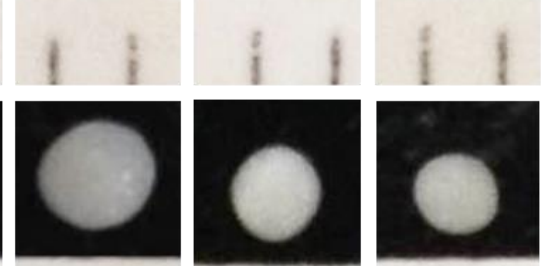

จิ
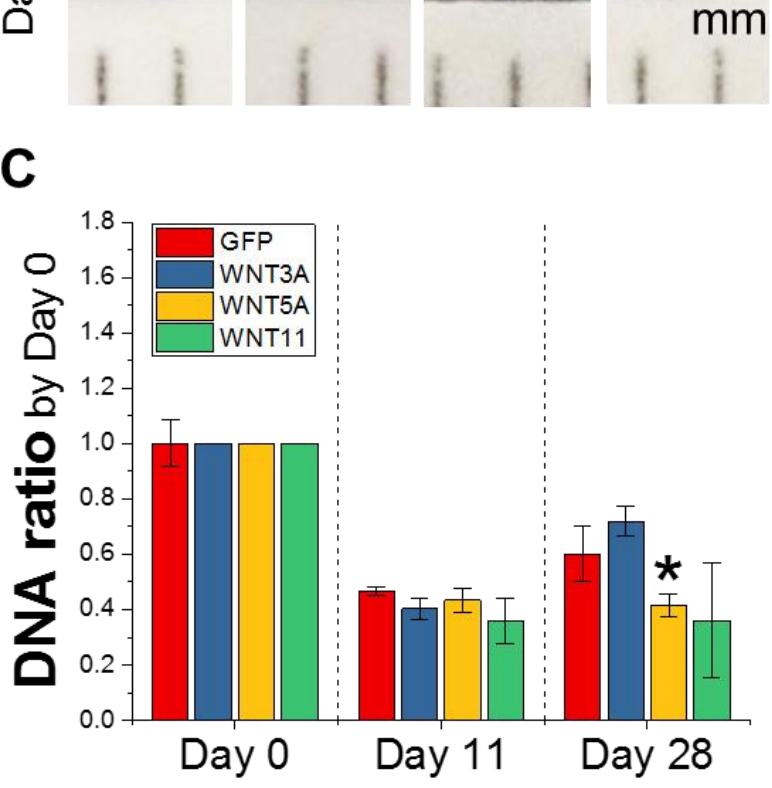

D

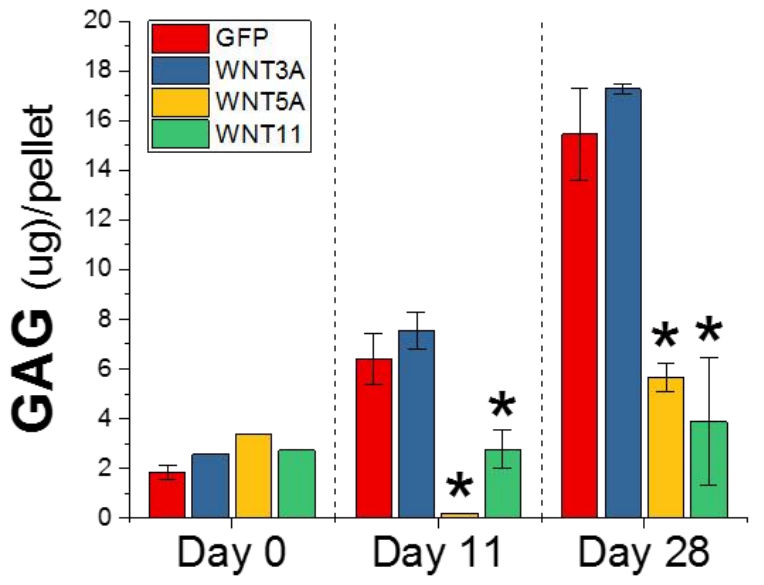


Figure 3.7: Redifferentiation of WNT-KO pellets. Representative pellets for each group at Day 11 and Day 28 of redifferentiation (A), GAG/DNA (B), DNA ratio (C), and GAG content (D) were analyzed. 


\section{Chapter 4:}

\section{Preconditioning strategies unequally impact}

\section{regeneration of nucleus pulposus cells from human}

\section{herniated discs: a proof of principle study}

As submitted (under review); TBD 2017

Tyler T. Pizzute, Sanford Emery, Ying Zhang, Shanawar Waris, Ming Pei.

Stem Cell and Tissue Engineering Laboratory, Department of Orthopaedics, West Virginia University, One Medical Center Drive, PO Box 9196, Morgantown, WV 26506-9196, USA.

Running Title: Preconditioning Nucleus Pulposus Immunity

Corresponding author: Ming Pei, MD, PhD

Stem Cell and Tissue Engineering Laboratory, Department of Orthopaedics, West Virginia University, PO Box 9196, One Medical Center Drive, Morgantown, WV 26506-9196, USA, Telephone: 304-293-1072; Fax: 304-293-7070; Email: mpei@hsc.wvu.edu 


\begin{abstract}
BACKGROUND: After herniation occurs, the intervertebral disc begins a progressive degenerative process where the inner nucleus pulposus (NP) tissue is compromised, failing to regenerate on its own if left untreated. Current treatment strategies typically focus on pain management rather than regeneration of the degenerated discs.
\end{abstract}

PURPOSE: An attractive strategy for disc regeneration and repair is autologous NP cell expansion and ultimately tissue repair, for which, unfortunately, replicative senescence is a significant hurdle. Recent studies indicate that preconditioning strategies, including pretreatment with fibroblast growth factor 2 (FGF2) supplementation, hypoxia (HYPO), or decellularized extracellular matrix (DECM), can promote expanded cell based tissue regeneration. However, it is unknown whether variability exists in NP cells from herniated disc donors in response to varied preconditioning strategies. This study sought to determine the impact of preconditioning strategies on NP cells. We also collected preliminary data for determining the correlation between the severity of disc degeneration (clinical data) and potential NP regeneration (biological performance).

STUDY DESIGN: This study is a laboratory investigation.

METHODS: NP cells were harvested from two representative herniated disc patients with different degenerative severity. The cells subjected to preconditioning treatments including FGF2 supplementation, HYPO, DECM expansion, or plastic expansion were evaluated and their proliferation and redifferentiation potentials were compared.

RESULTS: NP cells harvested from donors with both moderately degenerated and severely degenerated discs increased their proliferative potential and decreased their CD95 expression in response to both FGF2 and DECM preconditioning. Donor cells from moderately degenerated 
discs increased redifferentiation potential with FGF2 and DECM treatments characterized by increased glycosaminoglycan (GAG) deposition, pellet size, and redifferentiation gene expression following redifferentiation induction. Interestingly, large discrepancies were found in donor disc cells upon FGF2 preconditioning between the two grades of degeneration.

CONCLUSIONS: Our results suggest that specific preconditioning strategies unequally impact redifferentiation of NP cells from human herniated discs. We also found that NP cells from moderately degenerated discs had a better redifferentiation capacity than those from severely degenerated discs in response to preconditioning treatment. This finding raises new questions that need to be further validated with large sample size. 


\section{Introduction}

Low back pain represents a significant worldwide health and financial burden and its occurrence is projected to substantially increase over the next several decades [1]. In fact, musculoskeletal pain has been reported to affect one in four adults and is the most common cause of physical disability and long-term pain [2]. Nucleus pulposus (NP) tissue, located at the innermost space of each intervertebral disc, is composed of NP cells surrounded by their proteoglycan and collagen rich, well-hydrated extracellular matrix (ECM). In addition to NP's functional responsibilities as part of the disc anatomy, including compressive force resistance and shock absorption, the NP ECM helps to nourish NP cells and maintain disc homeostasis $[3,4]$. When NP ECM is lost due to disc herniation, aging, or degeneration, the disc can be significantly compromised. Once degeneration occurs, it is difficult to regenerate NP tissue [5], in part due to the slow turnover rates of some major NP matrix macromolecules which further compromises the disc's structural integrity [4].

In order to combat the problems surrounding degenerative disc disease and herniation repairs, autologous NP cell-based therapies have gained increasing attention [6,7]. Hegewald and colleagues compared cells from the NP compartment with herniated disc tissue and found that the cells from herniated disc tissue demonstrated a weaker redifferentiation process in a threedimensional (3D) culture system [8]. By culturing NP cells harvested from the patient, in vitro expansion strategies can produce a large number of cells for therapeutic applications; however, these strategies present their own set of challenges, such as replicative senescence [9] and inflammation [10]. Therefore, rejuvenation of herniated disc cells through preconditioning is of great importance so that in vitro cell expansion may offer a valuable opportunity to achieve a large, sustainable cell population while simultaneously improving the NP cells' regenerative capacity 
$[11,12]$. In this study, we sought to determine whether variability existed in NP cells from herniated disc donors in response to independent preconditioning expansion strategies including hypoxic culture $(5 \%$ O2), decellularized ECM (DECM), fibroblast growth factor 2 (FGF2) supplementation, and plastic monolayer culture. Furthermore, we wanted to collect preliminary data for future determination of the correlation between the severity of disc degeneration and potential NP regeneration. 


\section{Materials and Methods}

\section{Preparation of ECM-Coated Flasks}

Plastic cell culture flasks were prepared as previously described [13]. Briefly, flasks were precoated with $0.2 \%$ gelatin (Sigma-Aldrich, St. Louis, MO) at $37^{\circ} \mathrm{C}$ for $1 \mathrm{~h}$ and seeded with passage 4 human adipose stem cells (Zen-Bio, Inc., Research Triangle Park, NC) at 6000 cells per cm2. After cells reached approximately $90 \%$ confluence, $250 \mu \mathrm{M}$ of L-ascorbic acid-2-phosphate (Wako Chemicals USA, Inc., Richmond, VA) was added for 10 days. The deposited ECM was incubated with $0.5 \%$ Triton $\mathrm{X}-100$ containing $20 \mathrm{mM}$ ammonium hydroxide at $37^{\circ} \mathrm{C}$ for 5 min to remove the cells; DECM was stored at $4^{\circ} \mathrm{C}$ in phosphate buffered saline (PBS) containing $100 \mathrm{U} / \mathrm{mL}$ penicillin, $100 \mu \mathrm{g} / \mathrm{mL}$ streptomycin, and $0.25 \mu \mathrm{g} / \mathrm{mL}$ fungizone until use.

\section{Cell Culture, Proliferation Analysis, and Immune Marker}

Human adult NP cells were obtained from the tissues of herniated disc patients (32-year-old male and 35-year-old female, no history of smoking, cardiac disease, or diabetes) following Institutional Review Board (IRB) approval and patient consent. Cells were cultured in growth medium containing Alpha Minimum Essential Medium ( $\alpha$ MEM), 10\% fetal bovine serum (FBS), 100 $\mathrm{U} / \mathrm{mL}$ penicillin, $100 \mu \mathrm{g} / \mathrm{mL}$ streptomycin, and $0.25 \mu \mathrm{g} / \mathrm{mL}$ fungizone. Passage $3 \mathrm{NP}$ cells from a grade II moderately degenerated (M-D) and grade IV severely degenerated (S-D) disc donors, respectively, were seeded onto four substrates with different conditions: plastic flasks (Control or “CNTL”) only; plastic flasks with 10 ng/mL of FGF2 (PeproTech Inc., Rocky Hill, NJ) (“FGF2”); DECM-coated cell culture flasks ("DECM"); and plastic flasks in a 5\% O2 cell culture incubator (hypoxia or "HYPO"). All groups except the HYPO group were cultured in 21\% O2 (normoxia) and $5 \% \mathrm{CO} 2$ in a $37^{\circ} \mathrm{C}$ incubator during the cell expansion phase. 
To determine proliferation index, before cell expansion, NP cells were labeled with CellVue ${ }^{\circledR}$ Claret at $2 \times 10-6 \mathrm{M}$ for $5 \mathrm{~min}$ according to the manufacturer's protocol (Sigma-Aldrich). After six days of proliferation, expanded cells were collected and measured using a BD FACS LSRFORTESSA $\square$ flow cytometer (BD Biosciences, San Jose, CA). Twenty thousand events of each sample were collected using CellQuest Pro software (BD Biosciences); cell proliferation index was analyzed using ModFit LT $\square$ version 3.1 (Verity Software House, Topsham, ME).

The following primary antibodies were used in flow cytometry analysis to detect expanded NP cell immunophenotype profiles: fluorescein isothiocyanate (FITC) conjugated CD95 (BioLegend, San Diego, CA), Phycoerythrin (PE) conjugated HLA-DR [major histocompatibility complex (MHC) class II] (BioLegend), and isotype-matched IgGs (Beckman Coulter, Fullerton, CA). Samples $(n=3)$ of each $2 \times 105$ expanded cells were incubated on ice in cold PBS containing $0.1 \%$ Chrom-Pure Human IgG whole molecule (Jackson ImmunoResearch Laboratories, West Grove, PA) and 1\% NaN3 (Sigma-Aldrich) for 30 min, followed by incubation in the dark in the primary antibody for $30 \mathrm{~min}$. Fluorescence signals were analyzed using a BD FACS LSRFORTESSA $\square$ flow cytometer and FCS Express 4 software package (De Novo Software, Los Angeles, CA).

\section{Redifferentiation Induction and Evaluation}

Expanded cells $(3 \times 105)$ were centrifuged at $500 \mathrm{~g}$ for $5 \mathrm{~min}$ in a $15-\mathrm{mL}$ polypropylene tube to form a pellet. After overnight incubation (day 0), the pellets were cultured in a serum-free chondrogenic medium consisting of high-glucose Dulbecco's Modified Eagle's Medium (DMEM), $40 \mu \mathrm{g} / \mathrm{mL}$ proline, $100 \mathrm{nM}$ dexamethasone, $100 \mathrm{U} / \mathrm{mL}$ penicillin, $100 \mu \mathrm{g} / \mathrm{mL}$ streptomycin, $0.1 \mathrm{mM}$ L-ascorbic acid-2-phosphate, and 1×ITS ${ }^{\mathrm{TM}}$ Premix (BD Biosciences) with the supplementation of $10 \mathrm{ng} / \mathrm{mL}$ transforming growth factor beta3 (TGF 33 , PeproTech, Inc.) in a 
$5 \% \mathrm{O} 2$ incubator as long as 21 days. The pellets were evaluated for NP cell redifferentiation using pellet size, biochemical analysis, and quantitative real-time polymerase chain reaction (qPCR).

Representative pellets ( $\mathrm{n}=4$ ) were digested at $60^{\circ} \mathrm{C}$ for $4 \mathrm{~h}$ with $125 \mu \mathrm{g} / \mathrm{mL}$ papain in PBE buffer (100 mM phosphate, $10 \mathrm{mM}$ ethylenediaminetetraacetic acid, $\mathrm{pH}$ 6.5) containing $10 \mathrm{mM}$ cysteine. To quantify cell density, the amount of DNA in the papain digestion was measured using the QuantiT $\square$ PicoGreen ${ }^{\circledR}$ dsDNA assay kit (Life Technologies, Carlsbad, CA) with a CytoFluor ${ }^{\circledR}$ Series 4000 (Applied Biosystems, Foster City, CA). GAG was measured using dimethylmethylene blue (DMMB) dye and a Spectronic $\square$ BioMate $\square 3$ Spectrophotometer (Thermo Fisher Scientific, Waltham, MA) with bovine chondroitin sulfate (Sigma-Aldrich) as a standard.

Total RNA was extracted from representative samples ( $n=4)$ using an RNase-free pestle in TRIzol® (Life Technologies). Two micrograms of mRNA were used for reverse transcriptase with High-Capacity cDNA Archive Kit (Applied Biosystems) at $37^{\circ} \mathrm{C}$ for $120 \mathrm{~min}$. NP cell redifferentiation related genes, type I collagen (COL1A1) (Assay ID Hs00164004_m1), aggrecan (ACAN) (Assay ID AIQJAP5), type II collagen (COL2A1) (Assay ID Hs00156568_m1), and type X collagen (COL10A1) (Assay ID Hs00166657), were customized by Applied Biosystems as part of their Custom TaqMan ${ }^{\circledR}$ Gene Expression Assays. Glyceraldehyde-3-Phosphate Dehydrogenase (GAPDH) (Assay ID Hs02758991_g1) was carried out as the endogenous control gene. TaqMan® real-time PCR was performed with the iCycler iQ $\square$ Multi Color Real-Time PCR Detection and calculated by computer software (Perkin-Elmer, Wellesley, MA). Relative transcript levels were calculated as $\chi=2-\Delta \Delta \mathrm{Ct}$, in which $\Delta \Delta \mathrm{Ct}=\Delta \mathrm{E}-\Delta \mathrm{C}, \Delta \mathrm{E}=\mathrm{Ctexp}-\mathrm{CtGAPDH}$, and $\Delta \mathrm{C}=\mathrm{Ctct} 1-$ CtGAPDH. 


\section{Statistical Analysis}

Analysis of variance (ANOVA) with pairwise comparison and t-test was used to compare measurements between different groups. All statistical analyses were performed with SPSS 13.0 statistical software (SPSS Inc., Chicago, IL). p values less than 0.05 were considered statistically significant. 


\section{Results}

NP Cells from Herniated Discs with Varied Degeneration Exhibited Dissimilar Characteristics

Spinal magnetic resonance imaging (MRI) of herniated disc patients prior to surgery and tissue collection revealed different degrees of disc degeneration (Fig. 1). M-D disc degeneration was deemed Grade II (moderately severe), while the S-D disc degeneration was deemed Grade IV (most severe). M-D disc and herniated NP tissue (L4-L5) appeared lighter in color with comparable density to the adjacent discs compared to S-D discs and maintained a reasonably distinct border between the annulus fibrosus (AF) and NP tissues. The S-D disc and herniated NP tissue (L5-S1) appeared to be a solid black color throughout indicating a loss of the AF and NP border and more degeneration. In culture, M-D disc cells divided and proliferated much quicker than S-D disc cells when expanded on plastic alone, with M-D disc cells exhibiting smaller, less flat, fibroblast-like morphology versus the flatter and larger S-D disc cells (not shown).

\section{DECM and FGF2 Preconditioning Elicited Immunophenotypic Changes}

HYPO preconditioning did not elicit a dramatic decrease in CD95 (Fas/APO-1) expression in either M-D or S-D disc cells versus control ( $2 \%$ reduction for each) (Fig. 2A/B/D). Interestingly, both FGF2 and DECM preconditioning significantly decreased the percentage of CD95(+) cells from $89.55 \%$ (control) to $67.63 \%$ with FGF2 treatment, to $69.21 \%$ with DECM expansion in M-

D disc cells; FGF2 and DECM preconditioning also significantly decreased the percentage of CD95(+) S-D disc cells from $67.98 \%$ (control) to $21.04 \%$ with FGF2 treatment, to $43.35 \%$ with DECM expansion (Fig. 2A/B/D). Both M-D and S-D disc cell populations were HLA-DR negative, even after DECM treatment (Fig. 2C). 


\section{DECM Preconditioning Enhanced M-D Proliferation and Redifferentiation}

The proliferation indices measured by flow cytometry were increased by HYPO and FGF2 preconditioning versus plastic CNTL in M-D disc cells, but was most pronounced in the DECM group (Fig. 3). Pellet morphology depicted the largest pellets in the DECM and FGF2 groups for M-D disc cell pellets (Fig. 4). In addition to cell proliferation and pellet sizes, DECM contributed to the greatest NP redifferentiation of expanded cells as evidenced by biochemistry and qPCR assays. M-D disc cells produced the greatest GAG content per pellet and greatest GAG/DNA ratio at day 10 and day 21 of redifferentiation (Fig. 5A/C). At day 21, the DECM group was the only group significantly increased versus plastic CNTL for GAG content per pellet ( $\mathrm{p}=0.0089)$, DNA ratio $(\mathrm{p}=0.0168)$, and GAG/DNA ( $\mathrm{p}=0.0223$ ) (Fig. 5A-C). Although HYPO preconditioning produced modest increases in proliferation, it failed to increase GAG content per pellet $(\mathrm{p}=0.3035)$, DNA ratio ( $\mathrm{p}=0.0802$ ), or GAG/DNA versus plastic CNTL ( $\mathrm{p}=0.9122$ ) (Fig. 5A-C). Both DECM and FGF2 produced the most significant decrease in COL1A1 $(\mathrm{p}=0.0003$ and $\mathrm{p}=0.0003$, respectively) and increase in ACAN mRNA expression ( $\mathrm{p}=0.0011$ and $\mathrm{p}=0.0241$, respectively). DECM preconditioning led to unmatched increases in COL2A1 mRNA expression, at 3.5 -fold greater than plastic CNTL ( $\mathrm{p}=0.0021)$ (Fig. 6A-C).

Preconditioning Strategies Failed to Rejuvenate S-D NP Cells

Although FGF2 and DECM, but not HYPO preconditioning, were able to increase cell proliferation versus plastic CNTL (Fig. 2), the S-D disc cells did not increase their redifferentiation capacity with any preconditioning treatments. At day 10 of redifferentiation, FGF2 and DECM treatments in S-D disc cells both produced the smallest pellets of any group (Fig. 4). Additionally, at Day 21, FGF2 pellets seemed to decrease further in size (Fig. 4), and was accompanied by a 
decrease in DNA ratio ( $\mathrm{p}=0.0000$ ) (Fig. 5E), indicative of a decrease in cell viability; GAG content per pellet $(\mathrm{p}=0.0000)$ and GAG/DNA $(\mathrm{p}=0.0000)$ also decreased, indicative of a decrease in deposited matrix, versus plastic CNTL (Fig. 5D/F). This finding is dissimilar to the results obtained from M-D disc cells. None of the preconditioning treatments significantly increased GAG content per pellet or GAG/DNA versus plastic CNTL (Fig. 5D/F). DNA ratio was only increased in the HYPO ( $\mathrm{p}=0.0224)$ and DECM groups $(\mathrm{p}=0.0022)$ at Day 21 (Fig. 5E). Despite preconditioning interventions, COL1A1 mRNA was significantly increased in all groups versus plastic CNTL $(\mathrm{p}=0.0055, \mathrm{p}=0.0002$, and $\mathrm{p}=0.0174$, respectively $)$ (Fig. 6E) and ACAN mRNA was decreased in the DECM and FGF2 groups ( $\mathrm{p}=0.0377$ and $\mathrm{p}=0.0010$, respectively) (Fig. 6F). Despite these failures in increased redifferentiation capacity, COL2A1 expression was significantly increased in the HYPO and DECM groups versus plastic CNTL ( $\mathrm{p}=0.0259$ and $\mathrm{p}=0.0048$, respectively) (Fig. $6 G)$. 


\section{Discussion}

In the current study, we wanted to determine whether there were any discrepancies in the responses of NP cells harvested from donors with varying degrees of disc degeneration to FGF2, DECM, or HYPO preconditioning during expansion and redifferentiation in vitro. We found that preconditioning strategies unequally impacted regeneration of NP cells from human herniated discs: cells harvested from a moderately degenerated NP donor responded well to DECM and FGF2 treatments, but cells from a severely degenerated donor were not successfully rejuvenated and redifferentiated. The present study suggests that specific expansion methods may be more appropriate for one patient versus another, supporting the call for attention to personalized medical interventions and cell therapies based upon initial medical diagnoses and assessments of NP degeneration. Additionally, it may be necessary for alternative therapies to be developed for the expansion and rejuvenation of severely degenerated NP tissues.

In order to provide preliminary data for determining the correlation between disc degeneration clinical data and isolated disc cell biological performance, two representative disc degeneration models, Grade II (moderately severe) and Grade IV (most severe), were used and defined based on well-established T2-MRI grading classifications [14,15] with the assistance of orthopaedic spine surgeons. A previous report suggested that young and healthy DECM is a promising cell expansion system for stem cell rejuvenation [16]; however, an allogeneic source of DECM from young and healthy donors, an ideal and commercializable approach, might cause immune concerns for the expanded cells after transplantation. This concern may diminish due to this study's finding that both moderately and severely degenerated NP cells remained HLA-DR negative after pretreatment with allogeneic DECM, in line with the results from another human degenerated disc study [17] and human synovium-derived stem cell (SDSC) study [18]. 
Previous studies have implicated CD95 (Fas receptor) in degenerated and herniated human discs [19-21], in disc immune privilege [22,23], and as an important regulator of caspase-mediated cell death via apoptosis [24,25]. Furthermore, it has been reported that CD95 expression is greater in NP from non-contained versus contained disc herniations and, after herniation occurs, disc cells may undergo Fas-mediated apoptosis [20]. In the current study, both FGF2 and DECM preconditioning resulted in a decrease in the percentage of $\mathrm{CD} 95(+)$ cells in both moderately and severely degenerated disc cells, but not with HYPO preconditioning. Given the known role of CD95 in Fas-mediated apoptosis, this finding suggests that certain preconditioning methods, particularly DECM and FGF2 treatments, could potentially rejuvenate NP cells to a younger, less immunogenic phenotype.

Our results indicated that, compared with severely degenerated disc cells, cell viability after 21 days of redifferentiation induction was not affected by HYPO preconditioning in moderately degenerated disc cells, as evidenced by no significant differences in the DNA ratio versus control. We also found that both moderately and severely degenerated disc cells were not successfully induced for redifferentiation by HYPO preconditioning except for an increase of the COL2A1 mRNA in the pellets from severely degenerated disc cells after 21-day induction. Our finding is in line with a recent study investigating the hypoxic culture of human NP cells; Yang and colleagues found that NP cells expanded under $21 \%$ oxygen and then later cultured in hypoxia ( $3.5 \%$ oxygen) could only be partially "rescued" to increase COL2A1 and ACAN expression versus cells initially cultured under $3.5 \%$ oxygen exclusively [26]. Despite minimal benefits from HYPO preconditioning alone, the combination with FGF2 and DECM exhibited the largest contribution in the rejuvenation of porcine SDSCs for both proliferation and chondrogenic potential [27]. Since hypoxia-induced factor 1 alpha (HIF1 $\alpha$ ) has been implicated in NP cell survival and ECM 
homeostasis in HIF1 $\alpha$ knockout mouse models [28], the elucidation of potential mechanisms underlying HYPO preconditioning becomes increasingly important in utilizing this strategy for cell-based disc regeneration.

In this study, FGF2 treatment of $10 \mathrm{ng} / \mathrm{mL}$ during NP cell expansion modestly increased the proliferation indices, which was expected since FGF2 is a well-known mitogen and has been utilized in the expansion of NP cells in other studies [8]. This function of FGF2 might be explained by its anti-anabolic effects through the upregulation of NOGGIN via the mitogen-activated protein kinase (MAPK) and Nuclear Factor-KappaB (NF-кB) pathways [29]. Furthermore, we found that supplementation of FGF2 during expansion enhanced the redifferentiation capacity of moderately degenerated disc cells but not severely degenerated disc cells. Our results were partially supported by a previous investigation, in which FGF2 preconditioning had no effect on cell proliferation, but could restore COL2A1, SOX9 [SRY (Sex Determining Region Y)-Box 9], COMP (cartilage oligomeric matrix protein), CHAD (chondroadherin), and FMOD (fibromodulin) as well as maintain Smad2/3 mediated TGF $\beta$ signaling in expanded NP cells during subsequent incubation in alginate [30]. Another group found that the addition of $2 \mathrm{ng} / \mathrm{mL}$ of FGF2 during expansion resulted in a slight increase in proliferation of cervical disc cells harvested from both mildly and severely degenerated discs but FGF2 appeared to be insignificant in the redifferentiation process [17].

Overall, NP cells from both donors benefited by DECM expansion versus other pretreatments in terms of proliferation and redifferentiation. These effects were more pronounced in the cells from the moderately degenerated donor than from the severely degenerated donor. This finding is consistent with previous reports, where NP cells expanded on an SDSC DECM increased proliferative and redifferentiation potential [31,32]. Interestingly, a recent study found that 
increasing GAG synthesis and combatting degradation was an effective strategy in mildly degenerated, but not severely degenerated disc cells [33]. Although many studies have reported successful increases in NP redifferentiation with the use of biomaterial scaffolds and hydrogels, extensively reviewed by Priyadarshani and colleagues [34], more comparative research studies are needed, especially those investigating the differences between mildly and severely degenerated disc cells and their ability to undergo redifferentiation following 3D-expansion strategies.

Disease pathology and donor specific responses should be considered when choosing appropriate cell expansion strategies for cell-based interventions. To our knowledge, this study is the first to evaluate and report preconditioning strategies using FGF2 and DECM expansion to decrease CD95 (Fas) expression in degenerated NP cells; additionally, this study provides direct comparison of several preconditioning strategies in a single controlled study. This study supports the need for personalized medicine based on disease pathology and assessment, as evidenced by the donordependent responses to identical preconditioning stimuli. Based on the results from the current study, it seems that DECM and FGF2 could be beneficial treatments for NP cells obtained from patients with mildly to moderately degenerative intervertebral discs. Further investigation of disc degeneration and preconditioning responses is necessary to validate this hypothesis.

For cell-based treatment interventions in both moderate and severe disc degeneration, clinicians should consider the degree of disc degeneration in choosing the most appropriate preconditioning strategy. Although this proof of principle study presents new data regarding preconditioning strategies for increasing disc cell proliferation and redifferentiation and decreasing CD95 expression, there were recognized limitations in this study, which include the influence of the number of donor tissues, age, gender, herniated type, and disease duration. These limitations should be considered when designing future studies to investigate the correlation between the 
severity of disc degeneration and potential NP regeneration. Ultimately, our goal is to be able to predict the regenerative potential of harvested disc cells at a radiological level due to its inherent relationship with intervertebral disc health. In the future, defining the correlation between clinical MRI data and the potential biological activity of patient NP cells could serve as a significant breakthrough from improving cell-based disc therapies. 


\section{Disclosure Statement}

No competing financial interests exist. 


\section{References}

1. Hoy D, Bain C, Williams G, March L, Brooks P, Blyth F, et al. A systematic review of the global prevalence of low back pain. Arthritis Rheum 2012;64:2028-37.

2. Walsh NE, Brooks P, Hazes JM, Walsh RM, Dreinhöfer K, Woolf AD, et al. Bone and Joint Decade Task Force for Standards of Care for Acute and Chronic Musculoskeletal Pain. Standards of care for acute and chronic musculoskeletal pain: the Bone and Joint Decade (20002010). Arch Phys Med Rehabil 2008;89:1830-45.

3. Hayes AJ, Hughes CE, Ralphs JR, Caterson B. Chondroitin sulphate sulphation motif expression in the ontogeny of the intervertebral disc. Eur Cell Mater 2011;7:1-14.

4. Sivan SS, Hayes AJ, Wachtel E, Caterson B, Merkher Y, Maroudas A, et al. Biochemical composition and turnover of the extracellular matrix of the normal and degenerate intervertebral disc. Eur Spine J 2014;23S3:S344-53.

5. Huang YC, Leung VY, Lu WW, Luk KD. The effects of microenvironment in mesenchymal stem cell-based regeneration of intervertebral disc. Spine J 2013;13:352-62.

6. Ganey T, Libera J, Moos V, Alasevic O, Fritsch KG, Meisel HJ, et al. Disc chondrocyte transplantation in a canine model: a treatment for degenerated or damaged intervertebral disc. Spine (Phila Pa 1976) 2003;28:2609-20.

7. Hohaus C, Ganey TM, Minkus Y, Meisel HJ. Cell transplantation in lumbar spine disc degeneration disease. Eur Spine J 2008;17 Suppl 4:492-503.

8. Hegewald AA, Endres M, Abbushi A, Cabraja M, Woiciechowsky C, Schmieder K, et al. Adequacy of herniated disc tissue as a cell source for nucleus pulposus regeneration. J Neurosurg Spine 2011;14:273-80.

9. Li JT, Pei M. Cell senescence: a challenge in cartilage engineering and regeneration. Tissue Eng Part B 2012;18:270-87.

10. Zhang Y, Pizzute T, Pei M. Anti-inflammatory strategies in cartilage repair. Tissue Eng Part B 2014;20:655-68.

11. Pei M. Environmental preconditioning rejuvenates adult stem cells' proliferation and chondrogenic potential. Biomaterials 2016; doi: 10.1016/j.biomaterials.2016.11.049. 
12. Shoukry M, Li J, Pei M. Reconstruction of an in vitro niche for the transition from intervertebral disc development to nucleus pulposus regeneration. Stem Cells Dev 2013;22:116276.

13. He F, Chen X, Pei M. Reconstruction of an in vitro tissue-specific microenvironment to rejuvenate synovium-derived stem cells for cartilage tissue engineering. Tissue Eng Part A 2009;15:3809-21.

14. Jacobs LJ, Chen AF, Kang JD, Lee JY. Reliable Magnetic Resonance Imaging Based Grading System for Cervical Intervertebral Disc Degeneration. Asian Spine J 2016;10:70-4.

15. Watanabe A, Benneker LM, Boesch C, Watanabe T, Obata T, Anderson SE. Classification of intervertebral disk degeneration with axial T2 mapping. AJR Am J Roentgenol 2007;189:93642.

16. Li J, Hansen KC, Zhang Y, Dong C, Dinu CZ, Dzieciatkowska M, et al. Rejuvenation of chondrogenic potential in a young stem cell microenvironment. Biomaterials 2014;35:642-53.

17. Stich S, Stolk M, Girod PP, Thomé C, Sittinger M, Ringe J, et al. Regenerative and immunogenic characteristics of cultured nucleus pulposus cells from human cervical intervertebral discs. PLoS One 2015;10:e0126954.

18. Zhang Y, Pizzute T, Li J, He F, Pei M. sb203580 preconditioning recharges matrixexpanded human adult stem cells for chondrogenesis in an inflammatory environment - A feasible approach for autologous stem cell based osteoarthritic cartilage repair. Biomaterials 2015;64:8897.

19. Han D, Ding Y, Liu SL, Wang G, Si IC, Wang X, et al. Double role of Fas ligand in the apoptosis of intervertebral disc cells in vitro. Acta Biochim Biophys Sin (Shanghai) 2009;4:93847.

20. Park JB, Kim KW, Han CW, Chang H. Expression of Fas receptor on disc cells in herniated lumbar disc tissue. Spine (Phila Pa 1976) 2001;26:142-6.

21. Sun Z, Wan ZY, Liu ZH, Guo YS, Yin JB, Duan CG, et al. Expression of soluble Fas and soluble FasL in human nucleus pulposus cells. Int J Clin Exp Pathol 2013;6:1567-73.

22. Sun Z, Liu ZH, Chen YF, Zhang YZ, Wan ZY, Zhang WL, Che L, Liu X, Wang HQ, Luo ZJ. Molecular immunotherapy might shed a light on the treatment strategies for disc degeneration and herniation. Med Hypotheses 2013;81:477-80. 
23. Wang J, Tang T, Yang H, Yao X, Chen L, Liu W, et al. The expression of Fas ligand on normal and stabbed-disc cells in a rabbit model of intervertebral disc degeneration: a possible pathogenesis. J Neurosurg Spine 2007;6:425-30.

24. Fu Q, Fu TM, Cruz AC, Sengupta P, Thomas SK, Wang S, et al. Structural Basis and Functional Role of Intramembrane Trimerization of the Fas/CD95 Death Receptor. Mol Cell 2016;61:602-13.

25. Imai Y, Kimura T, Murakami A, Yajima N, Sakamaki K, Yonehara S. The CED-4homologous protein FLASH is involved in Fas-mediated activation of caspase-8 during apoptosis. Nature 1999;398:777-85.

26. Yang SH, Hu MH, Sun YH, Lin FH. Differential phenotypic behaviors of human degenerative nucleus pulposus cells under normoxic and hypoxic conditions: influence of oxygen concentration during isolation, expansion, and cultivation. Spine J 2013;13:1590-6.

27. Li J, Pei M. Optimization of an in vitro three-dimensional microenvironment to reprogram synovium-derived stem cells for cartilage tissue engineering. Tissue Eng Part A 2011;17:703-12.

28. Wu WJ, Zhang XK, Zheng XF, Yang YH, Jiang SD, Jiang LS. SHH-dependent knockout of HIF-1 alpha accelerates the degenerative process in mouse intervertebral disc. Int J Immunopathol Pharmacol 2013;26:601-9.

29. Li X, An HS, Ellman M, Phillips F, Thonar EJ, Park DK, et al. Action of fibroblast growth factor-2 on the intervertebral disc. Arthritis Res Ther 2008;10:R48.

30. Tsai TT, Guttapalli A, Oguz E, Chen LH, Vaccaro AR, Albert TJ, et al. Fibroblast growth factor-2 maintains the differentiation potential of nucleus pulposus cells in vitro: implications for cell-based transplantation therapy. Spine (Phila Pa 1976) 2007;32:495-502.

31. He F, Pei M. Rejuvenation of nucleus pulposus cells using extracellular matrix deposited by synovium-derived stem cells. Spine (Phila Pa 1976) 2012;37:459-69.

32. Pei M, Shoukry M, Li J, Daffner SD, France JC, Emery SE. Modulation of in vitro microenvironment facilitates synovium-derived stem cell-based nucleus pulposus tissue regeneration. Spine (Phila Pa 1976) 2012;37:1538-47

33. Zhu Q, Gao X, Temple HT, Brown MD, Gu W. Simulation of biological therapies for degenerated intervertebral discs. J Orthop Res 2016;34:699-708.

34. Priyadarshani P, Li Y, Yao L. Advances in biological therapy for nucleus pulposus regeneration. Osteoarthritis Cartilage 2016;24:206-12. 
Figure 4.1:

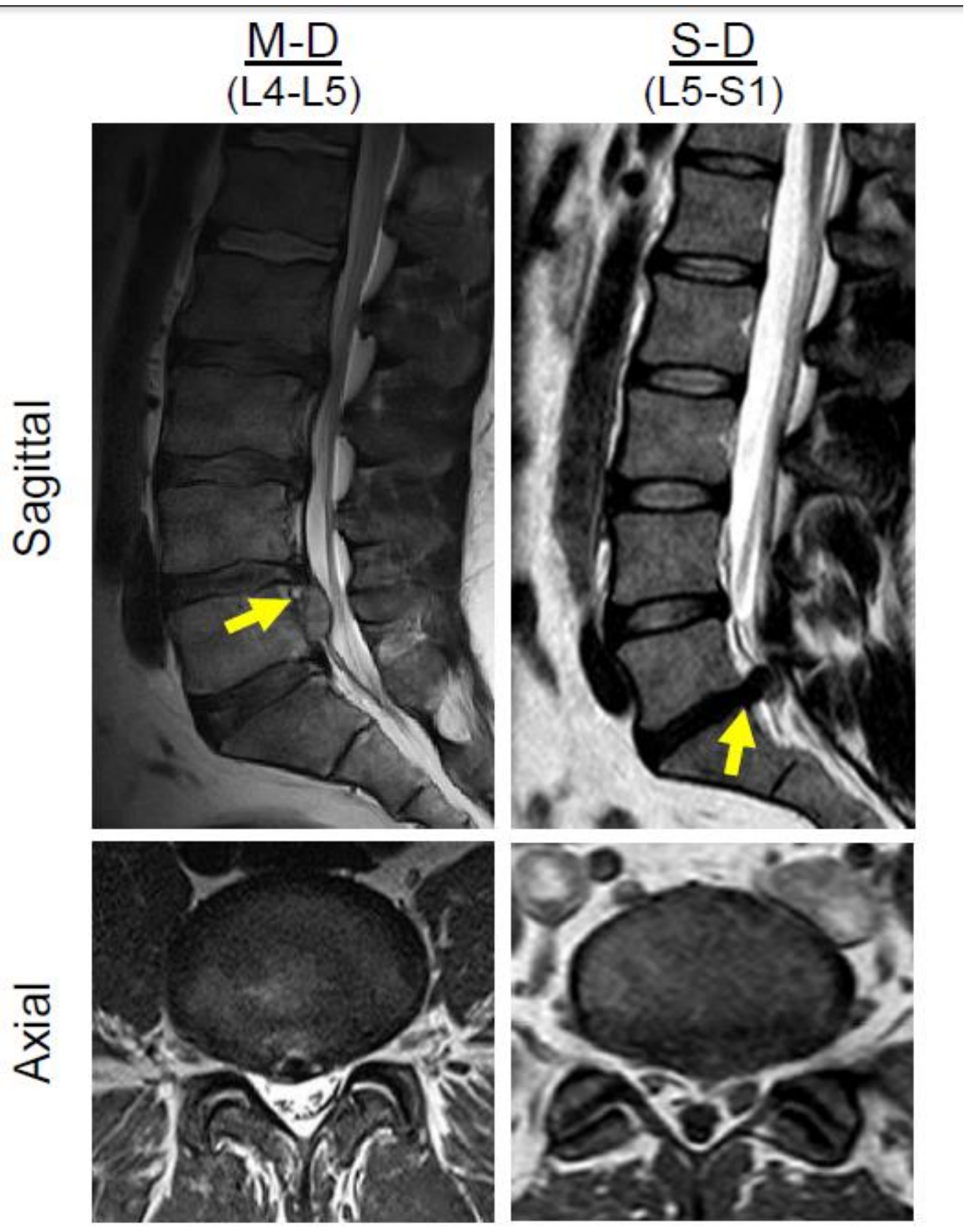


Figure 4.1: Patient MRIs. MRI T2 images from herniated disc patients (M-D and S-D) who served as representative NP cell donors in this study. The arrow points to the herniated discs. 
Figure 4.2:

A

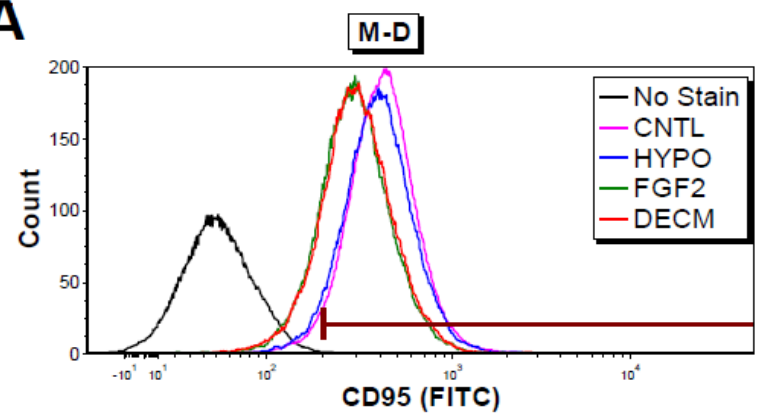

B

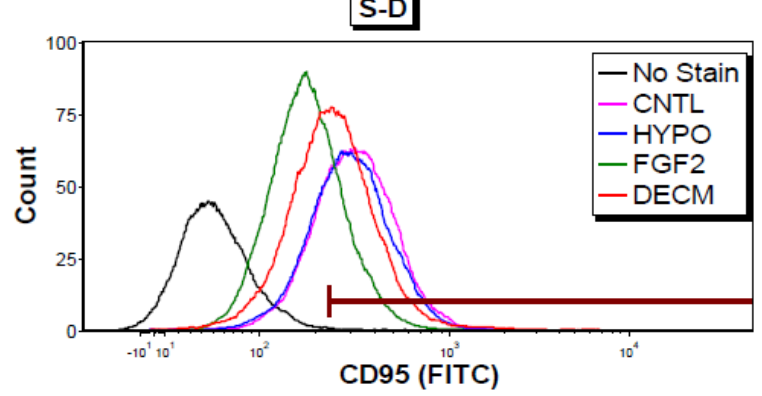

C

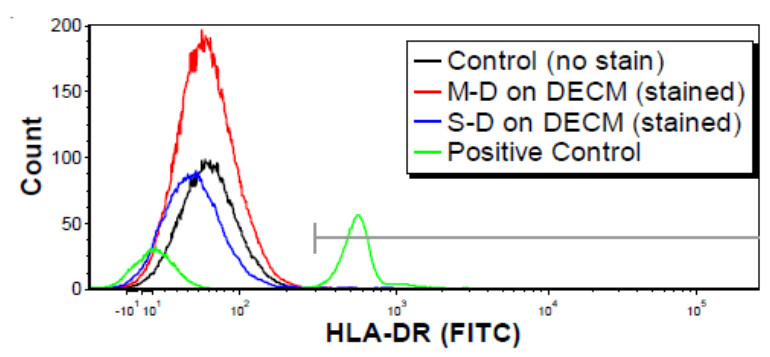

D

\begin{tabular}{|c|c|c|}
\hline Group & CD95(+) M-D & CD95(+) S-D \\
\hline CNTL & $89.55 \%$ & $67.98 \%$ \\
\hline HYPO & $87.87 \%$ & $65.10 \%$ \\
\hline FGF2 & $67.63 \%$ & $21.04 \%$ \\
\hline DECM & $69.21 \%$ & $43.35 \%$ \\
\hline
\end{tabular}


Figure 4.2: CD95 and HLA-DR expression of preconditioned NP cells. Following cell expansion under varied preconditioning approaches using hypoxia (HYPO), FGF2, and DECM with the plastic flask group as a control (CNTL), NP cells from two different severities of degenerated donor discs (M-D and S-D) were evaluated for CD95 (A-B) and HLA-DR (C) expression via flow cytometry, as well as percentages of CD95(+) NP cells for each treatment and donor (D). 
Figure 4.3:
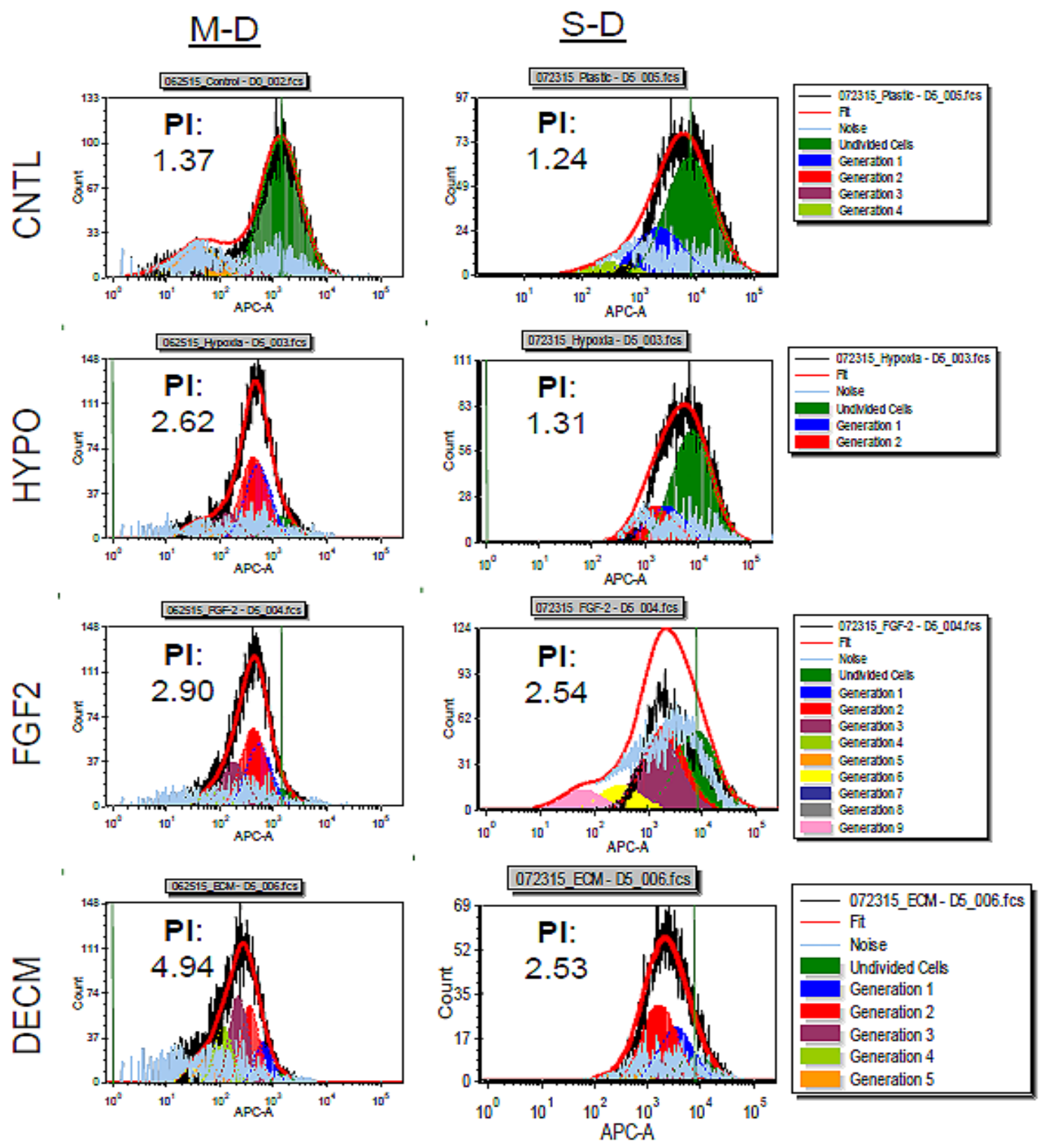
Figure 4.3: Proliferation analysis of expanded NP cells. Following cell expansion and preconditioning, pre-stained NP cells from the donors (M-D and S-D) were evaluated for proliferation index by flow cytometry. 
Figure 4.4:

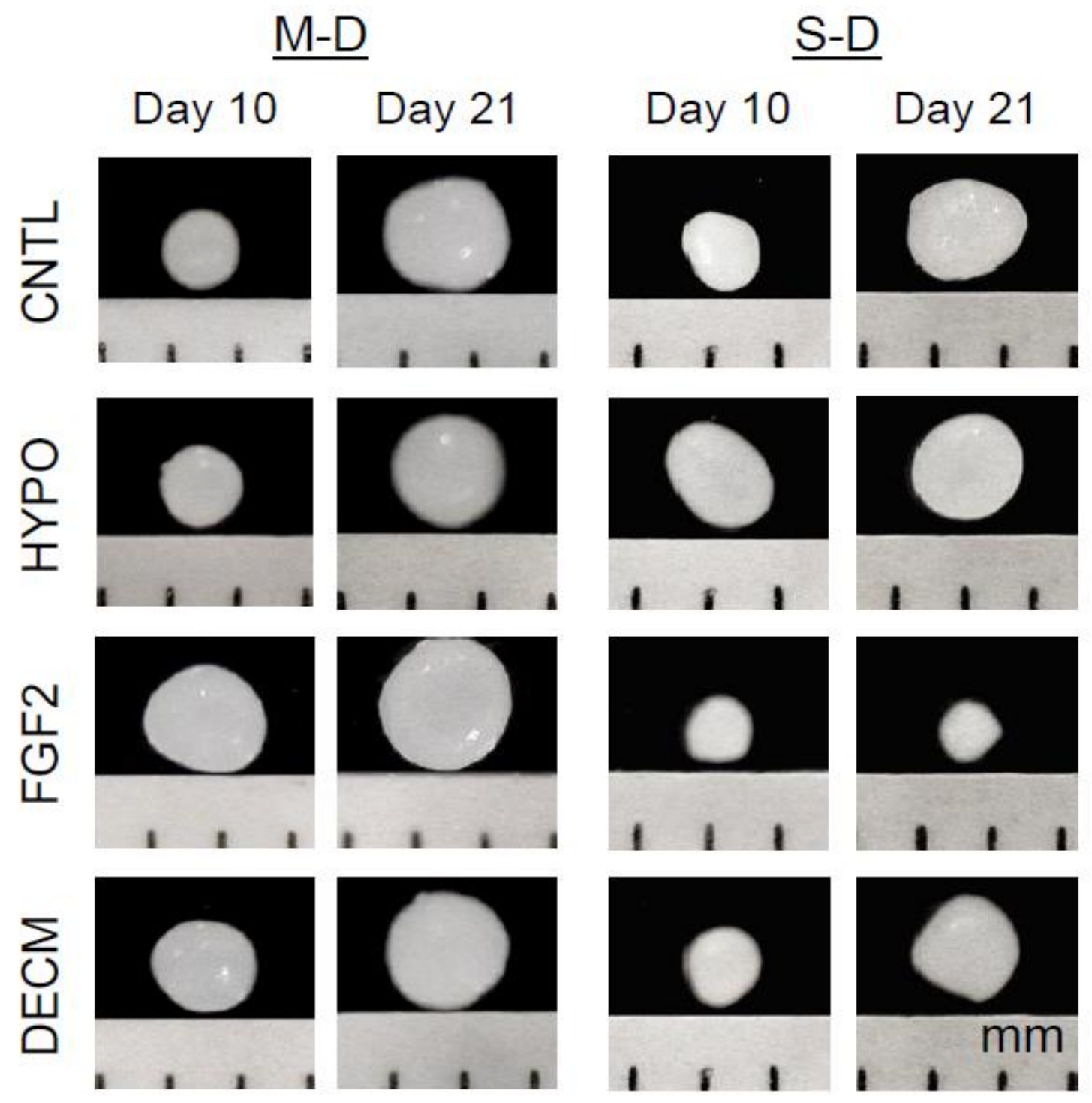


Figure 4.4: NP cell pellet morphology and size following preconditioning expansion and redifferentiation induction. After preconditioning under HYPO, FGF2, and DECM, with the plastic flask group as a control (CNTL), cell pellets were subjected to redifferentiation media and harvested for evaluation at day 10 and day 21 from each preconditioning group. 
Figure 4.5:

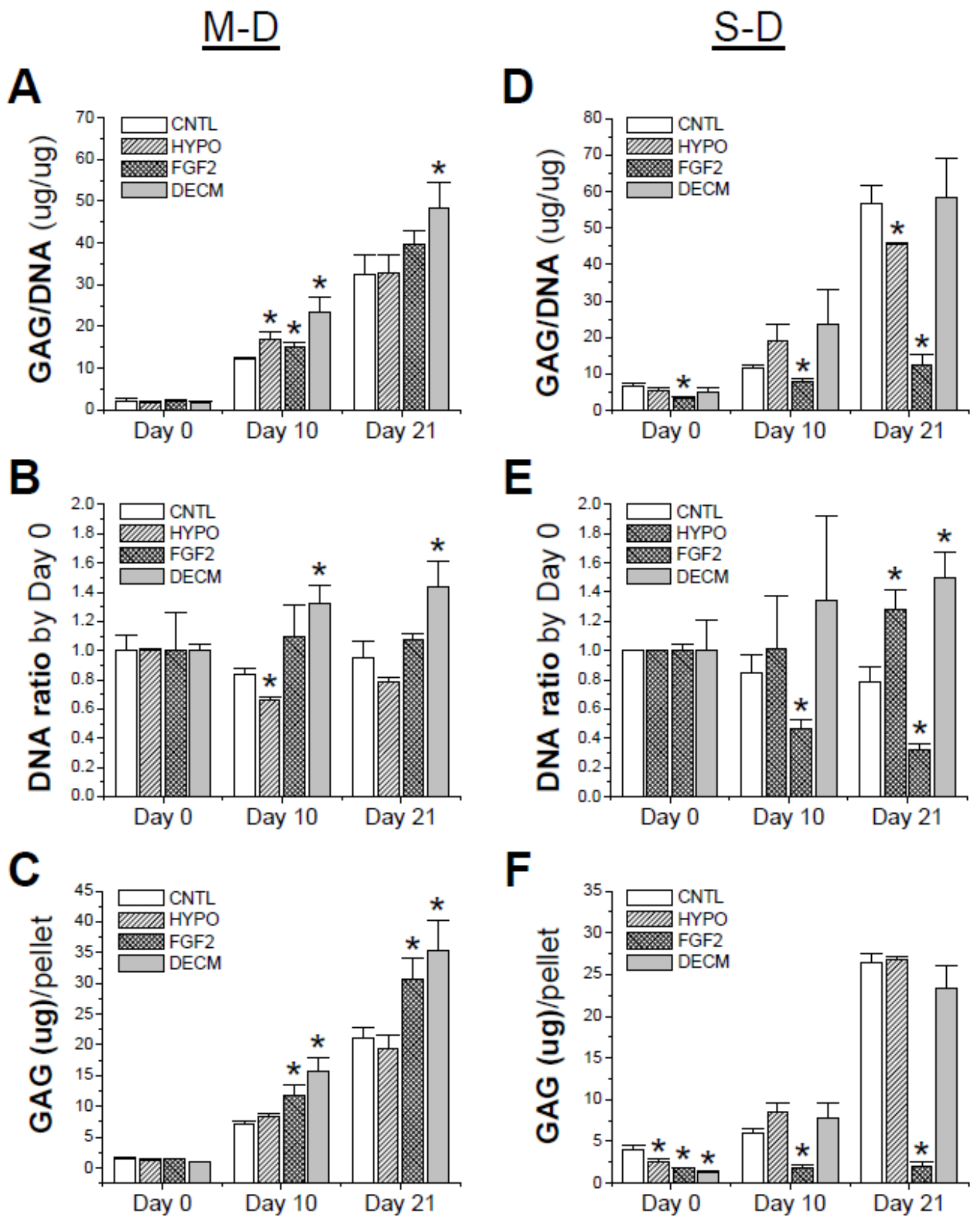


Fig. 4.5. Biochemical analysis of redifferentiated pellets. Glycosaminoglycan (GAG) and DNA contents were evaluated for M-D (A-C) and S-D (D-F) donors at day 0, 10, and 21 of pellet redifferentiation from each preconditioning group. Data are shown as average \pm SD for $n=4$. * indicates a statistical difference compared with the corresponding CNTL group $(p<0.05)$. 
Figure 4.6:

\section{M-D}

A

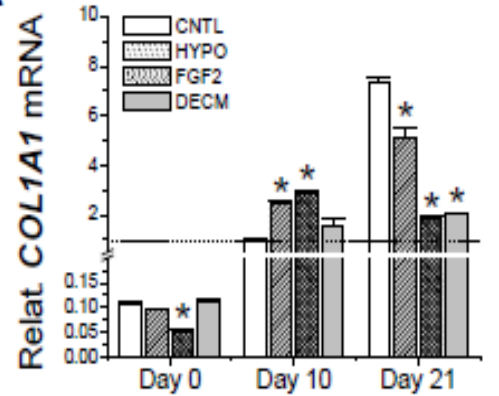

B

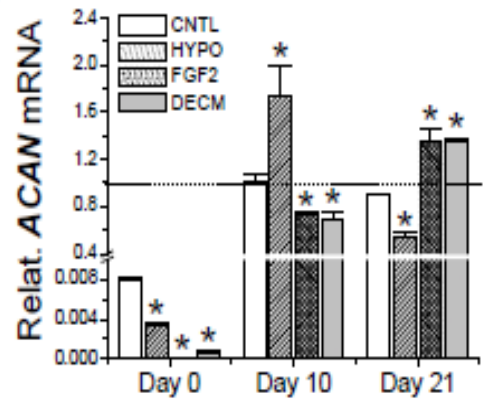

C

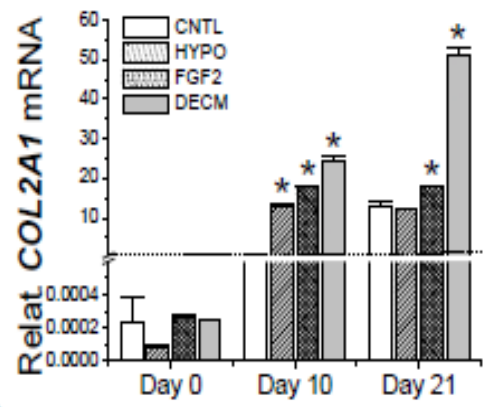

D

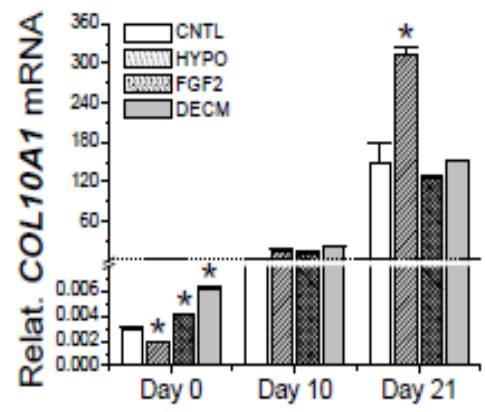

S-D

E

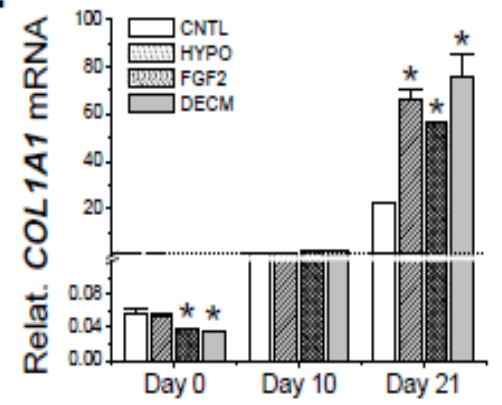

F

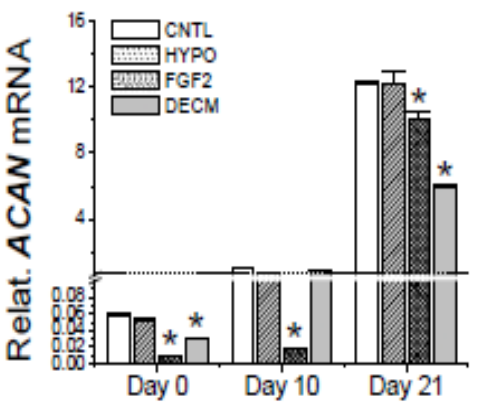

G

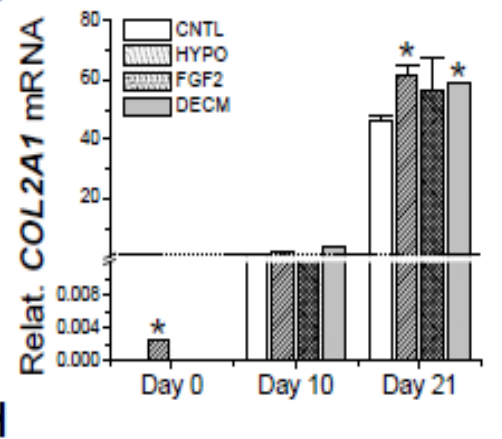

$\mathrm{H}$

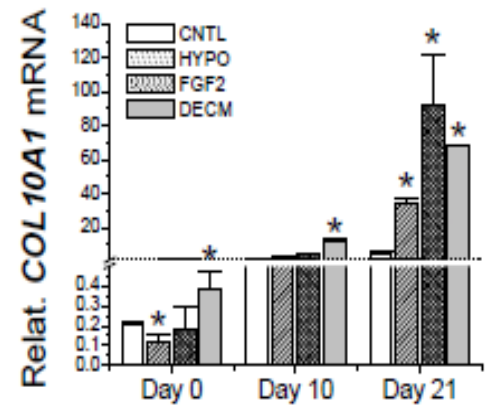


Figure 4.6: TaqMan ${ }^{\circledR}$ real-time PCR of redifferentiation pellets. M-D (A-D) and S-D (E-H) donor cell pellets were evaluated for COL1A1, ACAN, COL2A1, and COL1OA1 mRNA expression for each preconditioning group. Data are shown as average \pm SD for $n=4$. * indicates a statistical difference compared with the corresponding CNTL group $(p<0.05)$. 


\section{Chapter 5:}

\section{Extracellular matrix preconditioning of nucleus pulposus cells and impacts on immunity}

In preparation for submission; TBD 2017

Tyler T. Pizzute ${ }^{1}$, Ming Pei ${ }^{1}$.

${ }^{1}$ Stem Cell and Tissue Engineering Laboratory, Department of Orthopaedics, West Virginia University, One Medical Center Drive, PO Box 9196, Morgantown, WV 26506-9196, USA.

Running Title: Matrix and NP immunity

Corresponding author: Ming Pei, $\mathrm{MD}, \mathrm{PhD}$

Stem Cell and Tissue Engineering Laboratory, Department of Orthopaedics, West Virginia University, PO Box 9196, One Medical Center Drive, Morgantown, WV 26506-9196, USA, Telephone: 304-293-1072; Fax: 304-293-7070; Email: mpei@hsc.wvu.edu 


\section{Abstract}

Preconditioning of human cells and the use of three-dimensional (3D), biologically-derived substrates to expand them has gained attention in recent years, with the goal of safely increasing cell yield to obtain a healthy cell population. This goal is especially important in the preconditioning of nucleus pulposus cells (NPCs) of the intervertebral disc (IVD), because their use in cell-based regenerative therapies is generally limited by cell number and poor regenerative capacity upon harvest. In addition to these potential benefits, it is vital that the preconditioning method does not compromise the stemness or increase susceptibility to adverse immune reactions. These issues pose serious concerns for preconditioning methods that utilize biologically-derived components for cell expansion such as decellularized extracellular matrix (dECM), or other methods that allow for allogeneic and xenogeneic exposures. In this study, we compared the immunophenotypes of human NPCs, harvested from lumbar IVDs and expanded on plastic (Plastic), autologous dECM (AutoECM), allogeneic dECM (AlloECM), and xenogeneic dECM (XenoECM) substrates; surface markers assayed included HLA-DR, CD80, CD86, and CD95 (Fas). After expansion, cells were co-cultured with allogeneic peripheral blood mononuclear cells (PBMCs) in mixed lymphocyte reactions and assayed for T-cell proliferation. Xenogeneic and allogeneic dECMs were also assayed for redifferentiation following cell expansion. Overall, this study presents new data suggesting that dECM expansion, irrespective of source (autologous, allogeneic, or xenogeneic cell-based) allowed human herniated NPCs to express similar immunophenotypes and immunological responses for T-cell proliferation compared to plastic expansion alone. These results suggest that dECMs may be an effective, immunologically acceptable method for NPC preconditioning and cell expansion. 


\section{Introduction}

Healthcare costs and annual spending for back pain related visits are on the rise [1]; a major contributor to these increases are attributed to spinal injury and related pain [2], as well as disc degeneration and herniation [3]. Therefore, therapies that can provide treatment of ailing discs are gaining recent attention. Recently, cell-mediated therapies have gained attention for repair or treatment of intervertebral discs (IVDs) [4] [5], with the goals of increasing disc cellularity, lost nucleus pulposus (NP) tissue and disc height [6] [7]. Although many preconditioning strategies can be employed [8], 3-dimensional (3D) cultures have been a recent area of interest for the development of clinical solutions. One preconditioning and expansion method is the use of decellularized extracellular matrix (dECM) expansion of harvested patient cells, which has been demonstrated as a successful cell expansion method to increase yield and rejuvenation of stem cell and nucleus pulposus cells alike [9] [10] [11]. Despite the success of 3D cultures in terms of cell differentiation potential [11] [12] [13], few studies have focused on potential deleterious effects on the immunogenic properties of expanded cells. It has been suggested that biomaterial-induced alterations in immunological responses, particularly those resulting from cell-based approaches, are important and frequently overlooked factors that can contribute to immunogenicity [13]. Because the inner space of the IVD is an inherently immune-privileged site [14], immunogenicity is an area of concern when new cells are introduced [15] [16].

Due to the known roles of major histocompatibility complex-II (MHC-II or HLA-DR) and its role in immune recognition and graft rejection [17] [18] [19], we hypothesize that HLA-DR, as well as co-stimulator markers CD80/CD86 [20] may be involved in immune issues related to preconditioning and cell expansion. Recent studies support this hypothesis; Yuan and colleagues, who have previously reported that seeding cells in collagen hydrogels and their introduction in 
vivo increased MHC-II (HLA-DR) expression and suggested that fast matrix formation and mindful scaffold selection may be useful strategies [21]. Similarly, Zhang and colleagues have reported increases in HLA-DR expression in synovial stem cells with xenogeneic dECM expansion [22] and conversely, Pizzute and colleagues reported that allogeneic adipose-derived stem cell dECM did not elicit this same increase [23].

Human NPCs have been shown to express Fas and Fas ligand [24] [25], and serve as a method for maintaining immune privilege in the disc through apoptotic interactions with invading immune cells [26] [27]. Fas receptor (CD95) is a surface receptor involved in apoptosis [28] [29], and when bound by Fas ligand (CD178), leads to downstream activation of caspase-8 [28] [30] and, ultimately, apoptosis. Fas has been specifically implicated in degenerative IVD cells [24] [31] and despite its role in immune privilege of the IVD, Fas has been further implicated as a disadvantageous surface marker for NPCs, primarily due to the potential for cytotoxic T-cell activity and cell-mediated apoptosis of disc cells [13]. Overall, in this study, we aimed to provide new insight into the potential immune issues surrounding NPC expansion on 3D, biologicallyderived substrates such as allogeneic and xenogeneic dECMs. 


\section{Materials and Methods}

Preparation of ECM-coated flasks

Plastic cell culture flasks were prepared as previously described [11]. Briefly, flasks were precoated with $0.2 \%$ gelatin (Sigma-Aldrich, St. Louis, MO) at $37^{\circ} \mathrm{C}$ for $1 \mathrm{~h}$ and seeded with passage 2 human or porcine nucleus pulposus cells at 6000 cells per $\mathrm{cm}^{2}$. After cells reached approximately 90\% confluence, $250 \mu \mathrm{M}$ of L-ascorbic acid-2-phosphate (Wako Chemicals USA, Inc., Richmond, VA) was added for 10 days. The deposited ECM was incubated with $0.5 \%$ Triton X-100 containing $20 \mathrm{mM}$ ammonium hydroxide at $37^{\circ} \mathrm{C}$ for $5 \mathrm{~min}$ to remove the cells; DECM was stored at $4{ }^{\circ} \mathrm{C}$ in phosphate buffered saline (PBS) containing $100 \mathrm{U} / \mathrm{mL}$ penicillin, $100 \mu \mathrm{g} / \mathrm{mL}$ streptomycin, and $0.25 \mu \mathrm{g} / \mathrm{mL}$ fungizone until use.

\section{Cell culture}

Human adult NP cells were previously obtained from the tissues of herniated disc patients (32year-old male and 35-year-old female) following patient consent and Institutional Review Board (IRB) approval. Cells were cultured in growth medium containing Alpha Minimum Essential Medium ( $\alpha \mathrm{MEM}), 10 \%$ fetal bovine serum (FBS), $100 \mathrm{U} / \mathrm{mL}$ penicillin, $100 \mu \mathrm{g} / \mathrm{mL}$ streptomycin, and $0.25 \mu \mathrm{g} / \mathrm{mL}$ fungizone. After reaching confluence, cells were detached with $1 \mathrm{X}$ TrypsinEDTA and seeded on plastic (Plastic) flasks as a control, or on previously prepared dECM flasks from autologous (AutoECM), allogeneic (AlloECM), or porcine-derived xenogeneic (XenoECM) NPC sources under the same culture media conditions, changing media every third day. 


\section{Flow cytometry and cell proliferation analyses}

To determine the proliferation index of each group (Fig 1), before cell expansion, NP cells were pre-labeled with CellVue ${ }^{\circledR}$ Claret at $2 \times 10^{-6} \mathrm{M}$ for 5 min according to the manufacturer's protocol (Sigma-Aldrich). After six days of proliferation, expanded cells were collected and measured using a BD FACS LSRFORTESSA ${ }^{\text {TM }}$ flow cytometer (BD Biosciences, San Jose, CA). Twenty thousand events of each sample were collected using CellQuest Pro software (BD Biosciences); cell proliferation index was analyzed using ModFit LT $^{\mathrm{TM}}$ version 3.1 (Verity Software House, Topsham, ME).

Following NPC expansion, cells were collected and stained with the following antibodies: CD95 (BioLegend, San Diego, CA), HLA-DR (MHC-II) (BioLegend), CD80 (B7-1) ) (BioLegend), CD86 (B7-2) (BioLegend), and isotype-matched IgGs (Beckman Coulter, Fullerton, CA). Samples ( $n=3)$ of each expanded NPC group, $3 \times 10^{5}$ expanded cells were incubated on ice in cold PBS containing 0.1\% Chrom-Pure Human IgG whole molecule (Jackson ImmunoResearch Laboratories, West Grove, PA) and 1\% NaN3 (Sigma-Aldrich) for $30 \mathrm{~min}$, followed by incubation in the dark in the primary antibody for $30 \mathrm{~min}$. Fluorescence signals were analyzed using a BD FACS LSRFORTESSA ${ }^{\text {TM }}$ flow cytometer and FCS Express 4 software package (De Novo Software, Los Angeles, CA).Twenty thousand events of each sample were collected using CellQuest Pro software (BD Biosciences) and analyses performed using FCS Express 4 software package (DeNovo Software).

For co-culture and proliferation analysis, the same flow cytometry staining procedure as above was used. However, following IRB approval, peripheral blood mononuclear cells (PBMCs) were harvested from $20 \mathrm{~mL}$ of whole blood (26-year-old healthy male), and separated by density 
centrifugation for 40 minutes at $300 \mathrm{~g}$ in $50 \mathrm{~mL}$ conical tubes using Ficoll-Paque PLUS (GE Healthcare Life Sciences, Marlborough, MA). The PBMC layer was collected, washed with PBS, counted for viability using Trypan Blue staining and hemocytometer, and added at a 10:1 ratio $(0.2$ x $10^{6} \mathrm{PBMCs} /$ well $)$ in co-culture with expanded NPCs $\left(0.2 \times 10^{5} /\right.$ well $)$ in cell culture grade $96-$ well plates (Corning ${ }^{\oplus}$, Corning, NY) for 3 days. NPCs were allowed to attach to wells 24 hours prior to addition of PBMCs for co-culture. Cell culture media for this stage of experiments was RPMI-1640 with HEPES and L-glutamine (ThermoFisher, USA). Positive proliferation control consisted of stained PBMCs with Concanavalin A from Canavalia ensiformis (Sigma-Aldrich, USA) at $[10 \mathrm{ug} / \mathrm{mL}]$ added to media. PBMCs were stained with CellVue ${ }^{\circledR}$ Claret according to manufacturer's instructions prior to co-culturing. After 3 days of co-culture with expanded NPCs, PBMCs were collected from each well and stained with the following antibody cocktail: CD3, CD4, CD8, and CD45, and LIVE/DEAD® Fixable Red Dead Cell Stain Kit (Invitrogen, Life Technologies) per manufacturer instructions, and were collected and measured using a BD FACS LSRFORTESSA $^{\text {TM }}$ flow cytometer (BD Biosciences). Twenty thousand events of each sample were collected using CellQuest Pro software (BD Biosciences); cell proliferation index was analyzed using ModFit LT ${ }^{\mathrm{TM}}$ version 3.1 (Verity Software House) and analyses performed using FCS Express 4 software package (DeNovo Software).

\section{Redifferentiation induction and evaluation}

Expanded cells $\left(3 \times 10^{5}\right)$ were centrifuged at $500 \mathrm{~g}$ for $5 \mathrm{~min}$ in a $15-\mathrm{mL}$ polypropylene tube to form a pellet. After overnight incubation (day 0), the pellets were cultured in a serum-free chondrogenic medium consisting of high-glucose Dulbecco's Modified Eagle's Medium (DMEM), $40 \mu \mathrm{g} / \mathrm{mL}$ proline, $100 \mathrm{nM}$ dexamethasone, $100 \mathrm{U} / \mathrm{mL}$ penicillin, $100 \mu \mathrm{g} / \mathrm{mL}$ streptomycin, $0.1 \mathrm{mM} \mathrm{L}$ ascorbic acid-2-phosphate, and 1 $\times$ ITS ${ }^{\text {TM }}$ Premix (BD Biosciences) with the supplementation of 10 
$\mathrm{ng} / \mathrm{mL}$ transforming growth factor beta3 (TGFß3, PeproTech, Inc.) in a 5\% $\mathrm{O}_{2}$ incubator as long as 21 days. The pellets were evaluated for NP cell redifferentiation using pellet size, biochemical analysis, and quantitative real-time polymerase chain reaction (qPCR).

Representative pellets ( $\mathrm{n}=4$ ) were digested at $60^{\circ} \mathrm{C}$ for $4 \mathrm{~h}$ with $125 \mu \mathrm{g} / \mathrm{mL}$ papain in PBE buffer (100 mM phosphate, $10 \mathrm{mM}$ ethylenediaminetetraacetic acid, $\mathrm{pH}$ 6.5) containing $10 \mathrm{mM}$ cysteine. To quantify cell density, the amount of DNA in the papain digestion was measured using the QuantiT $^{\text {TM }}$ PicoGreen ${ }^{\circledR}$ dsDNA assay kit (Life Technologies) with a CytoFluor ${ }^{\circledR}$ Series 4000 (Applied Biosystems, Foster City, CA). GAG was measured using dimethylmethylene blue (DMMB) dye and a Spectronic ${ }^{\mathrm{TM}}$ BioMate $^{\mathrm{TM}} 3$ Spectrophotometer (Thermo Fisher Scientific, Waltham, MA) with bovine chondroitin sulfate (Sigma-Aldrich) as a standard.

Total RNA was extracted from representative samples $(n=4)$ using an RNase-free pestle in TRIzol ${ }^{\circledR}$ (Life Technologies). Two micrograms of mRNA were used for reverse transcriptase with HighCapacity cDNA Archive Kit (Applied Biosystems) at $37^{\circ} \mathrm{C}$ for $120 \mathrm{~min}$. NP cell redifferentiation related genes: type II collagen (COL2A1) (Assay ID Hs00156568_m1) and paired box protein 1 (PAXI) (Assay ID Hs01071291_g1), were customized by Applied Biosystems as part of their Custom TaqMan ${ }^{\circledR}$ Gene Expression Assays. Glyceraldehyde-3-Phosphate Dehydrogenase (GAPDH) (Assay ID Hs02758991_g1) was carried out as the endogenous control gene. TaqMan ${ }^{\circledR}$ real-time PCR was performed with the iCycler $\mathrm{iQ}^{\mathrm{TM}}$ Multi Color Real-Time PCR Detection and calculated by computer software (Perkin-Elmer, Wellesley, MA). Relative transcript levels were calculated as $\chi^{-} 2^{-\Delta \Delta \mathrm{Ct}}$, in which $\Delta \Delta \mathrm{Ct}=\Delta \mathrm{E}-\Delta \mathrm{C}, \Delta \mathrm{E}=\mathrm{Ct}_{\exp }-\mathrm{Ct}_{\mathrm{GAPDH}}$, and $\Delta \mathrm{C}=\mathrm{Ct}_{\mathrm{ct} 1}-\mathrm{Ct}_{\mathrm{GAPDH}}$. 


\section{Statistical Analysis}

The three response variables of interest (Viable, CD4+ and CD8+) assume the form of proportions that are relatively close to zero. It is well known that is such cases variances between groups are non-homogeneous. For small samples homogeneity of variance is a key assumption associated with ANOVA models. In order to stabilize the variances we used the arcsin of the square root of the measured proportions in an Analysis of Variance (ANOVA) to determine significance. In all cases the results were deemed to be significant if the resulting p-value was $<.05$. All analyses were done using JMP V12.2 (SAS Institute, Cary NC). The ANOVA model included two factors: One factor (Donor) was a blocking or control factor while the other (ECM Type) was the experimental factor. ECM type had 3 levels: Plastic, Allo-ECM and XenoECM. When the effect of ECM type was significant, pairwise difference between the three levels were explored using Tukey's HSD procedure. 


\section{Results}

dECM expansion increases NPC proliferation

In proliferation analyses, all dECM expansion conditions were superior in terms of proliferation index (PI) to Plastic group (Fig 1). Allo-ECM and Xeno-ECM exhibited the greatest proliferation indices, at 14.94 (1.99-fold vs. Plastic) and 14.59 (1.95-fold vs. Plastic), respectively. Additionally, all dECM groups exhibited peak generations at Generation 4 or Generation 5, whereas Plastic exhibited the greatest peak at Generation 3.

dECM conditions do not elicit differences in immune responses

In immunophenotyping experiments, all groups exhibited insignificant differences compared to Plastic, with no measurable increases in HLA-DR, CD80, or CD86 expression following dECM expansion (Fig 2). Despite similar percentages of CD95+ (Fas) cell expression to Plastic following dECM expansion, Plastic exhibited the greatest Mean Fluorescence Intensity (MFI) versus all other groups, with Plastic MFI = 704.64; AutoECM = 565.58; AlloECM = 606.88; and XenoECM = 529.86 (Fig 3). In co-culture experiments where expanded NPCs and PBMCs were cultured together for 72 hours, no significant increases in the percentage of proliferated PBMCs were observed between Plastic and either AlloECM or XenoECM groups in T-helper cells (CD45+/CD3+/CD4+) (Fig 4C), or in proliferation analysis of viable PBMC population (CD45+)

(Fig 4B). A small decrease was measured in the percentage of proliferation in cytotoxic T-cells (CD45+/CD3+/CD8+) in Xeno-ECM versus Plastic (Fig 4D). Robust PBMC proliferation was measured in the ConA stimulated positive control, with $93.45 \%$ proliferation occurring (Fig 4A), whereas co-culture proliferation did not exceed $3.5 \%$ in any group. 
At Day 14, Plastic pellets exhibited rather irregular shapes, compared to the larger, spherical AlloECM and XenoECM groups (Fig 5A). The pellet size of AlloECM and XenoECM were both marginally larger than Plastic at both Day 14 and Day 28 of redifferentiation. Day 14 AlloECM and XenoECM pellets were similar in size and shape to the Plastic pellet at Day 28 (Fig 5A). Both Allo-ECM and Xeno-ECM increased DNA ratio versus Plastic group at Day 14 and Day 28 of redifferentiation (Fig 5B). Additionally, Allo-ECM and Xeno-ECM both exhibited the greatest glycosaminoglycan (GAG) content, significantly greater than Plastic at both Day 14 and Day 28 (Fig 5B), and exhibited trends toward greater GAG/DNA ratios at Day 28 of redifferentiation (Fig 5B). Real-time qPCR revealed similar levels of PAX1 mRNA expression across all groups; however, both AlloECM and XenoECM exhibited an approximately two-fold significant increase in COL2A1 mRNA (Fig 5C). 


\section{Discussion}

In this study, we compared the immunological properties of dECM, prepared from different donor sources, and their ability to expand human nucleus pulposus cells harvested from herniated lumbar discs. To our knowledge, there are no other studies which have compared dECM expansion of human lumbar NP cultures for immunogenicity via PBMC proliferation. For the first time, we showed that dECM, regardless of source, did not significantly increase HLA-DR, CD80, CD86, or CD95 expression (Fig 2 \& 3). In a previous study, we showed that expansion of NPCs on an adipose-derived allogeneic dECM could decrease CD95 expression [23], but did not observe this result in the current study. There may be differences in the immunomodulatory properties dECMs based upon cell type and source, however, this hypothesis warrants further investigation and evidence. Additionally, when dECM expanded cells were co-cultured with PBMCs, no increases in proliferation of T-cell or viable PBMC populations were observed (Fig 4). The results of this study are similar to those published by Stich and colleagues, who used human cervical NPCs cultured in 3D fibrin/poly (lactic-co-glycolic) acid transplants and tested the proliferation of CFSE-labeled PBMCs [13]; however, unlike the results from their study, we did not measure any elevated proliferation responses following 3D-culture ( $\mathrm{dECM}$ in the case of our study). They suggested that their result could be attributed to potential unidentified stimuli from the cells or matrix [13]. Overall, we assume there is low immunogenicity in our own cultures which utilized dECM expansion of NPCs.

In a previous study, it has been shown that xenogeneic, but not allogeneic dECM expansion of synovial-derived stem cells could increase HLA-DR expression [22], raising potential questions regarding dECMs' effects on expanded cell immunogenicity with dECM source/composition representing a potential immunogenic stimuli. Stich and colleagues reported low HLA-DR 
expression in NPCs, however, did observe a small increase in more severely degenerated NPCs [13]. In the current study, we did not observe any measurable HLA-DR expression, or any increase in HLA-DR expression, regardless of dECM expansion condition (Fig 2). Furthermore, in the current study, we report the same result for CD80 and CD86, co-stimulatory ligands for T-helper cell responses [32]. Recent literature has shown that blockade of CD80 and CD86 co-stimulatory signals can result alloantigen-specific anergy of T-cells [33]. Interestingly, it has been suggested that lack of expression of these co-stimulatory molecules could partially explain the lack of immune response to tumor cells [34], and may also benefit cell-based therapies targeting IVDs.

Although the results of this study are promising for the future therapeutic applications for dECM expansion of human NPCs, we recognize several limitations of the study. These limitations include NP and blood donor numbers and overall lack of dECM characterization. Also, future detection of HLA-ABC (MHC-I) may be useful to provide more complete immunoprofiles and supporting data for T-cyotoxic cell interactions. Studies that more fully validate this model are necessary, especially those incorporating more sophisticated in vivo models for immune rejection of dECMexpanded NPCs, and would vastly improve and support the current findings. Also, despite emerging literature implicating degenerative status of NPCs [13] [23], there is still an overall lack of literature investigating the predisposition of degenerative status and its effect on the cells' response to preconditioning strategies; therefore, it may be useful to compare the efficacy of various preconditioning methods and resulting immunogenicity in future studies. Overall, dECMs, particularly those from allogeneic and xenogeneic sources could serve as a potential source of commercially available, effective, and safe means of human NPC expansion for future utilization in cell-based therapies. 


\section{Acknowledgements}

We thank Dr. Gerry Hobbs for his assistance in the statistical analysis. We also thank Suzanne Danley for editing the manuscript. No competing financial interests exist. 


\section{References}

[1] Hoy D, Bain C, Williams G, March L, Brooks P, Blyth F, Woolf A, Vos T, Buchbinder R. A systematic review of the global prevalence of low back pain. Arthritis Rheum. 2012 Jun;64(6):2028-37.

[2] Walsh NE, Brooks P, Hazes JM, Walsh RM, Dreinhöfer K, Woolf AD, Akesson K, Lidgren L; Bone and Joint Decade Task Force for Standards of Care for Acute and Chronic Musculoskeletal Pain.. Standards of care for acute and chronic musculoskeletal pain: the Bone and Joint Decade (2000-2010). Arch Phys Med Rehabil. 2008 Sep;89(9):1830-45.

[3] Ito K, Creemers L. Mechanisms of intervertebral disk degeneration/injury and pain: a review. Global Spine J. 2013 Jun;3(3):145-52.

[4] Pennicooke B, Moriguchi Y, Hussain I, Bonssar L, Härtl R. Biological Treatment Approaches for Degenerative Disc Disease: A Review of Clinical Trials and Future Directions. Cureus. 2016 Nov 22;8(11).

[5] Tong W, Lu Z, Qin L, Mauck RL, Smith HE, Smith LJ, Malhotra NR, Heyworth MF, Caldera F, Enomoto-Iwamoto M, Zhang Y. Cell therapy for the degenerating intervertebral disc. Transl Res. 2017 Mar;181:49-58.

[6] Gunzburg R, Parkinson R, Moore R, Cantraine F, Hutton W, Vernon-Roberts B, Fraser R. A cadaveric study comparing discography, magnetic resonance imaging, histology, and mechanical behavior of the human lumbar disc. Spine (Phila Pa 1976). 1992 Apr;17(4):417-26.

[7] Sakai D, Grad S. Advancing the cellular and molecular therapy for intervertebral disc disease. Adv Drug Deliv Rev. 2015 Apr;84:159-71.

[8] Pei M. Environmental preconditioning rejuvenates adult stem cells' proliferation and chondrogenic potential. Biomaterials. 2017 Feb;117:10-23.

[9] Zhang Y, Li J, Davis ME, Pei M. Delineation of in vitro chondrogenesis of human synovial stem cells following preconditioning using decellularized matrix. Acta Biomater. 2015 Jul;20:39-50.

[10] Lynch K, Pei M. Age associated communication between cells and matrix: a potential impact on stem cell-based tissue regeneration strategies. Organogenesis. 2014;10(3):289 98.

[11] He F, Pei M. Rejuvenation of nucleus pulposus cells using extracellular matrix deposited by synovium-derived stem cells. Spine (Phila Pa 1976). $2012 \mathrm{Mar}$ 15;37(6):459-69.

[12] Croutze R, Jomha N, Uludag H, Adesida A. Matrix forming characteristics of inner and outer human meniscus cells on 3D collagen scaffolds under normal and low oxygen 
tensions. BMC Musculoskelet Disord. 2013 Dec 13;14:353.

[13] Stich S, Stolk M, Girod PP, Thomé C, Sittinger M, Ringe J, Seifert M, Hegewald AA. Regenerative and immunogenic characteristics of cultured nucleus pulposus cells from human cervical intervertebral discs. PLoS One. 2015 May 19;10(5):e0126954.

[14] Kaneyama S, Nishida K, Takada T, Suzuki T, Shimomura T, Maeno K, Kurosaka M, Doita M. Fas ligand expression on human nucleus pulposus cells decreases with disc degeneration processes. J Orthop Sci. 2008 Mar;13(2):130-5.

[15] Fu X, Yang H, Zhang H, Wang G, Liu K, Gu Q, Tao Y, Chen G, Jiang X, Li G, Gu Y, Shi Q. Improved osteogenesis and upregulated immunogenicity in human placentaderived mesenchymal stem cells primed with osteogenic induction medium. Stem Cell Res Ther. 2016 Sep 20;7(1):138.

[16] Mousavinejad M, Andrews PW, Shoraki EK. Current Biosafety Considerations in Stem Cell Therapy. Cell J. 2016 Jul-Sep;18(2):281-7.

[17] Watschinger B. Indirect recognition of allo MHC peptides--potential role in human transplantation. Nephrol Dial Transplant. 1999 Jan;14(1):8-11.

[18] Stickel N, Hanke K, Marschner D, Prinz G, Köhler M, Melchinger W, Pfeifer D, Schmitt-Graeff A, Brummer T, Heine A, Brossart P, Wolf D, von Bubnoff N, Finke J, Duyster J, Ferrara J, Salzer U, Zeiser R. MicroRNA-146a reduces MHC-II expression via targeting JAK/STAT-signaling in dendritic cells after stem cell transplantation. Leukemia. 2017 May 9.

[19] Vokaer B, Charbonnier LM, Lemaître PH, Spilleboudt C, Le Moine A. IL-17A and IL 2-expanded regulatory $\mathrm{T}$ cells cooperate to inhibit Th1-mediated rejection of MHC II disparate skin grafts. PLoS One. 2013 Oct 11;8(10):e76040.

[20] Fang Y, Wang B, Zhao Y, Xiao Z, Li J, Cui Y, Han S, Wei J, Chen B, Han J, Meng $\backslash$ Q, Hou X, Luo J, Dai J, Jing Z. Collagen scaffold microenvironments modulate cell lineage commitment for differentiation of bone marrow cells into regulatory dendritic cells. Sci Rep. 2017 Feb 7;7:42049.

[21] Yuan T, Luo H, Tan J, Fan H, Zhang X. The effect of stress and tissue fluid microenvironment on allogeneic chondrocytes in vivo and the immunological properties of engineered cartilage. Biomaterials. 2011 Sep;32(26):6017-24.

[22] Zhang Y, Pizzute T, Li J, He F, Pei M. sb203580 preconditioning recharges matrixexpanded human adult stem cells for chondrogenesis in an inflammatory environment - A feasible approach for autologous stem cell based osteoarthritic cartilage repair.

Biomaterials. 2015 Sep;64:88-97. 
[23] Pizzute, Pei. Preconditioning strategies unequally impact regeneration of nucleus pulposus cells from human herniated discs: a proof of principle study. TBD. 2017.

[24] Takada T, Nishida K, Doita M, Kurosaka M. Fas ligand exists on intervertebral disc cells: a potential molecular mechanism for immune privilege of the disc. Spine (Phila Pa 1976). 2002 Jul 15;27(14):1526-30.

[25] Inui Y, Nishida K, Doita M, Takada T, Miyamoto H, Yoshiya S, Kurosaka M. Fasligand expression on nucleus pulposus begins in developing embryo. Spine (Phila Pa 1976). 2004 Nov 1;29(21):2365-9.

[26] Sun Z, Liu ZH, Chen YF, Zhang YZ, Wan ZY, Zhang WL, Che L, Liu X, Wang HQ, Luo ZJ. Molecular immunotherapy might shed a light on the treatment strategies for disc degeneration and herniation. Med Hypotheses. 2013 Sep;81(3):477-80.

[27] Ma CJ, Liu X, Che L, Liu ZH, Samartzis D, Wang HQ. Stem Cell Therapies for Intervertebral Disc Degeneration: Immune Privilege Reinforcement by Fas/FasL Regulating Machinery. Curr Stem Cell Res Ther. 2015;10(4):285-95.

[28] Imai Y, Kimura T, Murakami A, Yajima N, Sakamaki K, Yonehara S. The CED-4homologous protein FLASH is involved in Fas-mediated activation of caspase-8 during apoptosis. Nature. 1999 Apr 29;398(6730):777-85. Erratum in: Nature 1999 Jul 1;400(6739):89.

[29] Westendorf JJ, Lammert JM, Jelinek DF. Expression and function of Fas (APO1/CD95) in patient myeloma cells and myeloma cell lines. Blood. 1995 Jun 15;85(12):3566-76.

[30] Park JB, Lee JK, Park EY, Riew KD. Fas/FasL interaction of nucleus pulposus and cancer cells with the activation of caspases. Int Orthop. 2008 Dec;32(6):835-40.

[31] Chen SQ, Lin JP, Zheng QK, Chen SJ, Li M, Lin XZ, Wang SZ. Protective effects of paeoniflorin against FasL-induced apoptosis of intervertebral disc annulus fibrosus cells via Fas-FasL signalling pathway. Exp Ther Med. 2015 Dec;10(6):2351-2355.

[32] Lanier LL, O'Fallon S, Somoza C, Phillips JH, Linsley PS, Okumura K, Ito D, Azuma M. CD80 (B7) and CD86 (B70) provide similar costimulatory signals for T cell proliferation, cytokine production, and generation of CTL. J Immunol. 1995 Jan 1;154(1):97-105.

[33] Davies JK, Barbon CM, Voskertchian AR, Nadler LM, Guinan EC. Induction of alloantigen-specific anergy in human peripheral blood mononuclear cells by alloantigen stimulation with co-stimulatory signal blockade. J Vis Exp. 2011 Mar 14;(49). pii: 2673.

[34] Schultze J, Nadler LM, Gribben JG. B7-mediated costimulation and the immuneresponse. Blood Rev. 1996 Jun;10(2):111-27. 
Figure 5.1

A

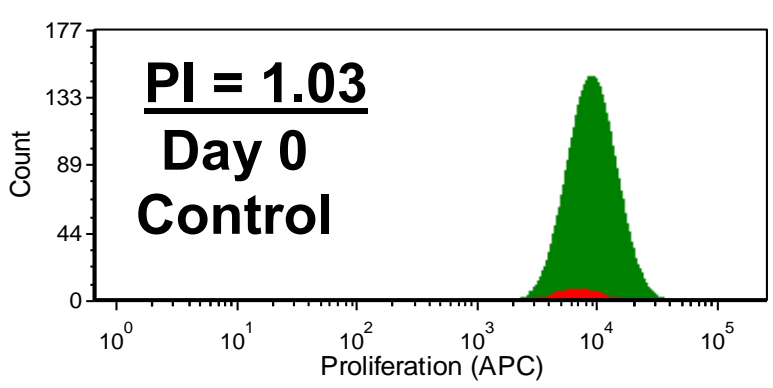

B

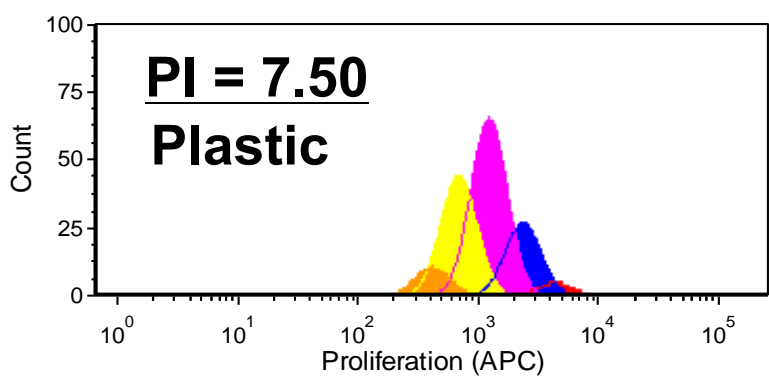

Cell Proliferation - Undivided Cells - Generation 1 Generation 2 $\square$ Generation 3

C

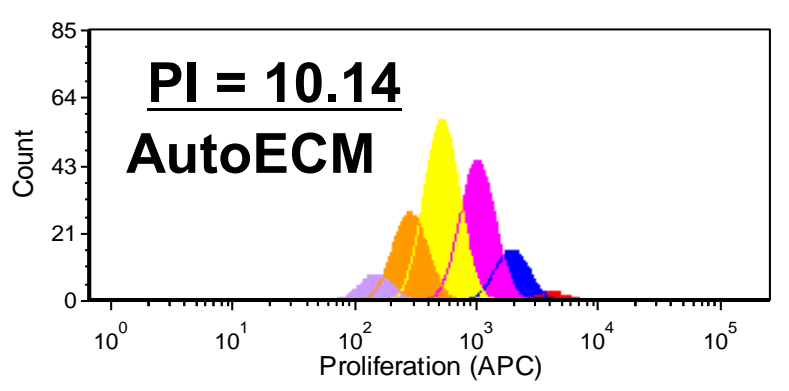

Cell Proliferation Undivided Cells Generation 1 Generation 2 Generation 3 $\square$ Generation 4 $\square$ Generation 5 $\square$ Generation 6

D

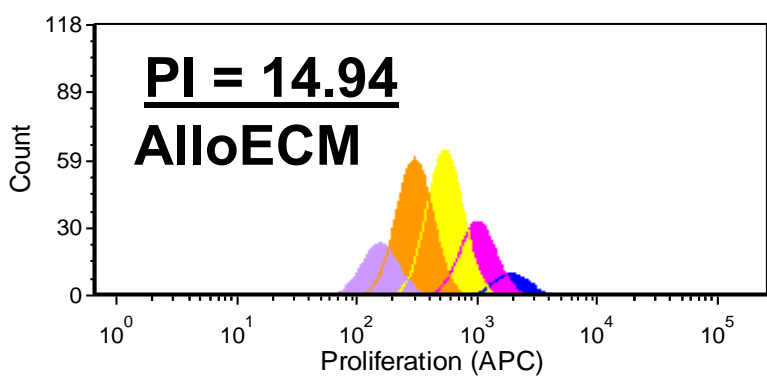

Cell Proliferation Undivided Cells - Generation 1

Generation 2 Generation 3 $\square$ Generation 4

$\square$ Generation 5 $\square$ Generation 6

E

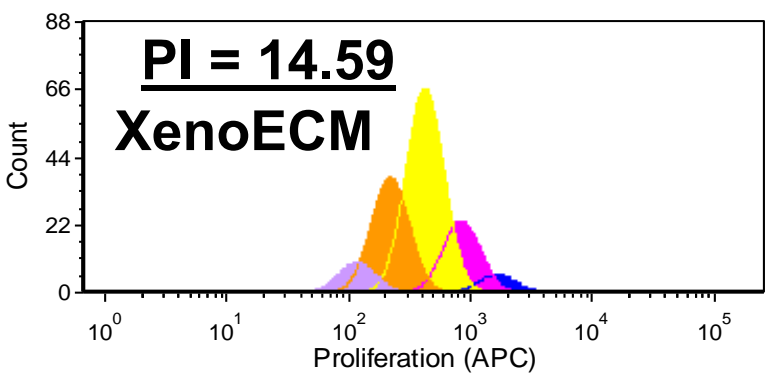

Cell Proliferation Undivided Cells Generation 1 Generation 2 Generation 3 Generation 4 $\square$ Generation 5 $\square$ Generation 6 
Figure 5.1: Proliferation of expanded NPCs. Claret red-labeled nucleus pulposus cells were measured via flow cytometry for their proliferation indices and generation analysis at Day 0 (A), and after 5 days on either Plastic (B), autologous dECM (C), allogeneic dECM (D), or xenogeneic $\operatorname{dECM}(\mathbf{E})$. 
Figure 5.2
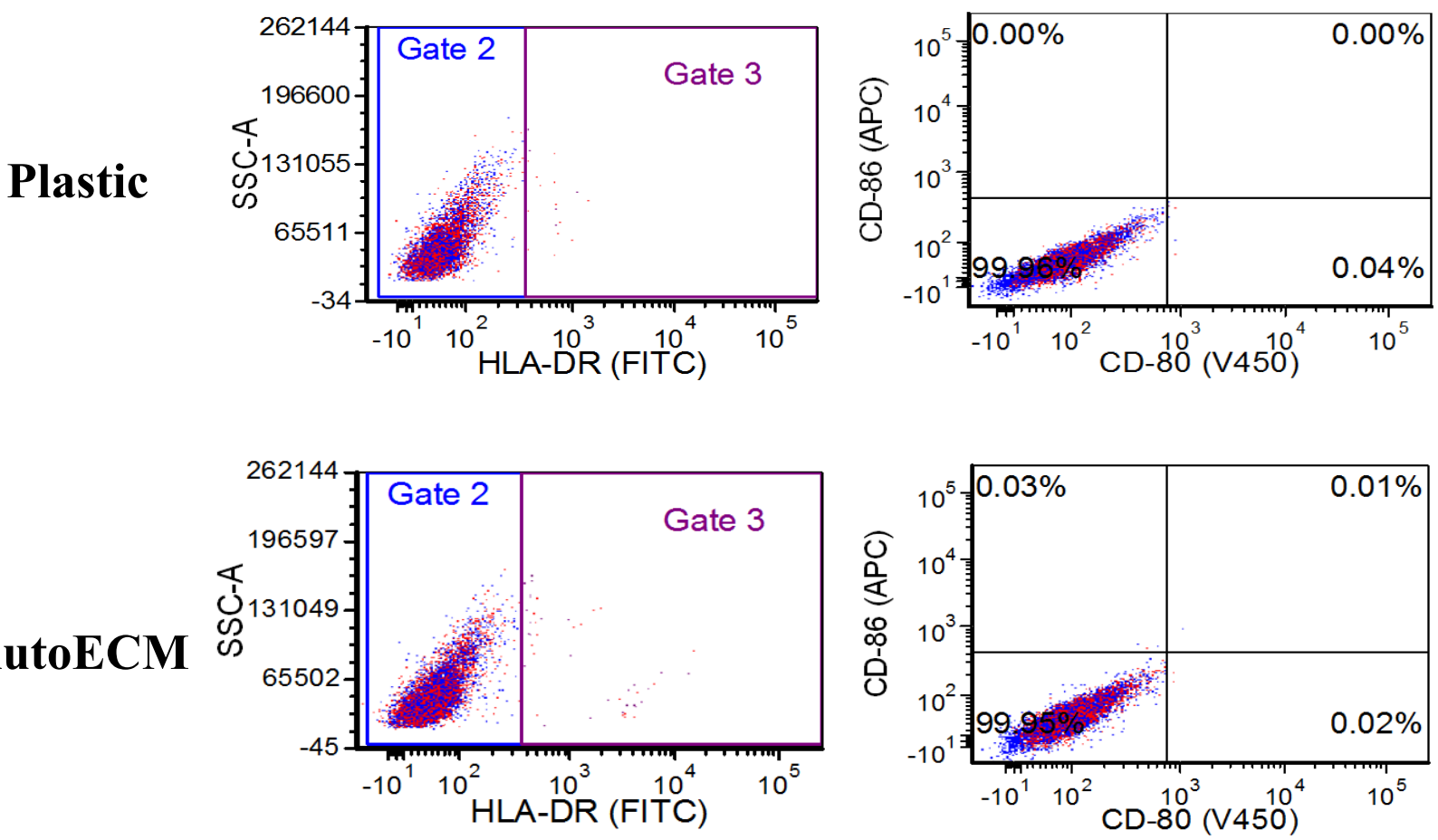

AutoECM
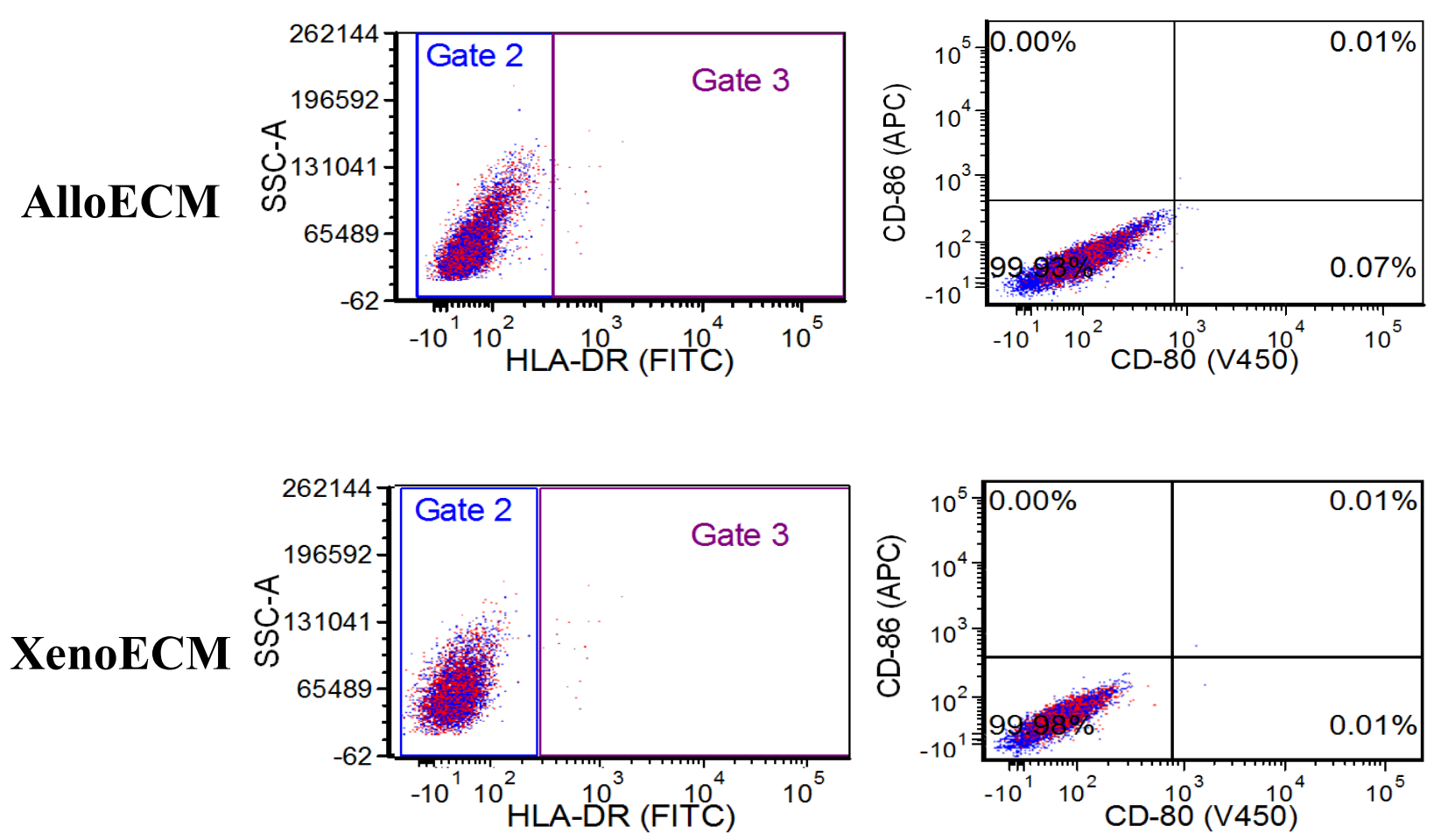
Figure 5.2: Immune marker expression following expansion. Flow cytometry analysis of immune surface marker HLA-DR (MHC-II) expression (left column), CD80 (B7-1) and CD86 (B7-2) expression (right column) for NPCs expanded on Plastic (A), AutoECM (B), AlloECM (C), and XenoECM (D). 
Figure 5.3
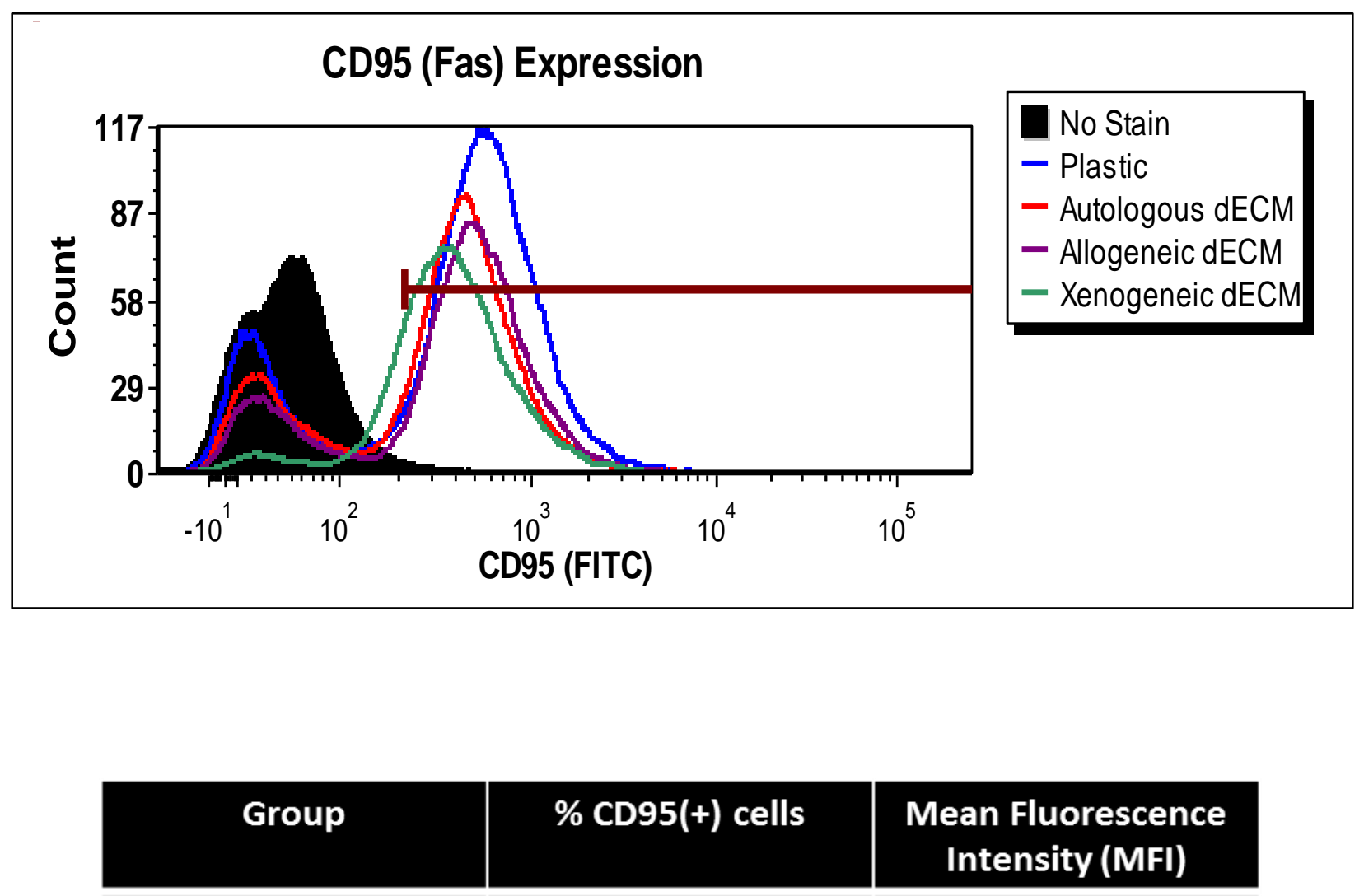

\section{\% CD95(+) cells}

Mean Fluorescence

Intensity (MFI)

Plastic

$74.90 \%$

704.64

AutoECM

$70.00 \%$

565.58

AlloECM

$75.37 \%$

606.88

XenoECM

$74.79 \%$

529.86 
Figure 5.3: Expression of CD95 (Fas) by expanded NPCs. Flow cytometry analysis of CD95 expression in a histogram (top), and in table format (bottom), depicting the percentage of CD95positve cells and mean fluorescence intensity (MFI) for each expansion condition. 
Figure 5.4

A

B

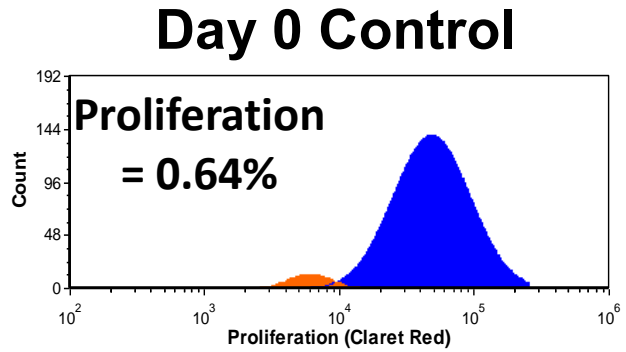

Positive Stain (CR

Generation 1

Generation 2

ConA Stimulated
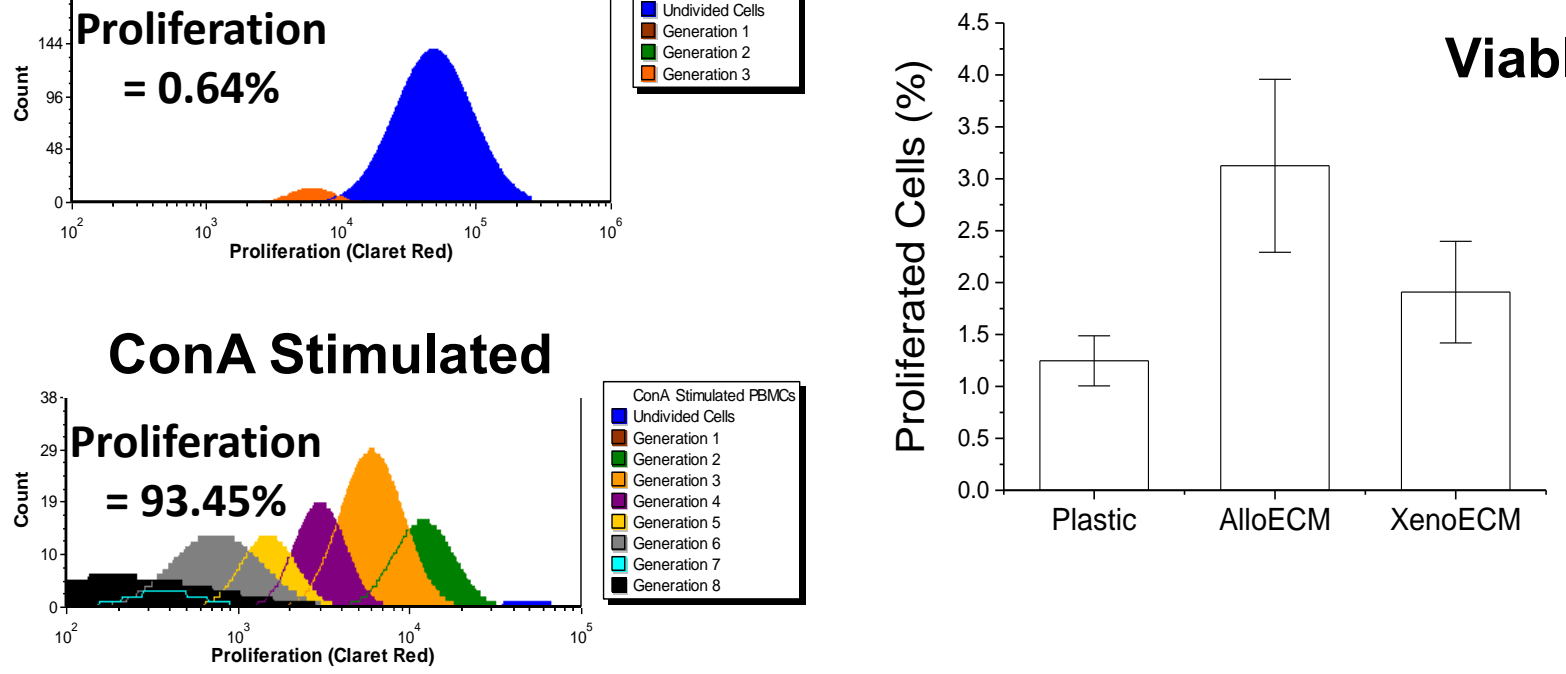

C

D
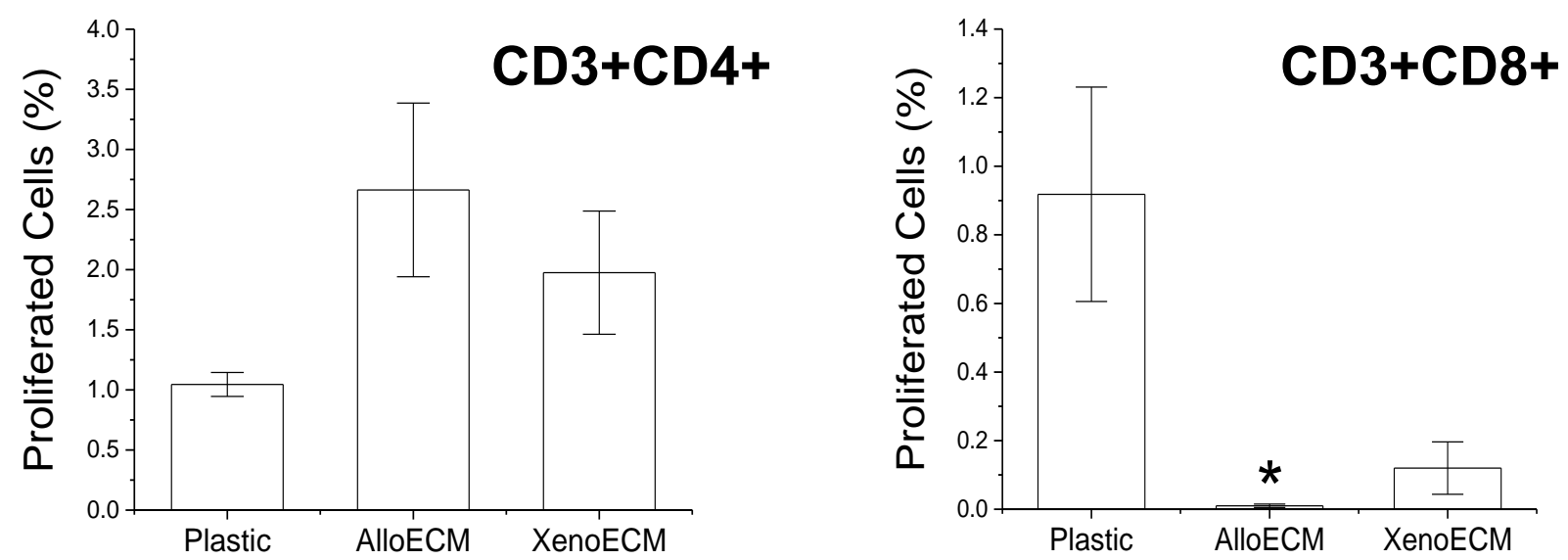
Figure 5.4: PBMC proliferation following co-culture with expanded NPCs. Stained-PBMCs were measured via flow cytometry for their generational and percent proliferation at Day 0, and after 3 days of ConA-stimulation as proliferation controls (A). Percent of proliferation of all viable (B), CD3+CD4+ (C), and CD3+CD8+ (D) PBMCs populations following 3 days in co-culture with NPCs expanded on Plastic, AlloECM, or XenoECM were also measured. 
Figure 5.5
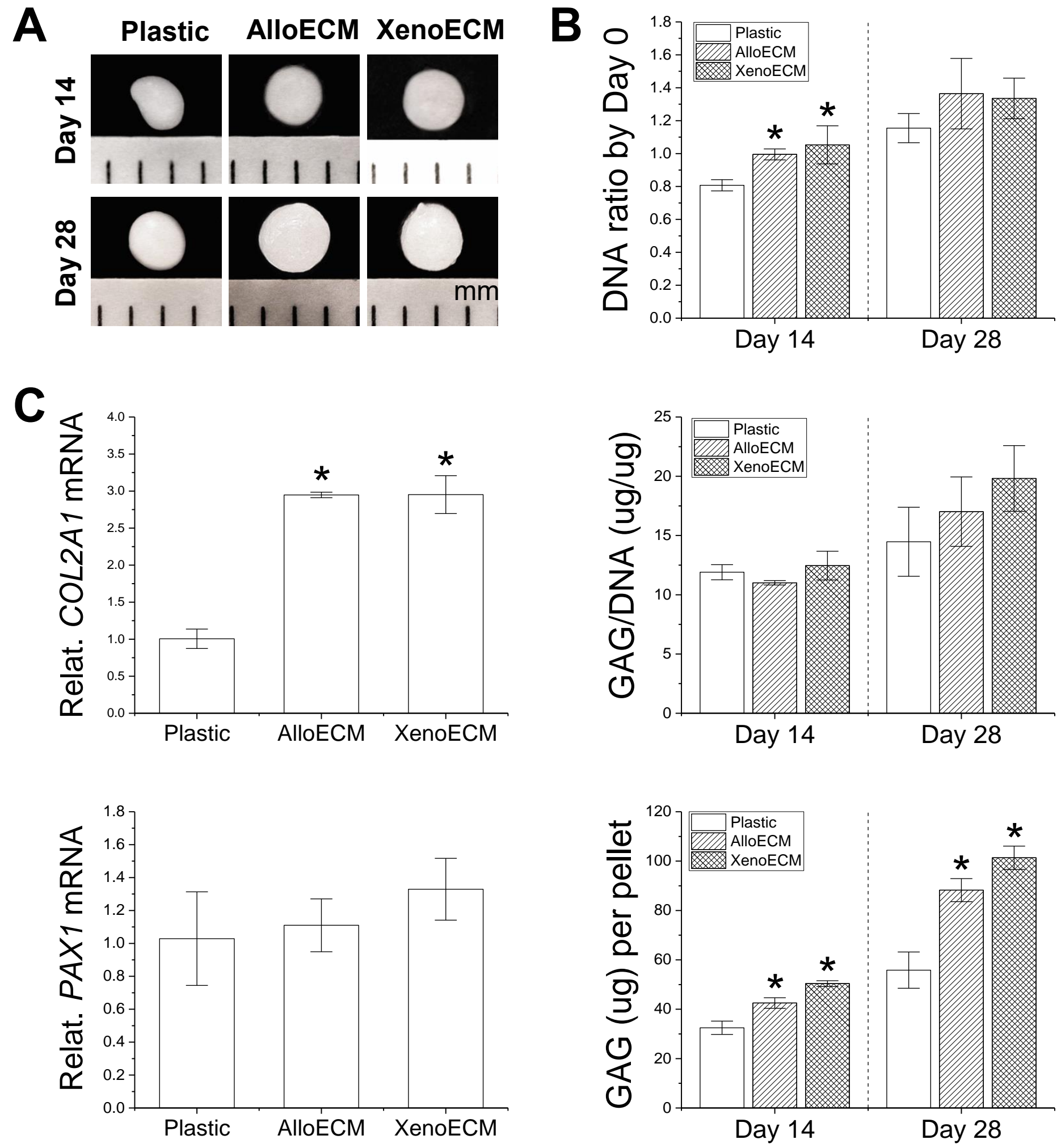
Figure 5.5: Redifferentiation of ECM expanded NPCs. Representative redifferentiated cell pellets, formed from each group of expanded NPCs were cultured and collected at Day 14 and Day 28 for comparison (A).GAG content, DNA, and GAG/DNA ratios were measured at both Day 14 and 28 for each group (B). Real-time PCR was used to detect the expression of COL2A1 and PAX1 in expanded and redifferentiated NPCs $(\mathbf{C})$. 
Chapter 6:

\section{General Discussion}




\section{Summary}

The primary objectives of this dissertation were to (1) investigate the roles of WNT3A, WNT5A, and WNT11 gene expression in NP redifferentiation, (2) evaluate the efficacy of dECM, FGF-2, and hypoxic preconditioning of human NPCs and SDSCs, (3) determine the redifferentiation and immunophenotypic changes of human NPCs following allogeneic and xenogeneic (porcine) dECM expansion, and (4) determine if allogeneic and xenogeneic (porcine) dECM expansion increased T-cell proliferation. The long-term goal of these studies is to provide new insights and data to assist in the development of effective cell preconditioning protocols for efficient and safe ex vivo expansion of human NPCs for intervertebral disc regeneration and repair. The central hypothesis of this work is that preconditioning methods such as dECM expansion and FGF-2 supplementation can be effectively utilized for harvested NPC expansion without apparent immunological concerns; furthermore, this could be done through the modulation of wnt signals to improve redifferentiation potential. By understanding the downstream effects of preconditioning strategies on NPC rejuvenation, as well as the upstream biochemical signaling pathways at work during NPC redifferentiation, mindful and effective approaches can be developed for cell-based IVD regeneration.

In this dissertation, we demonstrated that non-canonical Wnt signals, Wnt5A and Wnt11, are important regulators of NPC redifferentiation, and along with canonical Wnt3A, these signals appear to be stage-specifically expressed regulators of SDSC chondrogenesis when preconditioned with FGF-2 (Pizzute et al., 2016). Furthermore, we demonstrated that preconditioning of human NPCs by allogeneic and xenogeneic dECM, as well as FGF-2 supplementation in both SDSCs and NPCs can be effectively utilized to increase proliferative and redifferentiation potential. These dECM conditions did not elicit apparent increases in the expression of potentially disadvantageous 
immunomarkers (HLA-DR, CD80, CD86, or CD95), nor do they cause increases proliferation of viable PBMCs, $\mathrm{CD} 3+\mathrm{CD} 4+\mathrm{T}$-cells, or CD3+CD8+ T-cells in co-culture with dECM-expanded substrates. Overall, dECM and FGF-2 preconditioning methods, as well as the proper modulation of Wnt signaling pathways during NPC expansion each hold promising outlook and potential benefits for future cell-based therapies in IVD repair and orthopaedic interventions.

\section{Future Directions}

\section{Detecting of potential immune issues in cultures}

Despite the known potential benefits of various preconditioning strategies for cell culture [1], it is imperative that these cells do not elicit immune responses when introduced or reintroduced to a patient. For this reason, more studies are needed to fully evaluate the immunophenotypic changes in preconditioned cells and to perform the necessary experiments to ultimately elucidate the immune-related benefits and caveats of each preconditioning strategy. One of the initial experiments for assaying immune changes should include flow cytometry for immune surface marker analysis following preconditioning, particularly HLA-DR, complemented with T-cell costimulatory markers CD80 and CD86, as well as apoptosis-related markers CD95 (Fas) and CD178 (FasL), implicated in intervertebral disc immunity [2] [3]. Furthermore, studies investigating the relationships between preconditioning and Fas-mediated apoptosis could uncover new, clinically relevant results for future therapeutic applications in intervertebral disc regeneration. To date, few studies have compared the immunomodulatory properties of various preconditioning methods in a single well-controlled study. One common approach to model potential immune response to preconditioned cells is an MLR assay using labeled immune cells, typically isolated from a blood sample. Several groups have used a version of this technique to test 
preconditioned cells [4] [5]. Notably, there is a great demand for in vivo studies to investigate the role of preconditioned cells for safe intervertebral disc tissue repair, especially those that can further evaluate the effects of preconditioning-induced changes in immune characteristics. It is reasonably suspected that one preconditioning strategy is unlikely to be most superior or ideal for all tissues in terms of immunomodulation, especially since various tissues are dominated by distinct immunoregulatory factors and different immune cells [6]. With clinical applications in mind, an ideal preconditioning method or combination strategy should consider the multifaceted and specific nature of the immune reactions present in a particular target tissue, as well as the potentially altered immune properties of the preconditioned cell population.

\section{Identification of dECM properties}

In this dissertation and in past studies from our laboratory, we have thoroughly studied and demonstrated the ability of various dECMs to provide a useful cell expansion substrate for NPCs and MSCs alike [7] [8] [9] [10]. These dECMs can cause increased cell proliferation, differentiation potentials, and overall cell rejuvenation. Despite the benefits of dECM expansion, there is an abundance of unanswered research questions surrounding its use, as well as potential factors influencing its efficacy as a cell expansion strategy.

Some of the areas currently under investigation in our laboratory are the roles of various laminin subunits in nucleus pulposus cell, which have been shown to be important in nucleus pulposus ECM interactions [11] [12] [13]. Similar to the Wnt signaling studies presented in this dissertation, lentiviral transduction of NPCs using CRISPR-Cas9 vectors offers a powerful and accurate means to knockout specific laminin subunits, targeting the $L A M A 1, L A M B 1$, and $L A M C 1$ genes to study dECM interactions. We have been able to efficiently generate knockouts of these genes and 
validate them by immunofluorescence microscopy (Fig 6.1). After generating stable cell lines, it will be possible to create laminin-rich, and laminin-deficient dECMs (Fig 6.2) for direct comparison in proliferative consequences, redifferentiation potential, and immune-related issues associated with dECM-expanded NPC and stem cell populations. Additionally, it may be useful to measure and more comparatively study the differences in the physical properties of these dECMs, such as elasticity/stiffness [14] (Fig 6.3), and in the future, observe characterize the differences in these properties following structural modifications like those facilitated by the knockout of structural proteins like laminins. Ideally, future dECM applications and research studies will consider facets of ECM, including both form and function; this will ensure researchers and clinicians can develop the most effective and conscientious preconditioning strategies and cellbased approaches.

\section{Looking Ahead and Clinical Integration}

In addition to the physical and immunomodulatory properties of $\mathrm{dECM}$, there are other concerns to be addressed before these preconditioning techniques can be appropriately implemented for clinical use. In 2014, Alan Tyndall published his opinions and assessments regarding the current state of MSC research and therapeutics, highlighting many noteworthy concerns in the field [15]. Some of the resonating concerns presented in this article include a certain level skepticism surrounding the optimistic outcomes presented in current MSC literature and the administration of MSC-based interventions, which seem to exceed the current scientific knowledge [15][16]. Also, the seemingly short-lived immunomodulatory properties of MSCs themselves, experimental designs utilizing heterogenous and undefined cell populations, and perhaps most importantly, the overall lack of conclusiveness and continuity in the field surrounding MSC treatments are other 
areas for concern [15]. Additionally, Tyndall makes propositions involving the future adherence and consideration of safety and current good manufacturing practices (cGMP) for future MSC applications [15]. Interestingly, many of the perspectives and concerns raised by the author of this article are relevant to the development and use of preconditioning strategies, and could provide direction for future applications of dECM in a clinical environment.

Although it may be agreeable to propose that desirable immunomodulatory effects provided by MSCs may be short-lived as Tyndall suggests [15], in the case of dECM expansion and the use of $\mathrm{dECM}$ as a preconditioning strategy for IVD repair, the goal is likely better focused on the preservation of immune tolerance, rather than directing immunomodulatory applications via expanded cells. It seems that MSC applications with immunomodulatory capabilities may hold greater promise and sustainable solutions for chronic inflammatory and immunoregulatory conditions, such as rheumatoid and osteoarthritis [17], as opposed to IVD repair, which is not specifically defined by these characteristics. The results of the studies outlined in this dissertation have shown that dECM-expanded NPCs do not increase adverse immune proliferation of T-cells in co-cultures, nor do they increase CD95, HLA-DR, CD80, or CD86 expression. From our perspective, it seems that the real utility of $\mathrm{dECM}$ would be increased proliferation and redifferentiation capacity of expanded NPCs, and ultimately increased cellularity of damaged tissues, without inducing adverse immune effects. It is likely that other strategies or combination therapies, in conjunction with $\mathrm{dECM}$, would be more useful for long-term or sustainable immunomodulation. Additionally, well controlled studies which compare the immunomodulatory properties of defined cell populations for in situ intervertebral disc repair would provide useful insights to this concept. 
Points raised by Tyndall about the use of defined cell populations and cGMP however, hold much greater value, relevance, and are certainly applicable to the design, manufacturing, and utility of dECM as a clinical tool. By defining the cell populations used in the production of dECM coatings, the components likely have greater potential for standardization and control for future therapeutic modifications. Since there are many choices in terms of cell type in which dECM coatings can be derived and produced, choosing the most appropriate dECM type would seem like an obvious and important consideration. Our studies have suggested that the most appropriate $\mathrm{AECM}$ is the one that provides the best microenvironment for cell rejuvenation and directed differentiation successes in specific cell types [1][8][9][10]. This is most effectively aligned with our review, discussing that the most appropriate tissue specific stem cells for MSC therapies are those derived from the same tissue origins [18]; however, to date, studies comparing dECMs created from defined, well-characterized, and standardized cell lines, or those manufactured under cGMP remains unexplored. It should also be noted that the effect of degenerative status in preconditioning has yet to be extensively compared in IVD applications and for NPCs directly. Likewise, the differences in degenerative status and influence of harvested cells' overall condition on dECM composition (for $\mathrm{dECM}$ production) has yet to be studied. Also, as previously stated and in stride with the concerns for undue optimism in the future of MSC-based therapies [15], in vivo studies providing evidence and support for dECM applications as a vehicle for successful tissue repair are necessary. The next stage for dECM studies should be mindful of these considerations to most effectively progress the model from the lab to clinic. 
Figure 6.1
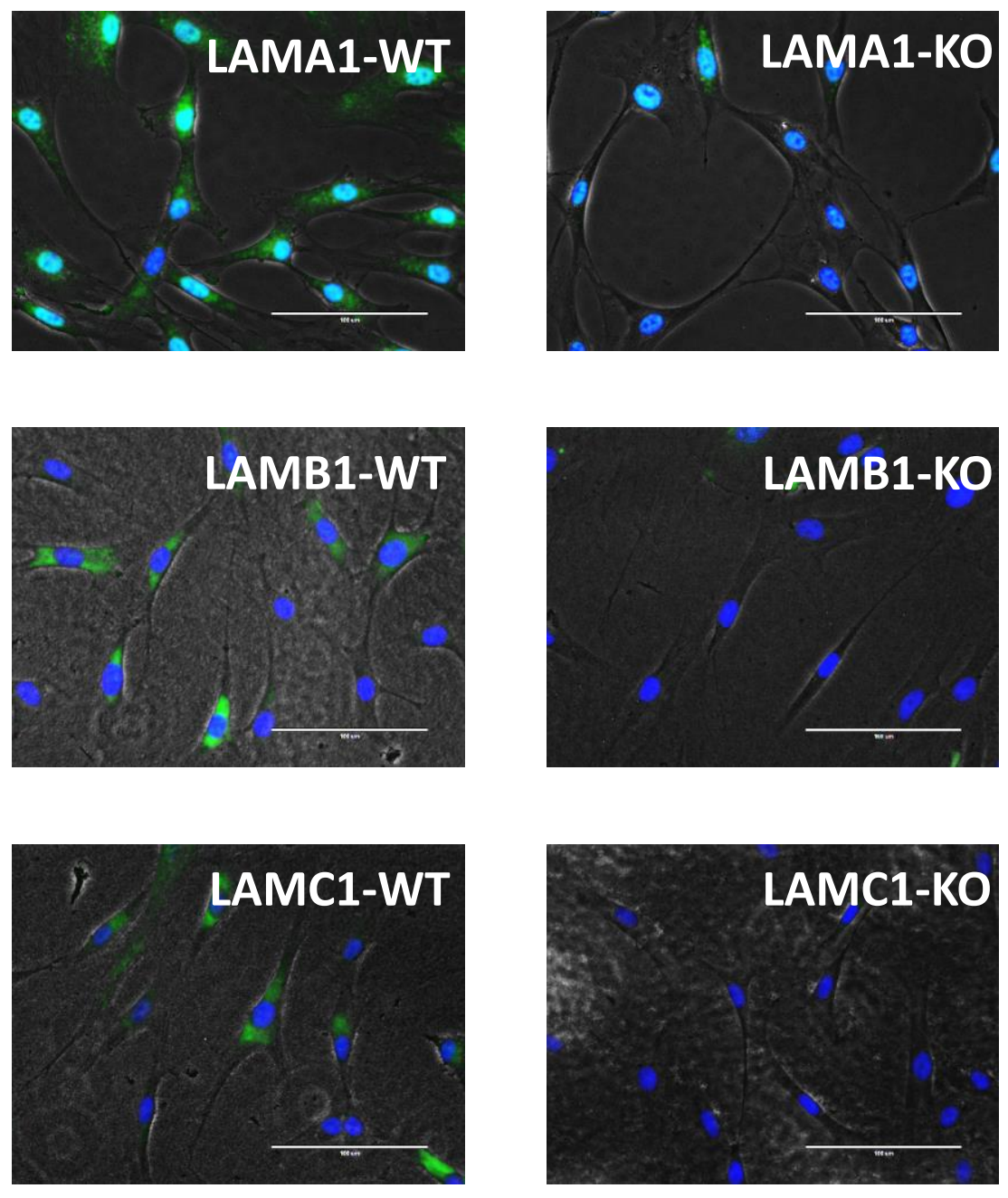

Figure 6.1: Immunofluorescence of wildtype (WT) and laminin knockout (KO) NPCs. Laminin wildtype (left column), and laminin gene knockouts (right column) for LAMA1 ( $\alpha-1$ subunit), LAMAB1 ( $\beta-1$ subunit), and LAMC1 ( $\gamma$-1 subunit). Green = respective Laminin subunit stain; Blue = DAPI-stained nuclei . 


\section{Figure 6.2}
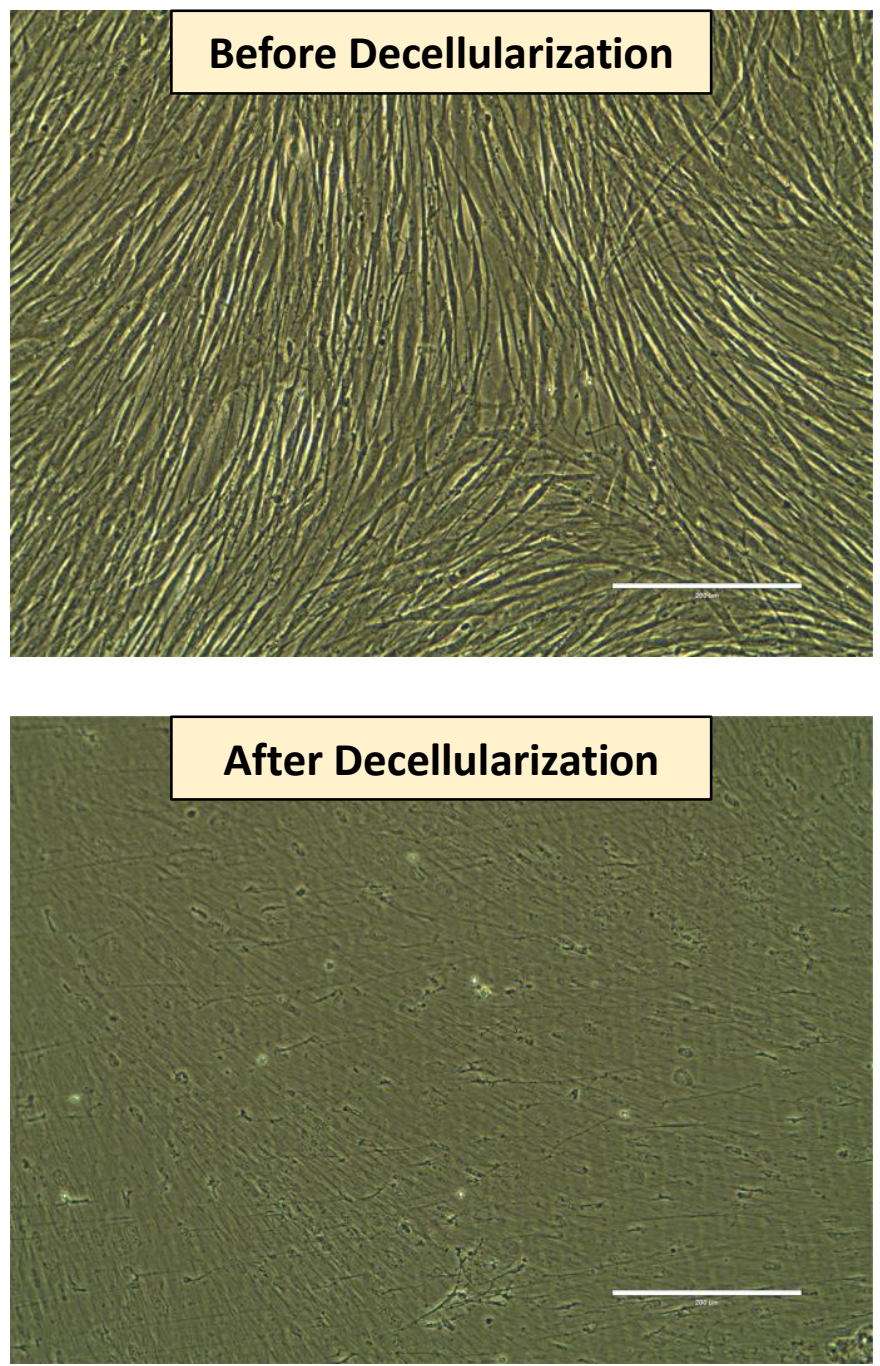

Figure 6.2: Preparation of dECM. Decellularized extracellular matrix can be produced by seeding a population of adherent cells on coated gelatin flasks, stimulating with ascorbic acid and growing to confluence (top). After decellularization, matrix components are left behind (bottom), offering an enhanced substrate for the expansion of new cells. 
Figure 6.3

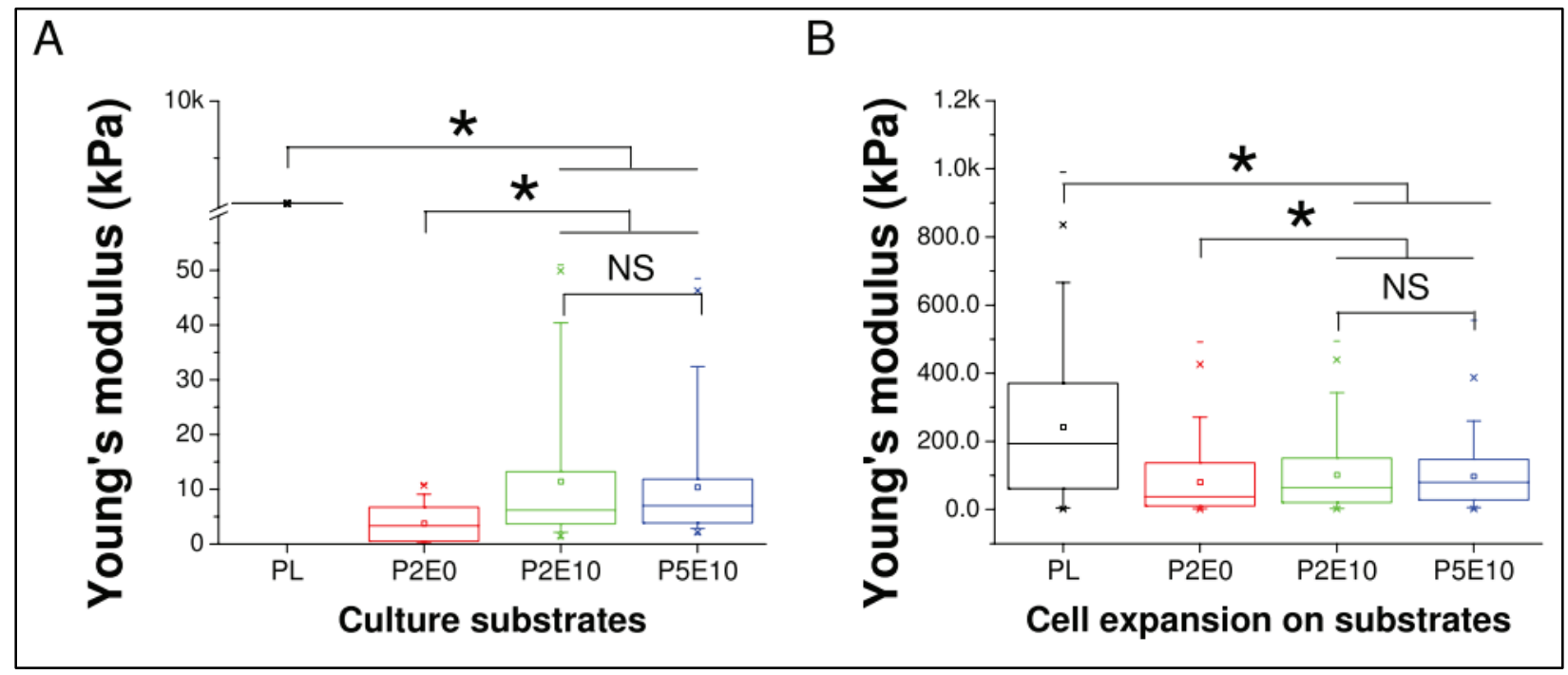

Figure 6.3: Biomechanical evaluation of both culture substrates and expanded cells in the duration regimens of AA treatment study. AFM was used to measure stiffness of each dECM group (PL, P2E0, P2E10, and P5E10) (average sample size $\mathrm{n}=274)(\mathrm{A})$ and corresponding IPSCs (average sample size $n=587$ ) grown on each above substrate (B). The stiffness of PL (A) is considered as infinite. ${ }^{*} \mathrm{p}<0.05$ indicates a statistically significant difference. NS: no statistically significant difference.

[22] Pizzute T, Zhang Y, He F, Pei M. Ascorbate-dependent impact on cell-derived matrix in modulation of stiffness and rejuvenation of infrapatellar fat derived stem cells toward chondrogenesis. Biomed Mater. 2016 Aug 10;11(4):045009. 


\section{References}

[1] Pei M. Environmental preconditioning rejuvenates adult stem cells' proliferation and chondrogenic potential. Biomaterials. 2016 Nov 25;117:10-23.

[2] Chen SQ, Lin JP, Zheng QK, Chen SJ, Li M, Lin XZ, Wang SZ. Protective effects of paeoniflorin against FasL-induced apoptosis of intervertebral disc annulus fibrosus cells via Fas-FasL signalling pathway. Exp Ther Med. 2015 Dec;10(6):2351 2355.

Ma CJ, Liu X, Che L, Liu ZH, Samartzis D, Wang HQ. Stem Cell Therapies for Intervertebral Disc Degeneration: Immune Privilege Reinforcement by Fas/FasL Regulating Machinery. Curr Stem Cell Res Ther. 2015;10(4):285-95.

Stich S, Stolk M, Girod PP, Thomé C, Sittinger M, Ringe J, Seifert M, Hegewald AA. Regenerative and immunogenic characteristics of cultured nucleus pulposus cells from human cervical intervertebral discs. PLoS One. 2015 May 19;10(5):e0126954.

Yuan T, Luo H, Tan J, Fan H, Zhang X. The effect of stress and tissue fluid microenvironment on allogeneic chondrocytes in vivo and the immunological properties of engineered cartilage. Biomaterials. 2011 Sep;32(26):6017-24.

[6] Yang J, Chen X, Yuan T, Yang X, Fan Y, Zhang X. Regulation of the secretion of immunoregulatory factors of mesenchymal stem cells (MSCs) by collagen-based scaffolds during chondrogenesis. Mater Sci Eng C Mater Biol Appl. 2017 Jan 1;70(Pt 2):983-991.

Zhang Y, Pizzute T, Li J, He F, Pei M. sb203580 preconditioning recharges matrix-expanded human adult stem cells for chondrogenesis in an inflammatory environment - A feasible approach for autologous stem cell based osteoarthritic cartilage repair. Biomaterials. 2015 Sep;64:88-97 

vitro microenvironment facilitates synovium-derived stem cell-based nucleus pulposus tissue regeneration. Spine (Phila Pa 1976). 2012 Aug 15;37(18):1538-47.

[9]

He F, Pei M. Rejuvenation of nucleus pulposus cells using extracellular matrix deposited by synovium-derived stem cells. Spine (Phila Pa 1976). 2012 Mar 15;37(6):459-69.

[10] He F, Chen X, Pei M. Reconstruction of an in vitro tissue-specific microenvironment to rejuvenate synovium-derived stem cells for cartilage tissue engineering. Tissue Eng Part A. 2009 Dec;15(12):3809-21.

[11] Bridgen DT, Gilchrist CL, Richardson WJ, Isaacs RE, Brown CR, Yang KL, Chen J, Setton LA. Integrin-mediated interactions with extracellular matrix proteins for nucleus pulposus cells of the human intervertebral disc. J Orthop Res. 2013 Oct;31(10):1661-7.

[12] Gilchrist CL, Francisco AT, Plopper GE, Chen J, Setton LA. Nucleus pulposus cell-matrix interactions with laminins. Eur Cell Mater. 2011 Jun 20;21:523-32.

Chen J, Jing L, Gilchrist CL, Richardson WJ, Fitch RD, Setton LA. Expression of laminin isoforms, receptors, and binding proteins unique to nucleus pulposus cells of immature intervertebral disc. Connect Tissue Res. 2009;50(5):294-306.

[14] Pizzute T, Zhang Y, He F, Pei M. Ascorbate-dependent impact on cell-derived matrix in modulation of stiffness and rejuvenation of infrapatellar fat derived stem cells toward chondrogenesis. Biomed Mater. 2016 Aug 10;11(4):045009.

[15] Tyndall A. Mesenchymal stem cell treatments in rheumatology: a glass half full? Nat Rev Rheumatol. 2014 Feb;10(2):117-24. 
[16] Prockop DJ, Prockop SE, Bertoncello I. Are clinical trials with mesenchymal stem/progenitor cells too far ahead of the science? Lessons from experimental hematology. Stem Cells. 2014 Dec;32(12):3055-61.

[17] Azamar-Llamas D, Hernández-Molina G, Ramos-Ávalos B, Furuzawa-Carballeda J. Adipokine Contribution to the Pathogenesis of Osteoarthritis. Mediators Inflamm. 2017;2017:5468023.

[18] Pizzute T, Lynch K, Pei M. Impact of tissue-specific stem cells on lineage specific differentiation: a focus on musculoskeletal system. Stem Cell Rev Rep 2015;11:119-32. 


\section{Permissions to Reproduce Copyrighted Materials}

Copyright
Clearance
Center

Cell \&Tissue
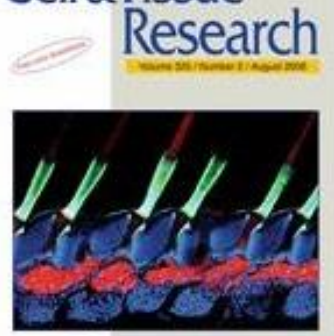

Limet

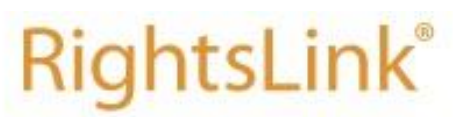

Title: impact on cartilage regeneration

Author:

$$
\text { Ying Zhang }
$$

Publication: Cell and Tissue Research

Publisher: Springer

Date:

Jan 1,2014

Copyright (5) 2014, Springer-Verlag Berlin Heidelberg

Quick Price Estimate

I would like to...?

Portion 2

Are you the author of this Springer

article?

\section{Select your currency}

Quick Price

\section{use in a thesis/dissertation}

make a selection

make a selection $\mathbf{v}$

USD - \$ $\quad$

Click Quick Price
LOGIN

If you're a copyright.com user, you can login to RightsLink using your copyright.com credentials. Already a RightsLink user or want to learn more?

The material can only be used for the purpose of defending your thesis limited to university-use only. If the thesis is going to be published, permission needs to be re-obtained (selecting "book/textbook" as the type of use). 
Copyright

Title:

Fibroblast Growth Factor Ligand Dependent Proliferation and Chondrogenic Differentiation of Synovium-Derived Stem Cells and Concomitant Adaptation of Wnt/Mitogen-Activated Protein Kinase Signals

Author: Tyler Pizzute, Jingting Li, Ying Zhang, et al

Publication: Tissue Engineering Part A

Publisher: Mary Ann Liebert, Inc.

Date: Aug 1, 2016

Copyright (c) 2016, Mary Ann Liebert, Inc.

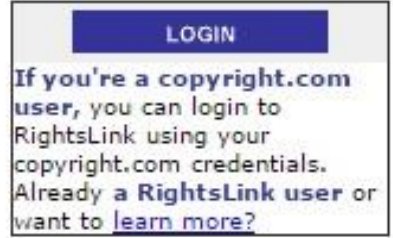
want to learn more?

\section{Quick Price Estimate}

Mary Ann Liebert, Inc. publishers does not require authors of the content being used to obtain a license for their personal reuse of full article, charts/graphs/tables or text excerpt.

I would like to...?

I am a/an... (1)

I would like to use... (3)

My format is... ?

I will be translating... ?

Distribution quantity ?

My currency is...

Quick Price

$$
\begin{aligned}
& \text { reuse in a journal/magazin } \\
& \text { author of the requested cont } \\
& \text { full article } \\
& \text { make a selection }
\end{aligned}
$$$$
\text { author of the requested content }
$$

make a selection $\mathbf{v}$

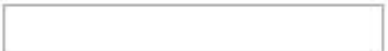

USD - \$

Click Quick Price
- No content delivery. This service provides permission for reuse only. If you do not have a copy of the article you are using, you may copy and paste the content and reuse according to the terms of your agreement.

To request a copy please contact reprints@liebertpub.com 
Copyright

Clearance Center

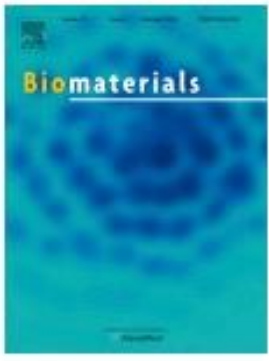

Title:

sb203580 preconditioning recharges matrix-expanded human adult stem cells for chondrogenesis in an inflammatory environment - A feasible approach for autologous stem cell based osteoarthritic

LOGIN cartilage repair

Author: $\quad$ Ying Zhang,Tyler Pizzute,Jingting Li,Fan He,Ming Pei

Publication: Biomaterials

Publisher: Elsevier

Date: September 2015

Copyright (s) 2015 Elsevier Ltd. All rights reserved.

Quick Price Estimate

This reuse request is free of charge although you are required to obtain a license through Rightslink and comply with the license terms and conditions. You will not be charged for this order. To complete this transaction, click the Continue button below.

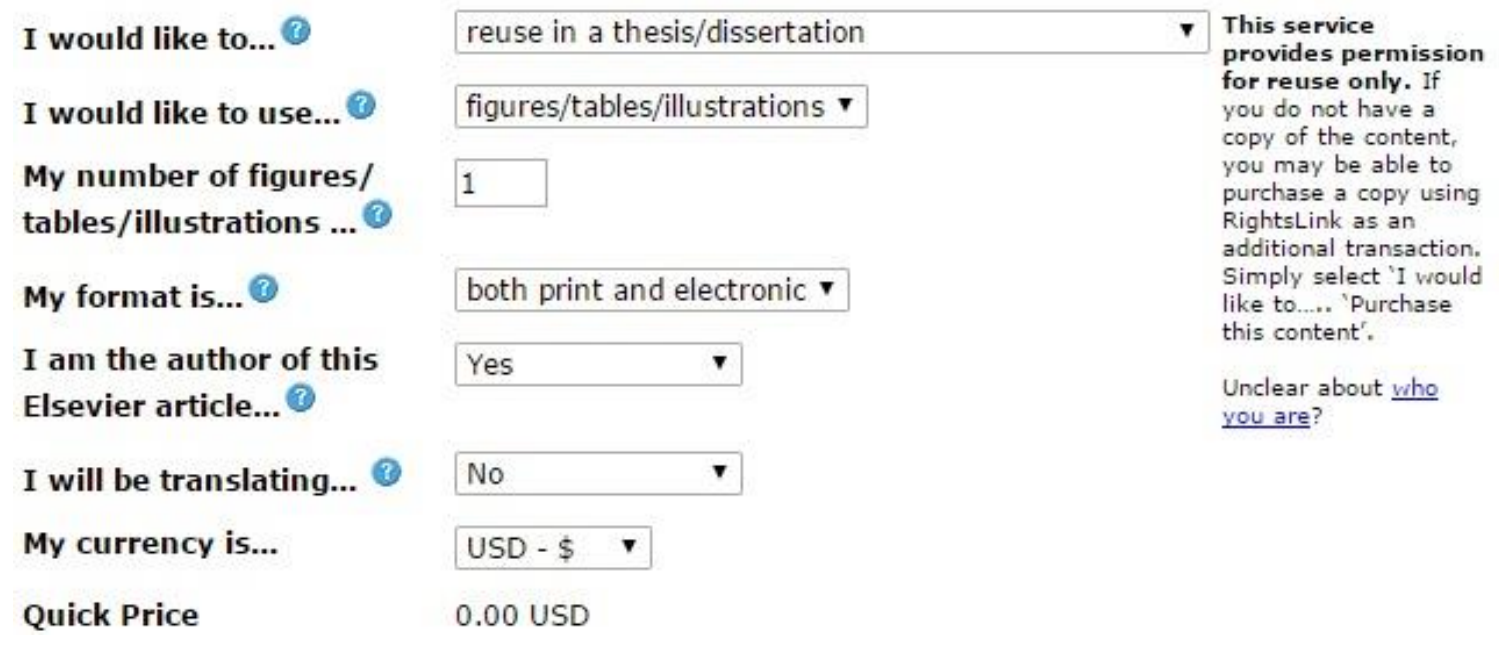


Biomedical Materials
ISSN:
$1748-605 x$
Language: English
Publication year(s): 2006 - present
Country of publication: United Kingdom of Great
Britain and Northern e-Journal
IOP Publishing
Publisher:
IOP PUBLISHING, LTD
Rightsholder:

Republish or display content

Permission type selected:

Type of use selected: Thesis/Dissertation

\& Select different permission

Total Price: $\$ \mathbf{0 . 0 0}$

Terms and conditions apply to this permission type View details

No royalties will be charged for this reuse request although you are required to obtain a license and comply with the license terms and conditions. To obtain the license, click the Continue button below.

Enter the following details to determine a price:

Describe who will republish the content (person or entity)... (3)

I would like to use... 0

Number of charts/graphs/tables/figures (3)

I want rights for... 0

My format is... ?

Academic institution $\quad \mathbf{v}$

chart/graph/table/figure

7

Main product

Print $\downarrow$ Electronic

Duration of use... (0)

Creation of copies for the disabled...?

With minor editing privileges... 3

For distribution to...?

In the following language(s)...?

With incidental promotional use... 0

The lifetime unit quantity of new product... ?

Promo code... (optional)?

Total Price: $\$ \mathbf{0 . 0 0}$ 


\section{Copyright Clearance Center}

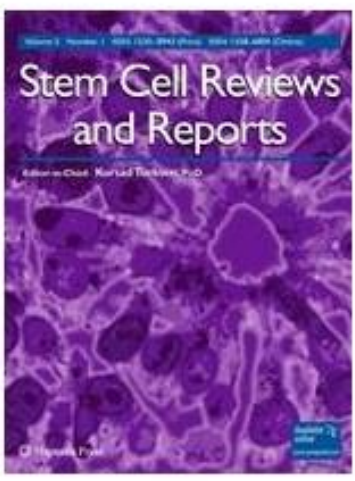

Title:

Author:

Impact of Tissue-Specific Stem Cells on Lineage-Specific Differentiation: A Focus on the Musculoskeletal System

Publication: Stem Cell Reviews

Publisher: Springer

Date: Jan 1, 2014

Copyright (9) 2014, Springer Science+Business Media New York

\begin{tabular}{|l|}
\multicolumn{1}{|c|}{ LOGIN } \\
If you're a copyright.com \\
user, you can login to \\
RightsLink using your \\
copyright.com credentials. \\
Already a RightsLink user or \\
want to learn more?
\end{tabular}

want to learn more?

Quick Price Estimate

I would like to... (3)

Portion 3

Number of copies $?$

Are you the author of this Springer article?

Select your currency

Quick Price use in a thesis/dissertation

Full text

make a selection $\mathbf{v}$ v No content delivery. This service provides permission for reuse only. Once licensed, you may use the content according to the terms of your license.

Price quoted is an estimate based on this request for this title only. Final price will depend on the total amount of requested Springer material.

USD - \$

Click Quick Price

The material can only be used for the purpose of defending your thesis limited to university-use only. If the thesis is going to be published, permission needs to be re-obtained (selecting "book/textbook" as the type of use). 

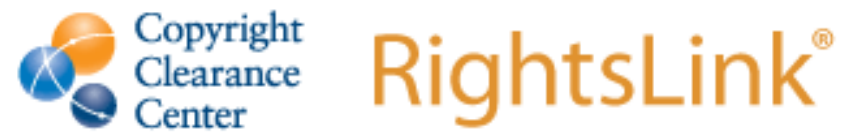

Creative Commons Attribution License (CC BY)

This article is available under the terms of the Creative Commons Attribution License (CC BY).

You may copy and distribute the article, create extracts, abstracts and new works from the article, alter and revise the article, text or data mine the article and otherwise reuse the article commercially (including reuse and/or resale of the article) without permission from Elsevier. You must give appropriate credit to the original work, together with a link to the formal publication through the relevant DOI and a link to the Creative Commons user license above. You must indicate if any changes are made but not in any way that suggests the licensor endorses you or your use of the work.

Permission is not required for this type of reuse.

\section{CLOSE WINDOW}

Copyright (9) 2017 Copyright Clearance Center, Inc. All Rights Reserved.

Comments? We would like to hear from you. E-mail us at customercare@copyright.com 


\section{CURRICULUM VITAE}

TYLER T. PIZZUTE

\section{EDUCATION \& TRAINING}

2012-2017 Ph.D. in Exercise Physiology, Stem Cell and Tissue Engineering Department of Orthopaedics, West Virginia University, Morgantown, WV

2013-2017 Graduate Research Assistant

Department of Orthopaedics, West Virginia University, Morgantown, WV

2008-2012 B.S. in Biology; Chemistry minor

Walsh University, North Canton, $\mathrm{OH}$

\section{RESEARCH INTERNSHIPS}

$2011 \quad$ Undergraduate Research Intern (Biomaterials)

Syracuse Biomaterials Institute (SBI), Syracuse, NY

2010 Undergraduate Research Intern (Polymer Engineering)

University of Akron, Goodyear Polymer Center, Akron, $\mathrm{OH}$

2009-2012 Undergraduate Research Student (Chemistry)

Department of Chemistry, Walsh University, North Canton, $\mathrm{OH}$ 


\section{HONORS \& AWARDS}

2016 Featured Abstract in Atlas of Science (Feb. 2016): "Is a tissue-engineering approach effective for the treatment of osteoporotic bone defects?"

http://atlasofscience.org/is-a-tissue-engineering-approach-effective-for-the$\underline{\text { treatment-of-osteoporotic-bone-defects/ }}$

2015 Featured Abstract in Atlas of Science (Dec. 2015): "Recharged autologous stem cells to combat cartilage defects in osteoarthritic patients"

http://atlasofscience.org/recharged-autologous-stem-cells/

2008-2012 Vanasse Scholarship Recipient

Walsh University, North Canton, $\mathrm{OH}$

2008-2012 LifeScholars Recipient

Walsh University, North Canton, $\mathrm{OH}$

\section{PROFESSIONAL ASSOCIATIONS}

2016-Present Member - American Association for the Advancement of Science (AAAS)

2016-Present Member - Consulting Club at The Texas Medical Center

2011-Present Member - Sigma Zeta National Science \& Mathematics Honor Society

Walsh University Chapter, North Canton, $\mathrm{OH}$ 


\section{TEACHING \& LEADERSHIP}

2016-2017 Writer- West Virginia Health Ambassadorship Program

West Virginia University, Morgantown, WV

2013-2017 Project Supervisor/Leader - Stem Cell \& Tissue Engineering Laboratory

West Virginia University, Morgantown, WV

2015-2017 Student Ambassador - Interdisciplinary Biomedical Sciences Graduate Program

West Virginia University, Morgantown, WV

2012 Assistant Lacrosse Coach - Boys’ High School (Grade 9-12)

Lake High School, Uniontown, $\mathrm{OH}$

2008-2010 Board Member - Health Technologies Preparatory Program

Lake High School, Uniontown, $\mathrm{OH}$

\section{PEER-REVIEWED PUBLICATIONS}

1. Pizzute T, Li JT, Zhang Y, Pei M. FGF ligand dependent proliferation and multidifferentiation of synovium-derived stem cells and concomitant adaptation of Wnt/MAPK signals during chondrogenesis. Tissue Eng Part A 2016;22(15-16):1036-46. doi: 10.1089/ten.TEA.2016.0102. PMID: 27411850 (IF: IF: 4.448)

2. Pizzute T, Zhang Y, He F, Pei M. Ascorbate-dependent impact on cell-derived matrix in modulation of stiffness and rejuvenation of infrapatellar fat derived stem cells toward chondrogenesis. Biomed Mater 2016;11:045009. doi: 10.1088/1748-6041/11/4/045009. PMID: 27508528 (IF: 3.697) (feature article in Mesenchymal Cell News 8.32 August 16, 2016) 
3. Pizzute T, Lynch K, Pei M. Impact of tissue-specific stem cells on lineage specific differentiation: a focus on musculoskeletal system. Stem Cell Rev Rep 2015;11:119-32. doi: 10.1007/s12015-014-9546-8. PMID: 25113801 PMCID: PMC4326629 (IF: 4.523)

4. Zhang Y, Pizzute T, Li JT, He F, Pei M. sb203580 preconditioning recharges matrixexpanded human adult stem cells for chondrogenesis in an inflammatory environment - a feasible approach for autologous stem cell based osteoarthritic cartilage repair. Biomaterials 2015;64:88-97. PMID: 26122165. doi: 10.1016/j.biomaterials.2015.06.038. (IF: 8.557) (highlighted in F1000Prime) (highlighted in Atlas of Science)

5. Zhang Y, Pizzute T, Pei M. A review of crosstalk between MAPK and Wnt signals and its impact on cartilage regeneration. Cell Tissue Res 2014;358:633-49. PMID: 25312291. PMCID: PMC4234693 (IF: 3.677)

6. Zhang Y, Pizzute T, Pei M. Anti-inflammatory strategies in cartilage repair. Tissue Eng Part B 2014;20:65568. PMID: 24846478 (IF: 4.64) (feature article in Mesenchymal Cell News 5.20 May 29, 2014)

\section{MANUSCRIPTS IN PREPARATION OR UNDER REVIEW}

7. Pizzute T, Emery S, Zhang Y, Waris S, Pei M. Preconditioning strategies unequally impact regeneration of nucleus pulposus cells from human herniated discs: a proof of principle study. (under review)

8. Pizzute T, Pei M. Noncanonical WNT signals promote the early redifferentiation of human nucleus pulposus cells. (in preparation)

9. Pizzute T, Werner Z, Pei M. Preconditioning strategies for nucleus pulposus rejuvenation and impacts on cell immunity. (in preparation)

10. Pizzute T, Pei M. Preconditioning of nucleus pulposus cells and impacts on redifferentiation and immunity. (in preparation) 


\section{PUBLISHED ABSTRACTS}

1. Pizzute T, Zhang Y, Zhang XB, Pei M. Wnt signals promote redifferentiation of human nucleus pulposus cells harvested from herniated discs. 63rd Annual Meeting of the Orthopaedic Research Society. San Diego Convention Center, San Diego, California (2017)

2. Pizzute T, Pei M. Potential use of nonautologous cell matrix for rejuvenation of human nucleus pulposus cells from herniated discs. 63rd Annual Meeting of the Orthopaedic Research Society. San Diego Convention Center, San Diego, California (2017)

3. Pizzute T, Waris SA, Zhang Y, Emery SE, Pei M. Preconditioning strategies unequally impact regeneration of nucleus pulposus cells from human herniated discs. International Cartilage Research Society (ICRS) 2016. Sorento-Naples, Italy, Sept 24-27, 2016 (Oral presentation)

4. Pei M, Li J, Pizzute T, Davis M, Hansen K, van Wijnen AJ. Characterization of matrix microenvironment for stem cell based chondrogenesis. International Cartilage Research Society (ICRS) 2016. Sorento-Naples, Italy, Sept 24-27, 2016 (Oral presentation)

5. Pei M, Zhang Y, Pizzute T, Dong CB, Dinu C. Ascorbate-dependent impact on cellderived matrix in modulation of stiffness and rejuvenation of infrapatellar derived stem cells toward chondrogenesis. 62th Annual Meeting of the Orthopaedic Research Society. Disney's Coronado Springs Resort, Orlando, Florida (2016)

6. Pizzute T, Waris S, Zhang Y, Pei M. Preconditioning strategies impact redifferentiation of nucleus pulposus from human herniated discs. 2016 E. J. Van Liere Convocation and Research Day, WVU, March 6.

7. Pizzute T, Pei M. Is a tissue-engineering approach effective for the treatment of osteoporotic bone defects. Atlas of Science, Feb 19, 2016. "http://atlasofscience.org/is-atissue-engineering-approacheffective-for-the-treatment-of-osteoporotic-bone-defects" 
8. Pei M, Li JT, Pizzute T, Dong CB, Hansen K, Dinu C. Decode a niche-like microenvironment to facilitate cell-based disc regeneration. ORS PSRS 3rd International Philadelphia Spine Research Symposium. Nov 9-12, 2015.

9. Pizzute T, Pei M. Recharged autologous stem cells to combat cartilage defects in osteoarthritic patients. Atlas of Science, Dec 30, 2015. "http://atlasofscience.org/recharged-autologous-stem-cells/"

10. Waris SA, Pizzute T, Zhang Y, Pei M. Preconditioning strategies impact regeneration of nucleus pulposus from human herniated discs. SURE (Summer Undergraduate Research Experience) Research Symposium, July 23, 2015

11. Pizzute T, Li JT, Zhang Y, Ohliger J, Pei M. The effect of fibroblast growth factor supplementation on multilineage stem cell differentiation. 2015 E. J. Van Liere Convocation and Research Day, WVU, Feb 28.

12. Li JT, Zhang Y, Pizzute T, Pei M. Contribution of FGF2 versus FGF10 to synoviumderived stem cell expansion and tri-lineage differentiation and potential mechanisms. 60th Annual Meeting of the Orthopaedic Research Society. Hyatt Regency New Orleans, New Orleans, Louisiana (2014)

13. Ohliger J, Li JT, Pizzute T, Pei M. The different influences of FGF-2 and FGF-10 on stem cell multidifferentiation. 2014 E. J. Van Liere Convocation and Research Day, WVU, Feb 28. (Second Place in Oral presentation) 